\title{
Distributed Multi-Agent Consensus under Constraints
}

\section{QIU ZHIRONG}

School of Electrical \& Electronic Engineering

A thesis submitted to the Nanyang Technological University

in partial fulfillment of the requirements for the degree of

Doctor of Philosophy 


\section{Statement of Originality}

I hereby certify that the work embodied in this thesis is the result of original research and has not been submitted for a higher degree to any other University or Institution. 


\section{Acknowledgements}

This thesis concludes my research work over the past four years of $\mathrm{PhD}$ studies. Were it not for the great help and support from many people, I would have never survived this tough journey. Your kindness and benevolence shall be remembered and cherished for always.

First and foremost, I owe my deepest gratitude to my supervisor, Prof. Xie Lihua, for his unconditional support and professional guidance. I am specially grateful to his toleration of allowing me to explore different research topics, and I have learned a great deal from his meticulous scholarship.

I would also like to express my most sincere thankfulness to Prof. Hong Yiguang, for his penetrating insight and persistent encouragement. He guides me as my co-supervisor, and I definitely appreciate his frankness in lecturing me and exposing my naivety.

I am also willing to thank Dr Liu Shuai who introduced me into the area of distributed optimization. Special thanks go out to Dr You Keyou for the golden opportunity he brought to me to study abroad, Danny Prince and Bryce Pan for their fraternity and camaraderie, as well as the other colleagues (especially Alex and Mary) in the Internet of Things Lab for their care and company.

Finally, I am mostly indebted to my family, were it not for whom I would have long before ceased the struggle and even my own existence. This thesis is dedicated to them, as a trivial tribute for their years-long austerity and abnegation. 



\section{Abstract}

As a basic problem in the cooperative control of multi-agent systems (MASs), distributed multiagent consensus aims to achieve an agreement of certain interested variables among a group of agents by exchanging information between neighbors. It has found a variety of engineering applications in different areas, e.g. coordination, rendezvous, flocking and formation control. To implement consensus in practice, we need to consider different kinds of constraints. On the one hand, practical MASs bear their own physical limits, such as limited communication bandwidth and kinematic constraints; on the other hand, the final consensus value needs to satisfy further requirements in some specific tasks. This thesis is dedicated to the study of two specific consensus problems under constraints: quantized consensus under data rate constraint, and optimal consensus under set or kinematic constraints.

The first part of the thesis considers the quantized consensus problem under data rate constraint. In a multi-agent network, any communication channel has limited capacity and only finite bits of errorfree information can be transmitted per unit time. As a result, the consensus protocol is designed in the presence of quantized signals and limited data rate. Although the result on using finite bits of data exchange to achieve quantized consensus for MASs with some general linear dynamics has been established, it is still an open problem about how many bits of data suffice to achieve consensus for systems with high-order dynamics and a partially measured state, and it is not clear what determines the sufficient data rate for consensus In this work, we focus on the quantized consensus for two kinds of critical high-order dynamics: $n$-th order integrators $(n \geq 3)$, and $2 m$-th order oscillators $(m \geq 1)$ with $m$ identical pairs of conjugate poles on the unit circle. Besides, in either case only the first state variable is measurable. We design an observer-based dynamic encoding-decoding scheme to estimate the unmeasurable state variables, and devise a control protocol based on the encoder/decoder outputs. By employing perturbation techniques, we are able to explicitly design the control gains and provide a sufficient data rate for quantized consensus. Under a fixed and connected network, for MASs with integrator dynamics it only needs $n$ bits of information exchange to achieve exponentially fast consensus, while for oscillator dynamics the number of required bits is an integer between $m$ and $2 m$, which is dependent on the location of poles, or the oscillation frequency. These results not only achieve the lowest data rate among existing works, but also indicate that the suffcient data rate is independent of the network topology, and closely related with the system structure. 
The second part of the thesis considers the optimal consensus problem under set or kinematic constraints. The optimal consensus aims to achieve an agreement of state that minimizes the aggregate cost, which is the sum of individual costs assigned to each agent. A typical example is the shortestdistance rendezvous, which may be further required to be achieved within a given constraint set. Continuous-time MASs with single integrator dynamics are first studied. To achieve the constrained optimum, we combine three different terms into the control protocol: local averaging, local projection, and local subgradient descent with a decaying gain $\alpha(t)$. Under a balanced network with uniformly joint spanning trees, we employ non-smooth analysis to show that the convergence to the constrained optimum set can be guaranteed if $\alpha(t)$ is non-integrable. Provided that the aggregate cost is strongly convex, we further analyze the convergence rate for different types of $\alpha(t)$. In a similar vein, we establish corresponding results for discrete-time systems, and further discuss the necessary conditions and the issue of communication delay when the balanced network is fixed.

We also explore the optimal consensus under kinematic constraints, specifically for MASs with hererogeneous EL dynamics under bounded input and velocity. Assuming an exact knowledge of nonlinearity and a priori estimate of the optimum, we show that an exponentially fast convergence is guaranteed while satisfying the bounded kinematic constraints, if the fixed and undirected topology is connected. 


\section{Symbols and Acronyms}

$\begin{array}{ll}\text { Operators and Vectors } & \\ A^{\prime} & \text { The transpose of } A \\ A^{-1} & \text { The inverse of } A \\ A(i, j) & \text { The }(i, j) \text { entry of } A \\ A(i, \cdot) & \text { The } i \text {-th row of } A \\ A(\cdot, j) & \text { The } j \text {-th column of } A \\ \operatorname{det}(A) & \text { The determinant of } A \\ \operatorname{diag}\left\{M_{1}, \ldots, M_{n}\right\} & \text { The block diagonal matrix with the diagonal blocks } \\ & \text { given by } M_{1}, \ldots, M_{n} \\ \operatorname{col}\left\{x_{1}, \ldots, x_{n}\right\} & \text { The column vector }\left[x_{1}^{\prime}, \ldots, x_{n}^{\prime}\right]^{\prime} \\ |\cdot| & \text { Absolute value } \\ |\cdot| \mathcal{X} & \text { Distance to set } \mathcal{X} \\ \|\cdot\| & \text { Euclidean norm } \\ \|\cdot\|_{\infty} & \text { Infinity norm } \\ \|\cdot\|_{1} & 1 \text {-norm } \\ \otimes & \text { The Kronecker product } \\ \langle\cdot, \cdot\rangle & \text { The standard inner product in Euclidean spaces } \\ \operatorname{Re}(\cdot) & \text { The real component of a complex number } \\ \operatorname{Im}(\cdot) & \text { The imaginary component of a complex number } \\ I_{n} & \text { The } n \text {-dimensional identity matrix } \\ J_{\lambda, n} & \text { The } n \text {-dimensional Jordan block with eigenvalue } \lambda \\ \mathbf{1}_{n} & \text { The } n \text {-dimensional column vector of ones } \\ C(n, k) & \text { The number of } k \text {-combinations from a given set of } n \\ J & \text { elements } \\ \lfloor\cdot\rfloor & \text { The unit imaginary number } \\ \lceil\cdot\rceil & \text { The floor function function } \\ & \\ & \end{array}$


$O(\cdot)$

$o(\cdot)$

$\nabla$

Sets

$\mathbb{R}$

$\mathbb{R}_{\geq 0}$

$\mathbb{N}^{+}$

\section{Acronyms}

MAS

EL

$\mathrm{AC}$

iff

a.e.

a.c.
An infinitesimal of equal or higher order, e.g. $f(\varepsilon)=$ $O(g(\varepsilon))$ if and only if $\limsup _{\varepsilon \rightarrow 0} \frac{|f(\varepsilon)|}{|g(\varepsilon)|}<\infty$

An infinitesimal of higher order, e.g. $f(\varepsilon)=o(g(\varepsilon))$ if and only if $\limsup _{\varepsilon \rightarrow 0} \frac{|f(\varepsilon)|}{|g(\varepsilon)|}=0$

The vector differential operator

The set of real numbers

The set of nonnegative real numbers

The set of positive integers

multi-agent system

Euler-Lagrangian

Average Consensus

if and only if

almost everywhere

absolutely continuous 


\section{Contents}

\begin{tabular}{|ll}
\hline Acknowledgment & ii
\end{tabular}

\begin{tabular}{ll}
\hline Abstract & iii
\end{tabular}

\begin{tabular}{|l|l|}
\hline Symbols and Acronyms & v \\
\hline
\end{tabular}

\begin{tabular}{ll}
\hline Table of Contents & ix \\
\hline
\end{tabular}

\begin{tabular}{|c|c|}
\hline List of Figures & $\mathrm{x}$ \\
\hline
\end{tabular}

\begin{tabular}{lll}
\hline & Introduction & 1
\end{tabular}

1.1 Motivation and Objectives . . . . . . . . . . . . . . . . . . . . 1

1.2 Major Contributions and Outline . . . . . . . . . . . . . . . . . . . . . . . 3

\begin{tabular}{|lll}
\hline 2 & Literature Review & 7 \\
\hline
\end{tabular}

2.1 Quantized Consensus . . . . . . . . . . . . . . . . . . . . . . . . . 8

2.2 Optimal Consensus . . . . . . . . . . . . . . . . . . . . . . . . . . . 10

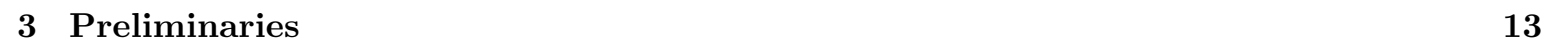

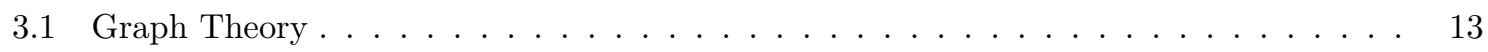

3.2 Stochastic Matrix Theory . . . . . . . . . . . . . . . . . . . . . . . . . . . 14

3.3 Convex Analysis . . . . . . . . . . . . . . . . . . . . . . . . . . . . . . 15

3.3 .1 Convex Sets . . . . . . . . . . . . . . . . . . . 15

3.3 .2 Convex Functions $\ldots \ldots \ldots \ldots \ldots$

$\begin{array}{llll}\text { Part } & \text { Quantized Consensus under Data Rate Constraint } & 17\end{array}$

4 Quantized Consensus of $n$-th Order Integrator Systems under Data Rate Constraint

4.1 Problem Formulation . . . . . . . . . . . . . . . . . . . . . . . . . . . . . . . . . . . 19

4.2 Quantized Consensus and Data Rate for $n$-th Order Integrator Dynamics . . . . . . . 20

$4.2 .1 \quad$ Observer-based Encoding-Decoding Scheme . . . . . . . . . . . . . . . . . . 20 
4.2 .2 Leaderless Consensus.$\ldots \ldots \ldots \ldots$

$4.2 .3 \quad$ Leader-following Consensus $\ldots \ldots \ldots \ldots$. . . . . . . . . . . . . . . . . . 32

4.3 Quantized Consensus and Data Rate for General Systems with Unity Eigenvalues . . . 34

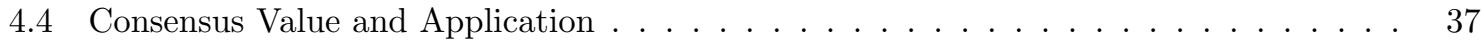

4.5 Numerical Examples . . . . . . . . . . . . . . . . . . . . . . 39

4.6 Conclusion $\ldots \ldots \ldots \ldots \ldots$

4.7 Proof of Main Lemmas . . . . . . . . . . . . . . . . . . . . . . . . . 41

5 Quantized Consensus of $2 m$-th Order Oscillator Systems under Data Rate Constraint $\quad 49$

5.1 Problem Formulation $\ldots \ldots \ldots \ldots \ldots \ldots$

5.2 Harmonic Oscillator Case $\ldots \ldots \ldots \ldots \ldots \ldots$. . . . . . . . . . . . . . . 51

$5.2 .1 \quad$ Encoding-decoding Scheme and Distributed Control Law $\ldots . . . . . . .551$

5.2 .2 Consensus Analysis and Data Rate . . . . . . . . . . . . . . . 53

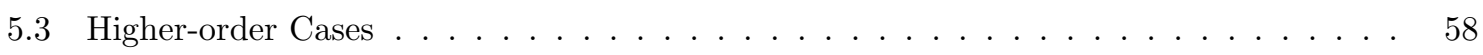

$5.3 .1 \quad$ Encoding-decoding Scheme and Distributed Control Law . . . . . . . . . 58

5.3 .2 Lemmas . . . . . . . . . . . . . . . . . . . . . . . . . 61

$5.3 .3 \quad$ Convergence Analysis and Data Rate . . . . . . . . . . . . . . . 63

5.4 Numerical Examples $\ldots \ldots \ldots \ldots \ldots$

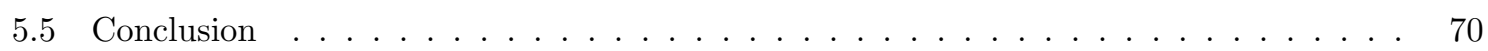

5.6 Proof of Main Lemmas $\ldots \ldots \ldots \ldots$

$\begin{array}{llll}\text { Part } & \text { Optimal Consensus under Set or Kinematic Constraints } & 81\end{array}$

6 Continuous-time Optimal Consensus under Set Constraint 82

6.1 Problem Formulation . . . . . . . . . . . . . . . . . . . . . . . . . . . . . . . . 82

6.2 Control Design and Convergence Result $\ldots \ldots \ldots \ldots \ldots$

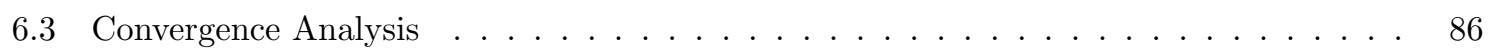

$6.3 .1 \quad$ Preliminaries on Differential Equations with Discontinuous Righthand Sides . . 87

6.3 .2 Globally Bounded Solution . . . . . . . . . . . . . . . . . . . 88

6.3 .3 Constraint Set Convergence . . . . . . . . . . . . . . . . . . 90

6.3 .4 Consensus . . . . . . . . . . . . . . . . . . . . . . . 91

6.3 .5 Optimum Set Convergence . . . . . . . . . . . . . . . . . 94

6.4 Convergence Rate Analysis . . . . . . . . . . . . . . . . . . . . . . . . . . . . . . 95

6.4 .1 Set Convergence Rate . . . . . . . . . . . . . . . . . 96

6.4 .2 Consensus Rate . . . . . . . . . . . . . . . . . . . . . . 96

6.4 .3 Optimum Set Convergence Rate . . . . . . . . . . . . . . . . . . . 97 
6.5 Numerical Examples $\ldots \ldots \ldots \ldots \ldots$

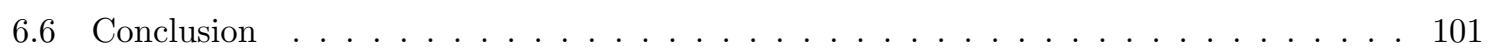

$\begin{array}{lll}7 & \text { Discrete-time Optimal Consensus under Set Constraint } & 103\end{array}$

7.1 Problem Formulation and Control Protocol . . . . . . . . . . . . . . . . . . . . . . 104

7.2 Convergence Analysis $\ldots \ldots \ldots \ldots \ldots \ldots \ldots \ldots$

7.3 Convergence Rate Analysis $\ldots \ldots \ldots \ldots$. . . . . . . . . . . 110

7.4 Further Discussion for a Fixed Network $\ldots \ldots \ldots \ldots$. . . . . . . . . . . . 116

$7.4 .1 \quad$ Necessary and Sufficient Conditions _ . . . . . . . . . . . . . . . . . . . 116

$7.4 .2 \quad$ Convergence under Fixed Time Delays $\ldots \ldots \ldots \ldots$

7.5 Numerical Examples $\ldots \ldots \ldots \ldots$. . . . . . . . . . . . . . . . . . 124

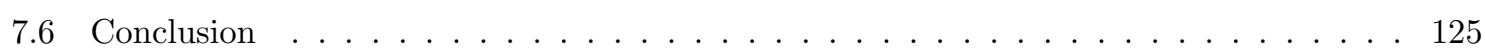

8 Optimal Consensus of Euler-Lagrangian Systems under Kinematic Constraints 127

8.1 Problem Formulation . . . . . . . . . . . . . . . . . . . . . . . . . . . . . . 128

8.2 Optimal Consensus of MAS with Double Integrator Dynamics and Kinematic Constraints 129

8.3 Optimal Consensus of MAS with EL Dynamics and Kinematic Constraints . . . . . 135

$8.4 \quad$ Numerical Examples $\ldots \ldots \ldots \ldots$. . . . . . . . . . . . . . . . . . . . 137

8.5 Conclusion . . . . . . . . . . . . . . . . . . . . . . . . . . . 140

$\begin{array}{lll}9 \text { Conclusion and Future Work } & 141\end{array}$

9.1 Conclusion . . . . . . . . . . . . . . . . . . . . . . . . . . . . . . . . . . . . 141

9.2 Future Work . . . . . . . . . . . . . . . . . . . . . . . . . . 142

\begin{tabular}{lr}
\hline Author's Selected Publications & 145
\end{tabular}

\begin{tabular}{lr}
\hline Bibliography & 147
\end{tabular} 


\section{List of Figures}

1.1 Workflow in quantized consensus. . . . . . . . . . . . . . . . . . . 2

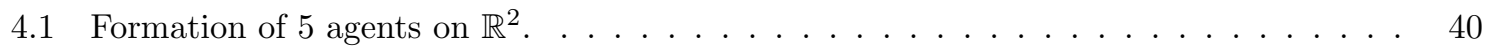

4.2 Velocity consensus. . . . . . . . . . . . . . . . . . . . . . . . . . 40

4.3 Acceleration consensus. . . . . . . . . . . . . . . . . . . . . . . 40

4.4 Newton diagram. . . . . . . . . . . . . . . . . . . . . . . . . . . . . . . . . 42

$5.1 \quad$ Convergence of maximum disagreements $\delta_{\text {jmax }}(t): \mathrm{m}=1 . \ldots \ldots \ldots \ldots \ldots$

5.2 Convergence of maximum disagreements $\delta_{\text {jmax }}(t): \mathrm{m}=2 . \ldots \ldots \ldots \ldots$

5.3 Newton diagram. . . . . . . . . . . . . . . . . . . . . . . . 71

6.1 Communication graphs. $\ldots \ldots \ldots \ldots \ldots$

6.2 Continuous-time optimal consensus without set constraint. . . . . . . . . . . . . . 101

6.3 Convergence of $x, y$ coordinates without set constraint. . . . . . . . . . . . . . . 101

6.4 Continuous-time optimal consensus with $\mathcal{X}=\{x:\|x\| \leq 1\} . \ldots \ldots \ldots \ldots \ldots$. . 102

$6.5 \quad$ Convergence of $x, y$ coordinates with $\mathcal{X}=\{x:\|x\| \leq 1\} \ldots \ldots \ldots \ldots \ldots \ldots$

$7.1 \quad$ Construction of virtual nodes. . . . . . . . . . . . . . . . . . . . . . . . . . . . . . 120

7.2 Discrete-time optimal consensus with set constraint. . . . . . . . . . . . . . 125

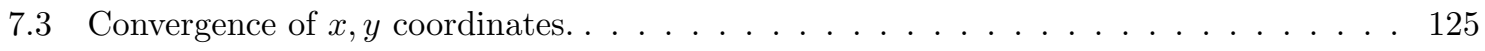

7.4 Communication graph with fixed time delays. . . . . . . . . . . . . . . . 126

7.5 Plane trajectories of 4 agents with fixed time delays. . . . . . . . . . . . . 126

7.6 Convergence of $x, y$ coordinates with fixed time delays. . . . . . . . . . 126

8.1 Optimal consensus of positions with small gains. . . . . . . . . . . . . 138

8.2 Variation of velocities with small gains. $\ldots \ldots \ldots \ldots \ldots \ldots \ldots$

8.3 Variation of accelerations with small gains. $\ldots \ldots \ldots \ldots \ldots \ldots$

8.4 Optimal consensus of positions with larger gains. . . . . . . . . . . . . . . . . 139

8.5 Variation of velocities with larger gains $\ldots \ldots \ldots \ldots \ldots \ldots \ldots$

8.6 Variation of accelerations with larger gains. . . . . . . . . . . . . . . . 139 


\section{Chapter 1}

\section{Introduction}

\subsection{Motivation and Objectives}

A multi-agent system (MAS) consists of a group of autonomous decision-making agents which possess sensing, computation and communication capabilities and aim to achieve a common goal through cooperation. In engineering applications, typical MASs include robot fleets and sensor networks, which are promising to play a more and more critical role in carrying out collective tasks in both civil and military areas, such as search and rescue, surveying, and disaster relief. Over the recent decade, considerable research effort has been devoted to the distributed control of MASs, and among them distributed consensus has attracted the most attention as a basic problem.

Briefly speaking, distributed consensus aims to reach an agreement of some interested variable within the whole network, by only exchanging information with local neighbors. This interested variable can be a pose or attitude in coordination of robots [1, or a position in rendezvous [2, 3], or a velocity in flocking [4], or an oscillating frequency in synchronization [5], or an estimate of some parameter in sensor fusion [6]. Typically, for first-order systems the distributed consensus protocol is designed by a weighted averaging scheme as follows:

$$
x_{i}(k+1)=\sum_{j \in \mathcal{N}_{i}(k) \cup\{i\}} w_{i j}(k) x_{j}(k)
$$

for discrete-time systems, or

$$
\dot{x}_{i}=\sum_{j \in \mathcal{N}_{i}(t)} w_{i j}(t)\left(x_{j}(t)-x_{i}(t)\right)
$$

for continuous-time systems, where $\mathcal{N}_{i}$ denoted the neighbor set of agent $i$. The distributed consensus then can be achieved if the communication network satisfies some connectivity conditions. Based 
on this idea, a variety of control protocols have also been devised for MASs with different types of dynamics.

However, to implement the consensus protocol for practical MASs, we are still faced with a variety of challenges, especially different kinds of constraints in practice. Generally speaking, these constraints can be categorized as system-specific and task-specific constraints. The first type of constraints is inherited from the physical limits of practical systems: in the network level it can be the time delay and packet drops in communication, as well as the limited data rate in bandwidthlimited channel; it also can be the kinematic constraints and actuator limits of the individual system. The second type of constraints comes from the specific task requirement, e.g. consensus under set constraint which requires the final consensus value to fall within some given constraint set, or optimal consensus which requires the final consensus value to minimize some performance index or aggregate cost. Correspondingly, in this thesis we shall study two special cases of distributed consensus under constraints: quantized consensus and optimal consensus.

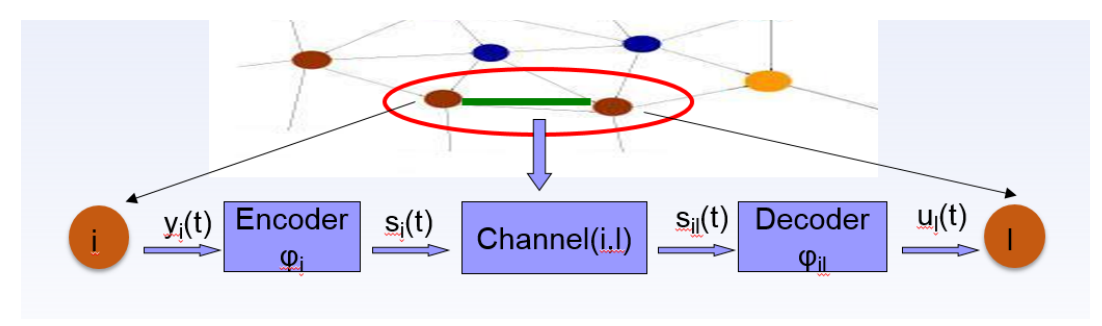

Figure 1.1: Workflow in quantized consensus.

As is well known, any communication channel has limited capacity and only finite bits of errorfree information can be transmitted per unit time. Therefore, any analog signal has to go through an encoding-decoding scheme for data quantization with a finite-bit quantizer, and the consensus protocol is designed based on the quantized data. Typically, the sender firstly encodes its state into symbolic data and sends the data over the communication channel, and then the receiver decodes the information to obtain an estimate of the sender's state with some error, which will be used in the control input (see Fig. 1.1 for an illustration). Furthermore, it is of theoretical and practical interest to obtain a sufficient data rate for quantized consensus, as well as the deciding factor of the sufficient data rate. It is known that for MASs with general linear system dynamics, the quantized consensus can be obtained with finite-bit information exchange 7 ; more specifically in the cases of first and second order integrators, the respective required data rate for consensus can be as low as 1 and 2 bits 8.9 . However, it is still an open problem about how many bits of data exchange suffice to achieve the quantized consensus, especially for general high-order dynamics with unmeasurable state; besides, it is not clear what determines the sufficient data rate. The first part of this thesis will try to provide an initial answer to this problem: to be detailed, we investigate the sufficient data rate of quantized consensus for two special kinds of high-order critical systems, respectively as $n$-th order 
integrators $(n \geq 3)$ and $2 m$-th order oscillators $(m \geq 1)$.

For the optimal consensus of MASs, it is usual to assign each agent with an individual cost and requires the whole system to achieve the consensus at the minimum of the aggregate cost. This problem is closely related with the consensus-based distributed optimization problem, whose algorithm is implemented in discrete-time iteration and it does not need to consider the dynamics of each agent. Various algorithms have been proposed for the distributed optimization problem, and it is intriguing and promising to implement these algorithms on practical physical systems such as robots and unmanned vehicles in an effort to optimize some performance index of the collective task. However, to this aim we need to accordingly adapt the corresponding distributed optimization algorithm to account for the physical limits of practical systems, as well as their dynamics. The second part of this thesis attempts to make an initial exploration in this direction. To be specific, we study the optimal consensus problem under set constraint for agents with single integrator dynamics respectively in continuous-time and discrete-time cases, and the optimal consensus problem of Euler-Lagrangian systems under kinematic constraints.

\subsection{Major Contributions and Outline}

In the subsequent two chapters we shall briefly review the relevant literature and preliminaries. The main body of the thesis is divided into two parts and spans from Chapter 4 to Chapter 8 , and we conclude the whole thesis and present some potential future work in Chapter 9. As follows are summarized the main content and contribution of Chapters 4 to 8.

- Part I Quantized consensus under data rate constraint

- Chapter 4 This chapter studies the quantized consensus problem of $n$-th order integrator MASs with only the first state variable being measurable. Motivated by the observer encoder/decoder design for the double integrator case studied in [9], we construct a similar encoding-decoding scheme to obtain a time-delayed estimate of the unmeasurable state variables, which is to be used in the consensus protocol. By employing perturbation techniques, we are able to not only design the control gains in an explicit form, but also conclude that it just needs $n$-bit information exchange to achieve the quantized consensus at an exponential convergence rate for MASs with $n$-th order integrator dynamics, if the communication network is fixed and connected. Our result not only generalizes the existing results for single and double integrator MASs, but also obtains the minimum data rate of quantized consensus for integrator systems among existing works. Moreover, the use of perturbation analysis is completely different from existing works on quantized consensus, in terms of 
both consensus analysis and data rate analysis. We further extend the result to the case when the system matrix of each agent only admits unit eigenvalues with single input, by applying the similar encoder/decoder and control protocol to a corresponding Luenberger observer. The final consensus value and an application to formation control problem are also discussed.

- Chapter 5 This chapter studies the quantized consensus problem of MASs with $2 m$-th order oscillator dynamics, which is another kind of critical high-order dynamics like the integrator. Each agent bears a system matrix which is a $2 m$-th order real Jordan block admitting $m$ identical pairs of conjugate poles on the unit circle; besides, it has a single input, and only the first state variable can be measured. In the absence of integrator structure, we first propose a novel encoding-decoding scheme to estimate the unmeasurable state variables based on the constructability of each individual system, and then design a consensus protocol based on the encoder/decoder outputs. The case of harmonic oscillators $(m=1)$ is first investigated under a directed communication topology which contains a spanning tree, while the general case of $m \geq 2$ is considered for a connected and undirected network. With the aid of perturbation analysis and combinatorics techniques, we not only achieve the quantized consensus at an exponential convergence rate, but also find an exact expression of sufficient data rate which is an integer between $m$ and $2 m$ depending on the frequency of the oscillator.

- Part II Optimal consensus under set constraint or kinematic constraints

- Chapter 6 This chapter studies the optimal consensus problem under a common set constraint for continuous-time single integrator MASs. To achieve the consensus at the optimum within the constraint set, three terms are included in the control protocol: local averaging, local projection vector, and local subgradient descent with a diminishing gain $\alpha(t)$. After modeling the whole system as a differential inclusion problem, we employ a strict non-smooth analysis to show the final convergence. Specifically, under a balanced communication network with uniformly joint spanning trees and bounded time-varying edge weights, we show that the constrained optimal consensus can be achieved when $\alpha(t)$ is non-integrable, which relaxes the corresponding condition in the distributed optimization literature. We further provide a convergence rate analysis for different types of $\alpha(t)$ when the aggregate cost is strongly convex.

- Chapter 7 This chapter studies the optimal consensus problem under a common set constraint for discrete-time single integrator MASs. As with the continuous-time case studied in Chapter 6, we design the control protocol based on local averaging, local projection vector, and local subgradient descent with a diminishing stepsize. Under similar conditions 
as in the continuous-time case, we show the optimal consensus within the constraint set, and provide a corresponding convergence rate analysis. Moreover, when the communication network is fixed, we further discuss the necessary conditions to ensure the optimal consensus without communication delays, as well as the convergence result in the presence of fixed communication delays.

- Chapter 8 This chapter studies the optimal consensus problem of MASs with heterogeneous EL dynamics under kinematic constraints. Specifically, the agents are required to reach the optimum position under bounded velocity and control inputs. Assuming an exact knowledge of nonlinearities in the EL equation and a priori estimate of the optimum, we first explore the case of double-integrator MASs by employing a quadratic Lyapunov function, and then extend the result to the case of EL dynamics by inverse dynamics control. Under a fixed and connected network, we show that the control gains can be properly selected to achieve an exponentially fast convergence while satisfying the bounded kinematic constraints. 



\section{Chapter 2}

\section{Literature Review}

Aiming to reach an agreement of certain quantities, the multi-agent consensus problem has a long history in the field of distributed computing [10]. The recent decade has witnessed a surge of research interest in the distributed multi-agent consensus within the control community. Actually, consensus poses as a basic problem in the cooperative control of MASs, and a distributed protocol is much more preferable than a centralized one due to its scalability with respect to the network size and robustness to the node/link failure, among other advantages. A variety of engineering applications has been found by employing the distributed multi-agent consensus protocol, e.g. coordination 1], flocking [4], formation control [11], rendezvous [12], distributed output regulation [13, distributed sensor fusion 14 and distributed optimization [15].

Basically, the design of a distributed consensus protocol depends on two key factors: the dynamics of individual agents and the consensus objective. Based on an averaging principle observed by Vesek [16], early works focused on achieving a weighted average of initial states for single integrators 1, 17, 19]. Later on, more complex dynamics was taken into account. For linear systems, second order dynamics cases were respectively considered as double integrators 20 23], or harmonic oscillators [24 26, and high-order integrator cases were also studied [27 29]. MASs with more general linear system dynamics were examined in $[7,30,31$. The distributed consensus with nonlinear dynamics was also explored, typically for nonlinear oscillators 32], nonholonomic mobile robots [33], and EL systems [34. Note that in the above works, the final consensus value is usually dependent on the specific dynamics as well as the initial state of each agent. Some other works are concerned about achieving consensus on general functions [35], or to track the average of reference inputs 36]. Moreover, in the case of distributed optimization or optimal consensus, the final consensus value is required to minimize an aggregate cost as the sum of individual costs [15. For a more comprehensive review on distributed multi-agent consensus, see survey papers 37, 39] and books [40,41. 
However, to implement the consensus protocol for practical MASs, various types of constraints have to be taken into account. Generally speaking, these constraints can be categorized into two groups: system-specific constraints and task-specific constraints. The first type of constraints is inherited from the physical limits of practical systems, e.g. communication delay [42, limited data rate in digital/analog channels [43, and kinematic constraints due to the actuator saturation [44. The second type of constraints comes from the task requirement, especially about the final consensus value which needs to fall within some particular constraint set or to minimize some performance index. In the interest of this thesis, we review recent relevant results on quantized consensus and optimal consensus in the following two sections.

\subsection{Quantized Consensus}

The recent research on quantized consensus can be categorized into two groups, depending on whether the state of each agent is modeled as integer-valued (quantized-valued) or real-valued. For agents with limited physical storage, their states are usually modeled as integer-valued, and the consensus can usually be achieved in finite time with an error of at most one quantization level from the initial average by using uniform quantizers. A large body of works are dedicated to applying gossip algorithms 43], i.e. selecting a single pair of neighboring agents to exchange information at each time slot. In an undirected and connected network, Kashyap et al. 45 designed a class of update rules to preserve the initial state sum by compensation and reduce the state difference of two activating agents, and showed that the average consensus (AC) can be achieved within one quantization level almost surely in finite time. This result was amended and extended in 46 to achieve the $\mathrm{AC}$ with exact agreement in a strongly connected network, by including surplus variables to help preserve the initial state sum and refining the update rule in the gossip algorithm. The convergence time was further analyzed in [47] for the special case when the graph is complete and each edge is activated with an equal probability. Note that the update rules in the above papers are designed to reduce the state difference of two selected agents and not given in an explicit form. With a specific update rule, 48 discussed the convergence time by employing the random walk theory, and obtained an upper bound of $O\left(N^{5}\right)$ in terms of the network size $N$. With the same update rule as in [45], [49] obtained a convergence time estimate of $O\left(N^{3} \log N\right)$ under the assumption that the pair of updating nodes is selected by a Poisson process. 50 further derived a more explicit expression of the upper bound as $O(N M D \log N)$, where $M$ and $D$ are respectively the number of edges and the diameter of the network, with the application of a conservative mixing strategy.

Most works on quantized consensus focus on real-valued agents, where the consensus is achieved asymptotically or with steady-state error. Besides uniform quantizers, logarithmic and probabilistic quantizers are also used for quantization. The convergence analysis is carried out in a probabilistic 
or deterministic fashion, depending on the problem setup. By employing probabilistic quantizers which yields a uniformly distributed zero-mean quantization error, 51 achieved the consensus almost surely at one of the quantization values, with an expectation equal to the initial average. Similar quantizers are also used in 52 to achieve the probabilistic $\mathrm{AC}$ in networks with random link failures. As in the case of integer-valued agents, gossip algorithms are also applied to study the AC problem. Inspired by the work in [45], 53] proposed a specific compensating rule with a mixing parameter $p=1 / 2$ respecitvely for uniform and probabilistic quantizers, and showed that the $\mathrm{AC}$ can be achieved within one quantization level, respectively in the sense of almost sure convergence or asymptotic mean square error. These results have been generalized in [54 and [55]. Specifically, for uniform quantizers, 54 extended the almost sure convergence to general quantizers with $p \in(0,1 / 2]$ and discussed the convergence rate; for probabilistic quantizers, 55] extended the corresponding result to almost sure convergence with $p \in(0,1)$, and pointed out that the minimum asymptotic mean square error is minimized at $p=1 / 2$. By modifying the basic weighted average scheme of AC with a compensation of quantized information, [56] used probabilistic quantizers to achieve the $\mathrm{AC}$ in the mean square sense via sequence averaging, and 57] used logarithmic quantizers to achieve the AC with steady-state error which decreases with an increased quantization accuracy.

The above results only focus on achieving the $\mathrm{AC}$ with limited data rate, and they are not concerned about reducing the data rate in transmission. Many works have been dedicated in this direction by using a dynamic encoding/decoding scheme with uniform quantizers. To be detailed, the dynamic scheme is able to achieve a higher quantization accuracy with the same bits of data, by relating the current state with previous quantization outputs. Usually, the information to be quantized is a scaled innovation of the current state from the last quantization output, and the problem amounts to the choice of the quantization scale. A deterministic zoom-in zoom-out quantizer was proposed in [58 with an adaptively tuned scale, and the convergence to the initial average was proven by applying the small gain theorem. A similar idea was developed in 59 from a probabilistic perspective, where the quantization range is chosen to be exponentially decaying by considering the mean square innovation, assuming the quantization error to be white noise. With a given bound of initial states, 8 employed a zoom-in quantizer with an exponentially decaying scale function, and it was shown that one bit of data exchange suffices for the exponentially fast convergence in a fixed and connected network by properly selecting control parameters. This result was later extended to the case of a fixed and balanced network which containes a spanning tree 60. In a connected network with time-varying link failures, the $\mathrm{AC}$ can be achieved by 5 quantization levels if the interval of any link failure is bounded. 61 obtained a similar result for a directed network with a spanning tree, without specifying the consensus value. Olshevsky 62$]$ considered using different hyperharmonic series $\left(1 / t^{p}, 0<p<1\right)$ respectively as the quantization scales and update stepsizes. By carefully screening the information received from neighbors and properly selecting parameters, it was shown 
that one bit of data is enough to guarantee the $\mathrm{AC}$ in polynomial time without knowing the bound of the initial states.

Besides the works on the AC for first order systems, the quantized consensus with high-order dynamics is also considered. Under consensusability conditions, it has been shown in 7 that quantized consensus can be achieved with a limited data rate for general linear system dynamics with a fully measurable state. In the case of a partially measurable state, the encoder/decoder should also serve as an observer to estimate the unmeasurable state. This method was first developed in 9 to study the quantized consensus of double-integrator MASs, where only the first state variable can be measured. Compared with the first order case, it needs an addditional bit of data to cover the quantization range of the scaled innovation. By employing perturbation techniques, similar results can be obtained for some special high-order critical systems, such as integrator and oscillator systems respectively studied in 63 and 64 (see subsequent Chapters 4 and 55. However, for MAS with general high-order dynamics, it is still an open problem about how many bits of information exchange suffice for the quantized consensus.

\subsection{Optimal Consensus}

As the name suggests, optimal consensus means to reach an agreement while optimizing a particular performance index. It makes practical sense to consider the problem for MASs in order to improve the performance of collective tasks. Moreover, it also provides another insight into the original consensus problem: for instance, the $\mathrm{AC}$ problem is equivalent to finding a common estimate to minimize the mean square error in a sensor network. In the MAS context, the performance index is usually given as the sum of individual costs, and it is the convex cost that has attracted the most attention. Below we first review some recent results from the consensus-based distributed optimization problem, which is closely related with the optimal consensus problem.

The research on the distributed optimization can be dated back to 1980s [65], which has found widespread applications in big data analysis [66, distributed learning 67], and source localization [68. But it was only until recently that researchers became interested in applying the consensus mechanism to solve distributed optimization problems which assume a separable cost. In this case, different agents have their own private cost and decision variable, and they need to agree upon the decision variable and minimize the aggregate cost simultaneously. In general, two consensus-based approaches are proposed to solve the problem. The first approach actively attaches the weighted averaging scheme of decision variables to a centralized optimization algorithm. In this light, consensus is prioritized so that the convergence of the optimization algorithm can be analyzed in a nearly centralized fashion. For agents with set constraints, Nedic et al. 69] proposed a distributed projected subgradient algorithm and 
showed the convergence when the constraint set is identical and the balanced network is periodically strongly connected. The result was later extended in 70 to the case of nonidentical constraints. By employing push-sum techniques, the case of unbalanced works was studied in 71 . The distributed dual averaging method was studied in 72 and 73 respectively for balanced and unbalanced networks. By embedding an inner loop of averaging steps, $[74$ and $[75]$ respectively proposed the distributed version of fast gradient methods and fast proximal-gradients with the use of Nesterov-type acceleration. By applying saddle point methods, 76 studied the case with inequality constraints, and 77] studied the case with both equality and inequality constraints. [78] adopted slow and fast time scales respectively for the update of states and Newton directions. In the above works, an exact convergence to the optimum requires either diminishing stepsizes of optimization force as in the gradient-based algorithms 69 71, 77, or a drastically increasing communication burden for averaging steps with each iteration 74, 75.78. Both cases lead to a compromised convergence when compared with the corresponding centralized algorithms.

On the other hand, the consensus requirement can be also viewed as an equality constraint and naturally gives rise to the dual decomposition. A popular approach is the alternating method of multipliers (ADMM) 79 which is essentially an augmented Lagrangian method. When formulated properly, ADMM can be implemented in a distributed form and achieves linear convergence rate for strongly convex costs whose gradients are Lipschitz continuous 80, 81. To mitigate the issue of successive minimization of primal update in the original ADMM, inexact methods were also developed recently to approximate the primal update, e.g. a linear approximation 82 or a quadratic approximation 83. A class of augmented Lagrangian methods was considered in 84 with four different kinds of inexact algorithms. Note that the above methods are only applicable to a fixed network based on primal-dual techniques. It should also be mentioned that the recent trend is to use constant stepsizes in gradient-based algorithms, by replacing the local gradient with an approximate average gradient of the aggregate cost 85,86 . As pointed out in [87, the consensus on the average gradient may be regarded as a dual update; however, the whole analysis can be conducted in primal perspective and applied to a time-varying network, which is different from the dual decomposition approach. See 87,88 for more details.

To achieve the optimal consensus of practical MASs, it is necessary to consider the corresponding continuous-time dynamics. Moreover, the application of nonlinear techniques in the continuous-time formulation also provides a complementary viewpoint to the distributed optimization problem. Based on dual decomposition, Wang and Elia proposed a saddle-point algorithm to solve an unconstrained optimization problem in 89], whose formal convergence analysis was provided in [90. This algorithm was modified in 91 by treating the dual variable as an integral feedback to correct the consensus error. The case with a common set constraint was considered in 92 as an extension of the projected subgradient algorithm (also see Chapter 6 later). The same problem was studied in 93] for second- 
order dynamics, and the case of different set constraints was investigated in 94]. When there exist additional local inequality and equality constraints, 95 proposed a proportional-integral protocol to solve the problem. 96] dealt with coupling inequality constraints by using saddle-point dynamics with averaging of primal and dual variables. 97 investigated the case with different set constraints and coupling equality constraints for resource allocation problems. Some works took into account more complex dynamics, such as the high-order integrators 98 and the EL systems 99 . Still, there remains much to be explored about the combination of system dynamics and optimization in MASs. 


\section{Chapter 3}

\section{Preliminaries}

\subsection{Graph Theory}

The communication among different agents (nodes) of an MAS can be modeled as a directed graph $\mathcal{G}$, which consists of a node set $\mathcal{N}=\{1, \ldots, N\}$ and an edge set $\mathcal{E}=\{(i, j): i, j \in \mathcal{N}\}$, where $(i, j) \in \mathcal{E}$ iff node $j$ can receive information from node $i$. For node $i, \mathcal{N}_{i}^{+}=\{j:(j, i) \in \mathcal{E}\}$ and $\mathcal{N}_{i}^{-}=\{j:(i, j) \in \mathcal{E}\}$ respectively denote its in-neighbors and out-neighbors, which will be denoted as $\mathcal{N}_{i}$ when they coincide.

Moreover, a non-negative weight matrix $W=\left[w_{i j}\right] \in \mathbb{R}_{\geq 0}^{N \times N}$ can be assigned to a graph $\mathcal{G}$, and $w_{i j}>0$ iff $(j, i) \in \mathcal{E}$. If $W$ is symmetric, then $\mathcal{G}$ is said to be undirected. If $W \mathbf{1}_{N}=W^{\prime} \mathbf{1}_{N}$, then $\mathcal{G}$ is said to be balanced. Besides, given a non-negative matrix $P \in \mathbb{R}_{\geq 0}^{N \times N}$, a graph $\mathcal{G}(P)=\{\mathcal{N}, \mathcal{E}(P)\}$ can be associated by letting $\mathcal{E}=\{(i, j): P(i, j)>0\}$.

A path from node $n_{0}$ to node $n_{d}$ is defined by a sequence $n_{1}, \ldots, n_{d} \in \mathcal{G}$ satisfying $\left(n_{0}, n_{1}\right),\left(n_{1}, n_{2}\right), \ldots,\left(n_{d-1}, n_{d}\right) \in \mathcal{E} . \mathcal{G}$ is said to contain a spanning tree if there exists a node $i$ connected to any other node $j$ by a path. In this case, the node $i$ is also called a root of $\mathcal{G}$. $\mathcal{G}$ is said to be strongly connected if there always exists a path between any two different nodes. A balanced graph with a spanning tree is strongly connected. An undirected graph $\mathcal{G}$ is always balanced, and it is said to be connected if it contains a spanning tree.

When the communication topology is time-varying, $\mathcal{G}$ is also used to denote the map $\mathcal{G}:\left[t_{0}, \infty\right) \rightarrow$ $\mathcal{Q}$ where the set $\mathcal{Q}$ comprises all the possible weighted graphs with an identical node set $\mathcal{N}$, and $\mathcal{G}(t)=\{\mathcal{N}, \mathcal{E}(t)\}$ is the graph at time $t$. The joint graph over time span $\left[t_{1}, t_{2}\right]$ is given by $\mathcal{G}\left[t_{1}, t_{2}\right]=$ $\left\{\mathcal{N}, \cup_{t=t_{1}}^{t=t_{2}} \mathcal{E}(t)\right\}$. Similar notations apply to $\mathcal{G}\left[t_{1}, t_{2}\right)$ and $\mathcal{G}\left(t_{1}, t_{2}\right]$. If there exists a constant $T>0$ such that $\mathcal{G}[t, t+T]$ has a spanning tree for any $t$, then $\mathcal{G}$ is said to uniformly contain joint spanning trees. Similar definitions apply to uniformly jointly (strongly) connected graphs. 
For a weighted graph $\mathcal{G}=\{\mathcal{N}, \mathcal{E}, W\}$, the Laplacian matrix $L=\left[l_{i j}\right]$ is defined by $l_{i i}=\sum_{j \in \mathcal{N}_{i}} w_{i j}$ and $l_{i j}=-w_{i j}$ for $i \neq j$. The degree matrix of $\mathcal{G}$ is given by $D=\operatorname{diag}\left\{D_{1}, \ldots, D_{N}\right\}$ with $D_{i}=$ $\sum_{j \in \mathcal{N}_{i}^{+}} w_{i j}, i=1, \ldots, N$. $L$ can be used to examine the connectivity from an algebraic viewpoint. Noticing that $L \mathbf{1}_{N}=0, L$ has at least one zero eigenvalue; moreover, all non-zero eigenvalues of $L$ have positive real parts as a result of Gershgorins disc theorem [100]. The zero eigenvalue has an algebraic multiplicity of one iff $\mathcal{G}$ has a spanning tree [101]. If $\mathcal{G}$ is undirected, then all the eigenvalues of $L$ are non-negative and can be further arranged as $0=\lambda_{1}<\lambda_{2} \leq \cdots \leq \lambda_{N}$ if $\mathcal{G}$ is connected.

\subsection{Stochastic Matrix Theory}

The theory of stochastic matrices is a basic tool in solving consensus problems of discrete-time multiagent systems. A stochastic matrix $P \in \mathbb{R}_{\geq 0}^{N \times N}$ is a non-negative matrix with row sums all equal to 1 , i.e. $P \mathbf{1}_{N}=\mathbf{1}_{N}$. It is said to be indecomposable and aperiodic (SIA) if there exists $\nu \in \mathbb{R}^{N}$ such that $\lim _{k \rightarrow \infty} P^{k}=\mathbf{1} \nu^{\prime}$. Noticing that $P$ always has a unit eigenvalue and the magnitude of any eigenvalue is not greater than $1, P$ is SIA iff the unit eigenvalue is the only eigenvalue of maximum modulus. The following lemma provides a sufficient condition to determine whether $P$ is SIA.

Lemma 3.1. [102] Let $P$ be a stochastic matrix. If $\mathcal{G}(P)$ contains a spanning tree rooted at $r$ and $P(r, r)>0$, then $P$ is $S I A$.

The next lemma is useful in analyzing the consensus convergence in the presence of communication delays.

Lemma 3.2. [102] Denote $P=P_{0}+P_{1}+\cdots+P_{d}$ with each $P_{i} \in \mathbb{R}_{\geq 0}^{N \times N}$. Let

$$
M=\left[\begin{array}{ccccc}
P_{0} & P_{1} & \cdots & P_{d-1} & P_{d} \\
I & & & & \\
& I & & & \\
& & \ddots & & \\
& & & I &
\end{array}\right] .
$$

If $\mathcal{G}(P)$ has a spanning tree, then $\mathcal{G}(M)$ has a spanning tree. Furthermore, if $P$ is stochastic and $P_{0} \geq \mu I$ holds entry-wise for some $\mu>0$, then $M$ is $S I A$.

Another lemma is introduced before closing this section.

Lemma 3.3. Let $P$ be a stochastic matrix and $\nu$ be a left eigenvector with respect to the unit eigenvalue. Then it holds that $P^{\prime} \Lambda_{\nu} P \leq \Lambda_{\nu}$, where $\Lambda_{\nu}=\operatorname{diag}\{\nu\}$. 
Proof. We just need to prove that $\Lambda_{\nu}-P^{\prime} \Lambda_{\nu} P$ is a diagonally dominant matrix. Actually $\nu$ is a non-negative vector of $P$, and $P^{\prime} \Lambda_{\nu} P$ is a non-negative matrix. Moreover, the row sum vector of $P^{\prime} \Lambda_{\nu} P$ is found as $P^{\prime} \Lambda_{\nu} P \mathbf{1}=P^{\prime} \nu=\nu$, which establishes the result.

\subsection{Convex Analysis}

\subsubsection{Convex Sets}

A set $\mathcal{C} \subset \mathbb{R}^{m}$ is convex if $\lambda x+(1-\lambda) y \in \mathcal{C}$ for any $x, y \in \mathcal{C}$ and $0 \leq \lambda \leq 1$. For a closed convex set $\mathcal{C} \in \mathbb{R}^{m},|x|_{C} \triangleq \inf _{c \in C}\|x-c\|$ is the distance between $x$ and $C$, and $P_{\mathcal{C}}(x) \in \mathcal{C}$ is the projection of $x$ onto $\mathcal{C}$, uniquely satisfying $\left\|x-P_{\mathcal{C}}(x)\right\|=|x|_{\mathcal{C}}$. The projection operator has the following properties 103 .

Lemma 3.4. Given a closed convex set $\mathcal{C} \subset \mathbb{R}^{m}$ and $x \in \mathbb{R}^{m}$, it holds that

$$
\left\langle P_{\mathcal{C}}(x)-x, P_{\mathcal{C}}(x)-y\right\rangle \leq 0, \forall y \in \mathcal{C} .
$$

Lemma 3.5. Given a closed convex set $\mathcal{C} \subset \mathbb{R}^{m}$ and $x, y \in \mathbb{R}^{m}$, it holds that

$$
\left\|P_{\mathcal{C}}(x)-P_{\mathcal{C}}(y)\right\| \leq\|x-y\| .
$$

The next lemma estimates the inner product involving the projection vector, which is reminiscent of Cauchy-Schwarz inequality in a convex context. It can also be found in 104, Lemma 13], and here we provide a more concise and intuitive proof.

Lemma 3.6. Given a closed convex set $\mathcal{C} \subset \mathbb{R}^{m}$ and $x, y \in \mathbb{R}^{m}$, we have

$$
\left\langle x-P_{\mathcal{C}}(x), y-x\right\rangle \leq|x|_{\mathcal{C}}\left(|y|_{\mathcal{C}}-|x|_{\mathcal{C}}\right) .
$$

Proof. If $|x|_{\mathcal{C}}=0$, or equally $x=P_{\mathcal{C}}(x) \in \mathcal{C}$, the result is obvious. Hence we only need to focus on the case of $|x|_{\mathcal{C}} \neq 0$.

Define $r=\frac{x-P_{\mathcal{C}}(x)}{|x|_{\mathcal{C}}}$ and $H=\left\{z:\left\langle r, z-P_{\mathcal{C}}(x)\right\rangle=0\right\}$. By the separation theorem of convex sets, $H$ is a hyperplane supporting $\mathcal{C}$ at $P_{\mathcal{C}}(x)$ and $\mathcal{C} \subset H^{-}$, where $H^{-}=\left\{z:\left\langle r, z-P_{\mathcal{C}}(x)\right\rangle \leq 0\right\}$.

Moreover, denote $y_{H}=\left\langle r, y-P_{\mathcal{C}}(x)\right\rangle$. Noticing that $\langle r, y-x\rangle=\left\langle r, y-P_{\mathcal{C}}(x)+P_{\mathcal{C}}(x)-x\right\rangle=$ $y_{H}-x_{H}$ and $x_{H}=|x|_{\mathcal{C}}$, the proof is completed by observing that $y_{H}=d(y, H) \leq|y|_{\mathcal{C}}$ when $y_{H} \geq 0$. 


\subsubsection{Convex Functions}

A function $f: \mathbb{R}^{m} \rightarrow \mathbb{R} \cup\{+\infty\}$ is convex if for any $x, y \in \mathbb{R}^{m}$ and $0<\lambda<1$, it holds that

$$
f(\lambda x+(1-\lambda) y) \leq \lambda f(x)+(1-\lambda) f(y)
$$

$f$ is strictly convex when 3.4 holds as a strict inequality for $x \neq y$. The domain of $f$ is defined by the nonempty convex set $\operatorname{dom} f=\left\{x \in \mathbb{R}^{m}: f(x)<+\infty\right\}$. If $\operatorname{dom} f=\mathbb{R}^{m}, f$ is said to be finite. If $\lim _{\|x\| \rightarrow \infty}|f(x)|=\infty$, then $f$ is said to be coercive. For any convex set $\mathcal{C}$, set distance function $|\cdot|_{\mathcal{C}}$ is convex and finite.

The subdifferential of a convex function $f$ at $x$ is defined as the set

$$
\partial f(x)=\{g: f(y) \geq f(x)+\langle g, y-x\rangle, \forall y\}
$$

with the element $g \in \partial f(x)$ called a subgradient of $f$ at $x$. If $f$ is finite, then $\partial f(x)$ is a nonempty and compact convex set everywhere. Moreover, the set-valued map $\partial f$ is upper semi-continuous:

Lemma 3.7. [103] For any $\varepsilon>0$, there exists $\delta>0$ such that $\partial f(y) \subset \partial f(x)+B(0, \varepsilon)$ when $y \in B(x, \delta)$.

The subdifferential of $\varphi(t)=f(x+t(y-x))$ can be found in the lemma below, reminiscent of the directional derivative for a differentiable $f$ :

Lemma 3.8. 103] $\partial \varphi(t)=\left\{\langle g, y-x\rangle: g \in \partial f\left(x_{t}\right)\right\}$; or symbolically put as $\partial \varphi(t)=\left\langle\partial f\left(x_{t}\right), y-x\right\rangle$.

The convex function $f$ is said to be $\beta$-strongly convex over some convex set $\mathcal{C}$, if there exists $\beta>0$ such that for $\forall \lambda \in(0,1)$

$$
f(\lambda x+(1-\lambda) y) \leq \lambda f(x)+(1-\lambda) f(y)-\frac{1}{2} \beta \lambda(1-\lambda)\|x-y\|^{2}, \forall x, y \in \mathcal{C}
$$

The following lemma provides a quadratic lower bound of $f$ :

Lemma 3.9. Let $\mathcal{C}$ be a convex and closed set. If $f$ is $\beta$-strongly convex over $\mathcal{C}$, then there exists $x^{*} \in \mathcal{C}$ such that

$$
f(x)-f\left(x^{*}\right) \geq \frac{\beta}{2}\left\|x-x^{*}\right\|^{2}, \forall x \in \mathcal{X} .
$$

Proof. By Theorem 6.1.2 of 103, the strong convexity 3.5 is equivalent to

$$
f(x) \geq f(y)+\langle g(y), x-y\rangle+\frac{\beta}{2}\|x-y\|^{2}, \forall g(y) \in \partial f(y), \forall x, y \in \mathcal{C} .
$$

Furthermore, we know that $f$ has a unique minimum $x^{*} \in \mathcal{C}$, which dictates the existence of $g\left(x^{*}\right) \in$ $\partial f\left(x^{*}\right)$ such that $\left\langle g\left(x^{*}\right), x-x^{*}\right\rangle \geq 0$. Now we obtain (3.6) by letting $y=x^{*}$ in 3.7 . 
Part I

\section{Quantized Consensus}

\section{under Data Rate Constraint}




\section{Chapter 4}

\section{Quantized Consensus of $n$-th Order Integrator Systems under Data Rate Constraint}

Just as the minimum data rate problem in networked control [105], it is of paramount theoretical importance to obtain a sufficient data rate in quantized consensus, and understand the corresponding deciding factor. For the purpose of communication saving, it is also of pratical interest to achieve the quantized consensus with a low data rate. It has been respectively shown in 8 and 9 that for first and second order integrators, one and two bits of information exchange suffice to achieve the quantized consensus at an exponential convergence rate. In this light, it is natural to ask whether the above results can be extended to general $n$-th order integrators. Moreover, high-order integrators are also very important both in practice and theory. Firstly, higher-order dynamics exist in many mechanical systems, such as the inclusion of acceleration and jerk for generating a smooth trajectory of UAVs 106. Secondly, integrators are the simplest critical systems, for which the study of quantized consensus can pave the way to more general linear dynamics. Actually, as can be seen later in Section 4.3. the result obtained for $n$-th order integrator systems can be applied to a more general case, when each agent is controllable and observable with single input and single output, and its system matrix only admits unit eigenvalues.

It should be pointed out that it is not a straightforward extension from double integrators to general $n$-th order integrators. As has been shown in the double integrator case 9, to obtain the result of using 2-bit information exchange for quantized consensus, it needs an explicit characterization of the influence of control input on the convergence of each state component. Therefore, it requires not 
only an explicit design of control input, but also an accurate estimate of the convergence rate, which is extremely difficult for high-order systems. Fortunately, with the use of perturbation techniques we can attain both goals and successfully generalize previous results: for $n$-th order integrator systems with only the first state variable measurable, it suffices to use $n$ bits of information exchange to achieve the quantized consensus at an exponential rate.

The rest of the chapter is organized as follows. The problem is first formulated in Section 4.1. Then the analysis of leaderless and leader-following quantized consensus of $n$-th order integrator systems is given in Section 4.2. Later in Section 4.3, similar results are obtained for a more general case when the system only admits unity eigenvalues with single input. The consensus value and application to formation control are discussed in Section 4.4 with a numerical example provided in Section 4.5. We conclude the chapter in Section 4.6. with the proofs of the main lemmas presented in Section 4.7

\subsection{Problem Formulation}

Consider an MAS in the form of $n$-th order integrator dynamics with only the first state variable being measurable as below:

$$
\left\{\begin{aligned}
x^{i}(t+1) & =A^{*} x^{i}(t)+b^{*} u_{i}(t) \\
y_{i}(t) & =c^{*} x^{i}(t),
\end{aligned}\right.
$$

where $A^{*}=J_{1, n}, b^{*}=[0, \ldots, 0,1]^{\prime}$ and $c^{*}=[1,0, \ldots, 0]$. zHere $x^{i}(t)=\left[x_{i 1}(t), \ldots, x_{i n}(t)\right]^{\prime} \in \mathbb{R}^{n}$, $y_{i}(t), u_{i}(t) \in \mathbb{R}$, represent the state, output and input of agent $i$ for $i=0,1, \ldots, N$, respectively. In particular, for the leader-following consensus, the agent 0 is deemed as the only leader agent with no control input, that is, $u_{0}(t) \equiv 0$. For the leaderless case, the agent 0 is absent and $i=1, \ldots, N$.

In the sequel we will investigate the leaderless and leader-following consensus under a limited data rate. Due to the finite capacity in digital/analog channels, signals communicated between agents need to be encoded before transmission and then decoded at receivers. The finite-level uniform quantizer $q_{t}(\cdot)$ adopted in our encoding scheme takes the form of

$$
q_{t}(y)=\left\{\begin{array}{l}
0,-1 / 2<y<1 / 2 \\
k, \frac{2 k-1}{2} \leq y<\frac{2 k+1}{2}, k=1, \ldots, M(t)-1 \\
M(t), y \geq \frac{2 M(t)-1}{2} \\
-q_{t}(-y), y \leq-1 / 2
\end{array}\right.
$$

where $M(t) \in \mathbb{N}^{+}$. 
Remark 4.1. Clearly, the quantization levels of $q_{t}(\cdot)$ sum up to $2 M(t)+1$. Demanding that agent $i$ send out no signal when the output is zero, the required data rate to represent the output of the quantizer is given by $\left\lceil\log _{2}(2 M(t))\right\rceil$.

The problem of distributed leaderless quantized consensus is solved if we can design a distributed control protocol for each agent, based on the outputs of its encoder and decoders, such that the states of all the agents reach the following agreement asymptotically:

$$
\lim _{t \rightarrow \infty}\left(x^{i}(t)-x^{l}(t)\right)=0, \quad i, l=1, \ldots, N
$$

Similarly, the problem of distributed leader-following consensus is solved if we can find a distributed control protocol for each agent such that the states of the follower agents can track that of the leader agent asymptotically:

$$
\lim _{t \rightarrow \infty}\left(x^{i}(t)-x^{0}(t)\right)=0, \quad i=1, \ldots, N
$$

In the following two sections, we aim to solve the leaderless/leader-following consensus problem with quantized neighbor-based control, and investigate how many bits of information are needed to be exchanged for the consensus. We first solve the consensus problem for the special case of $n$-th order integrator systems with partially measurable state in Section 4.2, and then extend it to address the general case in Section 4.3 , with the help of a similarity transformation and state observers.

\subsection{Quantized Consensus and Data Rate for $n$-th Order Inte- grator Dynamics}

Basically, to solve the quantized consensus under a limited data rate for MASs with unmeasurable state variables, we are faced with the following three challenges: firstly, design an observer-based encodingdecoding scheme with a finite-bit quantizer; secondly, design a distributed control law based on the outputs of the encoding-decoding scheme; finally, analyze the sufficient data rate for the quantized consensus. In the following sections we will see how to address these three challenges, and we start with the observer-based encoder-decoder design in the next section.

\subsubsection{Observer-based Encoding-Decoding Scheme}

The foremost task of quantized consensus under a limited data rate is to design an encoding-decoding scheme with a finite-bit quantizer. Noticing that the system state may be unbounded for an integrator, we choose the quantization input as a scaled innovation of the output, which generalizes the 
encoding-decoding scheme of the double-integrator case studied in [9]. Different from a straightforward quantization of the possibly unbounded output which needs an unlimited data rate, the innovation of the output can be bounded with an accurate estimation, which gives rise to a finite-bit quantizer; on the other hand, with an exponentially decaying scaling function, an asymptotic estimation of the output (as well as the other unmeasurale state variables) can be achieved with decaying error, as long as the quantizer is unsaturated. Specifically, we design an observer-based encoding-decoding scheme as below.

Based on 4.1 we propose a finite memory encoder $\varphi_{i}$ for each agent (including the leader):

$$
\begin{aligned}
& t=1, \ldots, n-1:\left\{\begin{aligned}
\hat{x}_{i 1}(0) & =\hat{x}_{i 2}(0)=\hat{x}_{i 3}(1) \cdots=\hat{x}_{i n}(n-2)=0, \\
s_{i}(t) & =q_{t}\left(\frac{y_{i}(t)-\sum_{j=1}^{t} \hat{x}_{i j}(t-1)}{p(t-1)}\right), \\
\hat{x}_{i, t+1}(t) & =\hat{x}_{i, t+1}(t-1)+p(t-1) s_{i}(t), \\
\hat{x}_{i, t}(t) & =\hat{x}_{i, t}(t-1)+\hat{x}_{i, t+1}(t), \\
& \vdots \\
\hat{x}_{i 1}(t) & =\hat{x}_{i 1}(t-1)+\hat{x}_{i 2}(t)
\end{aligned}\right. \\
& t \geq n:\left\{\begin{aligned}
s_{i}(t) & =q_{t}\left(\frac{y_{i}(t)-\sum_{j=1}^{n} \hat{x}_{i j}(t-1)}{p(t-1)}\right) \\
\hat{x}_{i n}(t) & =\hat{x}_{i n}(t-1)+p(t-1) s_{i}(t) \\
\hat{x}_{i, n-1}(t) & =\hat{x}_{i, n-1}(t-1)+\hat{x}_{i n}(t) \\
& \vdots \\
\hat{x}_{i 1}(t) & =\hat{x}_{i 1}(t-1)+\hat{x}_{i 2}(t)
\end{aligned}\right.
\end{aligned}
$$

where $\hat{x}_{i j}(t)$ are the outputs of the encoder and

$$
p(t)=p_{0} \gamma^{t}, 0<\gamma<1
$$

is an exponentially decaying scaling function with two design parameters $p_{0}$ and $\gamma$.

After $s_{i}(t)$ is received by one of agent $i$ 's neighbors, say $l \in \mathcal{N}_{i}$, a decoder $\varphi_{i l}$ will be activated:

$$
t=1, \ldots, n-1:\left\{\begin{aligned}
\hat{x}_{i l 1}(0) & =\hat{x}_{i l 2}(0)=\hat{x}_{i l 3}(1) \cdots=\hat{x}_{i l n}(n-2)=0, \\
\hat{x}_{i l, t+1}(t) & =\hat{x}_{i l, t+1}(t-1)+p(t-1) s_{i}(t), \\
\hat{x}_{i l t}(t) & =\hat{x}_{i l, t}(t-1)+\hat{x}_{i l, t+1}(t), \\
& \vdots \\
\hat{x}_{i l 1}(t) & =\hat{x}_{i l 1}(t-1)+\hat{x}_{i l 2}(t) ;
\end{aligned}\right.
$$




$$
t \geq n:\left\{\begin{aligned}
\hat{x}_{i l n}(t) & =\hat{x}_{i l n}(t-1)+p(t-1) s_{i}(t), \\
\hat{x}_{i l, n-1}(t) & =\hat{x}_{i l, n-1}(t-1)+\hat{x}_{i l n}(t), \\
& \vdots \\
\hat{x}_{i l 1}(t) & =\hat{x}_{i l 1}(t-1)+\hat{x}_{i l 2}(t) .
\end{aligned}\right.
$$

Remark 4.2. From 4.5)-(4.9), we have

$$
\hat{x}_{i l 1}(t) \equiv \hat{x}_{i 1}(t), \ldots, \hat{x}_{i l n}(t) \equiv \hat{x}_{i n}(t),
$$

for $l \in \mathcal{N}_{i}, i=0,1, \ldots, N$. Namely, the encoder output of agent $i$ coincides with the decoder output of agent $i$ by agent $j$, demonstrating the synchronization between the sender and the receiver. If there is no quantization, or equally $q_{t}(\cdot)$ is an identity map, $\hat{x}_{i 1}(t)=x_{i 1}(t)$. Moreover, by (4.5) and (4.9) we have $\hat{x}_{i j}(t)=\hat{x}_{i, j-1}(t)-\hat{x}_{i, j-1}(t-1), j=2, \ldots, n$, indicating that $\hat{x}_{i j}(t)$ can be viewed as an estimate of $x_{i j}(t-j+1)$. Hence the input to the quantizer at time $t$ can be viewed as a scaled innovation.

Remark 4.3. Alternatively, we can take a look at the above encoder from another viewpoint. By ignoring the quantization effect, it is readily seen that

$$
y_{i}(t)-\sum_{j=1}^{n} \hat{x}_{i j}(t-1) \approx u_{i}(t-n), t \geq n .
$$

Accordingly, the neighbor agent $l$ can produce an estimate of the delayed control input $u_{i}(t-n)$ based on the received signal $s_{i}(t)$, and update the corresponding estimate of the state of agent $i$ with time delays. Intuitively speaking, if the control input is sufficiently small, the quantizer output can produce a good estimate of the state with fewer quantization levels. This motivates us to propose a distributed control protocol with low gains later. Also, it should be pointed out that such a design of encodingdecoding scheme has taken into consideration the special structure of the integrator dynamics and the fact that the first state variable is measurable, while for the general case in Section 4.3 we have to resort to a different kind of state observers.

Remark 4.4. The estimation error of $x_{i j}(t-j+1)$ is related to the scaling function and quantization error. Denote

$$
d_{i}(t)=\left\{\begin{array}{l}
\frac{y_{i}(t)-\sum_{j=1}^{t} \hat{x}_{i j}(t-1)}{p(t-1)}, t=1,2, \ldots, n-1 \\
\frac{y_{i}(t)-\sum_{j=1}^{n} \hat{x}_{i j}(t-1)}{p(t-1)}, t \geq n .
\end{array}\right.
$$

Then $\Delta_{i}(t)=s_{i}(t)-d_{i}(t)$ is the quantization error, and $e_{i j}(t-j+1)=\hat{x}_{i j}(t)-x_{i j}(t-j+1)$ is the 
estimation error for $x_{i j}(t-j+1)$. By (4.1), 4.5), and (4.9), we get

$$
\left\{\begin{aligned}
e_{i 1}(t) & =p(t-1) \Delta_{i}(t), t \geq 1 \\
e_{i 2}(t-1) & =e_{i 1}(t)-e_{i 1}(t-1) \\
& \vdots \\
e_{i n}(t-n+1) & =e_{i, n-1}(t-n+2)-e_{i, n-1}(t-n+1) .
\end{aligned}\right.
$$

By induction it follows that

$$
\sum_{j=1}^{\tau} e_{i j}(t-j+1)=\sum_{j=1}^{\tau} C(\tau, j)(-1)^{j-1} e_{i 1}(t-j+1), \tau=1,2, \ldots, n
$$

where $C(\tau, j)$ is the number of $j$-combinations from a given set of $\tau$ elements. (4.13) will be used frequently in convergence analysis of consensus.

\subsubsection{Leaderless Consensus}

In the leaderless consensus case, based on the outputs of the encoder and decoders, the distributed control law is given as $u(t)=\left[u_{1}(t), \ldots, u_{N}(t)\right]^{\prime}$ with

$$
u_{i}(t)=\left\{\begin{array}{l}
0, \quad t=0,1, \ldots, n-1 \\
\sum_{j=1}^{n} k_{j} \sum_{l \in \mathcal{N}_{i}} w_{i l}\left[\hat{x}_{l i j}(t)-\hat{x}_{i j}(t)\right], t \geq n
\end{array}\right.
$$

where $w_{i l}$ is the edge weight on the communication channel $(l, i)$. 4.14 follows from 9 , 107 where cases of $n=1,2$ were investigated. Some notations are defined as follows:

$$
\begin{aligned}
d(t) & =\left[d_{1}(t), \ldots, d_{N}(t)\right]^{\prime}, & \Delta(t) & =\left[\Delta_{1}(t), \ldots, \Delta_{N}(t)\right]^{\prime}, \\
x_{j}(t) & =\left[x_{1 j}(t), \ldots, x_{N j}(t)\right]^{\prime}, & \hat{x}_{j}(t) & =\left[\hat{x}_{1 j}(t), \ldots, \hat{x}_{N j}(t)\right]^{\prime}, \\
e_{j}(t) & =\left[e_{1 j}(t), \ldots, e_{N j}(t)\right]^{\prime}, & \delta_{j}(t) & =\left[\delta_{1 j}(t), \ldots, \delta_{N j}(t)\right]^{\prime}=\mathbf{J}_{N} x_{j}(t),
\end{aligned}
$$

where $d_{i}$ and $\Delta_{i}$ are defined in Remark 4.4 , and $\mathbf{J}_{N}=I_{N}-\frac{1}{N} \mathbf{1 1}^{\prime}$. We adopt the following widely used assumptions for consensus analysis:

Assumption 4.1. The communication graph $\mathcal{G}$ is undirected and connected.

Assumption 4.2. There exists a known constant $C^{*}$ such that $\max _{j}\left\|x_{j}(0)\right\|_{\infty} \leq C^{*}$.

Remark 4.5. Assumption 4.1 is a standard assumption. If we denote the Laplacian of $\mathcal{G}$ as $L$, then we can find a unitary matrix

$$
T_{L}=\left[\frac{\mathbf{1}_{N}}{\sqrt{N}} \phi_{2} \ldots \phi_{N}\right]
$$


such that $T_{L}^{\prime} L T_{L}=\operatorname{diag}\left\{\lambda_{1}, \lambda_{2}, \ldots, \lambda_{N}\right\}$ and $\phi_{i}$ is the unit eigenvector of $L$ with respect to the eigenvalue $\lambda_{i}$. Moreover, the eigenvalues of $L$ can be rearranged as $0=\lambda_{1}<\lambda_{2} \leq \cdots \leq \lambda_{N}$. Assumption 4.2 enables the quantizer $q_{t}(\cdot)$ to be made unsaturated at initial steps, and we have $\max _{j}\left\|\delta_{j}(0)\right\|_{\infty} \leq 2 C^{*}$.

The rest of this subsection is devoted to the analysis of quantized consensus. Lemmas 4.1 and 4.2 address the simultaneous stabilization and componentwise estimation for the disagreement vector, respectively. Then Remark 4.8 along with Lemma 4.3 shows a specific choice of control gains. Finally, we provide the main result in Theorem 4.1 .

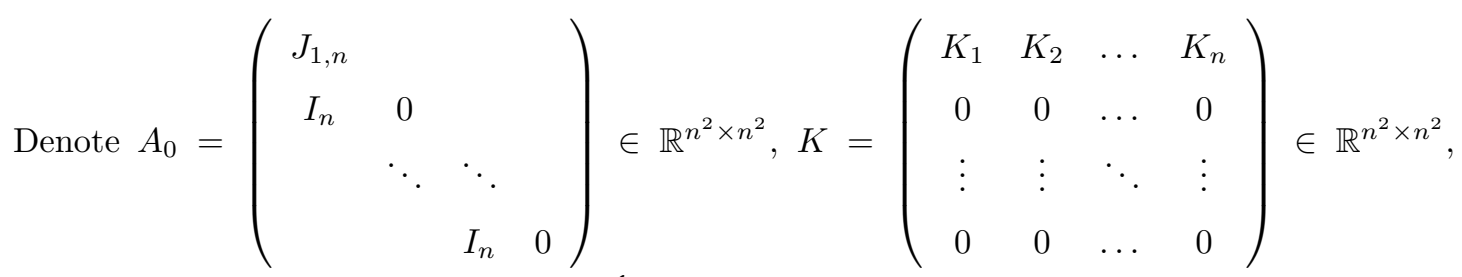

where $K_{j} \in \mathbb{R}^{n \times n}$ is defined by $\left[K_{j}\right]_{m, t}=\left\{\begin{array}{ll}k_{j}, & m=n, t=j ; \\ 0, & \text { otherwise, }\end{array}\right.$ for $j=1, \ldots, n$. The open-loop state matrix $A_{0}$ admits a Jordan chain of length $n$ with respect to the unit eigenvalue, as well as $n$ Jordan chains of length $n-1$ with respect to the zero eigenvalue. For the closed-loop matrices of disagreement dynamics

$$
A_{i}=A_{0}-\lambda_{i} K, i=2, \ldots, N
$$

we first employ standard perturbation techniques [108,109] to solve the stabilization problem, as seen below in Lemma 4.1 whose detailed proof is referred to Section 4.7 .

Lemma 4.1. Assume that $\lambda_{i}>0$. Let

$$
k_{j}= \begin{cases}c_{j} \varepsilon^{n-j}, & j=1,2, \ldots, n-2 \\ c_{j} \varepsilon, & j=n-1, n\end{cases}
$$

and define the equation

$$
c_{n-1} v_{1}^{n-2}+c_{n-2} v_{1}^{n-3}+\cdots+c_{2} v_{1}+c_{1}=0
$$

Then for sufficiently small $\varepsilon$, the spectral radius $\rho_{i}$ of $A_{i}$ can be made less than 1 if the constants $c_{j}$ 's are selected from the set:

$$
\begin{array}{r}
\Omega\left(\lambda_{i}, n\right)=\left\{c_{j}, j=1, \ldots, n \mid 0<c_{n-1}<\frac{c_{n}}{n-1}, \frac{c_{n-2}}{c_{n-1}}<\left(c_{n}-(n-1) c_{n-1}\right) \lambda_{i},\right. \\
4.19 \text { admits } n-2 \text { distinct negative real roots }\} .
\end{array}
$$

In particular, $A_{i}$ has $(n-1)^{2}$ zero eigenvalues, $n-1$ distinct eigenvalues perturbed around 0 , and $n$ 
distinct eigenvalues perturbed around 1 given by

$$
\begin{aligned}
& \chi_{i l}^{(1)}=1+\mu_{i l 1} \varepsilon^{1 / 2}+\mu_{i 2} \varepsilon+o(\varepsilon), l=1,2 ; \\
& \chi_{i l}^{(1)}=1+v_{l 1} \varepsilon+o(\varepsilon), l=3, \ldots, n,
\end{aligned}
$$

where $\mu_{i l 1}=(-1)^{l-1} \sqrt{c_{n-1} \lambda_{i}}$ for $l=1,2, \mu_{i 2}=-\frac{1}{2}\left[\left(c_{n}-(n-2) c_{n-1}\right) \lambda_{i}-\frac{c_{n-2}}{c_{n-1}}\right]$ and $v_{l 1}$ denote the $n-2$ distinct real roots of 4.19.

Remark 4.6. Lemma 4.1 aims to find a common set of control gains to achieve the simultaneous stabilization. Perturbation leads to perturbed eigenvalues expressed in the Newton-Puiseux series shown in 4.21, with the leading coefficient given as the root of a polynomial equation. The tricky part lies in appropriately choosing the power exponents of $\varepsilon$ in $k_{j}$ 's to guarantee an easily solved leading term, and specifically the unknown $\lambda_{i}$ should be not involved in high-order polynomial equations. By using Newton diagram (see the proof in Section 4.7) for different types of $k_{j}$ 's, we found that the choice of $k_{j}$ 's as in 4.18) leads to the result that only two of the $n$ perturbed roots around 1 admit $\lambda_{i}$ in the leading term. This is intuitively possible, as long as we note that the part of the characteristic equation corresponding to the nonzero eigenvalues (see 4.51) in Section 4.7) is equivalent to

$$
\frac{1}{\lambda_{i}} \lambda^{n-1}(\lambda-1)^{n}+k_{n}(\lambda-1)^{n-1}+k_{n-1} \lambda(\lambda-1)^{n-2}+\cdots+k_{1} \lambda^{n-1}=0,
$$

where $\lambda_{i}$ only appears in the first term.

In the case of quantized consensus, the simultaneous stabilization suffices to achieve the convergence result. Still, we need a further analysis on the magnitude of control input to determine the corresponding data rate, which hinges upon the estimation of each component of the disagreement vector as given in the following lemma:

Lemma 4.2. Let the conditions of Lemma 4.1 hold. Then for any vector $\xi \in \mathbb{R}^{n^{2}}$, the first $n$ entries of $A_{i}^{t} \xi, t \in \mathbb{N}^{+}$, denoted as $\xi_{t, j}^{i}, j=1, \ldots, n$, satisfy that

$$
\left|\xi_{t, j}^{i}\right| \leq\left\{\begin{array}{l}
\|\xi\|_{\infty} M_{i j} \rho_{i}^{t} \varepsilon^{j-(n-1)}, j=1,2, \ldots, n-2 \\
\|\xi\|_{\infty} M_{i j} \rho_{i}^{t} \varepsilon^{(j-n) / 2}, j=n-1, n
\end{array}\right.
$$

when $\varepsilon$ is sufficiently small, where

$$
M_{i j}=\left\{\begin{array}{l}
\frac{3}{2 c_{n-1} \lambda_{i}} \sum_{l=3}^{n} \frac{v_{l 1}^{j-1}}{\prod_{s \geq 3, s \neq l}\left|v_{s 1}-v_{l 1}\right|}, j=1, \ldots, n-3 \\
\frac{3}{2 c_{n-1} \lambda_{i}}\left(\sum_{l=3}^{n} \frac{v_{l 1}^{n-3}}{\prod_{s \geq 3, s \neq l}\left|v_{s 1}-v_{l 1}\right|}+1\right), j=n-2 \\
\frac{3}{2 \sqrt{c_{n-1} \lambda_{i}}}, j=n-1 \\
3 / 2, j=n
\end{array}\right.
$$


Remark 4.7. The proof of Lemma 4.2 can be found in Section 4.7. The key is to solve the Jordan decomposition of $A_{i}$, which requires the calculation of the Jordan basis and its inverse. We first find the Jordan basis in a form of Newton-Puiseux series by the techniques in [110] and [111], and then we obtain the first $n$ rows of its inverse by adjoint method. The existence of two different splitting behaviors, namely a complete splitting from a Jordan chain into $n$ different eigenvectors, and a partially preserved Jordan chain plus the splitting eigenvectors, renders the proof much more challenging. It is noted that for the eigenvalue 1 with a Jordan chain of length $n$, the bifurcation of the eigenvalue into $n$ different ones causes a discontinuous change in the Jordan basis, and results in an estimation of $\left\|A_{i}^{t}\right\|_{\infty}=O\left(\varepsilon^{2-n}\right) \rho_{i}^{t}$ when $n \geq 3$. From the later analysis, it is impossible to reduce the magnitude of the control input by letting $\varepsilon$ approach 0 if such an estimation is employed in the data rate analysis. Therefore, we need to conduct a more delicate analysis to reveal the different convergence speeds among different components, as shown in Lemma 4.2. It should also be pointed out that the authors of [9] failed to notice the discontinuity of the Jordan basis and falsely claimed that $\left\|A_{i}^{t}\right\|_{\infty}=O(1) \rho_{i}^{t}$, hence made an inaccurate argument; however, their result of using 2 bits to achieve quantized consensus for the MAS of the second order integrator dynamics still holds as it can be shown that $\left\|A_{i}^{t}\right\|_{\infty}=O\left(\varepsilon^{-1 / 2}\right) \rho_{i}^{t}$ when $k_{1}, k_{2}=O(\varepsilon)$.

The next remark discusses how to choose $c_{j}$ 's and accordingly determine the constants $M_{i j}$ 's.

Remark 4.8. By Lemma 4.1. $\bigcap_{i=2}^{N} \Omega\left(\lambda_{i}, n\right)=\Omega\left(\lambda_{2}, n\right)$ is not empty for $0<\lambda_{2} \leq \cdots \leq \lambda_{N}$. Therefore, we can always select $c_{j}$ 's from $\Omega\left(\lambda_{2}, n\right)$ to simultaneously stabilize $A_{i}$ 's. Moreover, we have

$$
\rho_{i}=1+\max _{l}\left\{-\frac{1}{2}\left[\left(c_{n}-(n-1) c_{n-1}\right) \lambda_{i}-\frac{c_{n-2}}{c_{n-1}}\right], v_{l 1}\right\} \varepsilon+o(\varepsilon)
$$

and

$$
\rho \triangleq \max _{i \geq 2} \rho_{i}=1+\max _{l}\left\{-\frac{1}{2}\left[\left(c_{n}-(n-1) c_{n-1}\right) \lambda_{2}-\frac{c_{n-2}}{c_{n-1}}\right], v_{l 1}\right\} \varepsilon+o(\varepsilon)
$$

As a result, by letting $c_{n-1}=1$, we can always find a sufficiently large $c_{n}$ so that

$$
-\frac{1}{2}\left[\left(c_{n}-(n-1)\right) \lambda_{2}-c_{n-2}\right] \leq \max _{l} v_{l 1}<0
$$

and consequently $\rho=1+\max _{l} v_{l 1} \varepsilon+o(\varepsilon)$. For example, by selecting $c_{j}$ to be the coefficient of term $v_{1}^{j-1}$ in the polynomial $\left(v_{1}+1\right) \ldots\left(v_{1}+n-2\right)$ and $c_{n}=\frac{2+c_{n-2}}{h}+n-1, h \in\left(0, \lambda_{2}\right]$, we obtain 
$\rho=1-\varepsilon+o(\varepsilon)$. Particularly, in this case $M_{i j}$ 's are given by

$$
M_{i j}=\left\{\begin{array}{l}
\frac{3}{2 \lambda_{i}} \sum_{l=1}^{n-2} \frac{l^{j-1}}{(l-1) !(n-2-l) !}, j=1, \ldots, n-2 \\
\frac{3}{2 \lambda_{i}}\left[\sum_{l=1}^{n-2} \frac{l^{n-3}}{(l-1) !(n-2-l) !}+1\right], j=n-2 \\
\frac{3}{2 \sqrt{\lambda_{i}}}, \quad j=n-1 \\
3 / 2, j=n
\end{array}\right.
$$

Based on the above lemmas and Remark 4.8 with $c_{n}>1=c_{n-1}$, we have the following lemma about the choice of $\varepsilon$.

Lemma 4.3. Define $h(t, \gamma)=\left(1+\gamma^{-1}\right)^{t}$. Take $k_{j}$ 's as in 4.18), $c_{j}$ 's as in Remark 4.8, and $\gamma=1-\varepsilon / 4$. Then $\varepsilon$ can be selected to be sufficiently small such that

$$
\begin{aligned}
\max _{j} k_{j}=c_{n} \varepsilon ; \rho & <1-\frac{1}{2} \varepsilon ; \gamma^{3 n-2} \geq 1 / 2, \\
{\left[1+\left(2 d^{*}\right)^{3 / 2}\right] c_{n} \sqrt{\varepsilon} } & \leq 1 /\left(16 N\left(2^{n+1}-1\right)\right), \\
h(t, \gamma) & \leq 2^{t}-1 / 2, t=1, \ldots, n, \\
\left(\sum_{j=1}^{n-2} c_{j} M_{i j}+c_{n} M_{i n}\right) \sqrt{\varepsilon} & \leq \frac{1}{3} M_{i, n-1}, i=2, \ldots, N,
\end{aligned}
$$

where $d^{*}$ is the maximum degree of $\mathcal{G}$ and $M_{i j}$ has been defined in 4.23.

Proof. The proof is straightforward with a sufficiently small $\varepsilon$.

Remark 4.9. For the later application, here we discuss the implication of some of the above conditions. With 4.26), $\gamma^{3 n-2} \geq 1 / 2$ and sufficiently large $p_{0}$ we are able to bound $d(t)$ by $2^{t}+1 / 2$ for $t \leq n$ and the major part of $d(t)$ (ignoring $u(t-n)$ ) by $2^{n}-1 / 4$ for the other $t$. With (4.27) it is able to bound the minor part of $d(t)$, namely $\frac{u(t-n)}{p(t-1)}$ by $O\left(\varepsilon^{1 / 2}\right)$. The discussion about 4.25 is as follows. Noticing that $\lambda_{N} \leq\|L\|_{\infty}=2 d^{*}, \varepsilon \leq \sqrt{\varepsilon}$ and $h(n, \gamma) \leq 2^{n}-1 / 2$, we obtain $\|L\|_{\infty} c_{n} \varepsilon h(n, \gamma) \leq 1 / 32$ and $\|L\|_{\infty} c_{n} \varepsilon\left(2^{n+1}-n-2\right)<1 / 8$. Similarly, by $\left\|T_{L}\right\|_{\infty} \leq \sqrt{N}$ where $T_{L}$ is given by 4.16), we can infer that $8\left\|T_{L}\right\|_{\infty} \sqrt{N} \lambda_{N}^{3 / 2} c_{n} \varepsilon^{1 / 2} h(n, \gamma)<1 / 4$. Moreover, for $\sqrt{\lambda_{N}} \leq 1+\left(2 d^{*}\right)^{3 / 2}$ and $c_{n}>1$, it holds that $2^{n+1}\left\|T_{L}\right\|_{\infty} \sqrt{N} \sqrt{\lambda_{N} \varepsilon}<1 / 4$.

Now we are in a position to present our main result.

Theorem 4.1. Under Assumptions 4.1 and 4.2. take $k_{j}$ 's, $c_{j}$ 's, $\varepsilon$ and $\gamma$ as in Lemma 4.3, and let $p_{0} \geq \frac{16}{3} C^{*}$. Then (4.3) can be achieved with a convergence rate $O\left(\gamma^{t}\right)$ provided that $M(t)$ satisfies

$$
\left\{\begin{array}{l}
M(t) \geq 2^{t-1}, t=1, \ldots, n \\
M(t) \geq 2^{n-1}, t=n+1, \ldots
\end{array}\right.
$$


Moreover, $n$ bits are enough to achieve the consensus.

Proof. The proof will be divided into 3 steps. Firstly, with the control input 4.14 we transform (4.1) into a closed-loop system of disagreements, which is further decoupled into $N-1$ linear subsystems with nonlinear disturbance, plus a subsystem with a constant zero state. Secondly, we apply Lemma 4.1 and Lemma 4.2 to these $N-1$ subsystems, so as to estimate the bounds of the disagreements and show that an exponentially fast consensus can be guaranteed as long as the quantization error is bounded. Finally, we show by induction that a bounded quantization error can be achieved by properly selecting the number of quantization levels, as well as parameters $p_{0}$ and $\varepsilon$. The details of these 3 steps are given as follows.

1). Disagreement dynamics. From 4.10 and 4.14 we have

$$
u(t)=\left\{\begin{array}{l}
0, t=0,1, \ldots, n-1 \\
-\sum_{j=1}^{n} k_{j} L\left(\delta_{j}(t-j+1)+e_{j}(t-j+1)\right), t \geq n .
\end{array}\right.
$$

Direct computation shows that

$$
\left[\begin{array}{c}
\delta_{1}(t+1) \\
\vdots \\
\delta_{n}(t+1)
\end{array}\right]=\left(J_{1, n} \otimes I_{N}\right)\left[\begin{array}{c}
\delta_{1}(t) \\
\vdots \\
\delta_{n}(t)
\end{array}\right]+\left[\begin{array}{c}
0 \\
\vdots \\
u(t)
\end{array}\right]
$$

Let $\tilde{\delta}_{j}(t)=\left[\tilde{\delta}_{1 j}(t), \ldots, \tilde{\delta}_{N j}(t)\right]=T_{L}^{-1} \delta_{j}(t)$ with $T_{L}$ defined in 4.16), and $\tilde{\delta}^{i}(t)=\left[\tilde{\delta}_{i 1}(t), \ldots, \tilde{\delta}_{i n}(t)\right]^{\prime}$. Clearly $\tilde{\delta}^{1}(t) \equiv 0$ due to $\tilde{\delta}_{1 j}(t)=\frac{1}{\sqrt{N}} \mathbf{1}_{N}^{\prime} \mathbf{J}_{N} x_{j}(t)=0$ for any $j$. For $i \geq 2$, it holds that

$$
\begin{aligned}
& \tilde{\delta}^{i}(t+1)=J_{1, n} \tilde{\delta}^{i}(t), t=0,1, \ldots, n-1 ; \\
& \tilde{\delta}^{i}(t+1)=J_{1, n} \tilde{\delta}^{i}(t)+\eta_{i}(t), t \geq n
\end{aligned}
$$

where $\eta_{i}(t)$ is an $n$-dimensional vector with the only non-zero entry of $-\sum_{j=1}^{n} k_{j} \lambda_{i}\left[\tilde{\delta}_{i j}(t-j+1)+\right.$ $\left.\phi_{i}^{\prime} e_{j}(t-j+1)\right]$ on the $n$-th row.

2). Exponentially fast consensus. Obviously, $\tilde{\delta}^{i}(t)=J_{1, n}^{t} \tilde{\delta}^{i}(0), t=1, \ldots, n$. When $t \geq n$, let $z^{i}(t)=\operatorname{col}\left\{\tilde{\delta}^{i}(t), \ldots, \tilde{\delta}^{i}(t-n+1)\right\}$, then imposing 4.30 it leads to that

$$
z^{i}(t+1)=A_{i} z^{i}(t)-\epsilon_{i}(t-n+1)
$$

where $A_{i}$ is defined in 4.17) and $\epsilon_{i}(t-n+1)$ is an $n^{2}$-dimensional vector with the only nonvanishing entry $\sum_{j=1}^{n} k_{j} \lambda_{i} \phi_{i}^{\prime} e_{j}(t-j+1)$ in the $n$-th row. Applying 4.13), we have

$$
\left\|\epsilon_{i}(t-n-k)\right\|_{\infty} \leq \sqrt{N} \lambda_{i} c_{n} \varepsilon p_{0} \gamma^{t-k-1} \sup _{t-n-k \leq \tau \leq t-1-k}\|\Delta(\tau)\|_{\infty} h(n, \gamma)
$$


By 4.22 and 4.32 with $t=n+m, m=1,2, \ldots$, we have

$$
\begin{aligned}
& \quad\left|\tilde{\delta}_{i j}(t)\right|=\left|\tilde{\delta}_{i j}(n+m)\right| \\
& \leq\left\{\begin{array}{l}
M_{i j} \varepsilon^{j-(n-1)}\left(\left\|z^{i}(n)\right\|_{\infty} \rho_{i}^{m}+\sum_{k=0}^{m-1} \rho_{i}^{k}\left\|\epsilon_{i}(t-n-k)\right\|_{\infty}\right), j=1, \ldots, n-2 ; \\
M_{i j} \varepsilon^{(j-n) / 2}\left(\left\|z^{i}(n)\right\|_{\infty} \rho_{i}^{m}+\sum_{k=0}^{m-1} \rho_{i}^{k}\left\|\epsilon_{i}(t-n-k)\right\|_{\infty}\right), j=n-1, n .
\end{array}\right. \\
& \leq\left\{\begin{array}{l}
M_{i j} \varepsilon^{j-(n-1)}\left(\left\|z^{i}(n)\right\|_{\infty} \rho_{i}^{m}+\sqrt{N} \lambda_{i} c_{n} \varepsilon p_{0} \gamma^{t-1} \sup _{1 \leq \tau \leq t-1}\|\Delta(\tau)\|_{\infty} h(n, \gamma) \frac{\gamma}{\gamma-\rho}\right), \\
j=1, \ldots, n-2 ; \\
M_{i j} \varepsilon^{(j-n) / 2}\left(\left\|z^{i}(n)\right\|_{\infty} \rho_{i}^{m}+\sqrt{N} \lambda_{i} c_{n} \varepsilon p_{0} \gamma^{t-1} \sup _{1 \leq \tau \leq t-1}\|\Delta(\tau)\|_{\infty} h(n, \gamma) \frac{\gamma}{\gamma-\rho}\right), \\
j=n-1, n .
\end{array}\right.
\end{aligned}
$$

Moreover, we can see that

$$
\left\|z^{i}(t)\right\|_{\infty}=\left\|J^{(t)} \tilde{\delta}^{i}(0)\right\| \leq 2^{t} \sqrt{N} \max _{j}\left\|\delta_{j}(0)\right\|_{\infty}, t=1, \ldots, n
$$

with $J^{(t)}=\left[\left(J_{1, n}^{t}\right)^{\prime}\left(J_{1, n}^{t-1}\right)^{\prime} \ldots\left(J_{1, n}\right)^{\prime}\right]^{\prime}$, because $\left\|J^{(t)}\right\|_{\infty} \leq 2^{t}$ and $\left|\phi_{i}^{\prime} \delta_{j}(0)\right| \leq \sqrt{N}\left\|\delta_{j}(0)\right\|_{\infty}$. From 4.34 and the definition of $\tilde{\delta}^{i}(t)$, the consensus can be achieved with the convergence rate $O\left(\gamma^{t}\right)$ once $\sup _{1 \leq \tau \leq t-1}\|\Delta(\tau)\|_{\infty}$ is bounded.

3). Data rate. In what follows, we show $\|\Delta(t)\|_{\infty} \leq \frac{1}{2}$ by induction. Recalling 4.11 and 4.13, it follows that for $t=1,2, \ldots, n$,

$$
\|d(t)\|_{\infty} \leq \frac{1}{2} h(t, \gamma)-\frac{1}{\gamma^{t}}+\frac{2 C^{*}}{p_{0} \gamma^{t-1}} \leq 2^{t-1}+1 / 2
$$

and for $t=n+1, \ldots, 2 n-1$,

$$
\|d(t)\|_{\infty} \leq \frac{1}{2} h(n, \gamma) \leq 2^{n-1}-1 / 4
$$

if we note that 4.26,,$p_{0} \geq \frac{16}{3} C^{*}$ and $\gamma^{t-1}>\gamma^{3 n-2} \geq 1 / 2$ when $t=1, \ldots, n$. 
Moreover, if $\sup _{1 \leq \tau \leq t-1}\|\Delta(\tau)\|_{\infty} \leq 1 / 2$, then by 4.34) and (4.27), when $t \geq 3 n$,

$$
\begin{aligned}
& \left\|\sum_{j=1}^{n} k_{j} L\left(\delta_{j}(t-n-j+1)\right)\right\|_{\infty} \\
\leq & \left\|T_{L}\right\|_{\infty} \max _{i}\left|\sum_{j=1}^{n} k_{j} \lambda_{i} \tilde{\delta}_{i j}(t-n-j+1)\right| \\
\leq & \left\|T_{L}\right\|_{\infty} \max _{i} \lambda_{i}\left[\left\|z^{i}(n)\right\|_{\infty}\left(\sum_{j=1}^{n-2} c_{j} \varepsilon M_{i j} \rho_{i}^{t-2 n-j+1}+c_{n-1} \varepsilon^{1 / 2} M_{i, n-1} \rho_{i}^{t-3 n+2}+c_{n} \varepsilon M_{i n} \rho_{i}^{t-3 n+1}\right)\right. \\
& +\sqrt{N} \lambda_{i} c_{n} \varepsilon \sup _{1 \leq \tau \leq t-1}\|\Delta(\tau)\|_{\infty} h(n, \gamma) \frac{p_{0} \gamma}{\gamma-\rho} \cdot \\
& \left.\left(\sum_{j=1}^{n-2} c_{j} \varepsilon M_{i j} \gamma^{t-n-j}+c_{n-1} \varepsilon^{1 / 2} M_{i, n-1} \gamma^{t-2 n+1}+c_{n} \varepsilon M_{i n} \gamma^{t-2 n}\right)\right] \\
\leq & \left\|T_{L}\right\|_{\infty}\left[2\left\|z^{i}(n)\right\|_{\infty} \sqrt{\lambda_{N} \varepsilon} \rho^{t-3 n+1}+4 \sqrt{N} \lambda_{N}^{3 / 2} c_{n} \varepsilon^{1 / 2} h(n, \gamma) p_{0} \gamma^{t-2 n}\right]
\end{aligned}
$$

similarly, when $2 n \leq t \leq 3 n-1$,

$$
\begin{aligned}
& \left\|\sum_{j=1}^{n} k_{j} L \delta_{j}(t-n-j+1)\right\|_{\infty} \\
\leq & \left\|\sum_{j=1}^{t-2 n} k_{j} L \delta_{j}(t-n-j+1)\right\|_{\infty}+\left\|\sum_{j=t-2 n+1}^{n} k_{j} L \delta_{j}(t-n-j+1)\right\|_{\infty} \\
\leq & \left\|T_{L}\right\|_{\infty}\left[2\left\|z^{i}(n)\right\|_{\infty} \sqrt{\lambda_{N} \varepsilon} \rho+4 \sqrt{N} \lambda_{N}^{3 / 2} c_{n} \varepsilon^{1 / 2} h(n, \gamma) p_{0} \gamma^{n}\right] \\
& +\|L\|_{\infty} c_{n} \varepsilon\left(2^{n+1}-n-2\right) \max _{j}\left\|\delta_{j}(0)\right\|_{\infty} .
\end{aligned}
$$

Note that

$$
\begin{aligned}
\|d(t)\|_{\infty}= & \frac{1}{p(t-1)}\left\|-\sum_{j=1}^{n} e_{j}(t-j)+u(t-n)\right\|_{\infty} \\
\leq & \frac{1}{2} h(n, \gamma)+\frac{\|L\|_{\infty}}{p(t-1)}\left\|\sum_{j=1}^{n} k_{j} e_{j}(t-n-j+1)\right\|_{\infty} \\
& +\frac{1}{p(t-1)}\left\|\sum_{j=1}^{n} k_{j} L \delta_{j}(t-n-j+1)\right\|_{\infty} \\
\leq & \frac{1}{2} h(n, \gamma)+\frac{\|L\|}{2 \gamma^{n-1}} c_{n} \varepsilon h(n, \gamma)+\frac{1}{p(t-1)}\left\|\sum_{j=1}^{n} k_{j} L \delta_{j}(t-n-j+1)\right\|_{\infty}
\end{aligned}
$$

which, together with 4.35 and 4.36 , yields that

$$
\|d(t)\|_{\infty} \leq 2^{n-1}+1 / 2, t \geq 3 n
$$


as a result by combining 4.24, $p_{0} \geq 2 C^{*} \geq \max _{j}\left\|\delta_{j}(0)\right\|_{\infty}$ and the discussion of 4.25 in Remark 4.9 Hence, $\|\Delta(t)\|_{\infty} \leq 1 / 2$ when $M(t)$ is taken as in 4.28). In view of Remark 4.1. $n$ bits are enough to realize the leaderless consensus.

Remark 4.10. The conditions 4.26) and 4.27) can be replaced by equivalent or stronger ones, which are more convenient to check. For 4.26), by direct computation we have $\left(2^{t}-1 / 2\right)^{t-1} \leq\left(2^{t-1}-1 / 2\right)^{t}$, suggesting that

$$
h(t, \gamma) \leq 2^{t}-1 / 2 \Rightarrow h(t-1, \gamma) \leq 2^{t-1}-1 / 2
$$

Hence it is equivalent to

$$
h(n, \gamma) \leq 2^{n}-1 / 2
$$

For 4.27), noticing that $(l-1) !(n-2-l) !$ attains the minimum at $l=\left\lfloor\frac{n-2}{2}\right\rfloor$, it can be substituted by the following:

$$
\left(\sum_{j=1}^{n-2} c_{j} \sum_{l=1}^{n-2} \frac{l^{j-1}}{\left(\left\lfloor\frac{n}{2}\right\rfloor-2\right) !\left(n-1-\left\lfloor\frac{n}{2}\right\rfloor\right) !}+c_{n-2}+2 c_{n} d^{*}\right) \sqrt{\varepsilon} \leq \frac{\sqrt{\lambda_{2}}}{3}
$$

Summing up the discussion above gives rise to the following corollary.

Corollary 4.1. Take $k_{j}$ 's as in (4.18), $c_{j}$ 's as in Remark 4.8, and $\gamma=1-\varepsilon / 4$. Under Assumptions 4.1 and 4.2. if $p_{0} \geq \frac{16}{3} C^{*}$ and $\varepsilon$ is selected to satisfy 4.24, 4.25, 4.37) and 4.38, then (4.3) can be achieved with a convergence rate $O\left(\gamma^{t}\right)$ provided that $M(t)$ satisfies (4.28). Moreover, $n$ bits are enough to achieve the consensus.

Remark 4.11. In this work we mainly focus on finding the possibly lowest data rate for quantized consensus. From 4.34 it can be seen that $\gamma$ can only be selected from $(\rho, 1)$ to guarantee the quantized consensus under a limited data rate, and hence slow down the convergence rate when compared with the case of perfect information. As demonstrated in [107] for the first order dynamics, a lower data rate implies a slower convergence. It is of interest and importance to analyze the optimal convergence rate under a given data rate, but the designing of control gains is much more challenging for higher-order dynamics, which will be our future work.

Remark 4.12. From the proof we can see that the number of bits required for exchanging data between agents is dependent on the magnitude of $d(t)$, which consists of two scaled terms: $\frac{-1}{p(t-1)} \sum_{j=1}^{n} e_{j}(t-j)$ and $\frac{1}{p(t-1)} u(t-n)$. For the first part, by 4.13 it can be bounded by $2^{n-1}-\frac{1}{4}$ as long as the quantizer is saturated in previous time instants, and $\gamma$ close to 1 ; for the second part, $u(t-n)=O\left(\varepsilon^{1 / 2}\right) p_{0} \gamma^{t-3 n+1}$, due to the fact that the $\varepsilon$ term in control gain $k_{j}$ always has a larger exponent than that of the $\varepsilon^{-1}$ term in the $j$-th disagreement vector $\delta_{j}(t)$. Hence the scaled control input, i.e. the second term, can be reduced as much as possible with sufficiently small $\varepsilon$. In other words, the control input does not 
consume any bit in exchanging the estimates of the system states with neighboring agents. However, the above argument is not satisfied generally when the system has eigenvalues outside the unit circle, because under such a circumstance perturbation techniques are not eligible for solving the stabilization problem.

\subsubsection{Leader-following Consensus}

Consider an MAS with a single leader and let agent 0 be the leader. The distributed leader-following consensus control law is proposed as

$$
u_{i}(t)=\left\{\begin{array}{l}
0, t=0,1, \ldots, n-1 \\
\sum_{j=1}^{n} k_{j}\left\{\sum_{l \in \mathcal{N}_{i}} w_{i l}\left[\hat{x}_{l i j}(t)-\hat{x}_{i j}(t)\right]+b_{i}\left[\hat{x}_{0 i j}(t)-\hat{x}_{i j}(t)\right]\right\}, t \geq n,
\end{array}\right.
$$

where $w_{i l}$ is the edge weight on the communication channel $(l, i)$ between followers $i$ and $j$, and $b_{i} \geq 0$ is the connection weight between agent $i$ and the leader. Note that $b_{i}>0$ iff agent $i$ is connected to the leader. Let $\mathcal{G}=\{\mathcal{N}, \mathcal{E}\}$ be the communication graph of followers, and denote $\overline{\mathcal{G}}=\{\overline{\mathcal{N}}, \overline{\mathcal{E}}\}$ as the whole communication topology with $\overline{\mathcal{N}}=\mathcal{N} \cup\{0\}, \overline{\mathcal{E}}=\mathcal{E} \cup\left\{(0, i): b_{i}>0, i=1, \ldots, N\right\}$. With some abuse, the notations in 4.15 still apply with $\delta_{i j}(t)$ replaced by $\delta_{i j}(t)=x_{i j}(t)-x_{0 j}(t)$.

We adopt the following assumptions:

Assumption 4.3. The communication graph $\overline{\mathcal{G}}$ contains a spanning tree rooted at the leader 0 , and $\mathcal{G}$ is undirected.

Assumption 4.4. There exist known positive constants $C^{*}$ and $C_{l}^{*}$ such that $\max _{j}\left\|x_{j}(0)\right\|_{\infty} \leq C^{*}$ and $\max _{j}\left|x_{0 j}(0)\right| \leq C_{l}^{*}$.

Remark 4.13. Define $\bar{L}=L+B$, where $L$ is the Laplacian of $\mathcal{G}$ and $B=\operatorname{diag}\left\{b_{1}, \ldots, b_{N}\right\}$. Then $\bar{L}$ is positive definite [112], and we can find a unitary matrix

$$
T_{\bar{L}}=\left[\bar{\phi}_{1}, \bar{\phi}_{2}, \ldots, \bar{\phi}_{N}\right]
$$

such that $T_{\bar{L}}^{\prime} \bar{L} T_{\bar{L}}=\operatorname{diag}\left\{\bar{\lambda}_{1}, \ldots, \bar{\lambda}_{N}\right\}$ with $0<\bar{\lambda}_{1} \leq \cdots \leq \bar{\lambda}_{N}$. Under Assumption 4.3 we have $\max _{j}\left\|\delta_{j}(0)\right\|_{\infty} \leq C^{*}+C_{l}^{*}$.

A similar result holds for the leader-following consensus:

Theorem 4.2. Take $k_{j}$ 's as in $(4.18), c_{j}$ 's as in Remark 4.8 with $\lambda_{2}$ replaced by $\bar{\lambda}_{1}$, and $\gamma=1-\varepsilon / 4$. Denote $\bar{d}^{*}=\max _{i}\left\{b_{i}+2 d_{i}\right\}$, where $d_{i}$ is the degree of node $i$ in $\mathcal{G}$. Under Assumptions 4.3 and 4.4 . if $p_{0} \geq \frac{16}{3}\left(C^{*}+C_{l}^{*}\right)$ and $\varepsilon$ satisfies 4.24, 4.37), 4.38) with $2 d^{*}$ replaced by $\bar{d}^{*}$, as well as

$$
\left[1+\left(\bar{d}^{*}\right)^{3 / 2}\right] c_{n} \varepsilon^{1 / 2} \leq 1 /\left(32\left(2^{n+1}-1\right)\right)
$$


then (4.4) can be achieved with convergence rate $O\left(\gamma^{t}\right)$ in the light of (4.28). Furthermore, $n$ bits are enough to achieve the leader-following consensus.

Proof. From 4.10 and 4.39, we have

$$
u(t)=\left\{\begin{array}{l}
0, t=0,1, \ldots, n-1 \\
-\sum_{j=1}^{n} k_{j}\left[\bar{L}\left(\delta_{j}(t-j+1)+e_{j}(t-j+1)\right)-\mathbf{1}_{N} \otimes b_{j} e_{0 j}(t-j+1)\right], t \geq n .
\end{array}\right.
$$

Clearly, we get a same expression as 4.30. Let $\tilde{\delta}_{j}(t)=T_{\bar{L}}^{-1} \delta_{j}(t)$, then we obtain an expression of $\tilde{\delta}_{i j}$ similar to 4.31, where $\lambda_{i}, \phi_{i}$ and $e_{j}(t-j+1)$ are replaced by the corresponding terms. The conclusion can be reached by proceeding along the same line of arguments as in the proof of Theorem 4.1

Remark 4.14. It is noted that in both the leaderless and leader-following cases we require an undirected communication graph to guarantee a diagonalizable Laplacian. For general directed graphs whose Laplacian may contain complex eigenvalues or a Jordan block of dimension greater than 1, although the perturbation method can still be employed to achieve the quantized consensus, the corresponding data rate may be far more than $n$. Particularly we illustrate the latter case with a leader-following example as below. Assume a 4-agent system with double-integrator dynamics connected as $0 \rightarrow 1 \rightarrow 2 \rightarrow 3$, with the weight 1 on each edge and the agent 0 as leader. Obviously, $\bar{L}$ consists of a Jordan block $J_{1,3}$ and by the control law (4.39) we can find the following:

$$
\begin{aligned}
& z^{1}(t+1)=A_{2} z^{1}(t)-\epsilon_{1}(t-1), \\
& z^{2}(t+1)=A_{2} z^{2}(t)+K z^{1}(t)-\epsilon_{2}(t-1), \\
& z^{3}(t+1)=A_{2} z^{3}(t)+K z^{2}(t)-\epsilon_{3}(t-1),
\end{aligned}
$$

where $z^{i}(t)=\operatorname{col}\left\{\delta^{i}(t), \delta^{i}(t-1)\right\}$ with $\delta^{i}(t)=x^{i}(t)-x^{0}(t)$, and $A_{2}=A_{0}-K$ with $K=\left[\begin{array}{cccc}0 & 0 & 0 & 0 \\ k_{1} & 0 & 0 & k_{2} \\ 0 & 0 & 0 & 0 \\ 0 & 0 & 0 & 0\end{array}\right]$. Let $k_{1}=c_{1} \varepsilon, k_{2}=c_{2} \varepsilon$ and we get $\epsilon_{i}(t)=O(\varepsilon) p_{0} \gamma^{t}$ due to the presence of quantization error. Then we obtain $z^{1}(t)=O\left(\varepsilon^{-1 / 2}\right) p_{0} \gamma^{t}$ and $K z^{1}(t)=O\left(\varepsilon^{1 / 2}\right) p_{0} \gamma^{t}$; in consequence, $z^{2}(t)=O\left(\varepsilon^{-1}\right) p_{0} \gamma^{t}$ and $z^{3}(t)=O\left(\varepsilon^{-3 / 2}\right) p_{0} \gamma^{t}$, leading to $u_{3}(t)=O\left(\varepsilon^{-1 / 2}\right) p_{0} \gamma^{t}$. In this case the number of quantization levels for agent 3 to maintain an unsaturated quantizer will go to infinity when $\varepsilon$ tends to 0. Moreover, for the above weighted graph, it can be shown with the help of Newton diagram (see the proof in Section $4.7)$ that for any choice of the exponents of $\varepsilon$ in $k_{1}, k_{2}$, it holds that $u_{3}(t)=O\left(\varepsilon^{-\beta}\right) p_{0} \gamma^{t}, \beta>0$. In the case of a diagonalizable $\bar{L}$ (e.g. by selecting distinct weights as 1, 2, 3), 2 bits of data exchange still guarantee the consensus. Different from the case discussed in Remark 4.12, the existence of the Jordan 
block leads to an increased exponent of $\varepsilon^{-1}$ in the disagreement vector, and extra bits are needed to account for the control input. Therefore, we have to seek for other methods to reduce the data rate in handling the case of general directed graphs.

Remark 4.15. Under a connected network, both leaderless and leader-following consensus can be achieved with $n$ bits of communication cost by properly assigning the scaling function for the control gains. This result is consistent with those for the single and double integrator cases, but the analysis is much more challenging and the method in [8, 9] is not applicable. Two crucial steps are required to establish the result: stabilization analysis, as well as the minimization of the magnitude of the scaled control input. We exploit perturbation techniques to tackle both problems. In particular, for the second problem, it needs a much more accurate estimation about the convergence of each component in the disagreement vector, which is obtained by an explicit calculation of Jordan decomposition of perturbed matrices. Though all the analysis in this section is predicated upon the special structure of integrator dynamics, we shall see in the next section that similar results hold for general systems when we apply the same procedure to their observer system.

\subsection{Quantized Consensus and Data Rate for General Systems with Unity Eigenvalues}

In this section we are to investigate a general case when each agent takes the following form:

$$
\left\{\begin{aligned}
x^{i}(t+1) & =A x^{i}(t)+b u_{i}(t), \\
y_{i}(t) & =c x^{i}(t)
\end{aligned}\right.
$$

where $x^{i}(t)=\left[x_{i 1}(t), \ldots, x_{i n}(t)\right]^{\prime} \in \mathbb{R}^{n}, y_{i}(t), u_{i}(t) \in \mathbb{R}$, represent the state, output and input of agent $i$ for $i=0,1, \ldots, N$, respectively. Moreover, $b, c^{\prime} \in \mathbb{R}^{n}$, and $A \in \mathbb{R}^{n \times n}$ only admits identical eigenvalues of 1 . $(A, b)$ and $(c, A)$ are controllable and observable pairs, respectively.

Without loss of generality we assume that the communication topology is identical to that in the last section, and Assumptions 4.1, 4.2, and Assumptions 4.3, 4.4 still hold respectively for the leaderless and leader-following cases. In the sequel, we mainly focus on the leaderless case. The leader-following case can be handled similarly.

To employ the result obtained in the last section, we first show that the controllable pair $(A, b)$ is equivalent to $\left(A^{*}, b^{*}\right)$, where $A^{*}$ and $b^{*}$ correspond to the special case studied in the last section. Actually, $A$ and $A^{*}$ have the same characteristic polynomial, hence $(A, b)$ and $\left(A^{*}, b^{*}\right)$ correspond to the same canonical controller form $\left(A_{c}, b_{c}\right)$. In consequence, there exists a nonsingular $P$ such that 
$A^{*}=P^{-1} A P, b=P^{-1} b^{*}$. Letting $\chi^{i}(t)=P^{-1} x^{i}(t)$, the original system now becomes

$$
\left\{\begin{aligned}
\chi^{i}(t+1) & =A^{*} \chi^{i}(t)+b^{*} u_{i}(t) \\
y_{i}(t) & =\tilde{c} \chi^{i}(t)
\end{aligned}\right.
$$

where $\tilde{c}=c P$. To solve the quantized consensus problem of 4.43 , the following observer is built for every agent:

$$
\left\{\begin{aligned}
\tilde{\chi}^{i}(t+1) & =A^{*} \tilde{\chi}^{i}(t)+b^{*} u_{i}(t)+F\left(y_{i}(t)-\tilde{c} \tilde{\chi}^{i}(t)\right), \\
\tilde{\chi}^{i}(0) & =0
\end{aligned}\right.
$$

where $\tilde{\chi}^{i}(t)=\left[\tilde{\chi}_{i 1}(t), \ldots, \tilde{\chi}_{i n}(t)\right]^{\prime}$. The estimation error of the observer is given by

$$
\epsilon^{i}(t)=\tilde{\chi}^{i}(t)-\chi^{i}(t)=\left(A^{*}-F \tilde{c}\right) \epsilon^{i}(t-1)
$$

Noting that observability is preserved under similarity transformation, we can find a proper $F$ such that $\rho\left(A^{*}-F \tilde{c}\right)=\beta<1$, and $\left\|\epsilon^{i}(t)\right\|_{\infty}=O\left(\beta^{t}\right)$. Like 4.8-4.9, we can respectively construct the encoder and decoder for the observer system 4.44 of agent $i$, and its neighbor agent $l$ by replacing $\hat{x}, y_{i}, s_{i}$ respectively with $\hat{\chi}, \tilde{\chi}_{i 1}, \tilde{s}_{i}$.

Denote

$$
\tilde{d}_{i}(t)=\left\{\begin{array}{l}
\frac{\tilde{\chi}_{i 1}(t)-\sum_{j=1}^{t} \hat{\chi}_{i j}(t-1)}{p(t-1)}, t=1,2, \ldots, n-1 ; \\
\frac{\tilde{\chi}_{i 1}(t)-\sum_{j=1}^{n} \hat{\chi}_{i j}(t-1)}{p(t-1)}, t \geq n
\end{array}\right.
$$

$\tilde{\Delta}_{i}(t)=\tilde{s}_{i}(t)-\tilde{d}_{i}(t)$ as the quantization error, and $\tilde{e}_{i j}(t-j+1)=\hat{\chi}_{i j}(t)-\tilde{\chi}_{i j}(t-j+1)$ as the estimation error for $\tilde{\chi}_{i j}(t-j+1)$. Then

$$
\left\{\begin{aligned}
\tilde{e}_{i 1}(t) & =p(t-1) \Delta_{i}(t), t \geq 1 \\
\tilde{e}_{i 2}(t-1) & =\tilde{e}_{i 1}(t)-\tilde{e}_{i 1}(t-1)-\Theta(1, \cdot) \epsilon^{i}(t-1) \\
& \vdots \\
\tilde{e}_{i n}(t-n+1) & =\tilde{e}_{i, n-1}(t-n+2)-\tilde{e}_{i, n-1}(t-n+1) \\
& -\Theta(n-1, \cdot) \epsilon^{i}(t-n+1)
\end{aligned}\right.
$$

where $\Theta(1, \cdot), \ldots, \Theta(n-1, \cdot)$ are the corresponding rows of $\Theta=F \tilde{c}$. It follows that

$$
\begin{aligned}
\sum_{j=1}^{\tau} \tilde{e}_{i j}(t-j+1)= & \sum_{j=1}^{\tau} C(\tau, j)(-1)^{j-1} \tilde{e}_{i 1}(t-j+1) \\
& +\sum_{k=1}^{\tau-1} \sum_{j=1}^{\tau-k} C(\tau-k, j)(-1)^{j} \Theta(k, \cdot) \epsilon^{i}(t-k), \tau=1,2, \ldots, n .
\end{aligned}
$$

Remark 4.16. Some may wonder why we choose to provide the encoding-decoding scheme for the 
observer system instead of the original system. In fact, this is based upon two considerations. First, recalling Remark 4.3. the special structure of (4.1) allows the design of (4.8)-(4.9) to reduce the number of quantization levels, while in the general case it is difficult to make an iterative estimation of the state as in 4.5). Second, even if we can find a feasible encoding-decoding scheme for the original system, the general input matrix $b$ gives rise to the coupling of control gains and greatly complicates the analysis. Therefore, we construct our encoding-decoding scheme based on the observer system.

Similar to 4.14, the control input $u(t)=\left[u_{1}(t), \ldots, u_{N}(t)\right]^{\prime}$ is given by

$$
u_{i}(t)=\left\{\begin{array}{l}
0, t=0,1, \ldots, n-1 \\
\sum_{j=1}^{n} k_{j} \sum_{l \in \mathcal{N}_{i}} w_{i l}\left[\hat{\chi}_{l i j}(t)-\hat{\chi}_{i j}(t)\right], t \geq n .
\end{array}\right.
$$

Denote $\epsilon_{j}(t)=\left[\epsilon_{1 j}(t), \ldots, \epsilon_{N j}(t)\right]^{\prime}, \tilde{\chi}_{j}(t)=\left[\tilde{\chi}_{1 j}(t), \ldots, \tilde{\chi}_{N j}(t)\right]^{\prime}$ and $\tilde{\delta}_{j}(t)=\mathbf{J}_{N} \tilde{\chi}_{j}(t)$. Clearly the quantized consensus is achieved if and only if $\tilde{\delta}_{j}(t) \rightarrow 0, t \rightarrow \infty$. The dynamics of $\tilde{\delta}_{j}(t)$ can be found as

$$
\left[\begin{array}{c}
\tilde{\delta}_{1}(t+1) \\
\vdots \\
\tilde{\delta}_{n}(t+1)
\end{array}\right]=\left(J_{1, n} \otimes I_{N}\right)\left[\begin{array}{c}
\tilde{\delta}_{1}(t) \\
\vdots \\
\tilde{\delta}_{n}(t)
\end{array}\right]+\left[\begin{array}{c}
0 \\
\vdots \\
u(t)
\end{array}\right]-\left[\Theta \otimes \mathbf{J}_{N}\right]\left[\begin{array}{c}
\epsilon_{1}(t) \\
\vdots \\
\epsilon_{n}(t)
\end{array}\right] .
$$

Moreover, when $t<n$,

$$
\left\|\tilde{d}_{i}(t)\right\|_{\infty}=\frac{1}{p(t-1)}\left\|-\sum_{j=1}^{t} \tilde{e}_{i j}(t-j)-\sum_{j=1}^{t} \Theta(j, \cdot) \epsilon^{i}(t-j)\right\|_{\infty}
$$

when $t \geq n$

$$
\left\|\tilde{d}_{i}(t)\right\|_{\infty}=\frac{1}{p(t-1)}\left\|-\sum_{j=1}^{n} \tilde{e}_{i j}(t-j)-\sum_{j=1}^{n} \Theta(j, \cdot) \epsilon^{i}(t-j)+u_{i}(t-n)\right\|_{\infty} .
$$

Noticing that $\epsilon_{j}(t)=O\left(\beta^{t}\right)$ with arbitrary $\beta$, it can be similarly shown that $n$ bits suffice to achieve the quantized consensus.

Assume that $\left\|\left(A^{*}-F \tilde{c}\right)^{t}\right\|_{\infty} \leq \sigma \beta^{t}$ for $t \geq 0$, and $\beta=\alpha \gamma, 0<\alpha<1$. The discussion above can be summed up as below:

Theorem 4.3. Take $k_{j}$ 's as in 4.18), $c_{j}$ 's as in Remark 4.8, and $\gamma=1-\varepsilon / 4$. Select a sufficiently small $\varepsilon$ to satisfy 4.24, 4.37, 4.38 and

$$
\left[1+\left(2 d^{*}\right)^{3 / 2}\right] c_{n} \varepsilon^{1 / 2} \leq 1 /\left(16 N \cdot 2^{n+1}\right) .
$$


Moreover, let $\alpha^{n} \leq 2 c_{n} \varepsilon d^{*}$. Under Assumptions 4.1 and 4.2, if

$$
p_{0} \geq \max \left\{\frac{16}{3}\left[m_{1}(n, \beta)+\frac{1-\beta^{n}}{1-\beta}\right], \sum_{t=1}^{n} \beta^{t} m_{2}(t, \beta)\right\} \cdot \sigma\|\Theta\|_{\infty}\left\|P^{-1}\right\|_{\infty} C^{*},
$$

where $m_{1}(n, \beta)=\sum_{k=1}^{n-1}\left(2^{n-k}-1\right) \beta^{-k}$ and $m_{2}(t, \beta)=\sum_{\tau=0}^{t-1}(2 / \beta)^{\tau}$, then 4.3 can be achieved with convergence rate $O\left(\gamma^{t}\right)$ in the light of 4.28 and $n$ bits are enough to achieve the consensus.

Similarly, for the leader-following case, we have

Theorem 4.4. Take $k_{j}$ 's as in (4.18), $c_{j}$ 's as in Remark 4.8 with $\lambda_{2}$ replaced by $\bar{\lambda}_{1}$, and $\gamma=1-\varepsilon / 4$. Select a sufficiently small $\varepsilon$ to satisfy 4.24, 4.37), 4.38) with $2 d^{*}$ replaced by $\bar{d}^{*}$ and

$$
\left[1+\left(\bar{d}^{*}\right)^{3 / 2}\right] c_{n} \varepsilon^{1 / 2} \leq 1 /\left(32 N \cdot 2^{n+1}\right)
$$

Moreover, let $\alpha^{n} \leq c_{n} \varepsilon \bar{d}^{*}$. Under Assumptions 4.3 and 4.4. if

$$
p_{0} \geq \max \left\{\frac{16}{3}\left[m_{1}(n, \beta)+\frac{1-\beta^{n}}{1-\beta}\right], \sum_{t=1}^{n} \beta^{t} m_{2}(t, \beta)\right\} \cdot \sigma\|\Theta\|_{\infty}\left\|P^{-1}\right\|_{\infty} \max \left\{C^{*}, C_{l}^{*}\right\}
$$

then (4.4) can be achieved with convergence rate $O\left(\gamma^{t}\right)$ in the light of (4.28) and $n$ bits are enough to achieve the consensus.

Remark 4.17. Comparing the above two theorems with their counterparts in section 4.2, we can see that $p_{0}$ is independent of the constant $C_{\delta}^{*}$, due to the identical zero initial value in the state observers of all the agents.

\subsection{Consensus Value and Application}

In this section we shall study the consensus value and discuss the application of our results to formation control. For the first part, due to the zero-sum of control inputs among different agents, the consensus value can be found in the following theorem.

Theorem 4.5. For the MAS 4.42, if the quantized consensus is achieved under control protocol 4.45, then $\lim _{t \rightarrow \infty} x^{i}(t)=A^{t} \bar{x}(0)$, where $\bar{x}(0)=\frac{1}{N} \sum_{i=1}^{N} x^{i}(0)$.

Proof. We only need to consider the system 4.43) after the similarity transformation. By 4.45 it is easy to check that $\sum_{i=1}^{N} u_{i}(t) \equiv 0$ and consequently the average state

$$
\bar{x}(t)=\frac{1}{N} \sum_{i=1}^{N} w^{i}(t)=A^{*} \bar{x}(t-1)=\left(A^{*}\right)^{t} \bar{x}(0)
$$


where $\bar{x}(0)=P^{-1} \bar{x}(0)$. By quantized consensus, we have $\lim _{t \rightarrow \infty} w^{i}(t)=\bar{x}(t)$ and $\lim _{t \rightarrow \infty} x^{i}(t)=$ $P \bar{x}(t)=P\left(A^{*}\right)^{t} \bar{x}(0)=A^{t} \bar{x}(0)$.

Now we shall employ the results of quantized consensus to study the formation control problem. For the MAS 4.42 and a set of formation vectors $\left\{r^{1}, \ldots, r^{N}\right\}, 4.42$ is said to be formationable if there exists a distributed control protocol such that

$$
\lim _{t \rightarrow \infty}\left[\left(x^{i}(t)-r^{i}\right)-\left(x^{j}(t)-r^{j}\right)\right]=0, \forall i, j .
$$

Consider the transformed system 4.43 and denote $\tilde{r}^{i}=\left[\tilde{r}_{i 1}, \ldots, \tilde{r}_{i n}\right]^{\prime}=P^{-1} r^{i}, \tilde{r}_{j}=\left[\tilde{r}_{1 j}, \ldots, \tilde{r}_{n j}\right]^{\prime}$. In the context of formation control, the quantized control protocol 4.45 would be adapted to

$$
u_{i}(t)=\left\{\begin{array}{l}
0, t=0,1, \ldots, n-1 \\
\sum_{j=1}^{n} k_{j} \sum_{l \in \mathcal{N}_{i}} w_{i l}\left[\left(\hat{\chi}_{l i j}(t)-\tilde{r}_{l j}\right)-\left(\hat{\chi}_{i j}(t)-\tilde{r}_{i j}\right)\right], t \geq n .
\end{array}\right.
$$

Obviously, 4.43 is formationable if and only if $\delta_{j}(t)=\mathbf{J}_{N}\left(\chi_{j}(t)-\tilde{r}_{j}\right) \rightarrow 0$ when $t \rightarrow \infty$, with $\chi_{j}(t)=\left[\chi_{1 j}(t), \ldots, \chi_{N j}(t)\right]^{\prime}$. Now the control input $u(t)$ still takes the form of 4.29 ) and the dynamics of $\delta(t)$ can be decided as 4.30 , with an extra term $+\left[J_{1, n} \otimes \mathbf{J}_{N}\right]\left[\begin{array}{c}\tilde{r}_{1} \\ \vdots \\ \tilde{r}_{n}\end{array}\right]$ on the righthand side.

Therefore, if the extra term vanishes, which is equivalent to $J_{1, n}\left(\tilde{r}_{i}-\tilde{r}_{j}\right)=\left(\tilde{r}_{i}-\tilde{r}_{j}\right)$ for any $i, j$ and hence $A\left(r_{i}-r_{j}\right)=\left(r_{i}-r_{j}\right)$ for any $i, j$, we can proceed along a similar line of Theorem 4.3 to obtain the following result:

Theorem 4.6. Assume that $A\left(r_{i}-r_{j}\right)=\left(r_{i}-r_{j}\right)$ for any $i, j$. Let the conditions of Theorem 4.3 hold, and moreover $p_{0} \geq \max _{i}\left\|P^{-1}\left(r^{i}-\bar{r}\right)\right\|, \bar{r}=\frac{1}{N} \sum_{i=1}^{N} r^{i}$. Then (4.49) can be achieved with convergence rate $O\left(\gamma^{t}\right)$ in the light of 4.28 and $n$ bits are enough to achieve the formation control.

Similarly, if we define the formation with a leader by $\lim _{t \rightarrow \infty}\left[\left(x^{i}(t)-r^{i}\right)-x^{0}(t)\right]=0, i=1, \ldots, N$, and replace the control input 4.50 for $t \geq n$ with

$$
u_{i}(t)=\sum_{j=1}^{n} k_{j}\left\{\sum_{l \in \mathcal{N}_{i}} w_{i l}\left[\left(\hat{\chi}_{l i j}(t)-\tilde{r}_{l j}\right)-\left(\hat{\chi}_{i j}(t)-\tilde{r}_{i j}\right)\right]+b_{i}\left[\hat{\chi}_{0 i j}(t)-\left(\hat{\chi}_{i j}(t)-\tilde{r}_{i j}\right)\right]\right\}
$$

then we obtain

Theorem 4.7. Assume that $A r_{i}=r_{i}$ for any $i$. Let the conditions of Theorem 4.4 hold and moreover $p_{0} \geq \max _{i}\left\|P^{-1} r^{i}\right\|_{\infty}$. Then the formation with a leader can be achieved with convergence rate $O\left(\gamma^{t}\right)$ in the light of 4.28 and $n$ bits are enough to achieve the formation control. 


\subsection{Numerical Examples}

Consider the sampled position-velocity-acceleration system taking the form of 4.42 with $x^{i}=$ $\left[l_{i}, v_{i}, a_{i}\right]^{\prime}$ and $A=\left[\begin{array}{ccc}1 & T & T^{2} / 2 \\ & 1 & T \\ & 1\end{array}\right], b=\left[T^{3} / 6, T^{2} / 2, T\right]^{\prime}$, where the sampling period $T=0.5 s$. For a group of 4 follower vehicles with a leader moving in $\mathbb{R}^{2}$, we assume that the movement along each direction is independently governed by the above systems, and we desire them to move as a pentagon with the relative position of the followers to the leader specified by $[-5,-5]^{\prime},[-10,-2.5]^{\prime},[-10,2.5]^{\prime},[-5,5]^{\prime}$. It is clear that to maintain such a shape the relative velocity and acceleration to the leader have to converge to 0 . As a result, the formation components of each coordinate for follower agents are re-

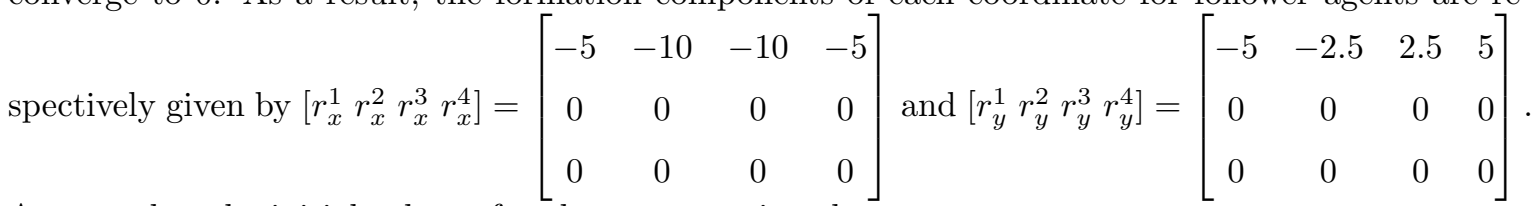
Assume that the initial values of each agent are given by

$$
\begin{aligned}
{\left[x_{1}^{0}(0) x_{1}^{1}(0) x_{1}^{2}(0) x_{1}^{3}(0) x_{1}^{4}(0)\right] } & =\left[\begin{array}{ccccc}
0 & 0 & 1 & 0 & -1 \\
0 & 1 & -1 & 1.5 & -1 \\
0.005 & 0 & -0.1 & 0.21 & -0.1
\end{array}\right], \\
{\left[x_{2}^{0}(0) x_{2}^{1}(0) x_{2}^{2}(0) x_{2}^{3}(0) x_{2}^{4}(0)\right] } & =\left[\begin{array}{ccccc}
0 & 1 & 0 & -1 & 0 \\
0 & 0 & 0.8 & -0.5 & 0 \\
0.005 & 0.1 & -0.1 & 0.11 & -0.1
\end{array}\right] .
\end{aligned}
$$

The vehicles are connected as a circle and the leader 0 communicates to the follower 1 and 4 , with weight 1 on each edge, giving rise to $\bar{\lambda}_{1}=0.3820$ and $c_{1}=1, c_{2}=1, c_{3}=10.8541$. The required transformation matrix in transforming the original system into a triple-integrator system is given by $P=\left[\begin{array}{ccc}T^{3} & T^{3} & T^{3} / 6 \\ & T^{2} & T^{2} / 2 \\ & & T\end{array}\right]$. Moreover, select $\varepsilon=0.01$ and $p_{0}=10$ to satisfy the conditions in Theorem 4.7.

From Fig. 4.1 we can see that the formation can be achieved by 3 bits of information exchange; besides, from Fig. 4.2 and 4.3 we can infer that the velocity and acceleration converge to $0.005 t$ and 0.005 as those of the leader.

\subsection{Conclusion}

In this chapter, we considered the leaderless and leader-following quantized consensus for $n$-th order integrator MASs, where only the first state variable can be measured. We first proposed an observer- 


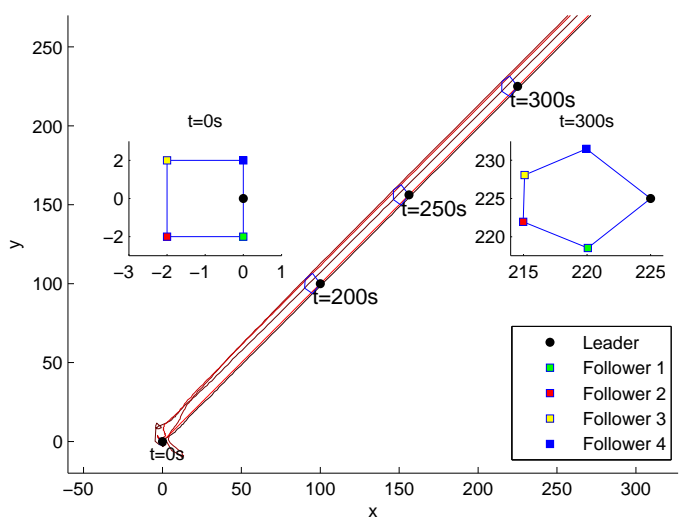

Figure 4.1: Formation of 5 agents on $\mathbb{R}^{2}$.
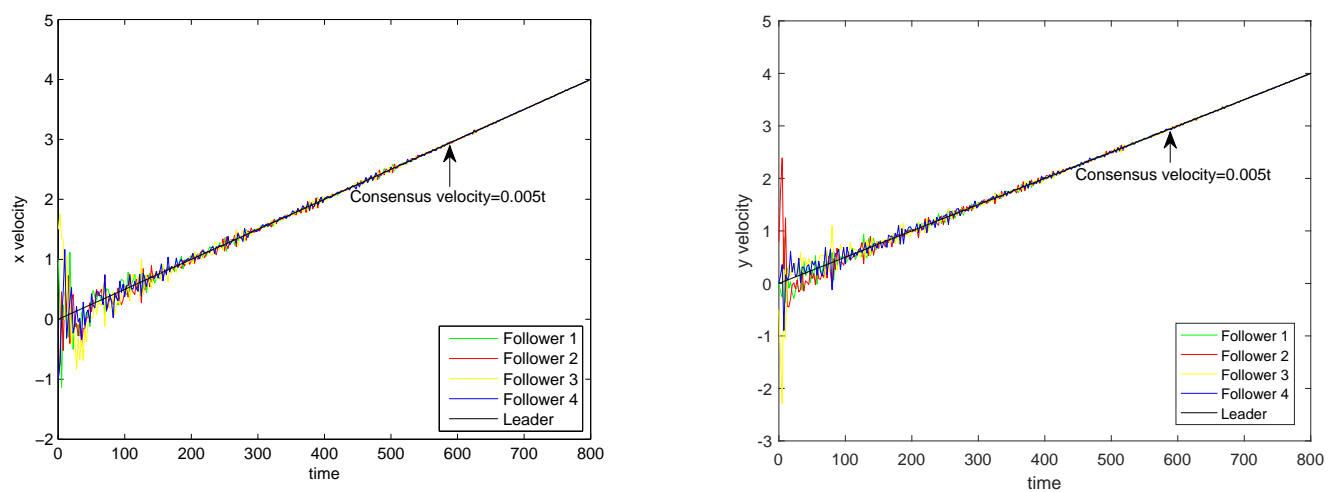

Figure 4.2: Velocity consensus.
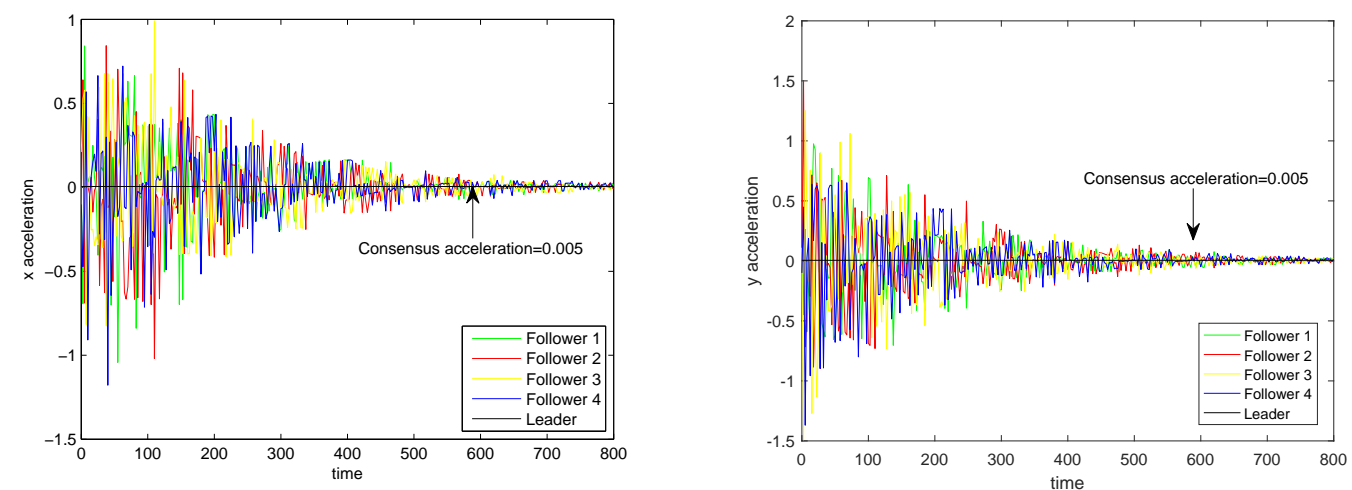

Figure 4.3: Acceleration consensus. 
based encoding-decoding scheme to obtain a delayed estimate of unmeasurable state, and devised a consensus protocol based on the encoder/decoder outputs. By perturbation analysis of matrices, we showed that $n$ bits of information exchange between agents suffice for the leaderless and leaderfollowing quantized consensus with an exponential convergence rate in a fixed and connected network. Then we considered a more general case when each agent is controllable and observable with single input and single output, and their system matrix admits $n$ identical unit eigenvalues. We showed that a same result can be obtained for such systems by applying the encoder/decoder and corresponding consensus protocol to a Luenberger observer. We also discussed the consensus value and an application to formation control, and provided a numerical example for illustration.

\subsection{Proof of Main Lemmas}

Proof of Lemma 4.1. The characteristic polynomial of $A_{i}$ can be written as

$$
\begin{aligned}
p_{i}(\chi) & =\operatorname{det}\left[\chi^{n} I_{n}-\chi^{n-1}\left(J_{1, n}-\lambda_{i} K_{1}\right)+\chi^{n-2} \lambda_{i} K_{2}+\cdots+\chi \lambda_{i} K_{n-1}+\lambda_{i} K_{n}\right] \\
& =\chi^{(n-1)^{2}}\left[\chi^{n-1}(\chi-1)^{n}+\lambda_{i} k_{n}(\chi-1)^{n-1}+\lambda_{i} k_{n-1} \chi(\chi-1)^{n-2}+\cdots+\lambda_{i} k_{1} \chi^{n-1}\right] .
\end{aligned}
$$

Obviously, $A_{i}$ has $(n-1)^{2} 0$ eigenvalues. In what follows, we focus on

$$
f_{i}(\chi)=\chi^{n-1}(\chi-1)^{n}+\lambda_{i} k_{n}(\chi-1)^{n-1}+\lambda_{i} k_{n-1} \chi(\chi-1)^{n-2}+\cdots+\lambda_{i} k_{1} \chi^{n-1},
$$

by which $f_{i}(\chi)$ admits 1 as its root of multiplicity $n$, and 0 as its root of multiplicity $n-1$ when $\varepsilon=0$. In the sequel the cases of eigenvalues 1 and 0 are discussed separately.

(i) The case of eigenvalue 1 . Denote the $n$ perturbed roots of 1 as $\chi^{(1)}=1+\Delta \chi^{(1)}$. Since

$$
\begin{aligned}
\chi^{m-1}(\chi-1)^{n-m} & =[1+(\chi-1)]^{m-1}(\chi-1)^{n-m} \\
& =\sum_{l=0}^{m-1} C(m-1, l)(\chi-1)^{n-m+l}, m=1, \ldots, n, \\
\chi^{n-1}(\chi-1)^{n} & =[1+(\chi-1)]^{n-1}(\chi-1)^{n} \\
& =\sum_{m=0}^{n-1} C(n-1, m)(\chi-1)^{n+l},
\end{aligned}
$$

we have

$$
a_{i m}(\varepsilon)= \begin{cases}\left(c_{n}+c_{n-1}\right) \lambda_{i} \varepsilon+o(\varepsilon), & m=1 \\ c_{n-m+1} \lambda_{i} \varepsilon^{m-1}+o\left(\varepsilon^{m-1}\right), & m=2, \ldots, n\end{cases}
$$




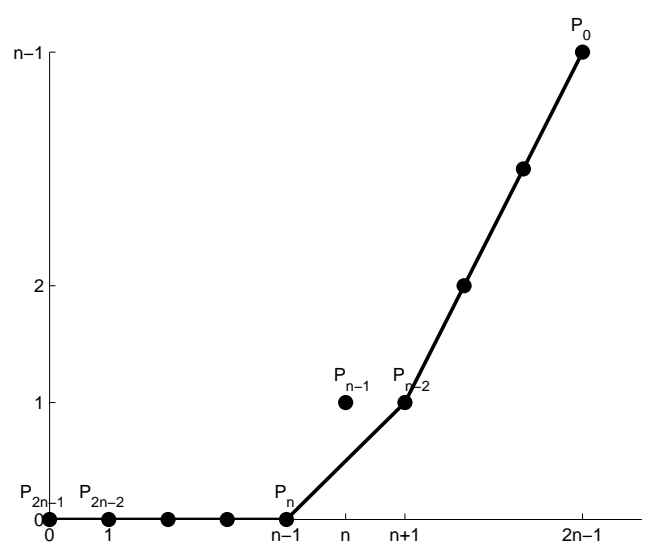

Figure 4.4: Newton diagram.

where $a_{i m}(\varepsilon)$ is the coefficient of the term $(\chi-1)^{n-m}$. Substituting $\chi$ with $1+\Delta \chi^{(1)}$, we obtain

$$
f_{i}\left(1+\Delta \chi^{(1)}\right)=\sum_{m=1}^{n} a_{i m}(\varepsilon)\left(\Delta \chi^{(1)}\right)^{n-m}+\sum_{m=0}^{n-1} C(n-1, m)\left(\Delta \chi^{(1)}\right)^{n+m} .
$$

Now the Newton diagram 108 can be depicted as in Fig. 4.4 which consists of points $P_{2 n-1-j}\left(j, \alpha_{2 n-1-j}\right), j=$ $0, \ldots, 2 n-1$, and the segments on the lower boundary of the convex hull of the plotted points, where $\alpha_{2 n-1-j}$ is the leading exponent of $\varepsilon$ in the coefficient of $\left(\Delta \chi^{(1)}\right)^{2 n-1-j}$. Clearly the slopes of the two non-horizontal segments are $1 / 2,1$ respectively, suggesting that $\Delta \chi^{(1)}$ has two forms of the expansions:

$$
\begin{aligned}
& \Delta \chi^{(1)}=\mu_{1} \varepsilon^{\frac{1}{2}}+o\left(\varepsilon^{\frac{1}{2}}\right), \mu_{1} \neq 0 \\
& \Delta \chi^{(1)}=v_{1} \varepsilon+o(\varepsilon), \quad v_{1} \neq 0 .
\end{aligned}
$$

Substituting them into 4.52 and equating the coefficients of the lowest order terms of $\varepsilon$ lead to that

$$
\left\{\begin{array}{l}
\mu_{1}^{2}+c_{n-1} \lambda_{i}=0 \\
c_{n-1} \lambda_{i} v_{1}^{n-2}+\cdots+c_{2} \lambda_{i} v_{1}+c_{1} \lambda_{i}=0
\end{array}\right.
$$

In order that the $n$ perturbed roots lie inside the unit circle, $\mu_{1}$ must be a pure imaginary number (yielding $c_{n-1}>0$ ) and $v_{1}$ must be negative. Furthermore, the second equation of 4.54) must admit $n-2$ distinct negative real roots, if we require the $n$ perturbed roots to be distinct from each other. To decide the second term of the first expression in 4.53 , we substitute $\Delta \chi^{(1)}=\mu_{1} \varepsilon^{\frac{1}{2}}+\mu_{2} \varepsilon^{\beta}+\cdots$ 
into 4.52 and find the lowest order term

$$
\begin{aligned}
& (n-1) \mu_{1}^{n+1} \varepsilon^{\frac{n+1}{2}}+n \mu_{1}^{n-1} \mu_{2} \varepsilon^{\frac{n-1}{2}+\beta} \\
+ & \left(c_{n}+c_{n-1}\right) \lambda_{i} \mu_{1}^{n-1} \varepsilon^{\frac{n+1}{2}} \\
+ & (n-2) c_{n-1} \lambda_{i} \mu_{1}^{n-3} \mu_{2} \varepsilon^{\frac{n-1}{2}+\beta} \\
+ & c_{n-2} \lambda_{i} \mu_{1}^{n-3} \varepsilon^{\frac{n+1}{2}}=0,
\end{aligned}
$$

which implies $\beta=1$ and

$$
\begin{aligned}
\mu_{2} & =-\frac{c_{n-2} \lambda_{i}+\left(c_{n}+c_{n-1}\right) \lambda_{i} \mu_{1}^{2}+(n-1) \mu_{1}^{4}}{(n-2) c_{n-2} \lambda_{i}+n \mu_{1}^{2}} \\
& =-\frac{1}{2}\left[\left(c_{n}-(n-2) c_{n-1}\right) \lambda_{i}-\frac{c_{n-2}}{c_{n-1}}\right] .
\end{aligned}
$$

Because

$$
\begin{aligned}
\left|\chi^{(1)}\right|^{2} & =1+\left(\left|\mu_{1}\right|^{2}+2 \mu_{2}\right) \varepsilon+o(\varepsilon) \\
& =1-\left[\left(c_{n}-(n-1) c_{n-1}\right) \lambda_{i}-\frac{c_{n-2}}{c_{n-1}}\right] \varepsilon+o(\varepsilon),
\end{aligned}
$$

it suffices to take $\frac{c_{n-2}}{c_{n-1}}<\left(c_{n}-(n-1) c_{n-1}\right) \lambda_{i}$ such that $\left|\chi^{(1)}\right|<1$. We are left to show that $\Omega\left(\lambda_{i}, n\right)$ is not empty. In fact, noticing that the first two inequalities in 4.20 can always be satisfied with sufficiently large $c_{n}$, while 4.19 is independent of $c_{n}$ and its roots can be assigned arbitrarily by selecting proper $c_{n-1}, \ldots, c_{1}$, we obtain the non-emptiness of $\Omega\left(\lambda_{i}, n\right)$.

(ii) The case of eigenvalue 0 . Denote the $n-1$ perturbed roots of 0 as $\chi^{(0)}=\Delta \chi^{(0)}$. Because

$$
\begin{aligned}
\chi^{j-1}(\chi-1)^{n-j} & =\sum_{l=0}^{n-j} C(n-j, l)(-1)^{l} \chi^{n-l-1}, j=1, \ldots, n, \\
\chi^{n-1}(\chi-1)^{n} & =\sum_{l=0}^{n} C(n, l)(-1)^{l} \chi^{2 n-l-1},
\end{aligned}
$$

similar to the case (i), we obtain

$$
\Delta \chi^{(0)}=\vartheta_{1} \varepsilon^{\frac{1}{n-1}}+\cdots
$$

where $\vartheta_{1} \neq 0$ satisfies

$$
(-1)^{n} \vartheta_{1}^{n-1}+(-1)^{n-1} c_{n} \lambda_{i}=0 .
$$

As a result, the solutions are given by

$$
\vartheta_{i l 1}=\left(c_{n} \lambda_{i}\right)^{\frac{1}{n-1}} e^{\frac{2 l \pi}{n-1} \jmath}, l=1, \ldots, n-1,
$$

implying that the $n-1$ perturbed roots of 0 are distinct from each other when $\varepsilon$ is sufficiently small. 
In summary, we obtain the result.

Proof of Lemma 4.2. We are to solve the following Jordan decomposition for $A_{i}$ :

$$
A_{i}=A_{0}+\sum_{j=1}^{n-1} A_{i j} \varepsilon^{j}=R_{i} \tilde{A}_{i} R_{i}^{-1}
$$

To this end, we first decide the Jordan basis of the original matrix $A_{0}$, and then the perturbed Jordan basis, and finally the first $n$ rows of $R_{i}^{-1}$ by adjoint method as required by the Lemma 4.2 .

(i) The Jordan basis of $A_{0}$. The Jordan chain corresponding to the eigenvalue 1 can be determined as

$$
u_{n-1} \stackrel{A_{0}-I_{n}}{\longrightarrow} u_{n-2} \stackrel{A_{0}-I_{n}}{\longrightarrow} \ldots \stackrel{A_{0}-I_{n}}{\longrightarrow} u_{1} \stackrel{A_{0}-I_{n}}{\longrightarrow} u_{0}
$$

where $\left[u_{0} u_{1} \ldots u_{n-1}\right]^{\prime}=\left[U_{1} U_{2} \ldots U_{n}\right] \in \mathbb{R}^{n \times n^{2}}$ can be divided into $n$ lower triangular submatrices $U_{j} \in \mathbb{R}^{n \times n}:$

$$
\begin{aligned}
& U_{1}=I_{n} \\
& {\left[U_{j}\right]_{s, t}=\left\{\begin{array}{l}
(-1)^{s-t} C(j-2+s-t, j-2), \\
s-t=0,1, \ldots, n-1, j=2, \ldots, n ; \\
0, s-t<0 .
\end{array}\right.}
\end{aligned}
$$

We denote the eigenspace with respect to eigenvalue 1 by

$$
S_{1}=\operatorname{span}\left\{u_{0}, u_{1}, \ldots, u_{n-1}\right\}
$$

As to the eigenvalue 0 , there exist $n$ Jordan chains of length $n-1$ :

$$
\begin{array}{ccccc}
e_{n+1} & \stackrel{A_{0}}{\longrightarrow} \ldots \stackrel{A_{0}}{\longrightarrow} & e_{(n-2) n+1} & \stackrel{A_{0}}{\longrightarrow} & e_{(n-1) n+1}, \\
\ldots & \stackrel{A_{0}}{\longrightarrow} \ldots \stackrel{A_{0}}{\longrightarrow} & \ldots & \stackrel{A_{0}}{\longrightarrow} & \ldots \\
e_{n+n-1} & \stackrel{A_{0}}{\longrightarrow} \ldots \stackrel{A_{0}}{\longrightarrow} & e_{(n-2) n+n-1} & \stackrel{A_{0}}{\longrightarrow} & e_{(n-1) n+n-1}, \\
e_{n+n} & \stackrel{A_{0}}{\longrightarrow} \ldots \stackrel{A_{0}}{\longrightarrow} & e_{(n-2) n+n} & \stackrel{A_{0}}{\longrightarrow} & e_{(n-1) n+n},
\end{array}
$$

or equally, $e_{(n-2-j) n+k} \stackrel{A_{0}}{\longrightarrow} e_{(n-1-j) n+k}, j=0, \ldots, n-3, k=1, \ldots, n$. Here $e_{l}$ denotes the vector with a 1 in the $l$-th coordinate and 0 's elsewhere. For convenience we denote

$$
Y_{j}=\operatorname{span}\left\{e_{(n-1-j) n+1} \ldots, e_{(n-1-j) n+n}\right\}, j=0, \ldots, n-2,
$$

as the linear span of the generalized eigenvectors associated with eigenvalue 0 , of $j$-th order; and

$$
S_{0}=Y_{0} \oplus \cdots \oplus Y_{n-2}
$$


as the linear span of all the generalized eigenvectors associated with eigenvalue 0 .

(ii) The perturbed Jordan basis. Below we shall find the perturbed Jordan basis based on the unperturbed basis as obtained in (i). The eigenvector $w_{i l}^{(1)}$ corresponding to the perturbed root $\chi_{i l}^{(1)}$ around 1 is given by the following form of Puiseux series [109]:

$$
\begin{aligned}
& \chi_{i 1}^{(1)}=1+\sum_{m=1}^{\infty} \mu_{i l m} \varepsilon^{m / 2}, \\
& w_{i l}^{(1)}=w_{i l 0}^{(1)}+\sum_{m=1}^{\infty} w_{i l m}^{(1)} \varepsilon^{m / 2}, l=1,2 ; \\
& \chi_{i l}^{(1)}=1+\sum_{m=1}^{\infty} v_{i m l} \varepsilon^{m}, \\
& w_{i l}^{(1)}=w_{i l 0}^{(1)}+\sum_{m=1}^{\infty} w_{i l m}^{(1)} \varepsilon^{m}, l=3, \ldots, n,
\end{aligned}
$$

where $\mu_{i l 1}$ and $v_{i 2 l}=v_{2 l}$ have been defined in Lemma 4.1. To find the eigenvector $w_{i l}^{(1)}$, we substitute $\chi_{i l}^{(1)}, w_{i l}^{(1)}$ into the equation $A_{i 1} w_{i l}^{(1)}=\chi_{i l}^{(1)} w_{i l}^{(1)}$, and collect coefficients of the term with equal power of $\varepsilon$. Moreover, noticing the fact that $A_{i j} u_{m}=0$ for $j=1, \ldots, n-2 ; m=0, \ldots, n-2-j$, where $A_{i j}$ has been defined in 4.59 and imposing the normalization condition as

$$
v_{n-1}^{\prime} w_{i l}^{(1)}=1
$$

where $v_{n-1}^{\prime}$ is the left associated eigenvector of $A_{0}$ with respect to eigenvalue 1 of $(n-1)$-th order, satisfying $v_{n-1}^{\prime} u_{0}=1, v_{n-1}^{\prime} u_{1}=\cdots=v_{n-1}^{\prime} u_{n-1}=0$, we get

$$
\begin{array}{ll}
w_{i l}^{(1)}=u_{0}+\sum_{m=1}^{n-1} \varepsilon^{m / 2}\left(\mu_{i l 1}^{m} u_{m}+\bar{u}_{i l m}\right)+\varepsilon^{n / 2}\left(\mu_{i l 1}^{n} y_{n}+\bar{u}_{i l n}\right)+O\left(\varepsilon^{(n+1) / 2}\right), & l=1,2 ; \\
w_{i l}^{(1)}=u_{0}+\sum_{m=1}^{n-1} \varepsilon^{m}\left(v_{l 1}^{m} u_{m}+\bar{u}_{i l m}\right)+\varepsilon^{n}\left(v_{i l 1}^{n} y_{n}+\bar{u}_{i l n}\right)+O\left(\varepsilon^{n+1}\right), & l=3, \ldots, n,
\end{array}
$$

where $\bar{u}_{i l 1}=0, \bar{u}_{i l m} \in \operatorname{span}\left\{u_{1}, \ldots, u_{m-1}\right\}, m=2, \ldots, n, l=1, \ldots, n$ and $y_{n} \in S_{0}$.

The eigenvectors corresponding to the $n-1$ different perturbed eigenvalues around 0 can be obtained in a similar way. Now we have

$$
\begin{aligned}
& \chi_{i l}^{(0)}=\sum_{m=1}^{\infty} \vartheta_{i l m} \varepsilon^{m /(n-1)}, \\
& w_{i l}^{(0)}=w_{i l 0}^{(0)}+\sum_{m=1}^{\infty} w_{i l m}^{(0)} \varepsilon^{m /(n-1)}, l=1, \ldots, n-1,
\end{aligned}
$$

where $\vartheta_{i l 1}$ has been defined in 4.58). According to $110, w_{i l 0}^{(0)}$ satisfies $C_{i} w_{i l 0}^{(0)}=c_{n} \lambda_{i} w_{i l 0}^{(0)}$, where $C_{i}$ is a linear operator on $Y_{0}$. Written in a matrix form, the $(s, t)$ entry of $C_{i}$ is defined by $C_{i}(s, t)=$ $\left\langle A_{i 1} e_{(n-1) n+t}, f_{s}\right\rangle, s, t=1,2, \ldots, n$, where $\left\{f_{1}, \ldots, f_{n}\right\}$ is a biorthogonal basis in the left eigenspace 
of $A_{0}$ corresponding to eigenvalue 0 such that $\left\langle e_{n+t}, f_{s}\right\rangle=\delta_{s t}$. Direct computation shows that

$$
C_{i}(s, t)= \begin{cases}c_{n} \lambda_{i}(-1)^{n-s}, & s=1, \ldots, n, t=n \\ 0, & \text { otherwise }\end{cases}
$$

and $w_{i l 0}^{(0)}$ can be chosen as $w_{i l 0}^{(0)}=w_{0}=\sum_{k=1}^{n}(-1)^{k-1} e_{(n-1) n+k}$ for any $l$. Imposing the normalization condition as $P_{0}(w)=w_{0}$, with $P_{0}$ being the projection on $Y_{0}$ along $Y_{1} \oplus \cdots \oplus Y_{n-2} \oplus S_{1}$, we get

$$
\begin{aligned}
w_{i l m}^{(0)} & =\vartheta_{i l 1}^{m} \sum_{k=1}^{n}(-1)^{k-1} e_{(n-m-1) n+k}+\bar{y}_{i l m}, m=1, \ldots, n-2 ; \\
w_{i l, n-1}^{(0)} & =\vartheta_{i l 1}^{n-1} \sum_{k=1}^{n}(-1)^{k-1} u_{n-k}+\bar{y}_{i l(n-1)},
\end{aligned}
$$

where $\bar{y}_{i l 1}=0$ and $\bar{y}_{i l m} \in Y_{1} \oplus \cdots \oplus Y_{m-1}, m=2, \ldots, n-1$.

As to the other zero eigenvalues, the original Jordan structure is partially preserved as follows 111:

$$
\begin{aligned}
& \tilde{e}_{n+1} \quad \stackrel{A_{i}}{\longrightarrow} \ldots \stackrel{A_{i}}{\longrightarrow} \quad \tilde{e}_{(n-2) n+1} \quad \stackrel{A_{i}}{\longrightarrow} \quad \tilde{e}_{(n-1) n+1}, \\
& \ldots \quad \stackrel{A_{i}}{\longrightarrow} \ldots \stackrel{A_{i}}{\longrightarrow} \quad \ldots \quad \stackrel{A_{i}}{\longrightarrow} \quad \ldots \\
& \tilde{e}_{n+n-2} \stackrel{A_{i}}{\longrightarrow} \ldots \stackrel{A_{i}}{\longrightarrow} \quad \tilde{e}_{(n-2) n+n-2} \stackrel{A_{i}}{\longrightarrow} \quad \tilde{e}_{(n-1) n+n-2}, \\
& \tilde{e}_{n+n-1} \stackrel{A_{i}}{\longrightarrow} \ldots \stackrel{A_{i}}{\longrightarrow} \tilde{e}_{(n-2) n+n-1} \stackrel{A_{i}}{\longrightarrow} \tilde{e}_{(n-1) n+n-1}, \\
& \tilde{e}_{j n+k}=\left\{\begin{array}{l}
e_{j n+k}, k \leq j \\
e_{j n+k}-\frac{c_{k}}{c_{n}} \varepsilon^{n-k-1} e_{(n-(k-j)) n+n}, \\
k-j=1, \ldots, n-2
\end{array}\right. \\
& 1 \leq j, k \leq n-1
\end{aligned}
$$

(iii) The Jordan decomposition of $A_{i}$. Assume that the Jordan normal form of $A_{i}$ is given by

$$
\tilde{A}_{i}=\operatorname{diag}\left\{\chi_{i 1}^{(1)}, \ldots, \chi_{i n}^{(1)}, \chi_{i 1}^{(0)}, \ldots, \chi_{i(n-1)}^{(0)}, J_{0, n-1}, \ldots, J_{0, n-1}\right\}
$$

where the total number of $J_{0, n-1}$ is $n-1$. Accordingly $R_{i}$ is given by the block matrix

$$
R_{i}=\left[\begin{array}{lllll}
W_{i}^{(1)} & W_{i}^{(0)} & \tilde{P}_{1} & \ldots & \tilde{P}_{n-1}
\end{array}\right]
$$

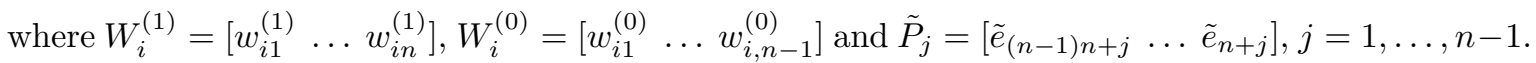
By 4.65 it is clear that each column of $\tilde{P}_{j}$ is a linear combination of $e_{l}$ 's, $l \geq n+1$, and consequently each row in the first $n$ rows of $R_{i}$, is an $n^{2}$-dimensional row vector with the first $2 n-1$ columns as its only possible nonzero entries. Therefore, it is enough to only focus on the first $2 n-1$ rows of $R_{i}^{-1}$. We will use the example of $n=3$ to illustrate how to estimate the magnitude of each entry in the 
first $2 n-1$ rows of $R_{i}^{-1}$. The columns of $R_{i}$ are given in a left-to-right sequence as follows:

$$
\begin{aligned}
w_{i 1}^{(1)}= & e_{1}+\varepsilon^{1 / 2} \mu_{i 11} e_{2}+\varepsilon \mu_{i 11}^{2} e_{3} \\
& +e_{4}+e_{7}+O\left(\varepsilon^{1 / 2}\right)\left(e_{5}+e_{8}\right)+O(\varepsilon)\left(e_{6}+e_{9}\right), \\
w_{i 2}^{(1)}= & e_{1}+\varepsilon^{1 / 2} \mu_{i 21} e_{2}+\varepsilon \mu_{i 21}^{2} e_{3} \\
& +e_{4}+e_{7}+O\left(\varepsilon^{1 / 2}\right)\left(e_{5}+e_{8}\right)+O(\varepsilon)\left(e_{6}+e_{9}\right), \\
w_{i 3}^{(1)}= & e_{1}+\varepsilon v_{31} e_{2}+\varepsilon^{2} v_{31}^{2} e_{3} \\
& +e_{4}+e_{7}+O(\varepsilon)\left(e_{5}+e_{8}\right)+O\left(\varepsilon^{2}\right)\left(e_{6}+e_{9}\right) ; \\
w_{i 1}^{(0)}= & \left(e_{7}-e_{8}+e_{9}\right)+\varepsilon^{1 / 2} \vartheta_{i 11}\left(e_{4}-e_{5}+e_{6}\right)+O(\varepsilon), \\
w_{i 2}^{(0)}= & \left(e_{7}-e_{8}+e_{9}\right)+\varepsilon^{1 / 2} \vartheta_{i 21}\left(e_{4}-e_{5}+e_{6}\right)+O(\varepsilon) ; \\
\tilde{e}_{7}= & e_{7}, \quad \tilde{e}_{4}=e_{4} ; \\
\tilde{e}_{8}= & e_{8}, \quad \tilde{e}_{5}=e_{5}-\frac{c_{2}}{c_{4}} \varepsilon e_{9} .
\end{aligned}
$$

Clearly, $\operatorname{det} R_{i}$ is a Puiseux series of $\varepsilon$ and its order can be obtained by taking out terms with $\varepsilon^{1 / 2} e_{2}$ and $\varepsilon e_{3}$ respectively from $w_{i 1}^{(1)}$ and $w_{i 2}^{(1)}$, terms with $e_{1}$ from $w_{i 3}^{(1)}$, terms with $e_{6}$ and $e_{9}$ from $w_{i 1}^{(0)}$ and $w_{i 2}^{(0)}$, and terms with $e_{7}, e_{4}, e_{8}, e_{5}$ respectively from $\tilde{e}_{7}, \tilde{e}_{4}, \tilde{e}_{8}, \tilde{e}_{5}$. As a result,

$$
\left|\operatorname{det} R_{i}\right|=\left|\mu_{i 11} \mu_{i 21}\left(\mu_{i 21}-\mu_{i 11}\right)\right|\left|\operatorname{det} V\left(\vartheta_{i 11}, \vartheta_{i 21}\right)\right| \varepsilon^{2}(1+o(1)) \text {, }
$$

where $V(u, v)=\left[\begin{array}{ll}1 & u \\ 1 & v\end{array}\right]$ is a Vandermonde matrix of order 2. For the first row in $R_{i}^{-1}$ (which is obtained by deleting the first column $\left.w_{i 1}^{(1)}\right)$, if we obtain the cofactor by deleting any one of the unaltered basis in the original rows of $A_{0}$ (namely $\left.e_{7}^{\prime}, e_{4}^{\prime}, e_{8}^{\prime}\right)$, then one of the rest columns will vanish and the cofactor is 0 ; if we obtain the cofactor by deleting one of $e_{5}^{\prime}, e_{6}^{\prime}, e_{9}^{\prime}$, then the altered $w_{i 1}^{(0)}$ and $w_{i 2}^{(0)}$ will contribute at least $\varepsilon^{3 / 2}$ to the cofactor, while the altered $w_{i 1}^{(2)}$ and $w_{i 2}^{(3)}$ will contribute at least $\varepsilon^{1 / 2}$, leading to a cofactor whose order is at least the same as $\operatorname{det} R_{i}$; finally, the cofactor that has the uniquely least order is obtained by deleting $e_{3}^{\prime}$, and resultantly

$$
\left|R_{i}^{-1}(1,3)\right|=\varepsilon^{-1}(1+o(1)) /\left|\mu_{i 11}\left(\mu_{i 21}-\mu_{i 11}\right)\right|
$$

Similarly, the 3-rd entries in the second and third row of $R_{i}^{-1}$ have the largest leading exponent of $\varepsilon^{-1}$ and

$$
\begin{aligned}
& \left|R_{i}^{-1}(2,3)\right|=\varepsilon^{-1}(1+o(1)) /\left|\mu_{i 21}\left(\mu_{i 21}-\mu_{i 11}\right)\right|, \\
& \left|R_{i}^{-1}(3,3)\right|=\varepsilon^{-1}(1+o(1)) /\left|\mu_{i 11} \mu_{i 21}\right| .
\end{aligned}
$$


As for the 4-th and 5-th rows, proceeding as in the above we can conclude that the leading term of each entry in these 2 rows will not exceed $\varepsilon^{-1 / 2}$. In general, the following estimations are valid:

For $l=1,2$,

$$
\left|R_{i}^{-1}(l, j)\right|=\left\{\begin{array}{l}
\frac{1}{2\left|\mu_{i 11}\right|^{n-1}} \varepsilon^{-(n-1) / 2}(1+o(1)), j=n \\
o\left(\varepsilon^{-(n-1) / 2}\right), j \neq n
\end{array}\right.
$$

For $l=3, \ldots, n$,

$$
\left|R_{i}^{-1}(l, j)\right|= \begin{cases}\frac{\varepsilon^{-(n-2)}}{\left|\mu_{i 11}\right|^{2} \prod_{s \geq 3, s \neq l}\left|v_{s 1}-v_{l 1}\right|}(1+o(1)), & j=n \\ o\left(\varepsilon^{-(n-2)}\right), & j \neq n\end{cases}
$$

For $n+1 \leq l \leq 2 n-1,1 \leq j \leq n^{2},\left|R_{i}^{-1}(l, j)\right| \leq O\left(\varepsilon^{-\frac{n-2}{n-1}}\right)$.

Moreover, the first $n$ rows of $R_{i}$ are as follows:

$$
R_{i}(l, j)=\left\{\begin{array}{l}
\mu_{i 1 l}^{j-1} \varepsilon^{(j-1) / 2}+O\left(\varepsilon^{j / 2}\right), 1 \leq l \leq 2 \\
v_{1 l}^{j-1} \varepsilon^{j-1}+O\left(\varepsilon^{j}\right), 3 \leq l \leq n \\
(-1)^{n-j} \varepsilon+O\left(\varepsilon^{2}\right), n+1 \leq l \leq 2 n-1 \\
0, l \geq 2 n
\end{array}\right.
$$

for $j=1, \ldots, n$. Now we can obtain

$$
\begin{aligned}
\left|\xi_{t, j}^{i}\right| \leq & \rho_{i}^{t}\|\xi\|_{\infty}\left[\left[\sum_{l=1}^{n}\left|R_{i}(j, l) R_{i}^{-1}(l, n)\right|(1+o(1))+O\left(\varepsilon^{1-\left(\frac{n-2}{n-1}\right)}\right)\right]\right. \\
\leq & \rho_{i}^{t}\|\xi\|_{\infty}\left[\sum_{l=1}^{2} \frac{\left|\mu_{i 1 l}^{j-1}\right|}{2\left|\mu_{i 11}\right|^{n-1}} \varepsilon^{(j-n) / 2}\right. \\
& \left.+\sum_{l=3}^{n} \frac{\left|v_{1 l}^{j-1}\right|}{\left|\mu_{i 11}\right|^{2} \prod_{s \geq 3, s \neq l}\left|v_{s 1}-v_{l 1}\right|} \varepsilon^{j-(n-1)}\right](1+o(1))
\end{aligned}
$$

and the conclusion follows by comparing $j-(n-1)$ and $\frac{j-n}{2}$, then choosing the term with a smaller exponent. 


\section{Chapter 5}

\section{Quantized Consensus of $2 m$-th Order Oscillator Systems under}

\section{Data Rate Constraint}

As an extension of Chapter 4, in this chapter we focus on the quantized consensus of MASs with another kind of critical high-order dynamics. In specific, we study the consensus problem of $2 m$-th order oscillator systems under data rate constraint, and explore the lowest possible sufficient data rate to achieve the asymptotic consensus. The state matrix of each agent is a $2 m$-th order real Jordan block admitting $m$ identical pairs of conjugate poles on the unit circle; each agent has a single input, and only the first state variable can be measured. Note that here we only focus on the leaderless consensus of the specific oscillator dynamics; however, proceeding along a similar line as in Chapter 4 it is not difficult to extend the results to leader-following consensus and the case of more general dynamics.

Although we employ similar perturbation techniques in the analysis of consensus and data rate as Chapter 4 , the problem posed here is much different, and it is much more challenging to obtain an explicit form of the sufficient data rate. First of all, the encoder/decoder design for integrators no longer applies in the case of oscillators, due to the loss of the special integrator structure. Therefore, we propose a novel observer-based encoding scheme based on the constructability to obtain an instant estimation of the unmesurable state, in contrast to the delayed estimation in the integrator case. However, such an encoding scheme introduces control inputs into the estimation error, which makes the consensus analysis more complicated. Secondly, the perturbation analysis of the characteristic equation and Jordan decomposition is much more challenging for complex eigenvalues. Thirdly, to 
find an explicit form of the required data rate, it needs to calculate a linear combination of some rows of a matrix which is a multiplication of the $(2 m-1)$-th power of the system matrix and the inverse of the observability matrix, and it is unlikely to be obtained by direct computation. To overcome such a challenge, we transform the linear combination into a set of linear equations and resort to combinatorics techniques. We finally manage to show that a data rate between $m$ and $2 m$, depending on the oscillation frequency, suffices to achieve the consensus.

The rest of the chapter is organized as follows. The problem is first formulated in Section 5.1 Under a balanced network which contains a spanning tree, the data rate problem for distributed consensus of the coupled harmonic oscillators $(m=1)$ is investigated in Section 5.2 . The general case of $m \geq 2$ under a fixed and undirected network is studied in Section 5.3 For illustration, two numerical examples are respectively given for the harmonic oscillators and general cases in Section 5.4. Some concluding remarks are drawn in Section 5.5. and the proofs of the main lemmas can be found in Section 5.6 .

\subsection{Problem Formulation}

Consider an MAS in the following form:

$$
\left\{\begin{aligned}
x^{i}(t+1) & =A x^{i}(t)+b u_{i}(t), \\
y_{i}(t) & =x_{i 1}(t)
\end{aligned}\right.
$$

where $x^{i}(t)=\left[x_{i 1}(t), x_{i 2}(t), \ldots, x_{i, 2 m}(t)\right]^{\prime} \in \mathbb{R}^{2 m}, y_{i}(t), u_{i}(t) \in \mathbb{R}$, respectively represent the state, output and input of agent $i, i=1, \ldots, N$. Moreover, $A=\left[\begin{array}{cccc}Q & I_{2} & & \\ & Q & \ddots & \\ & & \ddots & I_{2} \\ & & Q\end{array}\right] \in \mathbb{R}^{2 m \times 2 m}$ is a real Jordan form consisting of $m$ pairs of conjugate eigenvalues $\cos \theta \pm \jmath \sin \theta$ with $\sin \theta \neq 0$ and $Q=\left[\begin{array}{cc}\cos \theta & \sin \theta \\ -\sin \theta & \cos \theta\end{array}\right] ; b=[0, \ldots, 0,1]^{\prime} \in \mathbb{R}^{2 m}$.

As in Chapter 4, we adopt the finite-level uniform quantizer (4.2), and it is enough to use $\left\lceil\log _{2}(2 M(t))\right\rceil$ bits to represent all the signals by remembering Remark 4.1. The problem of distributed quantized consensus is solved if we can design a distributed control protocol with an encoding-decoding scheme, making the states of different agents reach the agreement asymptotically:

$$
\lim _{t \rightarrow \infty}\left[x^{i}(t)-x^{j}(t)\right]=0, \quad i, j=1,2, \ldots, N
$$




\subsection{Harmonic Oscillator Case}

In this section, we will start with the harmonic oscillator case as an example to investigate how many bits of information exchange are enough to achieve consensus exponentially fast with quantized neighbor-based control. We separate it from higher-order cases due to its speciality and simplicity: the solution of this basic case not only provides a result under a directed communication topology, but also serves to facilitate the understanding of higher-order cases. Some relevant remarks will be included in the next section, as a comparison between second-order and higher-order cases, or a summary of both cases. Note that now the system matrix $A=\left[\begin{array}{cc}\cos \theta & \sin \theta \\ -\sin \theta & \cos \theta\end{array}\right]$.

\subsubsection{Encoding-decoding Scheme and Distributed Control Law}

An encoding-decoding scheme is of paramount importance in the quantized consensus, which should not only provide estimates for all the states from the partially measurable states, but also help reduce the data rate. Accordingly, the encoder should serve as an observer, and be based on iterations. To be specific, inspired by the constructability in the sense that the present state of the system can be recovered from the present and past outputs and inputs, namely

$$
\left[\begin{array}{c}
x_{i 1}(t) \\
x_{i 2}(t)
\end{array}\right]=\left[\begin{array}{cc}
0 & 1 \\
-\csc \theta & \cot \theta
\end{array}\right]\left[\begin{array}{c}
x_{i 1}(t-1) \\
x_{i 1}(t)
\end{array}\right]+\left[\begin{array}{c}
0 \\
u_{i}(t-1)
\end{array}\right]
$$

we propose the following encoder $\varphi_{i}$ for agent $i$ :

$$
\left\{\begin{aligned}
s_{i}(1) & =q_{t}\left(\frac{y_{i}(1)}{p(0)}\right), \hat{x}_{i 1}(1)=p(0) s_{i}(1) \\
s_{i}(2) & =q_{t}\left(\frac{y_{i}(2)}{p(1)}\right), \hat{x}_{i 1}(2)=p(1) s_{i}(2) \\
\hat{x}_{i 2}(2) & =\cot \theta \hat{x}_{i 1}(2)-\csc \theta \hat{x}_{i 1}(1) \\
s_{i}(t) & =q_{t}\left(\frac{y_{i}(t)-\left[\cos \theta \hat{x}_{i 1}(t-1)+\sin \theta \hat{x}_{i 2}(t-1)\right]}{p(t-1)}\right) \\
\hat{x}_{i 1}(t) & =\cos \theta \hat{x}_{i 1}(t-1)+\sin \theta \hat{x}_{i 2}(t-1)+p(t-1) s_{i}(t), \\
\hat{x}_{i 2}(t) & =\cot \theta \hat{x}_{i 1}(t)-\csc \theta \hat{x}_{i 1}(t-1), t \geq 2
\end{aligned}\right.
$$

where $p(t)=p_{0} \gamma^{t}, 0<\gamma<1$ is a decaying scaling function.

After $s_{i}(t)$ is received by one of the $i$-th agent's out-neighbors, say $v \in \mathcal{N}_{i}^{-}$, a decoder $\varphi_{i v}$ will 
be activated:

$$
\left\{\begin{array}{l}
\hat{x}_{i v 1}(1)=p(0) s_{i}(1) \\
\hat{x}_{i v 1}(2)=p(1) s_{i}(2), \hat{x}_{i v 2}(2)=\cot \theta \hat{x}_{i v 1}(2)-\csc \theta \hat{x}_{i v 1}(1) \\
\hat{x}_{i v 1}(t)=\cos \theta \hat{x}_{i v 1}(t-1)+\sin \theta \hat{x}_{i v 2}(t-1)+p(t-1) s_{i}(t), \\
\hat{x}_{i v 2}(t)=\cot \theta \hat{x}_{i v 1}(t)-\csc \theta \hat{x}_{i v 1}(t-1), t \geq 2 .
\end{array}\right.
$$

Based on the outputs of the encoding-decoding scheme, the distributed control law of agent $i$ is given by

$$
u_{i}(t)=\left\{\begin{array}{l}
0, t=0,1 \\
\sum_{j=1}^{2} k_{j} \sum_{l \in \mathcal{N}_{i}^{+}} w_{i l}\left[\hat{x}_{l i j}(t)-\hat{x}_{i j}(t)\right], t \geq 2 .
\end{array}\right.
$$

Remark 5.1. As in the integrator case, a scaled "prediction error" is quantized to generate the signal $s_{i}(t)$, in an effort to reduce the number of quantization levels. $s_{i}(t)$ is then used to construct the estimate $\hat{x}_{i 1}(t)$ of the first component $x_{i 1}(t)$, which is combined with $\hat{x}_{i 1}(t-1)$ to obtain the estimate $\hat{x}_{i 2}(t)$ for $x_{i 2}(t)$. Denote $\Delta_{i}(t)=s_{i}(t)-d_{i}(t)$ as the quantization error, where

$$
d_{i}(t)=\left\{\begin{array}{l}
\frac{y_{i}(t)}{p(t-1)}, t=1,2 \\
\frac{y_{i}(t)-\left[\cos \theta \hat{x}_{i 1}(t-1)+\sin \theta \hat{x}_{i 2}(t-1)\right]}{p(t-1)}, t>2,
\end{array}\right.
$$

and $e_{i j}(t)=\hat{x}_{i j}(t)-x_{i j}(t)$ as the estimation error for $x_{i j}(t), j=1,2$. Then comparing (5.3), 5.4) and 5.5) we have

$$
\left\{\begin{array}{l}
e_{i 1}(t)=\hat{x}_{i 1}(t)-x_{i 1}(t)=p(t-1) \Delta_{i}(t), t \geq 1 \\
e_{i 2}(t)=\hat{x}_{i 2}(t)-x_{i 2}(t)=\cot \theta e_{i 1}(t)-\csc \theta e_{i 1}(t-1)-u_{i}(t-1), t \geq 2
\end{array}\right.
$$

Note that now the estimation error is related with control inputs in addition to quantization errors, which is different from 4.13 in the integrator case and may impair the consensus result. But as shown in the consensus analysis below, the influence of the control inputs can be ignored with arbitrarily small control gains. 


\subsubsection{Consensus Analysis and Data Rate}

Some notations are defined as follows:

$$
\begin{aligned}
u(t) & =\left[u_{1}(t), \ldots, u_{N}(t)\right]^{\prime}, \\
\Delta(t) & =\left[\Delta_{1}(t), \ldots, \Delta_{N}(t)\right]^{\prime}, \\
d(t) & =\left[d_{1}(t), \ldots, d_{N}(t)\right]^{\prime}, \\
x_{j}(t) & =\left[x_{1 j}(t), \ldots, x_{N j}(t)\right]^{\prime}, \\
\delta_{j}(t) & =\left(I_{N}-\phi_{1} \psi_{1}^{\prime}\right) x_{j}(t)=\left[\delta_{1 j}(t), \ldots, \delta_{N j}(t)\right]^{\prime}, \\
e_{j}(t) & =\left[e_{1 j}(t), \ldots, e_{N j}(t)\right]^{\prime},
\end{aligned}
$$

where $\phi_{1}=\frac{1}{\sqrt{N}} \mathbf{1}_{N}$ and $\psi_{1}$ is the corresponding left eigenvector of Laplacian $L$, satisfying $\psi_{1}^{\prime} \phi_{1}=1$.

We make the following two assumptions for the subsequent analysis.

Assumption 5.1. The communication graph $\mathcal{G}$ contains a spanning tree.

Assumption 5.2. There exist known positive constants $C^{*}$ and $C_{\delta}^{*}$ such that $\max _{j=1,2}\left\|x_{j}(0)\right\|_{\infty} \leq C^{*}$ and $\max _{j=1,2}\left\|\delta_{j}(0)\right\|_{\infty} \leq C_{\delta}^{*}$.

Remark 5.2. Assumption 5.1 is a standard assumption, under which we can find a nonsingular matrix $T_{L}=\left[\begin{array}{llll}\phi_{1} & \phi_{2} & \ldots & \phi_{N}\end{array}\right]$ with $\phi_{1}=\frac{1}{\sqrt{N}} \mathbf{1}_{N}$, such that $T_{L}^{-1} L T_{L}=\operatorname{diag}\left\{J_{0, N_{1}}, J_{\lambda_{2}, N_{2}}, \ldots, J_{\lambda_{l}, N_{l}}\right\} \triangleq L_{J}$, satisfying $0<R e \lambda_{2} \leq \cdots \leq$ Re $\lambda_{l}$ with 0 as a simple eigenvalue, i.e $N_{1}=1$. Note that we denote $T_{L}^{-1}(i, \cdot)=\psi_{i}^{\prime}, i=1, \ldots, N$. Assumption 5.2 enables us to make the quantizer $q_{t}(\cdot)$ unsaturated at initial steps.

The following lemma is critical in the consensus analysis.

Lemma 5.1. Denote $K=\left[\begin{array}{cc}0 & 0 \\ k_{1} & k_{2}\end{array}\right]$ and $A_{i}=A-\lambda_{i} K$ with $\operatorname{Re} \lambda_{i}>0$. Let $k_{j}=c_{j} \varepsilon, j=1,2$. Then the following results hold with sufficiently small $\varepsilon>0$ :

1). The spectral radius $\rho_{i}$ of $A_{i}$ is less than 1 if $c_{2} \cos \theta-c_{1} \sin \theta>0$ and $c_{1} \cos \theta+c_{2} \sin \theta=0$. Moreover, $\rho_{i}=1-\frac{1}{2}\left(\operatorname{Re} \lambda_{i}\right)\left(c_{2} \cos \theta-c_{1} \sin \theta\right) \varepsilon+o(\varepsilon)$.

2). Take $c_{1}, c_{2}$ as in 1$)$. For any vector $\xi \in \mathbb{R}^{2}$, the entries of $A_{i}^{t} \xi, t \in \mathbb{N}^{+}$, which are denoted as $\xi_{t, 1}^{i}$ and $\xi_{t, 2}^{i}$, satisfy that $\left|\xi_{t, j}^{i}\right| \leq 5 / 2 \rho_{i}^{t}$ for $j=1,2$.

Proof. 1). Noticing that $A=P\left[\begin{array}{ll}e^{\jmath \theta} & \\ & e^{-\jmath \theta}\end{array}\right] P^{-1}$ with $P=\left[\begin{array}{cc}1 & 1 \\ \jmath & -\jmath\end{array}\right]$ and $P^{-1}=\frac{1}{2}\left[\begin{array}{cc}1 & -\jmath \\ 1 & \jmath\end{array}\right]$, we have

$$
\begin{aligned}
\mu I-A & =P\left[\begin{array}{cc}
\mu-e^{\jmath \theta} & \\
\mu-e^{-\jmath \theta}
\end{array}\right] P^{-1} \\
& =\frac{1}{2}\left[\begin{array}{cc}
\left(\mu-e^{\jmath \theta}\right)+\left(\mu-e^{-\jmath \theta}\right) & -\jmath\left(\mu-e^{\jmath \theta}\right)+\jmath\left(\mu-e^{-\jmath \theta}\right) \\
\jmath\left(\mu-e^{\jmath \theta}\right)-\jmath\left(\mu-e^{-\jmath \theta}\right) & \left(\mu-e^{\jmath \theta}\right)+\left(\mu-e^{-\jmath \theta}\right)
\end{array}\right] .
\end{aligned}
$$


Consequently the characteristic polynomial of $A_{i}$ can be obtained as

$$
\chi_{i}(\mu)=\left(\mu-e^{\jmath \theta}\right)\left(\mu-e^{-\jmath \theta}\right)+\frac{\lambda_{i}}{2} \varepsilon\left[\left(c_{2}+c_{1} \jmath\right)\left(\mu-e^{\jmath \theta}\right)+\left(c_{2}-c_{1} \jmath\right)\left(\mu-e^{-\jmath \theta}\right)\right] .
$$

By perturbation theory 108 it is readily seen that the two perturbed roots of (5.11) take the form of

$$
\mu_{i 1}=e^{\jmath \theta}+\mu_{i 11} \varepsilon+o(\varepsilon), \mu_{i 2}=e^{-\jmath \theta}+\mu_{i 21} \varepsilon+o(\varepsilon) .
$$

Substituting $\mu=\mu_{i 1}$ into $\chi_{i}(\mu)=0$ and comparing the coefficient of $\varepsilon$ yield

$$
\mu_{i 11}(2 \jmath \sin \theta)+\frac{1}{2} \lambda_{i}\left(c_{2}-c_{1 \jmath}\right)(2 \jmath \sin \theta)=0
$$

and $\mu_{i 11}=-\frac{1}{2} \lambda_{i}\left(c_{2}-c_{1} \jmath\right)$ follows immediately. Direct computation shows that $\left|\mu_{i 1}\right|^{2}=1+$ $2 \operatorname{Re}\left(\mu_{i 11} e^{-\jmath \theta}\right) \varepsilon+o(\varepsilon)$, where

$$
\operatorname{Re}\left(\mu_{i 11} e^{-\jmath \theta}\right)=-\frac{1}{2}\left[a_{i}\left(c_{2} \cos \theta-c_{1} \sin \theta\right)+b_{i}\left(c_{1} \cos \theta+c_{2} \sin \theta\right)\right]
$$

if we let $\lambda_{i}=a_{i}+b_{i}$ j. Clearly $\left|\mu_{i 1}\right|=1-\frac{1}{2}\left(\operatorname{Re} \lambda_{i}\right)\left(c_{2} \cos \theta-c_{1} \sin \theta\right) \varepsilon+o(\varepsilon)$ when $c_{1} \cos \theta+c_{2} \sin \theta=0$. Similarly we can show $\mu_{i 21}=-\frac{1}{2} \lambda_{i}\left(c_{2}+c_{1} \jmath\right)$ and $\left|\mu_{i 2}\right|=1-\frac{1}{2} \operatorname{Re} \lambda_{i}\left(c_{2} \cos \theta-c_{1} \sin \theta\right) \varepsilon+o(\varepsilon)$, which implies the conclusion.

2 ). Here we need to compute the Jordan decomposition of $A_{i}$. The eigenvector corresponding to the eigenvalue $\mu_{i 1}$ is given by $v_{i 1}=v_{i 10}+v_{i 11} \varepsilon+o(\varepsilon)$. Substituting it into the equation $A_{i} v_{i 1}=\mu_{i 1} v_{i 1}$ and comparing the coefficients of the constant term, we have $A v_{i 10}=e^{\jmath \theta} v_{i 10}$. With the normalization condition $v^{\prime} v_{i 1}=1$ where $v=\frac{1}{2}\left[\begin{array}{ll}1 & -\jmath\end{array}\right]^{\prime}, v_{i 10}=\left[\begin{array}{ll}1 & j\end{array}\right]^{\prime}$. Similarly, the eigenvector corresponding

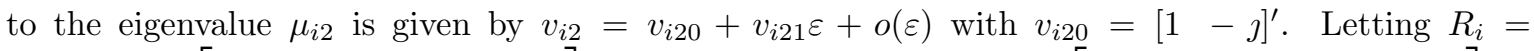
$\left(\begin{array}{ll}v_{i 1} & v_{i 2}\end{array}\right)=\left[\begin{array}{cc}1+O(\varepsilon) & 1+O(\varepsilon) \\ \jmath+O(\varepsilon) & -\jmath+O(\varepsilon)\end{array}\right]$, it is clear that $R_{i}^{-1}=\frac{1}{\operatorname{det} R_{i}}\left[\begin{array}{cc}-\jmath+O(\varepsilon) & -1+O(\varepsilon) \\ -\jmath+O(\varepsilon) & 1+O(\varepsilon)\end{array}\right]=$ $\frac{1}{2}\left[\begin{array}{cc}1+O(\varepsilon) & -\jmath+O(\varepsilon) \\ 1+O(\varepsilon) & \jmath+O(\varepsilon)\end{array}\right]$. The result follows directly by noticing that $A_{i}=R_{i}\left[\begin{array}{ll}\mu_{i 1} & \\ & \mu_{i 2}\end{array}\right] R_{i}^{-1}$.

Remark 5.3. Denote $\rho=\max _{i=2, \ldots, l} \rho_{i}$ and let $h$ be a constant in $\left(0\right.$, Re $\left.\lambda_{2}\right]$. Taking $c_{1}=-\sin \theta / h$ and $c_{2}=\cos \theta / h$, we have $\rho \leq 1-\varepsilon+o(\varepsilon)<1-\varepsilon / 2$ with sufficiently small $\varepsilon$. 
We also need to define some constants as follows:

$$
\begin{aligned}
C_{0} & =\frac{1}{2}\left|c_{1}\right|+\frac{3}{2}\left|c_{2} \csc \theta\right|=\frac{1}{2 h}(|\sin \theta|+3|\cot \theta|), \\
\Lambda & =\max _{i=2, \ldots, l}\left|\lambda_{i}\right|, \\
C(k) & = \begin{cases}\left\|T_{L}^{-1}\right\|_{\infty}+2 C_{0} \Lambda\left\|T_{L}\right\|_{\infty}, & k=1 \\
\left\|T_{L}^{-1}\right\|_{\infty}+2 C_{0}(\Lambda+1)\left\|T_{L}\right\|_{\infty}+10\left(\left|c_{1}\right|+\left|c_{2}\right|\right) C(k-1), & k \geq 2,\end{cases} \\
\bar{C} & =5\left(\left|c_{1}\right|+\left|c_{2}\right|\right) C\left(N_{\max }\right)+C_{0}\left\|T_{L}\right\|_{\infty},
\end{aligned}
$$

where $N_{\max }=\max _{i=2, \ldots, l} N_{l}$.

Lemma 5.2. Let $\gamma=1-\varepsilon / 4$. Then we can choose sufficiently small $\varepsilon$ to satisfy the following inequalities:

$$
\begin{array}{r}
(\Lambda+1) \bar{C} \varepsilon \leq \frac{1}{2} \gamma|\csc \theta|\left\|T_{L}\right\|_{\infty} \\
\frac{1}{\gamma}\left(2|\cos \theta|+\frac{1}{\gamma}\right) \leq 2|\cos \theta|+1+\frac{1}{2} \\
(N-1) \bar{C}(\Lambda+1) \varepsilon \leq \frac{1}{4}|\csc \theta| \gamma^{3}
\end{array}
$$

Theorem 5.1. Take $c_{j}$ 's as in Remark 5.3 and let $\gamma=1-\varepsilon / 4$. Select sufficiently small $\varepsilon$ to satisfy Lemmas 5.1 and 5.2 with $\rho<1-\varepsilon / 2$. Then under Assumptions 5.1 and 5.2, consensus can be achieved at a convergence rate of $O\left(\gamma^{t}\right)$ provided that $g_{0} \geq \max \left\{\frac{4}{3 \gamma} C^{*}, C_{\delta}^{*}\right\}$ and $M(t)$ satisfies

$$
\left\{\begin{array}{l}
M(t) \geq 1, t=1,2 \\
M(t) \geq|\cos \theta|+1 / 2, t=2 m+1, \ldots
\end{array}\right.
$$

Therefore, the number of sufficient bits for the consensus is $\left\lceil\log _{2} 2\lceil|\cos \theta|+1 / 2\rceil\right\rceil$.

Proof. 1) Disagreement dynamics. The closed-loop system of disagreement vectors can be established as

$$
\left[\begin{array}{c}
\delta_{1}(t+1) \\
\delta_{2}(t+1)
\end{array}\right]=\left[\begin{array}{cc}
\cos \theta I_{N} & \sin \theta I_{N} \\
-\sin \theta I_{N} & \cos \theta I_{N}
\end{array}\right]\left[\begin{array}{c}
\delta_{1}(t) \\
\delta_{2}(t)
\end{array}\right]+\left[\begin{array}{c}
0 \\
u(t)
\end{array}\right]
$$

with

$$
u(t)=\left\{\begin{array}{l}
0, t=0,1 \\
-\sum_{j=1}^{2} k_{j} L\left(\delta_{j}(t)+e_{j}(t)\right), t \geq 2
\end{array}\right.
$$

by noticing 5.6 and $L=L\left(I_{N}-\phi_{1} \psi_{1}^{\prime}\right)$. Letting $\tilde{\delta}_{j}(t)=T_{L}^{-1} \delta_{j}(t)=\left[\tilde{\delta}_{1 j}(t), \ldots, \tilde{\delta}_{N, j}(t)\right]^{\prime}$, we obtain

$$
\left[\begin{array}{c}
\tilde{\delta}_{1}(t+1) \\
\tilde{\delta}_{2}(t+1)
\end{array}\right]=\left[\begin{array}{cc}
\cos \theta I_{N} & \sin \theta I_{N} \\
-\sin \theta I_{N}-k_{1} L_{J} & \cos \theta I_{N}-k_{2} L_{J}
\end{array}\right]\left[\begin{array}{c}
\tilde{\delta}_{1}(t) \\
\tilde{\delta}_{2}(t)
\end{array}\right]+\left[\begin{array}{c}
0 \\
\eta(t)
\end{array}\right]
$$


where $\eta(t)=-L_{J} T_{L}^{-1}\left(k_{1} e_{1}(t)+k_{2} e_{2}(t)\right)$. Denote $\tilde{\delta}^{i}(t)=\left[\tilde{\delta}_{i 1}(t), \tilde{\delta}_{i 2}(t)\right]^{\prime}$ for $i=1, \ldots, N$. Clearly $\tilde{\delta}^{1}(t) \equiv 0$ due to that $\tilde{\delta}_{1 j}(t)=\psi_{1}^{\prime}\left(I_{N}-\phi_{1} \psi_{1}^{\prime}\right) x_{j}(t)=0$ for $j=1,2$. Without loss of generality we assume that $N_{2}=2$ (the Jordan block with respect to $\lambda_{2}$ is two-dimensional) and consequently $\tilde{\delta}^{2}(t)$ and $\tilde{\delta}^{3}(t)$ are coupled in the following way:

$$
\begin{aligned}
& \tilde{\delta}^{i}(t+1)=A \tilde{\delta}^{i}(t), t=0,1, i=2,3 ; \\
& \tilde{\delta}^{2}(t+1)=A_{2} \tilde{\delta}^{2}(t)-K \tilde{\delta}^{3}(t)-\eta_{2}(t), \\
& \tilde{\delta}^{3}(t+1)=A_{2} \tilde{\delta}^{3}(t)-\eta_{3}(t), t \geq 2,
\end{aligned}
$$

where $A_{2}$ and $K$ have been defined in Lemma 5.1 and $\eta_{2}(t)=\left[0,\left(\lambda_{2} \psi_{2}^{\prime}+\psi_{3}^{\prime}\right)\left(k_{1} e_{1}(t)+k_{2} e_{2}(t)\right)\right]^{\prime}$, $\eta_{3}(t)=\left[0, \lambda_{2} \psi_{3}^{\prime}\left(k_{1} e_{1}(t)+k_{2} e_{2}(t)\right)\right]^{\prime}$.

2) Estimation error and exponential convergence. Remember that $e_{2}(t)=\cot \theta e_{1}(t)-\csc \theta e_{1}(t-$ 1) $-u(t-1)$ is dependent on the control input by (5.8), we have to first make an estimate for $u(t)$ before establishing the consensus result. Below we shall show $\left|\psi_{i}^{\prime} u(t)\right| \leq \varepsilon(\Lambda+1) \bar{C} p_{0} \gamma^{t-2}, t \geq 2$ for $i \geq 2$ by induction.

With the choice of $p_{0}$ and $\gamma$ it is easy to see $\left|s_{i}(t)\right| \leq 3 / 2$ when $t \leq 2$ by noticing $\left|y_{i}(t)\right| \leq 2 C^{*}$, hence we obtain $\max _{t=1,2}\|\Delta(t)\|_{\infty} \leq 1 / 2$ if $M(1), M(2) \geq 1$. For $i=2$, we have $\psi_{2}^{\prime} L=\lambda_{2} \psi_{2}^{\prime}+\psi_{3}^{\prime}$ and as a result

$$
\begin{aligned}
\left|\psi_{2}^{\prime} u(2)\right| & =\left|\psi_{2}^{\prime} L \sum_{j=1}^{2} k_{j}\left(\delta_{j}(2)+e_{j}(2)\right)\right| \\
& \leq \varepsilon(\Lambda+1)\left\|T_{L}\right\|_{\infty}\left[\left|c_{1}\right|\left(2\|\delta(0)\|_{\infty}+p(1)\|\Delta(2)\|_{\infty}\right)\right. \\
& \left.+\left|c_{2}\right|\left(2\|\delta(0)\|_{\infty}+|\cot \theta| p(1)\|\Delta(2)\|_{\infty}+|\csc \theta| p(0)\|\Delta(1)\|_{\infty}\right)\right] \\
& \leq \varepsilon(\Lambda+1)\left\|T_{L}\right\|_{\infty}\left(\left|c_{1}\right|+\left|c_{2}\right|\right)\left(2\|\delta(0)\|_{\infty}+|\csc \theta| p_{0}\right) \\
& \leq \varepsilon(\Lambda+1)\left\|T_{L}\right\|_{\infty}\left(\left|c_{1}\right|+\left|c_{2}\right|\right)(2+|\csc \theta|) p_{0} \\
& \leq \varepsilon(\Lambda+1) \bar{C} p_{0},
\end{aligned}
$$

which also holds for $\left|\psi_{i}^{\prime} u(2)\right|$ for $i>2$.

Now assume that

$$
\begin{aligned}
& \left|\psi_{i}^{\prime} u(t)\right| \leq \varepsilon(\Lambda+1) \bar{C} p_{0} \gamma^{t-2}, t \geq 2 \\
& \|\Delta(\tau)\|_{\infty} \leq 1 / 2\left(\Rightarrow\left|e_{i 1}(\tau)\right| \leq \frac{1}{2} p_{0} \gamma^{\tau-1}\right), 1 \leq \tau \leq t .
\end{aligned}
$$

Then by combining (5.18) and 5.14a it follows that

$$
\begin{aligned}
\left\|\eta_{3}(\tau)\right\|_{\infty} & =\left|\lambda_{2} \| \psi_{3}^{\prime}\left[k_{1} e_{1}(\tau)+k_{2} e_{2}(\tau)\right]\right| \\
& \leq \varepsilon\left|\lambda_{2}\right| p_{0} \gamma^{\tau-2}\left\|T_{L}\right\|_{\infty}\left(\frac{1}{2}\left|c_{1}\right|+\left|c_{2} \| \csc \theta\right|\right)+\varepsilon\left|\lambda_{2}\right| c_{2} \| \psi_{3}^{\prime} u(\tau-1) \mid \\
& \leq \varepsilon\left|\lambda_{2}\right|\left\|T_{L}\right\|_{\infty} C_{0} p_{0} \gamma^{\tau-2} .
\end{aligned}
$$


Recalling (5.17) we get that for $t \geq 2$

$$
\tilde{\delta}^{3}(t+1)=A_{2}^{t-1} \tilde{\delta}^{3}(2)-\sum_{\tau=1}^{t-1} A_{2}^{t-1-\tau} \eta_{3}(\tau+1),
$$

which produces the following estimate by Lemma 5.1 and 5.19

$$
\begin{aligned}
\left\|\tilde{\delta}^{3}(t+1)\right\|_{\infty} & \leq \frac{5}{2}\left(\rho_{2}^{t-1}\left\|\tilde{\delta}^{3}(2)\right\|_{\infty}+4\left|\lambda_{2}\right| C_{0}\left\|T_{L}\right\|_{\infty} p_{0} \gamma^{t-1}\right) \\
& \leq 5 \gamma^{t-1}\left(\left\|T_{L}^{-1}\right\|_{\infty}\|\delta(0)\|_{\infty}+2\left|\lambda_{2}\right| C_{0}\left\|T_{L}\right\|_{\infty} p_{0}\right) \\
& \leq 5 C(1) p_{0} \gamma^{t-1} .
\end{aligned}
$$

Similarly, an estimate for $\left\|\tilde{\delta}_{2}(t+1)\right\|_{\infty}$ can be found as $\left\|\tilde{\delta}^{2}(t+1)\right\|_{\infty} \leq 5 C(2) p_{0} \gamma^{t-1}$, if we notice that

$$
\begin{aligned}
\left\|K \tilde{\delta}^{3}(\tau)\right\|_{\infty} & \leq 5 \varepsilon\left(\left|c_{1}\right|+\left|c_{2}\right|\right) C(1) p_{0} \gamma^{\tau-2} \\
\left\|\eta_{2}(\tau)\right\|_{\infty} & \leq \varepsilon\left(\left|\lambda_{2}\right|+1\right)\left\|T_{L}\right\|_{\infty} C_{0} p_{0} \gamma^{\tau-2}, 2 \leq \tau \leq t
\end{aligned}
$$

For any $i \geq 2$, by proceeding along the same line as in the above it is concluded that

$$
\left\|\tilde{\delta}^{i}(t+1)\right\|_{\infty} \leq 5 C\left(N_{\max }\right) p_{0} \gamma^{t-1}
$$

3) Data rate. Now we are able to discuss the estimation for $\left|\psi_{i}^{\prime} u(t+1)\right|$, which is bounded by the sum of $\left|\sum_{j=1}^{2} k_{j} \psi_{i}^{\prime} L \delta_{j}(t+1)\right|$ and $\left|\sum_{j=1}^{2} k_{j} \psi_{i}^{\prime} L e_{j}(t+1)\right|$. For the first term, by [5.21) it is readily seen that

$$
\begin{aligned}
\left|\sum_{j=1}^{2} k_{j} \psi_{i}^{\prime} L \delta_{j}(t+1)\right| & \leq\left|\sum_{j=1}^{2} k_{j} \psi_{i}^{\prime} T_{L} L_{J} \tilde{\delta}_{j}(t+1)\right| \\
& \leq 5 \varepsilon\left(\left|c_{1}\right|+\left|c_{2}\right|\right) C\left(N_{\max }\right) p_{0} \gamma^{t-1}
\end{aligned}
$$

while the second term is essentially related with $e_{j}(t+1)$, or more exactly $\Delta(t+1)$. By $(5.7)$ and (5.8) we have

$$
\begin{aligned}
d(t+1) & =\frac{1}{p(t)}\left[-\cos \theta e_{1}(t)-\sin \theta e_{2}(t)\right] \\
& =\frac{-2 \cos \theta}{\gamma} \Delta(t)+\frac{1}{\gamma^{2}} \Delta(t-1)+\frac{1}{p(t)} \sin \theta u(t-1),
\end{aligned}
$$

which is obviously dependent on the previous quantization errors $\Delta(t)$ and $\Delta(t-1)$, as well as the previous control input $u(t-1)$. Hence with the induction assumption (5.18) the quantizer can be made unsaturated with sufficiently many bits at time $t+1$, and $\|\Delta(t+1)\|_{\infty} \leq 1 / 2$ follows directly. Consequently

$$
\left|\sum_{j=1}^{2} k_{j} \psi_{i}^{\prime} L e_{j}(t+1)\right| \leq \varepsilon\left|\lambda_{2}\right|\left\|T_{L}\right\|_{\infty} C_{0} p_{0} \gamma^{t-1}
$$


as in 5.19). The induction is then established by combining 5.22. Moreover, by 5.21 the consensus can be achieved at a convergence rate of $O\left(\gamma^{t}\right)$.

Below we are to calculate the number of required quantization levels at each time step. The situation when $t \leq 2$ has been discussed. When $t>2$, from 5.23 we can see that

$$
\begin{aligned}
\|d(t)\|_{\infty} & \leq \frac{1}{2 \gamma}\left(2|\cos \theta|+\frac{1}{\gamma}\right)+\frac{|\sin \theta|}{p(t-1)}\|u(t-2)\|_{\infty} \\
& \leq \frac{1}{2}(2|\cos \theta|+1)+\frac{1}{4}+\frac{|\sin \theta|}{\gamma^{3}}(N-1)(\Lambda+1) \bar{C} \\
& \leq \frac{1}{2}(2|\cos \theta|+1)+\frac{1}{2}
\end{aligned}
$$

by noticing 5.14b, 5.14c and $u(t)=\sum_{i=2}^{N} \phi_{i} \psi_{i}^{\prime} u(t)\left(\psi_{1}^{\prime} u(t)=0\right)$. In summary, the proof is completed.

Remark 5.4. For the coupling system shown in (5.17), we divide it into two subsystems with disturbance. Each subsystem can be stabilized as long as the disturbance decays exponentially at a speed slower than $\rho_{2}$, i.e. $\left\|\eta_{3}(t)\right\|_{\infty}=O\left(\gamma^{t}\right)$ and $\left\|K \tilde{\delta}^{3}(t)+\eta_{2}(t)\right\|_{\infty}=O\left(\gamma^{t}\right)$, with $\rho_{2}<\gamma<1$. The interference of $u(t)$ in the estimation error $e(t)$ can be ignored, as long as $\|u(t)\|_{\infty}=O\left(\varepsilon^{\alpha}\right) p_{0} \gamma^{t}$ with $\alpha>0$, yielding that $\left\|\eta_{3}(t)\right\|_{\infty}=O(\varepsilon) p_{0} \gamma^{t}$, and then $\left\|\tilde{\delta}^{3}(t)\right\|_{\infty}=O\left(\gamma^{t}\right)$ follows. As a result, $\left\|K \tilde{\delta}^{3}(t)\right\|_{\infty}=O(\varepsilon) \gamma^{t}$ and $\left\|\tilde{\delta}^{3}(t)\right\|_{\infty}=O\left(\gamma^{t}\right)$ follow by combining $\left\|\eta_{3}(t)\right\|_{\infty}=O(\varepsilon) p_{0} \gamma^{t}$. Such a reasoning still applies when (5.17) involves more than two subsystems. Finally we show that $\|u(t)\|_{\infty}=O(\varepsilon) p_{0} \gamma^{t}$, and by 5.23) we conclude that the control input does not consume extra bits in exchanging the information when the control gains are sufficiently small.

\subsection{Higher-order Cases}

In this section, we will carry out the same task as in the last section for general higher-order cases. The analysis actually proceeds along a similar line, but the assignment of control gains to achieve consensus is much more challenging, and we have to resort to combinatorial identities for an explicit data rate. As before, we first provide an encoding-decoding scheme for all the agents and devise a control protocol in terms of the outputs of the encoder/decoder. Then we present some lemmas, which will play a crucial role in the convergence analysis and the derivation of the data rate in the final part.

\subsubsection{Encoding-decoding Scheme and Distributed Control Law}

As pointed out in the last section, the construction of the encoding scheme should follow two principles: firstly, the encoder is able to estimate other state variables given that only the first component is 
measurable; secondly, the estimation should be based on iterations in an effort to reduce quantization levels. Such an idea can be stated more clearly as follows. At each time step, the scaled difference between the output $y_{i}(t)$ and its estimate is quantized to obtain a signal $s_{i}(t)$. Based on $s_{i}(t)$ we construct an estimate $\hat{x}_{i 1}(t)$ of the first component $x_{i 1}(t)$, and combine previous estimates $\hat{x}_{i 1}(t-1)$ through $\hat{x}_{i 1}(t-2 m+1)$ to obtain estimates of the other components $x_{i 2}(t)$ through $x_{i, 2 m}(t)$.

To be detailed, denote the observability matrix $\mathcal{O}=\left[\begin{array}{c}I_{2 m}(1, \cdot) \\ A(1, \cdot) \\ \vdots \\ A^{2 m-1}(1, \cdot)\end{array}\right]$,

$$
\begin{aligned}
\bar{x}_{i}(t) & =\left[x_{i 1}(t-2 m+1), x_{i 1}(t-2 m+2), \ldots, x_{i 1}(t)\right]^{\prime}, \\
b_{n}(\theta) & =\left[0, \ldots, 0, A(1,2 m), \ldots, A^{n-1}(1,2 m)\right]^{\prime} \in \mathbb{R}^{2 m} .
\end{aligned}
$$

We have

$$
\bar{x}_{i}(t)=\mathcal{O} x^{i}(t-2 m+1)+\sum_{n=1}^{2 m-1} b_{n}(\theta) u_{i}(t-n),
$$

if we notice by (5.1) that for $k=1, \ldots, 2 m$,

$$
x^{i}(t-2 m+k)=A^{k-1} x^{i}(t-2 m+1)+\sum_{n=0}^{k-2} A^{k-2-n} b u_{i}(t-2 m+1+n) .
$$

As a result, it follows that

$$
x^{i}(t-2 m+1)=\mathcal{O}^{-1}\left[\bar{x}_{i}(t)-\sum_{n=1}^{2 m-1} b_{n}(\theta) u_{i}(t-n)\right]
$$

and

$$
\begin{aligned}
x^{i}(t) & =A^{2 m-1} x^{i}(t-2 m+1)+\sum_{j=0}^{2 m-2} A^{2 m-2-j} b u_{i}(t-2 m+1+j) \\
& =S \bar{x}_{i}(t)+\sum_{j=1}^{2 m-1} \tilde{b}_{j}(\theta) u_{i}(t-j),
\end{aligned}
$$

where $S=A^{2 m-1} \mathcal{O}^{-1}$ (the existence of $\mathcal{O}^{-1}$ can be easily verified by PBH test 113 if $\left.\sin \theta \neq 0\right)$ and $\tilde{b}_{n}(\theta)=-S b_{n}(\theta)+A^{n-1}(\cdot, 2 m)$. Inspired by (5.28), the encoding scheme for agent $i$ is implemented below:

for $t \leq 2 m$,

$$
\left\{\begin{array}{c}
s_{i}(t)=q_{t}\left(\frac{y_{i}(t)}{p(t-1)}\right), \hat{x}_{i 1}(t)=p(t-1) s_{i}(t) \\
{\left[\begin{array}{c}
\hat{x}_{i 2}(2 m) \\
\vdots \\
\hat{x}_{i, 2 m}(2 m)
\end{array}\right]=S_{m}\left[\begin{array}{c}
\hat{x}_{i 1}(1) \\
\vdots \\
\hat{x}_{i 1}(2 m)
\end{array}\right]}
\end{array}\right.
$$


for $t>2 m$,

$$
\left\{\begin{array}{l}
s_{i}(t)=q_{t}\left(\frac{y_{i}(t)-\left[\cos \theta \hat{x}_{i 1}(t-1)+\sin \theta \hat{x}_{i 2}(t-1)+\hat{x}_{i 3}(t-1)\right]}{p(t-1)}\right) \\
\hat{x}_{i 1}(t)=\cos \theta \hat{x}_{i 1}(t-1)+\sin \theta \hat{x}_{i 2}(t-1)+\hat{x}_{i 3}(t-1)+p(t-1) s_{i}(t) \\
{\left[\begin{array}{c}
\hat{x}_{i 2}(t) \\
\vdots \\
\hat{x}_{i, 2 m}(t)
\end{array}\right]=S_{m}\left[\begin{array}{c}
\hat{x}_{i 1}(t-2 m+1) \\
\vdots \\
\hat{x}_{i 1}(t)
\end{array}\right]}
\end{array}\right.
$$

where $S_{m}=S(2: 2 m, \cdot)$ is a submatrix of $S$ obtained by deleting the first row, and $p(t)=p_{0} \gamma^{t}, 0<$ $\gamma<1$ is a decaying scaling function.

After $s_{i}(t)$ is generated, transmitted and received by one of agent $i$ 's out-neighbors, say $v \in \mathcal{N}_{i}^{-}$, a decoder will be activated:

for $t \leq 2 m$,

$$
\left\{\begin{array}{l}
\hat{x}_{i v 1}(t)=p(t-1) s_{i}(t) \\
{\left[\begin{array}{c}
\hat{x}_{i v 2}(2 m) \\
\vdots \\
\hat{x}_{i v, 2 m}(2 m)
\end{array}\right]=S_{m}\left[\begin{array}{c}
\hat{x}_{i v 1}(1) \\
\vdots \\
\hat{x}_{i v 1}(2 m)
\end{array}\right]}
\end{array}\right.
$$

for $t>2 m$,

$$
\left\{\begin{array}{l}
\hat{x}_{i v 1}(t)=\cos \theta \hat{x}_{i v 1}(t-1)+\sin \theta \hat{x}_{i v 2}(t-1)+\hat{x}_{i v 3}(t-1)+p(t-1) s_{i}(t) \\
{\left[\begin{array}{c}
\hat{x}_{i v 2}(t) \\
\vdots \\
\hat{x}_{i v, 2 m}(t)
\end{array}\right]=S_{m}\left[\begin{array}{c}
\hat{x}_{i v 1}(t-2 m+1) \\
\vdots \\
\hat{x}_{i v 1}(t)
\end{array}\right]}
\end{array}\right.
$$

Remark 5.5. Comparing 5.29) with 5.31, 5.30) with (5.32), it is clear that $\hat{x}_{i v j}(t) \equiv \hat{x}_{i j}(t), j=$ $1, \ldots, 2 m$, for $v \in \mathcal{N}_{i}^{-}, i=1, \ldots, N$. Denote $e_{i j}(t)=\hat{x}_{i j}(t)-x_{i j}(t)$ as the estimation error, $\Delta_{i}(t)=$ $s_{i}(t)-d_{i}(t)$ as the quantization error, where

$$
d_{i}(t)=\left\{\begin{array}{l}
\frac{y_{i}(t)}{p(t-1)}, t=1,2, \ldots, 2 m \\
\frac{y_{i}(t)-\left[\cos \theta \hat{x}_{i 1}(t-1)+\sin \theta \hat{x}_{i 2}(t-1)+\hat{x}_{i 3}(t-1)\right]}{p(t-1)}, t>2 m .
\end{array}\right.
$$

Comparing (5.28) with 5.30, the estimation errors are given by the following:

$$
\left\{\begin{aligned}
e_{i 1}(t) & =p(t-1) \Delta_{i}(t), t \geq 1 \\
e_{i j}(t) & =\sum_{n=1}^{2 m} S(j, n) e_{i 1}(t-2 m+n)-\sum_{n=1}^{2 m-1} \tilde{b}_{n j}(\theta) u_{i}(t-n) \\
t & \geq 2 m, j=2, \ldots, 2 m
\end{aligned}\right.
$$


where $\tilde{b}_{n j}$ is the $j$-th entry of $\tilde{b}_{n}$.

Remark 5.6. The encoding schemes (5.4) and (5.30) are quite different from those in [114] or Chapter 4. In more detail, to address the general dynamics with unmeasurable states, [114] designed an encoding scheme respectively for the output and control input, and used a Luenberger observer to estimate the unmeasurable states. If we compare with the integrator case studied in Chapter 4, we can also see a big difference: the special structure of $n$-th order integrator dynamics enables it to easily "recover" the control input at $n$ steps earlier, based on which an estimate of the unmeasurable components can be made with time delay, and the encoding scheme can be designed accordingly. However, for oscillator systems it is unlikely to do the same and we resort to the constructability of the system, namely we estimate the unmeasurable states directly from $\hat{x}_{i 1}(t)$ through $\hat{x}_{i 1}(t-2 m+1)$. Although such a method introduces the control input into the estimation errors, it is able to make an estimation without time delay, and hence avoids the stabilization of a time-delayed closed-loop system in the consensus analysis.

For agent $i$, the outputs of encoder are $\hat{x}_{i 1}(t), \ldots, \hat{x}_{i, 2 m}(t)$, while the outputs of decoders are $\hat{x}_{v i 1}(t), \ldots, \hat{x}_{v i, 2 m}(t)$ for $v \in \mathcal{N}_{i}^{+}$. Based on these outputs, the distributed control law of agent $i$ is proposed as

$$
u_{i}(t)=\left\{\begin{array}{l}
0, t=0,1, \ldots, 2 m-1 \\
\sum_{j=1}^{2 m} k_{j} \sum_{l \in \mathcal{N}_{i}^{+}} w_{i l}\left[\hat{x}_{l i j}(t)-\hat{x}_{i j}(t)\right], t \geq 2 m .
\end{array}\right.
$$

\subsubsection{Lemmas}

The following two lemmas are respectively needed in analyzing consensus and data rate. The first one is to stabilize the closed-loop system of disagreements, and the second one is used for estimating the magnitude of $u_{i}(t)$ and $d_{i}(t)$.

Lemma 5.3. Denote $A_{i}=A-\lambda_{i} K$ with $\lambda_{i}>0$, where $K \in \mathbb{R}^{2 m \times 2 m}$ and its nonzero entries are only at the last row $\left[k_{1}, k_{2}, \ldots, k_{2 m-1}, k_{2 m}\right]$. Take

$$
\begin{aligned}
k_{2 j-1}=\left\{\begin{array}{l}
c_{2 j-1} \varepsilon^{m-j}, j=1, \ldots, m-1 ; \\
c_{2 j-1} \varepsilon, j=m,
\end{array}\right. \\
k_{2 j}=\left\{\begin{array}{l}
c_{2 j} \varepsilon^{m-j}, j=1, \ldots, m-1 ; \\
c_{2 j} \varepsilon, j=m .
\end{array}\right.
\end{aligned}
$$

Then we can find constants $c_{2 j-1}$ and $c_{2 j}(j=1, \ldots, m)$ such that, when $\varepsilon$ is sufficiently small, the spectral radius $\rho_{i}$ of $A_{i}$ is less than 1 with distinct eigenvalues. Moreover, denote

$$
R_{m}=\frac{1}{2}+\frac{1}{2}\left(c_{2 m-1} \sin \theta-c_{2 m} \cos \theta\right), H=R e\left(\frac{c_{2 m-5} \jmath-c_{2 m-4}}{c_{2 m-3} \jmath-c_{2 m-2}} e^{-\jmath \theta}\right)
$$


The requirements about $c_{2 j-1}$ 's and $c_{2 j}$ 's corresponding to different $m$ 's are listed below.

1). $m=2$ : let $c_{1}=-\sin 2 \theta$ and $c_{2}=\cos 2 \theta$. If $R_{2}<0$, then $\rho_{i}=1+\frac{1}{2} \lambda_{i} R_{2} \varepsilon+o(\varepsilon)$;

2). $m \geq 3$ : let $c_{2 m-3}=-\sin 2 \theta$ and $c_{2 m-2}=\cos 2 \theta$. If $\lambda_{i} R_{m}+H<0$ and $\operatorname{Re}\left(\vartheta_{n 1} e^{-\jmath \theta}\right)<0$ with $\vartheta_{n 1}, n=3, \ldots, m$ denoting the $m-2$ distinct roots of the equation

$$
\vartheta_{1}^{m-2}\left(c_{2 m-2}-c_{2 m-3} \jmath\right)+\cdots+\vartheta_{1}\left(c_{4}-c_{3} \jmath\right)+\left(c_{2}-c_{1} \jmath\right)=0,
$$

then $\rho_{i}=1+\frac{1}{2} \max _{n=3, \ldots, m}\left\{\lambda_{i} R_{m}+H, 2 \operatorname{Re}\left(\vartheta_{n 1} e^{-\jmath \theta}\right)\right\} \varepsilon+o(\varepsilon)$.

Lemma 5.4. Assume that Lemma 5.3 holds. When $\varepsilon$ is sufficiently small, for any vector $\xi \in \mathbb{R}^{2 m}$, the entries of $A_{i}^{t} \xi, t \in \mathbb{N}^{+}$(denoted as $\xi_{t, 2 j-1}^{i}$ and $\xi_{t, 2 j}^{i}, j=1, \ldots, m$ ) satisfy that

$$
\left|\xi_{t, 2 j-1}^{i}\right|,\left|\xi_{t, 2 j}^{i}\right| \leq\left\{\begin{array}{l}
\|\xi\|_{\infty} M_{i j} \rho_{i}^{t} \varepsilon^{j-(m-1)}, j \leq m-2 \\
\|\xi\|_{\infty} M_{i j} \rho_{i}^{t} \varepsilon^{(j-m) / 2}, j=m-1, m
\end{array}\right.
$$

where

$$
M_{i j}= \begin{cases}\frac{5}{2 \lambda_{i}}\left(\sum_{n=3}^{m} \frac{\left|\vartheta_{n 1}\right|^{j-1}}{\prod_{3 \leq k \leq m, k \neq n}\left|\vartheta_{k 1}-\vartheta_{n 1}\right|}\right), & j=1, \ldots, m-3 ; \\ \frac{5}{2 \lambda_{i}}\left(\sum_{n=3}^{m} \frac{\left|\vartheta_{n 1}\right|^{m-3}}{\prod_{3 \leq k \leq m, k \neq n}\left|\vartheta_{k 1}-\vartheta_{n 1}\right|}+1\right), & j=m-2 ; \\ \frac{3}{\sqrt{2 \lambda_{i}}}, j=m-1 \\ 5 / 2, j=m .\end{cases}
$$

Remark 5.7. The proofs of the above lemmas can be found in Section 5.6. As with the integrator case, the basic idea is to combine the bifurcation analysis of the roots of characteristic polynomials and the Jordan basis of a perturbed matrix [109]. However, the situation here is much different. On the one hand, the complex conjugate eigenvalues of the original matrix A complicates the analysis of the perturbed eigenvalues, as seen from the proof of Lemma 5.3. On the other hand, unlike Chapter 4 where the unperturbed matrix admits multiple eigenvalues of 0 and 1, the unperturbed matrix here admits eigenvalues of $m$ identical pairs of complex conjugate numbers, which allows a less cumbersome calculation of the perturbed Jordan basis, as can be seen in the proof of Lemma 5.4.

Remark 5.8. Assume $0<\lambda_{2} \leq \cdots \leq \lambda_{N}$ and let $\rho=\max _{i=2, \ldots, N} \rho_{i}, h \in\left(0, \lambda_{2}\right]$. Given $c_{2 m-3}=$ $-\sin 2 \theta$ and $c_{2 m-2}=\cos 2 \theta$, the other constants $c_{2 j-1}$ and $c_{2 j}$ can be selected as follows such that $\rho \leq 1-\varepsilon+o(\varepsilon)<1-\varepsilon / 2$ holds with sufficiently small $\varepsilon$ :

1). $m=2:$ select $c_{3}=-(4 / h+1) \sin \theta, c_{4}=(4 / h+1) \cos \theta$ such that $R_{2}=-2 / h<0$;

2). $m \geq 3$ : first select $c_{2 m-4}, c_{2 m-5}, \cdots, c_{1}$ such that the solutions of (5.38) are given by $\vartheta_{n 1}=-(n-2) e^{\jmath \theta}, n=3, \ldots, m$ and $H$ is determined by (5.37). In fact, direct computation shows 
that $c_{2 m-4}-c_{2 m-5} \jmath=\frac{1}{2}(m-1)(m-2) e^{\jmath 3 \theta}$ and consequently $H=\frac{1}{2}(m-1)(m-2)>0$. Now let $c_{2 m-1}=-[(2 H+4) / h+1] \sin \theta, c_{2 m}=[(2 H+4) / h+1] \cos \theta$ such that

$$
\lambda_{i} R_{m}+H=H\left(1-\lambda_{i} / h\right)-2 \lambda_{i} / h<-2 \lambda_{i} / h \leq-2 .
$$

With such a selection, $M_{i j}=\frac{5}{2 \lambda_{i}}\left(\sum_{n=1}^{m-2} \frac{n^{j-1}}{\prod_{1 \leq k \leq m-2, k \neq n}|k-n|}\right), j=1, \ldots, m-3$ and $M_{i, m-2}=\frac{5}{2 \lambda_{i}}\left(\sum_{n=1}^{m-2} \frac{n^{m-3}}{\prod_{1 \leq k \leq m-2, k \neq n}}\right.$ 1).

To explicitly express the data rate, another lemma is required.

Lemma 5.5. Denote

$$
\pi(\theta)=\left[\pi_{0}(\theta), \pi_{1}(\theta), \ldots, \pi_{2 m-2}(\theta), \pi_{2 m-1}(\theta)\right]=\cos \theta S(1, \cdot)+\sin \theta S(2, \cdot)+S(3, \cdot) .
$$

Then for $k=0,1, \ldots, 2 m-1$, it holds that

$$
\pi_{k}(\theta)=(-1)^{k-1} \sum_{n=0}^{\lfloor k / 2\rfloor} C(m, k-2 n) C(m-(k-2 n), n)(2 \cos \theta)^{k-2 n}
$$

Moreover, $\sum_{k=0}^{2 m-1}\left|\pi_{k}(\theta)\right|=[2(1+|\cos \theta|)]^{m}-1$.

The proof of Lemma 5.5 can be found in Section 5.6. The idea is simple and direct: by regarding $\pi(\theta)$ as the solution to an equation, we are left to its verification when $\pi(\theta)$ takes the form in the lemma. Still, the computation is quite complicated and needs special techniques from combinatorics [115, 116].

\subsubsection{Convergence Analysis and Data Rate}

The notations in 5.9 will still be used, and we make the following assumptions for the subsequent analysis.

Assumption 5.3. The communication graph $\mathcal{G}$ is undirected and connected.

Assumption 5.4. There exist known positive constants $C^{*}$ and $C_{\delta}^{*}$ such that $\max _{j=1, \ldots, 2 m}\left\|x_{j}(0)\right\|_{\infty} \leq C^{*}$ and $\max _{j=1, \ldots, 2 m}\left\|\delta_{j}(0)\right\|_{\infty} \leq C_{\delta}^{*}$.

Remark 5.9. Under Assumption 5.3, $L$ is symmetric and its eigenvalues can be rearranged as $0=$ $\lambda_{1}<\lambda_{2} \leq \cdots \leq \lambda_{N}$. Moreover, for the disagreement vector $\delta_{j}(t)$ defined in $(5.9)$, we have $\psi_{1}=$ $\phi_{1}=\frac{1}{\sqrt{N}} \mathbf{1}_{N}$. The reason that we only consider the undirected graph will be clarified in Remark 5.11. Assumption 5.4 serves the same purpose as Assumption 5.2. 
We also need the following constants:

$$
\begin{aligned}
& b^{*}=\max _{l, j}\left\{\left|\tilde{b}_{l j}\right|\right\}, c^{*}=\max _{1 \leq j \leq 2 m}\left\{\left|c_{j}\right|\right\}, \\
& \Lambda_{i}=\max \left\{\lambda_{i}^{1 / 2}, \lambda_{i}^{3 / 2}\right\}, \quad \bar{C}=\frac{9}{\sqrt{2}}\left[\left\|T_{L}^{-1}\right\|_{\infty}+5 c^{*} m N\left(\|S\|_{\infty}+2\right)\right] .
\end{aligned}
$$

Lemma 5.6. Let $\gamma=1-\varepsilon / 4$. Then we can find sufficiently small $\varepsilon$ to satisfy the following inequalities:

$$
\begin{gathered}
2 c^{*} \sum_{j \neq m-1} M_{i j} \varepsilon^{1 / 2} \leq M_{i, m-1}, i=2, \ldots, N \\
\frac{1}{\gamma^{2 m}} \sum_{k=0}^{2 m-1}\left|\pi_{k}(\theta)\right| \leq[2(1+|\cos \theta|)]^{m}-\frac{1}{2} \\
\quad(2 m-1) b^{*}(N-1) \Lambda \bar{C} \varepsilon^{1 / 2} \leq \frac{1}{8} \gamma^{4 m-1},
\end{gathered}
$$

where $\Lambda=\max _{i} \Lambda_{i}$.

Theorem 5.2. Take $k_{j}$ 's as in (5.36), $c_{j}$ 's as in Remark 5.8 and $\gamma=1-\varepsilon / 4$. Select sufficiently small $\varepsilon$ to satisfy Lemma 5.6 and $\rho_{i}<1-\varepsilon / 2$ for $i=2, \ldots, N$. Then under Assumptions 5.3 and 5.4. consensus can be achieved at a convergence rate of $O\left(\gamma^{t}\right)$ provided that $M(t)$ satisfies

$$
\left\{\begin{array}{l}
M(t) \geq 1, t=1, \ldots, 2 m \\
M(t) \geq 2^{m-1}(1+|\cos \theta|)^{m}-\frac{1}{2}, t=2 m+1, \ldots
\end{array}\right.
$$

and $p_{0} \geq(\sqrt{2}+1)^{2 m} \max \left\{C^{*}, C_{\delta}^{*}\right\}$.

Therefore, we can use $\left\lceil\log _{2} 2\left\lceil 2^{m-1}(1+|\cos \theta|)^{m}-\frac{1}{2}\right\rceil\right\rceil$ bits of information exchange to achieve the consensus.

Proof. 1) Preparation. By (5.35) we have

$$
u(t)=\left\{\begin{array}{l}
0, t=0,1, \ldots, 2 m-1 \\
-\sum_{j=1}^{2 m} k_{j} L\left(\delta_{j}(t)+e_{j}(t)\right), t \geq 2 m .
\end{array}\right.
$$

Direct computation shows

$$
\left[\begin{array}{c}
\delta_{1}(t+1) \\
\vdots \\
\delta_{2 m}(t+1)
\end{array}\right]=\left(A \otimes I_{N}\right)\left[\begin{array}{c}
\delta_{1}(t) \\
\vdots \\
\delta_{2 m}(t)
\end{array}\right]+\left[\begin{array}{c}
0 \\
\vdots \\
u(t)
\end{array}\right]
$$


Let $\tilde{\delta}_{j}(t)=T_{L}^{-1} \delta_{j}(t)=\left[\tilde{\delta}_{1 j}(t), \ldots, \tilde{\delta}_{N, j}(t)\right]^{\prime}$ and $\tilde{\delta}^{i}(t)=\left[\tilde{\delta}_{i 1}(t), \ldots, \tilde{\delta}_{i, 2 m}(t)\right]^{\prime}$. Then we obtain $\tilde{\delta}^{1}(t) \equiv$ 0 , and for $i=2, \ldots, N$

$$
\tilde{\delta}^{i}(t+1)= \begin{cases}A \tilde{\delta}^{i}(t), & t=0,1 \ldots, 2 m-1 \\ A_{i} \tilde{\delta}^{i}(t)-\epsilon_{i}(t), & t \geq 2 m\end{cases}
$$

where $\epsilon_{i}(t)=\left[0, \ldots, 0, \sum_{j=1}^{2 m} k_{j} \lambda_{i} \phi_{i}^{\prime} e_{j}(t)\right]^{\prime} \in \mathbb{R}^{2 m}$.

2) Estimation error and exponential convergence. To analyze the influence of $u(t)$ on the error term $e_{j}(t)$, we will show $\left|\phi_{i}^{\prime} u(t)\right| \leq \Lambda_{i} \bar{C} p_{0} \gamma^{t-2 m} \varepsilon^{1 / 2}, t \geq 2 m$ by induction.

With the choice of $p_{0}$ and $\gamma$ it's easy to see $\left|s_{i}(t)\right| \leq 3 / 2$ when $t \leq 2 m$ by noticing $\left|y_{i}(t)\right| \leq$ $(\sqrt{2}+1)^{2 m} C^{*}$, hence we obtain $\max _{1 \leq t \leq 2 m}\|\Delta(t)\|_{\infty} \leq 1 / 2$ by $M(t) \geq 1, t=1, \ldots, 2 m$. Moreover, $\left\|\delta_{j}(2 m)\right\|_{\infty} \leq(\sqrt{2}+1)^{2 m} C_{\delta}^{*}$. By recalling 5.35 and $\left\|e_{j}(2 m)\right\|_{\infty} \leq p_{0}\|S\|_{\infty} \max _{1 \leq t \leq 2 m}\|\Delta(t)\|_{\infty}$, we have

$$
\begin{aligned}
\left|\phi_{i}^{\prime} u(2 m)\right| & =\left|\phi_{i}^{\prime} \sum_{j=1}^{2 m} k_{j} L\left(\delta_{j}(2 m)+e_{j}(2 m)\right)\right| \\
& \leq 2 \lambda_{i} m N c^{*} \varepsilon\left[(\sqrt{2}+1)^{2 m} C_{\delta}^{*}+\frac{1}{2} p_{0}\|S\|_{\infty}\right] \\
& \leq 2 \lambda_{i} m N c^{*} \varepsilon\left(1+\frac{1}{2}\|S\|_{\infty}\right) p_{0} \\
& \leq \Lambda_{i} \bar{C} p_{0} \varepsilon^{1 / 2}
\end{aligned}
$$

by noticing that $p_{0} \geq(\sqrt{2}+1)^{2 m} C_{\delta}^{*}$ and $\varepsilon<1$.

Assume that

$$
\begin{aligned}
\left|\phi_{i}^{\prime} u(\tau)\right| & \leq \Lambda_{i} \bar{C} p_{0} \gamma^{\tau-2 m} \varepsilon^{1 / 2}, 2 m \leq \tau \leq t \\
\|\Delta(\tau)\|_{\infty} & \leq \frac{1}{2}\left(\Rightarrow\left|e_{i 1}(\tau)\right| \leq \frac{1}{2} p_{0} \gamma^{\tau-1}\right), 1 \leq \tau \leq t .
\end{aligned}
$$

Noticing that

$$
e_{j}(\tau)=\left[\begin{array}{ccc}
e_{11}(\tau-2 m+1) & \ldots & e_{11}(\tau) \\
\vdots & \ddots & \vdots \\
e_{N 1}(\tau-2 m+1) & \ldots & e_{N 1}(\tau)
\end{array}\right]\left[\begin{array}{c}
S(j, 1) \\
\vdots \\
S(j, 2 m)
\end{array}\right]-\sum_{n=1}^{2 m-1} \tilde{b}_{n j}(\theta) u(\tau-n)
$$

we have

$$
\left|\phi_{i}^{\prime} e_{j}(\tau)\right| \leq N p_{0} \max _{\tau-2 m+1 \leq s \leq \tau}\|\Delta(s)\|_{\infty} \gamma^{\tau-2 m}\|S\|_{\infty}+N(2 m-1) b^{*} \lambda_{i} \varepsilon^{1 / 2} \bar{C} p_{0} \gamma^{\tau-4 m+1}
$$

and

$$
\begin{aligned}
\left\|\epsilon_{i}(\tau)\right\|_{\infty} \leq & \lambda_{i} c^{*} \varepsilon 2 m N\left[p_{0} \max _{\tau-2 m+1 \leq s \leq \tau}\|\Delta(s)\|_{\infty} \gamma^{\tau-2 m}\|S\|_{\infty}\right. \\
& \left.+(2 m-1) b^{*} \lambda_{i} \varepsilon^{1 / 2} \bar{C} p_{0} \gamma^{\tau-4 m+1}\right] \\
\leq & 2 \lambda_{i} c^{*} m N\left(\|S\|_{\infty} / 2+1\right) p_{0} \gamma^{\tau-2 m} \varepsilon
\end{aligned}
$$


by (5.41c), if $\|\Delta(s)\|_{\infty} \leq 1 / 2$ for $\tau-2 m+1 \leq s \leq \tau$.

Recalling (5.44) we obtain

$$
\tilde{\delta}^{i}(t+1)=A_{i}^{t+1-2 m} \tilde{\delta}^{i}(2 m)-\sum_{\tau=0}^{t-2 m} A_{i}^{t-2 m-\tau} \epsilon_{i}(2 m+\tau) .
$$

By applying Lemma 5.4 and taking into account 5.46 , it yields that

$$
\leq\left\{\begin{array}{l}
\left|\tilde{\delta}_{i, 2 j-1}(t+1)\right|,\left|\tilde{\delta}_{i, 2 j}(t+1)\right| \\
j=1, \ldots, m-2 ; \\
M_{i j} \varepsilon^{j-(m-1)} \gamma^{t+1-2 m}\left[\left\|\tilde{\delta}^{i}(2 m)\right\|_{\infty}+4 \lambda_{i} c^{*} m N\left(\|S\|_{\infty}+2\right) p_{0}\right], \\
j=m-1, m,
\end{array}\right.
$$

due to $\varepsilon /\left(\gamma-\rho_{i}\right)<4$.

With 5.47) it is ready to estimate

$$
\phi_{i}^{\prime} u(t+1)=\sum_{j=1}^{2 m} k_{j} \phi_{i}^{\prime} L \delta_{j}(t+1)+\sum_{j=1}^{2 m} k_{j} \phi_{i}^{\prime} e_{j}(t+1) .
$$

For the first part, by (5.47) and $\left\|\tilde{\delta}^{i}(2 m)\right\|_{\infty} \leq\left\|T_{L}^{-1}\right\|_{\infty}\left\|\delta_{i}(2 m)\right\|_{\infty}$ we have

$$
\begin{aligned}
& \left|\sum_{j=1}^{2 m} k_{j} \phi_{i}^{\prime} L \delta_{j}(t+1)\right| \\
= & \lambda_{i}\left|\sum_{j=1}^{2 m} k_{j} \tilde{\delta}_{i j}(t+1)\right| \\
\leq & \lambda_{i} p_{0} \gamma^{t-2 m+1}\left(2 M_{i, m-1} \varepsilon^{1 / 2}+2 c^{*} \varepsilon \sum_{j \neq m-1} M_{i j}\right) . \\
& {\left[\left\|\tilde{\delta}^{i}(2 m)\right\|_{\infty}+4 \lambda_{i} c^{*} m N\left(\|S\|_{\infty}+2\right) p_{0}\right] } \\
\leq & 3 \lambda_{i} M_{i, m-1}\left[\left\|T_{L}^{-1}\right\|_{\infty}+4 \lambda_{i} c^{*} m N\left(\|S\|_{\infty}+2\right)\right] p_{0} \gamma^{t-2 m+1} \varepsilon^{1 / 2}
\end{aligned}
$$

if we note that $\left|c_{2 m-2}\right|,\left|c_{2 m-3}\right| \leq 1$. For the second part, as in the second order case, it is closely related with $\|\Delta(t+1)\|_{\infty}$ and similarly it can be inferred from 5.33 that $d(t+1)$ is only dependent on the past quantization errors $\Delta(\tau), t-2 m+1 \leq \tau \leq t$ and the past control inputs $u(\tau), t-2 m+1 \leq \tau \leq t-1$. Hence, under the induction assumption (5.45) the quantizer can be made unsaturated at time $t+1$ with finite bits, namely $\|\Delta(t+1)\|_{\infty} \leq 1 / 2$. In consequence we get the following estimate similar to 5.46 as

$$
\left|\sum_{j=1}^{2 m} k_{j} \phi_{i}^{\prime} L e_{j}(t+1)\right| \leq 2 \lambda_{i} c^{*} m N\left(\frac{1}{2}\|S\|_{\infty}+1\right) p_{0} \gamma^{t-2 m+1} \varepsilon .
$$

Combining (5.48) and (5.49), it is clear that $\left|\phi_{i}^{\prime} u(t+1)\right| \leq \Lambda_{i} \bar{C} p_{0} \gamma^{t-2 m+1} \varepsilon^{1 / 2}$, which establishes the 
induction. Furthermore, by (5.47) clearly the consensus can be achieved at a convergence rate of $O\left(\gamma^{t}\right)$.

3) Data rate. Below we are to discuss the number of quantization levels at each time step. The situation when $t \leq 2 m$ has been discussed. When $t>2 m$, we have

$$
\begin{aligned}
& \|d(t)\|_{\infty} \\
\leq & \left\|\frac{\cos \theta}{\gamma} \Delta(t-1)+\sum_{j=1}^{2 m} \frac{\sin \theta S(2, j)+S(3, j)}{\gamma^{2 m-j+1}} \Delta(t-1-2 m+j)\right\|_{\infty}+2 b^{*} \sum_{j=1}^{2 m-1} \frac{\|u(t-1-j)\|_{\infty}}{p(t-1)} \\
= & \left\|\sum_{j=1}^{2 m} \frac{1}{\gamma^{2 m-j+1}}[\cos \theta S(1, j)+\sin \theta S(2, j)+S(3, j)] \Delta(t-1-2 m+j)\right\|_{\infty} \\
& +2 b^{*} \sum_{j=1}^{2 m-1} \frac{\|u(t-1-j)\|_{\infty}}{p(t-1)} \\
\leq & \frac{1}{2 \gamma^{2 m}} \sum_{k=0}^{2 m-1}\left|\pi_{k}(\theta)\right|+2 b^{*}(2 m-1)(N-1) \Lambda \bar{C} \gamma^{1-4 m} \varepsilon^{1 / 2}
\end{aligned}
$$

by noticing $S(1, \cdot)=[0, \ldots, 0,1]$ and $u(t)=\sum_{i=1}^{N} \phi_{i} \phi_{i}^{\prime} u(t), \Lambda_{1}=0$. By taking into account 5.41b and (5.41c) it can be seen that $\|d(t)\|_{\infty}$ is bounded by $2^{m-1}(1+|\cos \theta|)^{m}$ and the proof is completed by remembering 5.42 .

Remark 5.10. Noticing that $\prod_{1 \leq k \leq m-2, k \neq n}|k-n|=(n-1) !(m-2-n)$ ! attains the minimum at $n=\left\lfloor\frac{m-2}{2}\right\rfloor$ and multiplying by a positive $\lambda_{i}$ on both sides does not change the direction of an inequality, 5.41a can be substituted by the following stronger one, which is easier to check:

$$
5 c^{*}\left(\sum_{j=1}^{m-2} \sum_{n=1}^{m-2} \frac{n^{j-1}}{\left(\left\lfloor\frac{m}{2}\right\rfloor-2\right) !\left(m-1-\left\lfloor\frac{m}{2}\right\rfloor\right) !}+1+\lambda_{N}\right) \varepsilon^{1 / 2} \leq 3 \sqrt{\frac{\lambda_{2}}{2}} .
$$

Remark 5.11. From the proof it is readily seen that we can still use the same number of bits to achieve the quantized consensus once the Laplacian of the directed topology satisfies that $0<\lambda_{2} \leq \cdots \leq \lambda_{N}$. However, unlike the case of the 2nd-order oscillator, it does not hold for general topology, when the Laplacian contains complex eigenvalues, or real Jordan blocks of multiple dimensions. For one reason, note that Lemma 5.3 does not hold for a complex $\lambda_{i}$. For another one, note the disparity in the order of $\varepsilon$ between the disturbance term and the weighted sum of disagreement entries, i.e. $\left\|\epsilon_{i}(t)\right\|_{\infty}=O(\varepsilon) p_{0} \gamma^{t}$ and $\left\|K \tilde{\delta}^{i}(t)\right\|_{\infty}=O\left(\varepsilon^{1 / 2}\right) p_{0} \gamma^{t}$. Therefore, if we assume $m=2$ and the Jordan block corresponding to $\lambda_{2}>0$ is two-dimensional as in (5.17), then it follows from $\left\|K \tilde{\delta}^{3}(t)\right\|_{\infty}=O\left(\varepsilon^{1 / 2}\right) p_{0} \gamma^{t}$ that $\left\|\tilde{\delta}^{2}(t)\right\|_{\infty}=$ $O\left(\varepsilon^{-1}\right) p_{0} \gamma^{t}$ and $\|u(t)\|_{\infty}=O(1) p_{0} \gamma^{t}$, suggesting that the input term can no longer be neglected in the estimation errors, nor in the quantization input $d(t)$. Also see Remark 4.14 for a similar discussion.

Remark 5.12. At the first glance it may seem doubtful that the data rate is dependent on $|\cos \theta| ;$ but a little further inspection is enough to clarify. Similar to the situation of the $n$-th order integrator system investigated in Chapter 4 , the control input does not consume any bit in exchanging the estimates of the states when $\varepsilon$ is sufficiently small. In other words, we only need to focus on how many bits it needs to estimate the output of an individual open loop system. Take the second-order case as an 

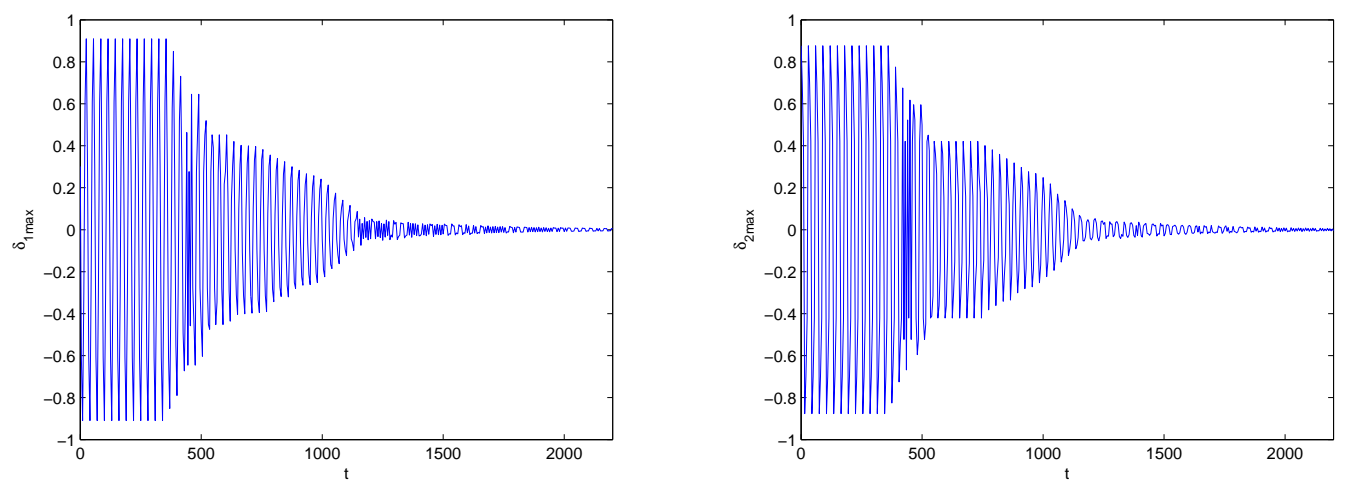

Figure 5.1: Convergence of maximum disagreements $\delta_{\text {jmax }}(t): \mathrm{m}=1$.

example. Noticing that $y_{i}(t)=\cos \theta x_{i 1}(t-1)+\sin \theta x_{i 2}(t-1)=2 \cos \theta x_{i 1}(t-1)-x_{i 1}(t-2)$, we can estimate $y_{i}(t)$ based on $\hat{x}_{i 1}(t-1)$ and $\hat{x}_{i 1}(t-2)$ with an error bound no larger than $\frac{1}{2}(2|\cos \theta|+1)+\frac{1}{2}$. Generally speaking, when $|\cos \theta| \approx 0$ or equivalently $|\sin \theta| \approx 1, x_{i, 2 j-1}(t)$ and $x_{i, 2 j}(t)$ are tightly coupled, and it needs only $m$ bits of information exchange to achieve the consensus; in the case of $|\cos \theta| \approx 1$, after rearranging of states $A$ can be approximated by $I_{2} \otimes J_{1, m}$, and $2 m$ bits are sufficient. In summary, for a $2 m$-th order system studied in this chapter, a data rate between $m$ and $2 m$ bits is sufficient for achieving the consensus asymptotically, and the exact rate is dependent on the oscillator frequency. This result is consistent with the conclusion for $n$-th order integrator systems studied in the last chapter.

\subsection{Numerical Examples}

In this section we consider two examples respectively corresponding to $m=1$ and $m=2$.

For the first case, consider a network of 5 harmonic oscillators connected as a unidirectional ring, with 0 - 1 edge weights. Given $\theta=\pi / 3$, it only needs a single bit of information exchange to achieve the consensus. With $L=\left[\begin{array}{ccccc}1 & -1 & & & \\ & 1 & -1 & & \\ & & 1 & -1 & \\ & & & 1 & -1 \\ & & & & 1\end{array}\right]$, it can be found that $\operatorname{Re} \lambda_{2}=0.6910$, and we select $c_{1}=-1.2533$ and $c_{2}=0.7236$ by letting $h=\operatorname{Re} \lambda_{2}$ in Remark 5.3 . For the simulation we assume that $\left|x_{i j}(0)\right| \leq 2$, and take $\varepsilon=0.01, p_{0}=10, \gamma=0.9975$ to satisfy the conditions in Theorem 5.1. From Fig. 5.1 which depicts the trajectory of maximum disagreements $\delta_{j \max }(t)=\left\{\delta_{n j}(t): n=\arg \max _{i}\left|\delta_{i j}(t)\right|\right\}$ for $j=1,2$, we can see that the consensus is achieved asymptotically. 

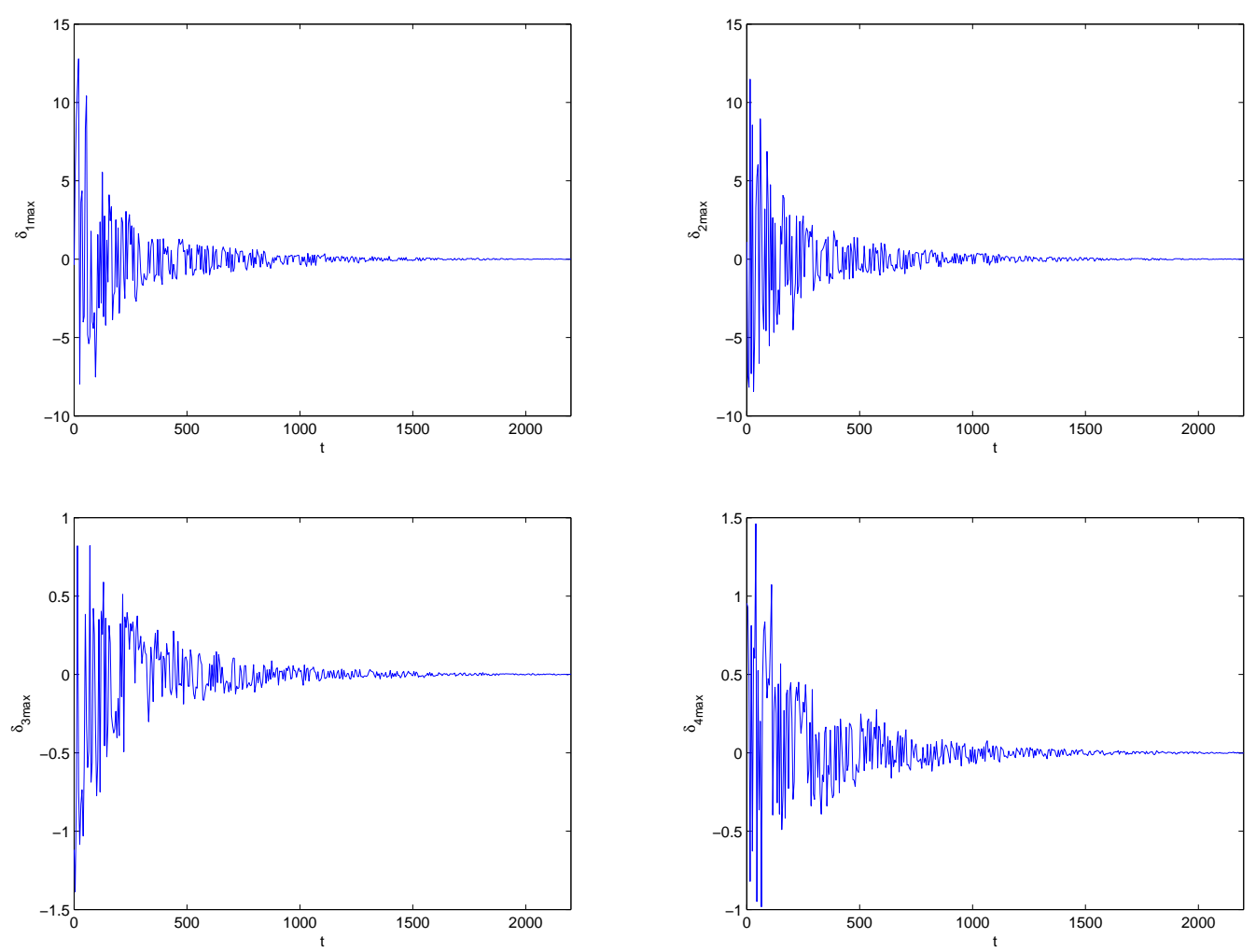

Figure 5.2: Convergence of maximum disagreements $\delta_{\text {jmax }}(t): \mathrm{m}=2$.

For the case of $m=2$, consider a 5-node network with 4-th order dynamics described by (5.1), and assume that they are connected as an undirected ring with $0-1$ edge weights. Given $\theta=$ $\pi / 3$, it needs 3 bits of information exchange to achieve the consensus, and we can compute $S_{2}=$ $\left[\begin{array}{cccc}-4 /(3 \sqrt{3}) & 2 / \sqrt{3} & -4 /(3 \sqrt{3}) & 5 /(3 \sqrt{3}) \\ -1 / 3 & 1 & -1 & 2 / 3 \\ -1 / \sqrt{3} & 1 / \sqrt{3} & -1 / \sqrt{3} & 0\end{array}\right]$ to construct the encoder and decoder respectively as 5.29 - With $L=\left[\begin{array}{ccccc}2 & -1 & & & -1 \\ -1 & 2 & -1 & & \\ & -1 & 2 & -1 & \\ & & -1 & 2 & -1 \\ -1 & & & -1 & 2\end{array}\right]$, it can be found that $\lambda_{2}=1.3820$, and we select $c_{j}$ 's according to Remark 5.8 by letting $h=\lambda_{2}$. For the simulation we assume that $\left|x_{i j}(0)\right| \leq 4$, and take $\varepsilon=0.01, p_{0}=10, \gamma=0.9975$ to satisfy the conditions in Theorem 5.2. From Fig. 5.2 which depicts the trajectory of maximum disagreements $\delta_{\text {jmax }}(t)=\left\{\delta_{n j}(t): n=\arg \max _{i}\left|\delta_{i j}(t)\right|\right\}$ for $j=1,2,3,4$, we can see that the consensus is achieved asymptotically. 


\subsection{Conclusion}

In this chapter, we explored the data rate problem for quantized consensus of MASs with another kind of critical high-order dynamics. Different from the high-order integrator case, the system matrix of each agent is described by a $2 m$-th order real Jordan form consisting of $m$ pairs of conjugate poles on the unit circle. Besides, each system has single input, and only the first state variable can be measured. To estimate the other state variables, a novel encoding-decoding scheme was devised based on the observability matrix. Perturbation techniques were employed in the consensus analysis and the data rate analysis, and combinatorial techniques were used to derive an explicit expression of the data rate. The second-order case of $m=1$ and higher-order cases of $m \geq 2$ were investigated separately. For the second-order case, we showed that at most 2 bits of information exchange suffice to achieve the consensus at an exponential rate, if the communication topology has a spanning tree. For higher-order cases, consensus can be achieved with at most $2 m$ bits, provided that the undirected communication topology is connected. The exact number of bits for achieving consensus in both cases is an integer which increases from $m$ to $2 m$ when $|\cos \theta|$ increases from 0 to 1 . It is more important to note that depending on the frequency of oscillators, the consensus can be achieved by less than $2 m$ or even only $m$ bits of information exchange for $2 m$-th order oscillator systems, which is quite different from the result of integrator systems and reveals an interesting connection between the data rate and the system dynamics.

\subsection{Proof of Main Lemmas}

Proof of Lemma 5.3. Here we mainly deal with the case of $m \geq 3$, since the proof can be easily adapted for $m=2$ and the modification will be pointed out accordingly. The characteristic equation of $A_{i}$ can be computed as

$$
\chi_{i}(\mu)=\operatorname{det}\left[(\mu I-Q)^{m}+\lambda_{i} K_{m}(\mu I-Q)^{m-1}+\cdots+\lambda_{i} K_{2}(\mu I-Q)+\lambda_{i} K_{1}\right]
$$




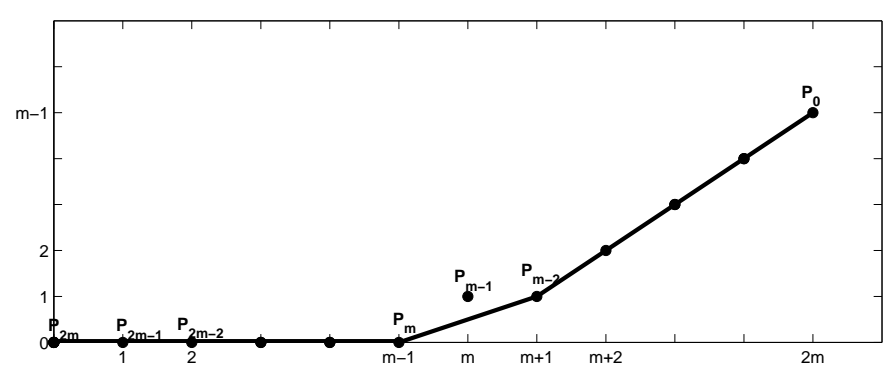

Figure 5.3: Newton diagram.

where $Q=\left[\begin{array}{cc}\cos \theta & \sin \theta \\ -\sin \theta & \cos \theta\end{array}\right]$ and $K_{j}=\left[\begin{array}{cc}0 & 0 \\ k_{2 j-1} & k_{2 j}\end{array}\right]$ for $j=1, \ldots, m$. By employing (5.10) in the proof of Lemma 5.1, we rewrite $\chi_{i}(\mu)$ as

$$
\begin{aligned}
\chi_{i}(\mu)= & \left(\mu-e^{\jmath \theta}\right)^{m}\left(\mu-e^{-\jmath \theta}\right)^{m} \\
& +\frac{1}{2}\left[\left(\mu-e^{\jmath \theta}\right)^{m}+\left(\mu-e^{-\jmath \theta}\right)^{m}\right] \frac{\lambda_{i}}{2} . \\
& {\left[\sum_{j=2}^{m}\left(-k_{2 j-1} \jmath+k_{2 j}\right)\left(\mu-e^{\jmath \theta}\right)^{j-1}+2 k_{2}+\sum_{j=2}^{m}\left(k_{2 j-1} \jmath+k_{2 j}\right)\left(\mu-e^{-\jmath \theta}\right)^{j-1}\right] } \\
& +\frac{\jmath}{2}\left[\left(\mu-e^{\jmath \theta}\right)^{m}-\left(\mu-e^{-\jmath \theta}\right)^{m}\right] \frac{\lambda_{i}}{2} . \\
& {\left[\sum_{j=2}^{m}\left(k_{2 j-1}+k_{2 j} \jmath\right)\left(\mu-e^{\jmath \theta}\right)^{j-1}+2 k_{1}+\sum_{j=2}^{m}\left(k_{2 j-1}-k_{2 j} \jmath\right)\left(\mu-e^{-\jmath \theta}\right)^{j-1}\right] } \\
= & \left(\mu-e^{\jmath \theta}\right)^{m}\left[\left(\mu-e^{-\jmath \theta}\right)^{m}+\frac{\lambda_{i}}{2}\left(k_{2}+k_{1} \jmath\right)\right] \\
& +\frac{\lambda_{i}}{2}\left(\mu-e^{-\jmath \theta}\right)^{m} \sum_{j=1}^{m}\left(\mu-e^{\jmath \theta}\right)^{j-1}\left(-k_{2 j-1} \jmath+k_{2 j}\right) .
\end{aligned}
$$

With $A_{i}$ being real, its complex eigenvalues will always occur in complex conjugate pairs. Therefore, we only need to focus on the perturbed roots around $e^{\jmath \theta}$, which are denoted by $\mu=e^{\jmath \theta}+\Delta \mu$. Noticing that $\mu-e^{-\jmath \theta}=\mu-e^{\jmath \theta}+2 \sin \theta$, we substitute $\mu=e^{\jmath \theta}+\Delta \mu$ into (5.51) and obtain

$$
\chi_{i}\left(e^{\jmath \theta}+\Delta \mu\right)=\sum_{n=1}^{m} a_{i n}(\varepsilon)(\Delta \mu)^{m-n}+\sum_{n=0}^{m}\left[C(m, m-n)(2 \sin \theta \jmath)^{m-n}+O(\varepsilon)\right](\Delta \mu)^{m+n}
$$

with the selection of $k_{2 j-1}$ and $k_{j}$ in (5.36), where

$$
a_{i n}(\varepsilon)=\left\{\begin{array}{l}
(2 \sin \theta \jmath)^{m-1}\left[2 \sin \theta \jmath\left(c_{2 m}-c_{2 m-1} \jmath\right)+m\left(c_{2 m-2}-c_{2 m-3} \jmath\right)\right] \varepsilon+o(\varepsilon), n=1 \\
(2 \sin \theta \jmath)^{m}\left(c_{2(m-n+1)}-c_{2(m-n)+1}\right) \varepsilon^{n-1}+o\left(\varepsilon^{n-1}\right), n=2, \ldots, m .
\end{array}\right.
$$

Now the Newton diagram 108 can be depicted as in Fig. 5.3 . by first plotting points $P_{2 m-j}\left(j, \alpha_{2 m-j}\right)$, 
$j=0, \ldots, 2 m$ and then connecting the segments on the lower boundary of the convex hull of the above points, where $\alpha_{2 m-j}$ is the leading exponent of $\varepsilon$ in the coefficient of $(\Delta \mu)^{2 m-j}$. The slopes of the two non-horizontal segments are $1 / 2,1$ respectively, implying that $\Delta \mu$ has the following two forms of expansions:

$$
\begin{array}{r}
\Delta \mu=\mu_{1} \varepsilon^{\frac{1}{2}}+\mu_{2} \varepsilon^{\beta}+o\left(\varepsilon^{\beta}\right), \mu_{1} \neq 0 \\
\Delta \mu=v_{1} \varepsilon+o(\varepsilon), \vartheta_{1} \neq 0 .
\end{array}
$$

Substituting 5.53a into 5.52 and finding the coefficients of the term $\varepsilon^{m / 2}$, it yields that $\mu_{1}^{m}(2 \jmath \sin \theta)^{m}+\mu_{1}^{m-2} \frac{\lambda_{i}}{2}(2 \jmath \sin \theta)^{m}\left(c_{2 m-2}-c_{2 m-3} \jmath\right)=0$, and thus

$$
\mu_{1}= \pm \sqrt{\frac{\lambda_{i}}{2} \sqrt{c_{2 m-3}^{2}+c_{2 m-2}^{2}}} e^{\jmath \frac{\alpha}{2}}
$$

where $\alpha=\arg \left(c_{2 m-3} \jmath-c_{2 m-2}\right)$. Moreover, to determine $\mu_{2}$ and $\beta$, we substitute 5.53a into 5.52 again and find the lowest order term as

$$
\begin{aligned}
& m \mu_{1}^{m-1} \mu_{2}(2 \jmath \sin \theta)^{m} \varepsilon^{(m-1) / 2} \varepsilon^{\beta} \\
+ & \frac{\lambda_{i}}{2} \mu_{1}^{m-1}(2 \jmath \sin \theta)^{m}\left(c_{2 m}-c_{2 m-1} \jmath\right) \varepsilon^{(m+1) / 2} \\
+ & \frac{\lambda_{i}}{2}(m-2) \mu_{1}^{m-3} \mu_{2}(2 \jmath \sin \theta)^{m}\left(c_{2 m-2}-c_{2 m-3} \jmath\right) \varepsilon^{(m-1) / 2} \varepsilon^{\beta} \\
+ & \frac{\lambda_{i}}{2} \mu_{1}^{m-3}(2 \jmath \sin \theta)^{m}\left(c_{2 m-4}-c_{2 m-5} \jmath\right) \varepsilon^{(m+1) / 2}=0,
\end{aligned}
$$

which implies $\beta=1$ and $\mu_{2}=\frac{\lambda_{i}}{4}\left(c_{2 m-1} \jmath-c_{2 m}\right)+\frac{\left(c_{2 m-5 \jmath}-c_{2 m-4}\right)}{2\left(c_{2 m-3}-c_{2 m-2}\right)}$. In the form of 5.53a), the module of $\mu$ is determined as

$$
\begin{aligned}
|\mu|^{2} & =\mu \bar{\mu} \\
& =\left(e^{\jmath \theta}+\mu_{1} \varepsilon^{\frac{1}{2}}+\mu_{2} \varepsilon^{\beta}+o(\varepsilon)\right)\left(e^{-\jmath \theta}+\bar{\mu}_{1} \varepsilon^{\frac{1}{2}}+\bar{\mu}_{2} \varepsilon^{\beta}+o(\varepsilon)\right) \\
& =1+\left(\mu_{1} e^{-\jmath \theta}+\bar{\mu}_{1} e^{\jmath \theta}\right) \varepsilon^{\frac{1}{2}}+\left(\mu_{1} \bar{\mu}_{1}+\mu_{2} e^{-\jmath \theta}+\bar{\mu}_{2} e^{\jmath \theta}\right) \varepsilon+o(\varepsilon) \\
& =1+2 \operatorname{Re}\left(\mu_{1} e^{-\jmath \theta}\right) \varepsilon^{\frac{1}{2}}+\left(\left|\mu_{1}\right|^{2}+2 \operatorname{Re}\left(\mu_{2} e^{-\jmath \theta}\right)\right) \varepsilon+o(\varepsilon),
\end{aligned}
$$

with $\operatorname{Re}\left(\mu_{1} e^{-\jmath \theta}\right)= \pm \sqrt{\frac{\lambda_{i}}{2} \sqrt{c_{2 m-3}^{2}+c_{2 m-2}^{2}}} \cos \left(\frac{\alpha}{2}-\theta\right)$. In order that $|\mu|<1$ with sufficiently small $\varepsilon$, we must have $\frac{\alpha}{2}-\theta=\frac{\pi}{2}$, and hence it suffices to let $\left|\mu_{1}\right|^{2}+2 \operatorname{Re}\left(\mu_{2} e^{-\jmath \theta}\right)<0$. Combining these arguments gives rise to a sufficient condition as

$$
\begin{gathered}
-c_{2 m-3} / c_{2 m-2}=\tan (2 \theta+\pi), c_{2 m-3}^{2}+c_{2 m-2}^{2} \neq 0 \\
\frac{\lambda_{i}}{2} \sqrt{c_{2 m-3}^{2}+c_{2 m-2}^{2}}+\frac{\lambda_{i}}{2}\left(c_{2 m-1} \sin \theta-c_{2 m} \cos \theta\right)+\operatorname{Re}\left(\frac{c_{2 m-5} \jmath-c_{2 m-4}}{c_{2 m-3} \jmath-c_{2 m-2}} e^{-\jmath \theta}\right)<0
\end{gathered}
$$

With $c_{2 m-3}=-\sin 2 \theta$ and $c_{2 m-2}=\cos 2 \theta$ satisfying $5.57 \mathrm{a}$, 5.57b is equivalent to $\lambda_{i} R_{m}+H<0$. 
When $m=2, \mu$ only takes the form of (5.53a) and $\mu_{2}=\frac{\lambda_{i}}{4}\left(c_{2 m-1} \jmath-c_{2 m}\right)$, leading to the sufficient condition $R_{2}<0$ for $|\mu|<1$.

On the other hand, substituting 5.53b into 5.51) and finding the coefficients of the term $\varepsilon^{m-1}$, we obtain the equation 5.38 . Similarly, the module of $\mu$ with the form $5.53 \mathrm{~b}$ is determined by

$$
|\mu|^{2}=1+2 \operatorname{Re}\left(\vartheta_{1} e^{-\jmath \theta}\right) \varepsilon+o(\varepsilon)
$$

and it suffices to let $\operatorname{Re}\left(\vartheta_{1} e^{-\jmath \theta}\right)$ be negative so that $|\mu|<1$ with sufficiently small $\varepsilon$. For the given $c_{2 m-3}$ and $c_{2 m-2}$, the roots of 5.38 can be assigned arbitrarily such that $\operatorname{Re}\left(\vartheta_{1} e^{-\jmath \theta}\right)<0$ with $m-2$ distinct $\vartheta_{1}$; once $c_{2 m-4}$ and $c_{2 m-5}$ have been determined, $5.57 \mathrm{~b}$ can always be satisfied by properly choosing $c_{2 m}$ and $c_{2 m-1}$ since $\frac{\lambda_{i}}{2}\left(c_{2 m-1} \sin \theta-c_{2 m} \cos \theta\right)$ can be assigned to any number. In summary, the proof is completed.

Proof of Lemma 5.4. As with the proof of Lemma 5.3, we only focus on the case of $m \geq 3$ which essentially includes the case of $m=2$. For $A_{i}$, we are to find the following Jordan decomposition:

$$
A_{i}=A+\sum_{j=1}^{m-1} A_{i j} \varepsilon^{j}=R_{i} \tilde{A}_{i} R_{i}^{-1}
$$

where $\tilde{A}_{i}$ is a diagonal matrix consisting of $2 m$ different eigenvalues determined in Lemma 5.3 . To find an appropriate $R_{i}$ and the corresponding $R_{i}^{-1}$, we first determine the Jordan basis of the unperturbed matrix $A$. The Jordan chain corresponding to the eigenvalue $\mu_{0}=e^{\jmath \theta}$ is given by

$$
u_{m-1} \stackrel{A-\mu_{0} I}{\longrightarrow} u_{m-2} \stackrel{A-\mu_{0} I}{\longrightarrow} \ldots \stackrel{A-\mu_{0} I}{\longrightarrow} u_{1} \stackrel{A-\mu_{0} I}{\longrightarrow} u_{0}
$$

where $u_{j}=e_{2 j+1}+\jmath e_{2 j+2}, j=0, \ldots, m-1$ and $e_{n} \in \mathbb{R}^{2 m}$ denotes the vector with a 1 in the $n$-th coordinate and 0's elsewhere. Similarly, the Jordan chain corresponding to the eigenvalue $\bar{\mu}_{0}=e^{-\jmath \theta}$ is given by

$$
\bar{u}_{m-1} \stackrel{A-\bar{\mu}_{0} I}{\longrightarrow} \bar{u}_{m-2} \stackrel{A-\bar{\mu}_{0} I}{\longrightarrow} \ldots \stackrel{A-\bar{\mu}_{0} I}{\longrightarrow} \bar{u}_{1} \stackrel{A-\bar{\mu}_{0} I}{\longrightarrow} \bar{u}_{0}
$$

Hence the two Jordan chains of $A$ can be rearranged as $R_{0}=\left[\begin{array}{lllll}u_{0} & \bar{u}_{0} & \ldots & u_{m-1} & \bar{u}_{m-1}\end{array}\right]=I_{m} \otimes P$ with $P=\left[\begin{array}{cc}1 & 1 \\ \jmath & -\jmath\end{array}\right]$. With $A_{i}$ being real, once we obtain the eigenvectors corresponding to the $m$ different perturbed eigenvalues around $\mu_{0}$, the other eigenvectors can be obtained by taking conjugates. Hence we only need to find the eigenvectors corresponding to the $m$ different perturbed eigenvalues around $\mu_{0}$.

The eigenvectors corresponding to the $m$ perturbed eigenvalues around $\mu_{0}$ have the following 
form of Puiseux series 109:

$$
\begin{array}{lll}
\mu_{i n}=\mu_{0}+\sum_{k=1}^{\infty} \mu_{i n k} \varepsilon^{k / 2}, & u_{i n}=u_{i n 0}+\sum_{k=1}^{\infty} u_{i n k} \varepsilon^{k / 2}, & n=1,2 ; \\
\mu_{i n}=\mu_{0}+\sum_{k=1}^{\infty} \vartheta_{i n k} \varepsilon^{k}, & u_{i n}=u_{i n 0}+\sum_{k=1}^{\infty} u_{i n k} \varepsilon^{k}, & n=3, \ldots, m, m
\end{array}
$$

where $\mu_{i 11}=\sqrt{\frac{\lambda_{i}}{2} \sqrt{c_{2 m-3}^{2}+c_{2 m-2}^{2}}} e^{\jmath \frac{\alpha}{2}}, \mu_{i 21}=-\mu_{i 11}$ and $\vartheta_{i n 1}=\vartheta_{n 1}, n=3, \ldots, m$ have been defined in Lemma 5.3. Substituting $\mu_{i n}, u_{i n}$ into the equation $A_{i} u_{i n}=\mu_{i n} u_{i n}$ respectively, and collecting coefficients of equal powers of $\varepsilon$; moreover, noticing the fact that $A_{i j} u_{k}=0, j=1, \ldots, m-2 ; k=$ $0, \ldots, m-2-j$, where $A_{i j}$ has been defined in 5.59 and imposing the normalization condition as $v_{m-1}^{\prime} u_{i n}=1$, where $v_{m-1}=\frac{1}{2}[1,-\jmath, 0, \ldots, 0]^{\prime}$ is the left associated eigenvector of $A$ with respect to the eigenvalue $\mu_{0}$ such that $v_{m-1}^{\prime} u_{0}=1, v_{m-1}^{\prime} u_{1}=\cdots=v_{m-1}^{\prime} u_{m-1}=0$, the $m$ eigenvectors can be obtained as:

$$
\begin{array}{ll}
u_{i n}=u_{0}+\sum_{k=1}^{m-1} \varepsilon^{k / 2}\left(\mu_{i 1 n}^{k} u_{k}+u_{i k n}^{\prime}\right)+o\left(\varepsilon^{m / 2}\right), & n=1,2 ; \\
u_{i n}=u_{0}+\sum_{k=1}^{m-1} \varepsilon^{k}\left(\vartheta_{1 n}^{k} u_{k}+u_{i k n}^{\prime}\right)+o\left(\varepsilon^{m}\right), & n=3, \ldots, m,
\end{array}
$$

where $u_{i 1 n}^{\prime}=0$ and $u_{i k n}^{\prime} \in \operatorname{span}\left\{u_{1}, \ldots, u_{k-1}\right\}, k=2, \ldots, m-1$ for $n=1, \ldots, m$.

Letting $R_{i}=\left[u_{i 1}, \bar{u}_{i 1}, \ldots, u_{i m}, \bar{u}_{i m}\right]$, we are to investigate the magnitude of each entry in $R_{i}^{-1}$ by adjoint method. To this end, we need to find the order of $\operatorname{det} R_{i}$ and the corresponding cofactor, both of which can be expressed as Puiseux series. The following facts should be considered before the calculation:

1). Determinant is a multi-linear function of column vectors, and it vanishes when two or more columns coincide.

2 ). There exist two types of series in the columns of $R_{i}$, and we categorize $u_{i 1}, u_{i 2}$ and their conjugates as type I, the others as type II.

With these two facts, we can see that the lowest degree can be obtained by taking out terms with $\varepsilon^{(m-2) / 2} u_{m-2}$ and $\varepsilon^{(m-1) / 2} u_{m-1}$ respectively from $u_{i 1}$ and $u_{i 1}$, terms with $u_{0}, \varepsilon u_{1}, \ldots, \varepsilon^{m-3} u_{m-3}$ respectively from $u_{i 3}, \ldots, u_{i m}$, as well as the corresponding conjugates from $\bar{u}_{i 1}, \ldots, \bar{u}_{i m}$, and calculated by $2\left(0+1+\cdots+m-3+\frac{m-2}{2}+\frac{m-1}{2}\right)=m^{2}-3 m+3$. Moreover,

$$
\begin{aligned}
\left|\operatorname{det} R_{i}\right| & =\left|\mu_{i 11}^{m-1} \mu_{i 21}^{m-2}-\mu_{i 11}^{m-2} \mu_{i 21}^{m-1}\right|^{2}\left|\operatorname{det} V_{0}\right|^{2}\left|\operatorname{det} R_{0}\right| \varepsilon^{m^{2}-3 m+3}(1+o(1)) \\
& =\left|\mu_{i 11}^{m-2} \mu_{i 21}^{m-2}\left(\mu_{i 11}-\mu_{i 21}\right)\right|^{2}\left|\operatorname{det} V_{0}\right|^{2} 2^{m} \varepsilon^{m^{2}-3 m+3}(1+o(1)),
\end{aligned}
$$


where $V_{0}=V\left(\vartheta_{31}, \ldots, \vartheta_{m 1}\right)=\left[\begin{array}{cccc}1 & \vartheta_{31} & \ldots & \vartheta_{31}^{m-3} \\ 1 & \vartheta_{41} & \ldots & \vartheta_{41}^{m-3} \\ \vdots & \vdots & \ddots & \vdots \\ 1 & \vartheta_{m 1} & \ldots & \vartheta_{m 1}^{m-3}\end{array}\right]$ is a Vandermonde matrix of order $m-2$.

On the other hand, we need to determinate the order of the cofactor $C_{s, t}^{(i)}$ with respect to the $(s, t)$ entry, and we illustrate it by calculating $C_{1,1}^{(i)}$ with $m=3$. After deleting the first column $u_{i 1}$, we delete the first row and use the same notations $u_{1}, u_{2}$ and $e_{2}, \ldots, e_{6}$. Now $R_{i}$ has been reduced to a square matrix $R_{1,1}^{(i)}$ consisting of the following 5 columns:

$$
\begin{aligned}
& a_{1}=-j e_{2}+\varepsilon^{1 / 2} \bar{\mu}_{i 11} \bar{u}_{1}+\varepsilon \bar{\mu}_{i 11}^{2} \bar{u}_{2}+O(\varepsilon) \bar{u}_{1}+O\left(\varepsilon^{2}\right), \\
& a_{2}=j e_{2}+\varepsilon^{1 / 2} \mu_{i 21} u_{1}+\varepsilon \mu_{i 21}^{2} u_{2}+O(\varepsilon) u_{1}+O\left(\varepsilon^{2}\right), \\
& a_{3}=\bar{a}_{2}, \\
& a_{4}=j e_{2}+\varepsilon \vartheta_{31} u_{1}+\varepsilon^{2} \vartheta_{31}^{2} u_{2}+O\left(\varepsilon^{2}\right) u_{1}+O\left(\varepsilon^{2}\right), \\
& a_{5}=\bar{a}_{4} .
\end{aligned}
$$

Consequently the order of $C_{1,1}^{(i)}$ is found in such a way: take out terms with $\varepsilon^{1 / 2} \bar{u}_{1}, \varepsilon \bar{u}_{2}$ respectively from $a_{1}, a_{3}$, terms with $e_{2}$ from $a_{5}$, terms with $\varepsilon u_{2}$ from $a_{2}$, terms with $\varepsilon u_{1}$ from $a_{4}$. Now that $a_{1}, a_{3}, a_{5}$ jointly contribute the same degree of $\frac{1}{2}\left(m^{2}-3 m+3\right)$ as $\bar{u}_{i 1}, \bar{u}_{i 2}, \bar{u}_{i 3}$, we are left to choose terms with $u_{1}$ and $u_{2}$ respectively from $a_{2}$ and $a_{4}$. The above can be conducted similarly for calculating the order of $C_{1,1}^{(i)}$ when $m>3$, and actually for every cofactor. Moreover, by the symmetry of conjugates, $C_{2 k-1,2 n-1}^{(i)}, C_{2 k-1,2 n}^{(i)}, C_{2 k, 2 n-1}^{(i)}, C_{2 k, 2 n}^{(i)}$ have an identical order. So we only focus on $C_{2 k-1,2 n-1}^{(i)}$ below. Reminded by the case of $C_{1,1}^{(i)}$ when $m=3$, we suffice to choose linearly independent terms with a lowest sum of degrees from the modified columns $u_{i j}$ for $j \neq n$, where $u_{k-1}$ has been subtracted from each column. Recall that in finding the order of $\left|\operatorname{det} R_{i}\right|$, terms with $\varepsilon u_{0}, \ldots, \varepsilon^{m-3} u_{m-3}$ from type II columns are first selected, and then terms with $\varepsilon^{(m-2) / 2} u_{m-2}, \varepsilon^{(m-1) / 2} u_{m-1}$ from type I columns. Such a method still applies in finding the order of cofactors, and we conclude that $C_{2 k-1,2 n-1}^{(i)}$ has the lowest order for fixed $n$ if and only if $k=m$. In other words, for any row in $\operatorname{adj} R_{i}$, the entries at the $2 m-1$-th and $2 m$-th column exclusively have the lowest order when compared with other entries in the same row. To be detailed,

$$
=\left\{\begin{array}{l}
\left|C_{2 m-1,2 n-1}^{(i)}\right|,\left|C_{2 m-1,2 n}^{(i)}\right|,\left|C_{2 m, 2 n-1}^{(i)}\right|,\left|C_{2 m, 2 n}^{(i)}\right| \\
2^{m-1}\left|\operatorname{det} V_{0}\right|^{2}\left|\mu_{i 11}^{m-2} \mu_{i 21}^{m-2}\left(\mu_{i 11}-\mu_{i 21}\right)\right|\left|\mu_{i n 1}^{m-2}\right| \\
\cdot \varepsilon^{m^{2}-3 m+3-(m-1) / 2}[1+o(1)], n=1,2 ; \\
2^{m-1}\left|\operatorname{det} V_{0}\right|\left|\operatorname{det} V_{n} \| \mu_{i 11}^{m-2} \mu_{i 21}^{m-2}\left(\mu_{i 11}-\mu_{i 21}\right)\right| \\
\cdot\left|\mu_{i 11}^{m-3} \mu_{i 21}^{m-3}\left(\mu_{i 11}-\mu_{i 21}\right)\right| \varepsilon^{m^{2}-3 m+3-(m-2)}[1+o(1)], n=3, \ldots, m,
\end{array}\right.
$$


where $V_{n}=V\left(\vartheta_{31}, \ldots, \vartheta_{n-1,1}, \vartheta_{n 1}, \ldots, \vartheta_{m 1}\right)$ is a Vandermonde matrix of order $m-3$. Together with 5.60 it yields that by $\mu_{i 11}=-\mu_{i 21}$

$$
\begin{aligned}
& \left|R_{i}^{-1}(2 n-1,2 m-1)\right|,\left|R_{i}^{-1}(2 n, 2 m-1)\right|,\left|R_{i}^{-1}(2 n-1,2 m)\right|,\left|R_{i}^{-1}(2 n, 2 m)\right| \\
= & \left\{\begin{array}{l}
\frac{1}{4\left|\mu_{i 11}\right|^{m-1}} \varepsilon^{-(m-1) / 2}(1+o(1)), n=1,2 ; \\
\frac{1}{2\left|\mu_{i 11}\right|^{2}} \frac{\left|\operatorname{det} V_{n}\right|}{\left|\operatorname{det} V_{0}\right|} \varepsilon^{-(m-1)}(1+o(1)), n=3, \ldots, m ;
\end{array}\right. \\
= & \left\{\begin{array}{l}
o\left(\varepsilon^{-(m-1) / 2}\right), n=1,2 ; \quad \text { for } 1 \leq k \leq 2 m . \\
o\left(\varepsilon^{-(m-1)}\right), n=3, \ldots, m ;
\end{array}\right.
\end{aligned}
$$

In the meanwhile, the following holds for $1 \leq j \leq m$ :

$$
\begin{aligned}
& \quad\left|R_{i}(2 j-1,2 n-1)\right|,\left|R_{i}(2 j-1,2 n)\right|,\left|R_{i}(2 j, 2 n-1)\right|,\left|R_{i}(2 j, 2 n)\right| \\
& =\left\{\begin{array}{l}
\left|\mu_{i n 1}^{j-1}\right| \varepsilon^{(j-1) / 2}+O\left(\varepsilon^{j / 2}\right), n=1,2 ; \\
\left|\vartheta_{n 1}^{j-1}\right| \varepsilon^{j-1}+O\left(\varepsilon^{j}\right), n=3, \ldots, m ;
\end{array}\right.
\end{aligned}
$$

Combining (5.59), 5.62 and 5.63) we can obtain

$$
\begin{aligned}
& \left|\xi_{s, 2 j-1}\right| \\
\leq & \rho_{i}^{s}\|\xi\|_{\infty}\left[\sum_{n=1}^{m}\left|R_{i}(2 j-1,2 n-1)\right|\left(\left|R_{i}^{-1}(2 n-1,2 m-1)\right|+\left|R_{i}^{-1}(2 n-1,2 m)\right|\right)\right. \\
& \left.+\sum_{n=1}^{m}\left|R_{i}(2 j-1,2 n)\right|\left(\left|R_{i}^{-1}(2 n, 2 m-1)\right|+\left|R_{i}^{-1}(2 n, 2 m)\right|\right)\right][1+o(1)] \\
\leq & \rho_{i}^{s}\|\xi\|_{\infty} 2\left[\sum_{n=1}^{2} \frac{\left|\mu_{i n 1}^{j-1}\right|}{4\left|\mu_{i 11}^{m-1}\right|} \varepsilon^{(j-m) / 2}+\sum_{n=3}^{m} \frac{\left|\vartheta_{n 1}^{j-1}\right|}{2\left|\mu_{i 11}^{2}\right|} \frac{\left|\operatorname{det} V_{n}\right|}{\left|\operatorname{det} V_{0}\right|} \varepsilon^{j-(m-1)}\right][1+o(1)] .
\end{aligned}
$$

and the conclusion follows by noticing that $j-(m-1)<\frac{j-m}{2}$ for $j<m-2, j-(m-1)=\frac{j-m}{2}$ for $j=m-2$ and $j-(m-1)>\frac{j-m}{2}$ for $j=m-1, m$, as well as $\left|\mu_{i 11}\right|=\sqrt{\lambda_{i} / 2}, \frac{\left|\operatorname{det} V_{n}\right|}{\left|\operatorname{det} V_{0}\right|}=$ $\prod_{3 \leq k \leq m, k \neq n}\left|\vartheta_{k 1}-\vartheta_{n 1}\right|$.

Proof of Lemma 5.5. The proof of Lemma 5.5 relies on the following combinatorial identity.

Lemma 5.7. 116 Let $f(t)=\sum_{k=0}^{\infty} f_{k} t^{k}$ be a formal power series 115. Then the following rule holds if $b=0$ and $f(t)$ is a polynomial:

$$
\sum_{k} C(n+a k, m+b k) z^{m+b k} f_{k}=\left[t^{m}\right](1+z t)^{n} f\left(t^{-b}(1+z t)^{a}\right)
$$

where $\left[t^{m}\right] g(t)$ denotes the extraction of the coefficient of $t^{m}$ from the formal power series $g(t)$. 
Let us return to the proof. Denoting

$$
\begin{aligned}
v(\theta) & =\cos \theta A^{2 m-1}(1, \cdot)+\sin \theta A^{2 m-1}(2, \cdot)+A^{2 m-1}(3, \cdot) \\
& =\left[v_{1}(\theta), v_{2}(\theta), \ldots, v_{2 m-1}(\theta), v_{2 m}(\theta)\right]
\end{aligned}
$$

and recalling $S=A^{2 m-1} \mathcal{O}^{-1}$, the original equation is equivalent to $\pi(\theta) \mathcal{O}=v(\theta)$. Direct computation shows that the entries of $v(\theta)$ are given by

$$
\begin{aligned}
v_{2 j-1}(\theta) & =C(2 m, j-1) \cos (2 m-j+1) \theta, \\
v_{2 j}(\theta) & =C(2 m, j-1) \sin (2 m-j+1) \theta, j=1, \ldots, m ;
\end{aligned}
$$

and the entries of $\mathcal{O}$ are given by

$$
\begin{aligned}
\mathcal{O}(k, 2 j-1) & =C(k-1, j-1) \cos (k-j) \theta, \\
\mathcal{O}(k, 2 j) & =C(k-1, j-1) \sin (k-j) \theta, k=1, \ldots, 2 m, j=1, \ldots, m .
\end{aligned}
$$

As a result, the equation $\pi(\theta) \mathcal{O}=v(\theta)$ is equivalent to the following $m$ equations:

$$
\sum_{k=0}^{2 m-1} \pi_{k}(\theta) C(k, h) e^{\jmath(k-h) \theta}=C(2 m, h) e^{\jmath(2 m-h) \theta}, h=0,1, \ldots, m-1,
$$

or equally

$$
\sum_{k=0}^{2 m} \pi_{k}(\theta) C(k, h) e^{\jmath(k-h) \theta}=0, h=0,1, \ldots, m-1
$$

if we let $\pi_{2 m}(\theta)=-1$. Noticing that $2 \cos \theta=e^{\jmath \theta}+e^{-\jmath \theta}$, we substitute the expression of $\pi_{k}(\theta)$ into the left-hand side of the above $h$-th equation, and expand it into a power series of $e^{\jmath \theta}$ as $\sum_{k=0}^{2 m} \pi_{k}(\theta) C(k, h) e^{\jmath(k-h) \theta}=\sum_{w=0}^{m} \alpha_{w, h} e^{\jmath(2 w-h) \theta}$, with

$$
\alpha_{w, h}=\sum_{k=0}^{w} \sum_{j=w-k}^{m-k} C(m, j) C(m-j, k) C(j, w-k) C(j+2 k, h)(-1)^{j+2 k-1} .
$$

Therefore, if we can show that $\alpha_{w, h}$ for $w=0,1, \ldots, m$ and $h=0,1, \ldots, m-1$, then the prescribed $\pi(\theta)$ is a solution of 5.64 , and by the nonsigularity of $\mathcal{O}$ it is also unique.

We first transform $\alpha_{w, h}$ as follows. By considering that

$$
\begin{aligned}
C(m, j) C(m-j, k) C(j, w-k) & =C(m, k) C(m-k, j) C(j, w-k) \\
& =C(m, k) C(m-k, w-k) C(m-w, j-(w-k))
\end{aligned}
$$


and letting $s=j-(w-k)$, it is clear that

$$
\alpha_{w, h}=\sum_{k=0}^{w} C(m, k) C(m-k, w-k)(-1)^{k+w-1} \sum_{s=0}^{m-w} C(m-w, s) C(s+w+k, h)(-1)^{s} .
$$

Now we claim that

1) $\sum_{s=0}^{m-w} C(m-w, s) C(s+w+k, h)(-1)^{s}=C(w+k, h-(m-w))(-1)^{m-w}$, $w=0,1, \ldots, m$,

2) $\sum_{k=0}^{w} C(m, k) C(m-k, w-k) C(w+k, h-(m-w))(-1)^{m+k-1}=0$, $h=0,1, \ldots, m-1$,

and the proof of the first part is completed by combining these two claims.

1) Let $f_{s}=(-1)^{s} C(m-w, s)$ and $f(t)=\sum_{s} f_{s} t^{s}=(1-t)^{m-w}$. Applying Lemma 5.7 we have

$$
\begin{aligned}
& \sum_{s=0}^{m-w} C(m-w, s) C(s+w+k, h)(-1)^{s} \\
= & \sum_{s=0}^{m-w} C(w+k+1 \cdot s, h+0 \cdot s) \cdot 1 \cdot f_{s} \\
= & {\left[t^{h}\right](1+t)^{w+k} f(1+t) } \\
= & {\left[t^{h}\right](1+t)^{w+k}(-t)^{m-w} } \\
= & (-1)^{m-w}\left[t^{h-(m-w)}\right](1+t)^{w+k} \\
= & (-1)^{m-w} C(w+k, h-(m-w)),
\end{aligned}
$$

which establishes the first claim.

2) For the second claim,

$$
\begin{aligned}
& \sum_{k=0}^{w} C(m, k) C(m-k, w-k) C(w+k, h-(m-w))(-1)^{m+k-1} \\
= & (-1)^{m-1} \sum_{k=0}^{m} C(m, k)(-1)^{k} C(m-k, w-k) C(w+k, h-(m-w)) \\
= & (-1)^{m-1} \sum_{k=0}^{m}\left[t^{k}\right](1-t)^{m}\left[v^{m-w}\right](1+v)^{m-k}\left[u^{h-(m-w)}\right](1+u)^{w+k} \\
= & (-1)^{m-1}\left[v^{m-w}\right](1+v)^{m}\left[u^{h-(m-w)}\right](1+u)^{w} \sum_{k=0}^{m}\left[t^{k}\right]\left(\frac{1+u}{1+v}\right)^{k}(1-t)^{m} \\
= & (-1)^{m-1}\left[v^{m-w}\right](1+v)^{m}\left[u^{h-(m-w)}\right](1+u)^{w}\left(1-\frac{1+u}{1+v}\right)^{m} \\
= & (-1)^{m-1}\left[v^{m-w}\right](1+v)^{m}\left[u^{h-(m-w)}\right](1+u)^{w} \frac{(v-u)^{m}}{(1+v)^{m}}
\end{aligned}
$$




$$
\begin{aligned}
& =(-1)^{m-1}\left[v^{m-w}\right]\left[u^{h-(m-w)}\right](1+u)^{w}(v-u)^{m} \\
& \stackrel{\underline{r=h-(m-w)}}{=}(-1)^{m-1}\left[v^{m-w}\right]\left[u^{r}\right](1+u)^{w}(v-u)^{m} \\
& =(-1)^{m-1}\left[v^{m-w}\right] \sum_{k=0}^{r} C(w, r-k) C(m, k) v^{m-k}(-1)^{k} \\
& =(-1)^{m-1}\left[v^{k-w}\right] \sum_{k=0}^{r} C(w, r-k) C(m, k)(-1)^{k},
\end{aligned}
$$

where $t, v, u$ are indeterminates. Noticing that $r \geq w \Leftrightarrow h-(m-w) \geq w \Leftrightarrow h \geq m$ is contradictory to $h=0,1, \ldots, m-1$, we have $r<w$ and $k \leq r<w$, which suggests the vanishing of the last equation in the above, and the proof for the first part is complete.

As for the second part, by noting that the exponents of $\cos \theta$ in $\pi_{k}(\theta)$ are even when $k$ is an even number, while the exponents are odd when $k$ is an odd number, it can be noted that the sign of each term in $\pi_{k}(\theta)$ is the same. Therefore we obtain

$$
\sum_{k=0}^{2 m-1}\left|\pi_{k}(\theta)\right|=\sum_{k=0}^{2 m-1} \sum_{h=0}^{\lfloor k / 2\rfloor} C(m, k-2 h) C(m-(k-2 h), h)|2 \cos \theta|^{k-2 h}=\sum_{j=0}^{m} \beta_{j}|\cos \theta|^{j}
$$

with $\beta_{0}=2^{m}-1$ and $\beta_{j}=2^{j} C(m, j) \sum_{h=0}^{m-j} C(m-j, h)=2^{m} C(m, j)$ for $j=1, \ldots, m$, and the conclusion follows directly. 



\section{Part II}

\section{Optimal Consensus}

\section{under Set or Kinematic Constraints}




\section{Chapter 6}

\section{Continuous-time Optimal \\ Consensus under Set Constraint}

In this chapter, we study a distributed optimal consensus problem of continuous-time MASs under a common set constraint. Each agent is assigned with an individual cost function which is coercive and convex, and is required to reach the minimum of the aggregate cost within the given constraint set. To this end, three terms are included in the control protocol: local averaging, local projection, and local subgradient descent with a diminishing gain $\alpha(t)$. Under a balanced communication network with uniformly joint spanning trees and bounded time-varying edge weights, we show that the constrained optimal consensus can be achieved when $\alpha(t)$ satisfies a persistence condition. When the aggregate cost is strongly convex, we further provide a systematic convergence rate analysis for different types of $\alpha(t)$, and choose the best step size for the fastest convergence.

The rest of the chapter is organized as follows. The problem is first formulated in Section 6.1 followed by the control protocol and the convergence result presented in Section 6.2. The complete convergence analysis is conducted in Section 6.3 The convergence rate analysis for strongly convex aggregate functions is provided in Section 6.4. Numerical examples are given in Section 6.5, and we conclude the chapter in Section 6.6

\subsection{Problem Formulation}

Consider an MAS of continuous-time dynamics, with each agent $i$ assuming a state value $x_{i} \in \mathbb{R}^{m}$ and a local cost function $f_{i}\left(x_{i}\right)$. Given the first order dynamics

$$
\dot{x}_{i}(t)=u_{i}(t), i=1, \ldots, N,
$$


we shall design the control input $u_{i}$ in a distributed way to guarantee the consensus in a constraint set $\mathcal{X}$, and meanwhile minimize the aggregate cost as a sum of individual costs. In summary, to achieve the distributed constrained optimal consensus is equivalent to solving the following problem:

$$
\min \sum_{i} f_{i}\left(x_{i}\right), \text { s.t. } x_{1}=\cdots=x_{N}, x_{i} \in \mathcal{X}
$$

Moreover, assume that

Assumption 6.1. $\mathcal{X}$ is closed and convex.

Assumption 6.2. For each $i, f_{i}: \mathbb{R}^{m} \rightarrow \mathbb{R}$ is convex and coercive.

Denote $F(x)=\sum_{i} f_{i}(x)$. By Assumptions 6.1 and 6.2 the optimum set of $F$ within $\mathcal{X}$ must be convex and compact, and we denote it as $\mathcal{X}^{*}$. Therefore, 6.1) is solved asymptotically if we can design a distributed control protocol such that

$$
\begin{gathered}
\lim _{t \rightarrow \infty}\left(x_{i}(t)-x_{j}(t)\right)=0, i, j=1, \ldots, N, \\
\lim _{t \rightarrow \infty}\left|x_{i}(t)\right|_{\mathcal{X}^{*}}=0, i=1, \ldots, N .
\end{gathered}
$$

Remark 6.1. We illustrate the problem by a relevant example of optimal constrained rendezvous. To be specific, with $f_{i}\left(x_{i}\right)=\left\|x_{i}-x_{i}(0)\right\|$ in (6.1), each agent is to approach $x^{*} \in \mathcal{X}$ such that $\sum_{i}\left\|x^{*}-x_{i}(0)\right\|$ is minimized. Note that when there is no set constraint, the rendezvous location is actually the geometric median of the original positions of UAVs. Furthermore, the uniqueness of $x^{*}$ comes from the strict convexity of distance functions.

\subsection{Control Design and Convergence Result}

In this section we shall devise a control protocol for the constrained optimal consensus and present a convergence result. Based on subgradients and projection, the control input is proposed as

$$
\begin{aligned}
u_{i} & =\sum_{j} a_{i j}(t)\left(x_{j}-x_{i}\right)-\alpha\left(t+\tau_{i}\right) s_{i}\left(x_{i}\right)+P_{\mathcal{X}}\left(x_{i}\right)-x_{i} \\
& \in \sum_{j} w_{i j}(t)\left(x_{j}-x_{i}\right)-\alpha\left(t+\tau_{i}\right) \partial f_{i}\left(x_{i}\right)+P_{\mathcal{X}}\left(x_{i}\right)-x_{i} \\
& \triangleq G_{i}\left(t, x_{1}, \ldots, x_{N}\right),
\end{aligned}
$$

and the system dynamics becomes

$$
\dot{x}_{i} \in G_{i}\left(t, x_{1}, \ldots, x_{N}\right), i=1, \ldots, N
$$


where $s_{i}\left(x_{i}\right) \in \partial f_{i}\left(x_{i}\right), w_{i j}(t)$ is a time-varying weight on communication edge $(i, j)$, and $\alpha(t)>0$ is a continuous time-varying gain. Note that we introduce a piecewise continuous function $\tau_{i}$ to model the asynchronization among different agents, and we assume that $\tau_{i}(t) \in[0, \bar{\tau}]$ which can be satisfied by time synchronization during every fixed time interval.

Remark 6.2. To achieve the optimal consensus within the given constraint $\mathcal{X}$, three terms have been included into the control input (6.3) respectively as local averaging for consensus, local projection for approaching the set constraint, and local subgradient descent for minimizing the aggregate cost. The additional time-varying gain is to address the imbalance between the constrained consensus and optimization, which will be discussed in more details in Section 6.3. In comparison with the update protocol in distributed optimization literature [69 71], we combine three terms into a single step, instead of separating the local averaging and the local projected subgradient step. Moreover, unlike the above works where the set constraint is satisfied by projecting the intermediate update onto $\mathcal{X}$, here the asymptotic approaching of $\mathcal{X}$ relies on a movement step along the projection direction in 6.3, which is more suitable for mobile systems.

Remark 6.3. In general, the projection cannot be solved in closed-form even when $\mathcal{X}$ is explicitly given and an optimization algorithm is required. But in the case of set constraint, $\mathcal{X}$ is usually given as a sphere or convex polytope. In the former case, a real-time projection is readily achieved. In the latter one, we note that once the faces containing projection point are determined, say $h_{x i}^{\prime} y=b_{x i}, i=$ $1, \ldots, M$, then the projection can be found immediately by solving an equality-constrained problem as below:

$$
\min _{y} \frac{1}{2}\|x-y\|^{2}, \text { s.t. } h_{x i}^{\prime} y=b_{x i}, i=1, \ldots, M \text {. }
$$

By the method of Lagrange multipliers, $P_{\mathcal{X}}(x)=x^{*}$ can be found by the solution to the following equation

$$
\left[\begin{array}{cc}
I & \tilde{H}_{x}^{\prime} \\
\tilde{H}_{x} & 0
\end{array}\right]\left[\begin{array}{l}
x^{*} \\
\lambda^{*}
\end{array}\right]=\left[\begin{array}{c}
x \\
\tilde{b}_{x}
\end{array}\right],
$$

where $\tilde{H}_{x}$ and $\tilde{b}_{x}$ are respectively comprised of the rows $h_{x i}^{\prime}$ 's and $b_{x i}$ 's. Note that $\tilde{H}_{x}$ has only finitely different forms dependent on the number of faces, and the symmetric and indefinite coefficient matrix in (6.5) can be effectively factorized by Bunch-Parlett method [117]. Thus, if we can partition the whole space into different sets such that the points in the same set project to the same face of the polytope, then the projection of points in the same set is readily achieved by 6.5. Given the polytope $\mathcal{X}$, the partition and the corresponding factorization may be obtained in advance, and for the purpose of real-time optimization it mainly needs to choose the partition set for the point $x$. Although the partition and selecting the set may be not so easy in general cases when the polytope contains a large 
number of faces, the real-time projection is still feasible if the polytope only contains a few faces. For instance, assume that $\mathcal{X}$ is a triangle on the plane with vertexes $(0,0),(0,1)$ and $(1,0)$. Let $x=\left(x_{1}, x_{2}\right)$ be a point on the plane. It follows that

$$
P_{\mathcal{X}}(x)= \begin{cases}x, & x \in \mathcal{X} \\ \left(0, x_{2}\right), & x_{1}<0,0<x_{2} \leq 1 \\ (0,1), & x_{1} \leq x_{2}-1, x_{2}>1 \\ \left(x_{1}, 0\right), & x_{1}-1 \leq x_{2}<x_{1}+1, x_{2}>-x_{1}+1 \\ (1,0), & x_{1} \geq 1, x_{2}<x_{1}-1 \\ \frac{\left(x_{1}, x_{2}\right)}{\left(x_{1}+x_{2}\right)}, & 0 \leq x_{1}<1, x_{2}<0 \\ (0,0), & x_{1}<0, x_{2} \leq 0\end{cases}
$$

The following assumptions are respectively about the switching frequency of time-varying communication network $\mathcal{G}$ and the time-varying edge weights $w_{i j}(t)$.

Assumption 6.3. $\mathcal{G}$ is piecewise constant, and $t_{k+1}-t_{k} \geq \tau_{D}>0$ for any two consecutive switching instants $t_{k}$ and $t_{k+1}$.

Assumption 6.4. $0<w_{*} \leq w_{i j}(t) \leq w^{*}$ whenever $w_{i j}(t)>0$.

Remark 6.4. Assumption 6.3 dictates that the communication network cannot change too fast, which is quite common in practice. Assumption 6.4 requires the positive edge weight to be upper and lower bonded by positive constants, which is also easily satisfied.

The following two assumptions are about the time-varying gain $\alpha(t)$.

Assumption 6.5. $\int_{t_{0}}^{\infty} \alpha(t) d t=\infty$.

Assumption 6.6. $\lim _{t \rightarrow \infty} \frac{\dot{\alpha}(t)}{\alpha(t)}=0$.

Now the main result is presented as follows.

Theorem 6.1. Suppose Assumptions 6.1 and 6.2 hold. Then the constrained optimal consensus problem 6.1 can be asymptotically solved by the distributed control protocol (6.3) with a diminishing $\alpha(t)$, i.e. $\lim _{t \rightarrow \infty} \alpha(t)=0$, if the following conditions are further satisfied:

1) The communication graph $\mathcal{G}$ stays balanced and uniformly contains joint spanning trees, and Assumptions 6.3 and 6.4 are satisfied;

2) $\alpha(t)$ satisfies Assumptions 6.5 and 6.6 . 
Remark 6.5. Note that the global minimizer may lie outside the constraint set which causes a conflict between the projection term and subgradient term. Thus, we introduce the scaling function $\alpha(t)$ which diminishes with time to ensure the convergence in the constraint set. Additionally, the subgradient effect should be persistent enough to reach the constrained optimal value, as required by the persistence condition 6.5. Here we only require $\alpha(t)$ to be non-integrable, in contrast to the case of being nonsummable but square summable in many existing works e.g. [69, 118]. A diminishing $\alpha(t)$ is also somewhat necessary as shown in [119]: assuming $\alpha(t)$ to be constant, the constrained optimization problem can only be solved when the intersection of the minimum sets of each $f_{i}$ is non-empty.

Remark 6.6. Assumption 6.6 is only used for mitigating the asynchronization effect. It is a mild condition and can be easily satisfied by many functions, e.g. $\alpha(t)=1 / t$ or $\alpha(t)=1 /[t \ln (t)]$. In fact, it dictates a change rate much slower than $\alpha(t)$, which is consistent with the persistence condition: if $\limsup _{t \rightarrow \infty} \frac{\dot{\alpha}(t)}{\alpha(t)}=c<0$, then $\alpha(t) \leq \alpha\left(t_{0}\right) e^{c\left(t-t_{0}\right)}$ and the persistence condition 6.5 no longer holds. With Assumption 6.6 , it is easy to check that there exists $\varepsilon(t) \geq 0$ such that $e^{-\varepsilon(t) \tau_{i}} \leq \frac{\alpha\left(t+\tau_{i}\right)}{\alpha(t)} \leq e^{\varepsilon(t) \tau_{i}}$ with $\lim _{t \rightarrow \infty} \varepsilon(t)=0$. Consequently, it holds that

$$
\left|\alpha\left(t+\tau_{i}\right)-\alpha(t)\right| \leq\left(e^{\varepsilon(t) \tau_{i}}-1\right) \alpha(t) \leq \varepsilon(t) \bar{\tau} \alpha(t), \tau_{i} \in[0, \bar{\tau}]
$$

\subsection{Convergence Analysis}

It is clear from 6.3 that three different kinds of 'forces', consisting of consensus, optimization (subgradient) and set projection, are applied to the state value of each node. The convergence analysis is challenging with these forces jointly in effect. Also note that the time-varying dynamics excludes the existence of Lyapunov functions, and in general the optimum set $\mathcal{X}^{*}$ is not an equilibrium set (e.g. consider the single agent case with $\left.F(x)=(x-2)^{2}, \mathcal{X}=(-\infty, 0]\right)$. However, the time-varying gain $\alpha(t)$ creates an imbalance among the forces, and allows us to first establish the consensus within the constraint set, and then achieve the constrained optimization. To be more specific, once the solution has been found as globally bounded, the local averaging and local projection will dominate over the diminishing subgradient and yield the convergence to the constraint set for all states, while the persisting subgradient force ensures the constrained optimization. In the sequel the convergence analysis proceeds along the exact line: the global boundedness of the solution, constraint set convergence, consensus and optimum set convergence. Before moving to the formal analysis, we first review some preliminaries on the solution to differential equations with discontinuous righthand sides. 


\subsubsection{Preliminaries on Differential Equations with Discontinuous Right- hand Sides}

For a differential equation $\dot{x}(t)=f(t, x)$, where $f(t, x)$ may not be continuous everywhere, a solution to the above equation in the sense of Caratheodory is an a.c. function defined on $\left[t_{0}, t_{d}\right]$ which satisfies $\dot{x}(t)=f(t, x)$ a.e. A well known theorem about the existence and uniqueness of the solution is stated as follows:

Lemma 6.1. [120] Given $a, b>0$, let $f(t, x)$ be defined on $\left\{(t, x): t \in\left[t_{0}, t_{0}+a\right],\left\|x-x_{0}\right\| \leq b\right\}$ and satisfy the Caratheodory's condition:

1. $f(t, x)$ is continuous in $x$ for almost all $t$;

2. $f(t, x)$ is measurable in $t$ for each $x$;

3. $\|f(t, x)\| \leq \mathcal{F}(t)$ and $\mathcal{F}(t)$ is locally integrable, i.e. integrable on every finite interval.

Then there exists a solution on a closed interval $\left[t_{0}, t_{0}+d\right]$, where $d \leq a$ is selected such that $\int_{t_{0}}^{t_{0}+d} \mathcal{F}(t) d t \leq b$. Furthermore, if $\langle f(t, x)-f(t, y), x-y\rangle \leq \mathcal{L}(t)\|x-y\|^{2}$ with $\mathcal{L}(t)$ locally integrable, then the solution is unique.

On the other hand, for a set-valued map $G(t, x)$ defined on a region $E$, a solution $x(t)$ to the differential inclusion

$$
\dot{x}(t) \in G(t, x)
$$

is an a.c. function such that 6.7 is a.e. satisfied on $\left[t_{0}, t_{d}\right]$, with initial value $x\left(t_{0}\right) \in E$. A commonly used theorem about the local existence of the solution is given as follows:

Lemma 6.2. [120] Let $G(t, x)$ be a nonempty compact convex set and upper semi-continuous for any $(t, x)$ in some domain $E$. If $E$ contains the cylinder $Z=\left\{(t, x): t \in\left[t_{0}, t_{0}+a\right],\left\|x-x_{0}\right\| \leq\right.$ $b\}$, then (6.7) has a solution $x(t)$ on $\left[t_{0}, t_{0}+d\right]$ with $x\left(t_{0}\right)=x_{0}$, where $d=\min \{a, b / \bar{G}\}, \bar{G}=$ $\sup _{(t, x) \in Z}\|G(t, x)\|$. Similarly, the solution exists on $\left[t_{0}-d^{\prime}, t_{0}\right]$ if $E$ contains the cylinder $Z^{\prime}=$ $\left\{(t, x): t \in\left[t_{0}-a^{\prime}, t_{0}\right],\left\|x-x_{0}\right\| \leq b^{\prime}\right\}$, where $d^{\prime}=\min \left\{a^{\prime}, b^{\prime} / \bar{G}\right\}, \bar{G}^{\prime}=\sup _{(t, x) \in Z^{\prime}}\|G(t, x)\|$.

The following Dini derivatives are needed to study the solution to a differential inclusion:

$$
\begin{aligned}
& D^{+} x(t)=\limsup _{h \rightarrow 0^{+}} \frac{1}{h}[x(t+h)-x(t)], \\
& D_{+} x(t)=\liminf _{h \rightarrow 0^{+}} \frac{1}{h}[x(t+h)-x(t)] .
\end{aligned}
$$


The other two types of Dini derivatives, $D^{-} x(t)$ and $D_{-} x(t)$, can be respectively obtained by letting $h \rightarrow 0_{-}$in the above equations. By the following lemma, we can obtain the Dini derivatives from the set-valued map $G(t, x)$.

Lemma 6.3. [120] Let the conditions of Lemma 6.2 hold. Then it holds that

$$
\operatorname{Cont}(x(t)) \subset G(t, x(t)), t \in\left(t_{0}, t_{0}+d\right)
$$

where $\operatorname{Cont}(x(t))=\left\{\alpha: \alpha=\lim _{k \rightarrow \infty} \frac{x\left(\tau_{k}\right)-x(t)}{\tau_{k}-t}\right\}$ and $\left\{\tau_{k}\right\}_{k=1}^{\infty}$ is any sequence of points with $\lim _{k \rightarrow \infty} \tau_{k}=t$. Specifically, for any given Dini derivative $D, D x(t) \in \operatorname{Cont}(x(t))$.

The comparison principle is also useful in the subsequent analysis.

Lemma 6.4 (Comparison Principle 121]). Let $E$ be an open set in $\mathbb{R}^{2}$ and $f(t, u)$ be a continuous scalar function defined on $E$. Assume that $\mu(t)$ and $\eta(t)$ are continuous on $\left[t_{0}, t_{0}+a\right)$, with $(t, \mu(t))$, $(t, \eta(t)) \in E$. Suppose further that $v\left(t_{0}\right) \leq w\left(t_{0}\right)$ and the inequalities below hold for $t \in\left(t_{0}, t_{0}+a\right)$ :

$$
D \mu(t)<f(t, \mu(t)), D \eta(t) \geq f(t, \eta(t)),
$$

where $D$ is any fixed Dini derivative. Then $\mu(t)<\eta(t), t \in\left(t_{0}, t_{0}+a\right)$.

\subsubsection{Globally Bounded Solution}

In this subsection we shall show that the solution $x_{i}(t)$ of 6.4 is globally bounded and therefore exists over $\left[t_{0}, \infty\right)$. The following result is needed.

Lemma 6.5. Let $\xi_{i}(t), i=1, \ldots, N$ be continuous on $(a, b)$ and $\xi(t)=\max _{i} \xi_{i}(t)$. If $D^{+} \xi_{i}(t) \leq h_{i}(t)$ with $h_{i}$ being upper semi-continuous, then $D^{+} \xi(t) \leq \max _{i \in I(t)}\left\{h_{i}(t)\right\}$, where $I(t)=\left\{i: \xi_{i}(t)=\xi(t)\right\}$.

Proof. It suffices to prove the case of $N=2$. If $I(t)$ consists of a single index, the result naturally holds. Otherwise, given $t \in(a, b)$ and $\varepsilon>0$, let $v_{i}(\tau)=\xi_{i}(\tau)-(h(t)+\varepsilon) \tau$, where $h(t)=\max \left\{h_{1}(t), h_{2}(t)\right\}$. By the upper semi-continuity of $h_{i}(t)$, for some $t_{\varepsilon}>0$ we have $h(\tau) \leq h(t)+\varepsilon$ on $\left(t-t_{\varepsilon}, t+t_{\varepsilon}\right)$. As a result, $D^{+} v_{i}(\tau)=D^{+} \xi_{i}(\tau)-(h(t)+\varepsilon) \leq 0$ on $\left(t-t_{\varepsilon}, t+t_{\varepsilon}\right)$ by the assumption, implying that $v_{i}$ 's are both non-increasing. Therefore, $v(\tau)=\max _{i} v_{i}(\tau)$ is also non-increasing and $D^{+} v(\tau) \leq 0$. Noticing that $v(\tau)=\xi(\tau)-(h(t)+\varepsilon) \tau$, we have $D^{+} \xi(\tau) \leq h(t)+\varepsilon$ on $\left(t-t_{\varepsilon}, t+t_{\varepsilon}\right)$. In particular, $D^{+} \xi(t) \leq h(t)+\varepsilon$. The result is established by letting $\varepsilon \rightarrow 0$.

Remark 6.7. A relevant result can be found in [122], where $D^{+} \xi(t)=\max _{i \in I(t)}\left\{\dot{\xi}_{i}(t)\right\}$. However, in most cases, it is difficult and even impossible to find the change rate of each $\xi_{i}$, while an upper bound of the Dini derivative of the maximum function is enough for the analysis. We can see soon the key role of Lemma 6.5 . 
We now return to the analysis of the solution of 6.4 . Without loss of generality we assume that the switching instants of the $\mathcal{G}$ coincide with the discontinuities of each $\tau_{i}$. The condition about $G\left(t, x_{1}, \ldots, x_{N}\right)$ in Lemma 6.2 is readily met on any time interval $\left[t_{k}, t_{k+1}\right)$ when the switching signal remains constant and each $\tau_{i}$ is continuous; as a result, for any given initial value $\left(x_{10}, \ldots, x_{N 0}\right)$, there always exists $0<a<t_{1}$ such that $x_{i}(t)$ is a.c. on $\left[t_{0}, t_{0}+a\right]$ with $x_{i}\left(t_{0}\right)=x_{i 0}, i=1, \ldots, N$, and satisfies 6.4 almost everywhere. If $x_{i}(t)$ can be continued to the next switching instant $t_{1}$, then it can be similarly continued to $\infty$. Therefore, to establish the global boundedness of the solution, it suffices to show that $x_{i}(t)$ is bounded independently of time $t$ on $\left[t_{0}, t_{1}\right)$, with constant $w_{i j}(t)=w_{i j}$.

For a fixed $z \in \mathcal{X}$, define $d_{z}(x)=\|x-z\|^{2}, \bar{d}_{z}(t)=\max _{i} d_{z}\left(x_{i}(t)\right)$. The fact that $d_{z}(x)$ is locally Lipschitz ensures the absolute continuity of $d_{z}\left(x_{i}(t)\right)$ on any open interval in $\left[t_{0}, t_{0}+a\right]$; in addition, the derivative of $d_{z}\left(x_{i}(t)\right)$ a.e. satisfies that

$$
\begin{aligned}
\dot{d}_{z}\left(x_{i}(t)\right) & =2\left\langle x_{i}-z, \dot{x}_{i}\right\rangle \\
& \in 2\left\langle x_{i}-z, \sum_{j} w_{i j}\left(x_{j}-x_{i}\right)-\alpha\left(t+\tau_{i}\right) \partial f_{i}\left(x_{i}\right)+P_{\mathcal{X}}\left(x_{i}\right)-x_{i}\right\rangle
\end{aligned}
$$

We are to show that there exists a constant $R_{0}$ such that $D^{+} \bar{d}_{z}(t)<0$ whenever $\bar{d}_{z}(t) \geq R_{0}$, and conclude that $\left\|x_{i}(t)-z\right\| \leq \max \left\{R_{0}, \max _{j}\left\|x_{j}\left(t_{0}\right)-z\right\|\right\}$.

To this end, we first observe that $\left\langle\partial f_{i}\left(x_{i}\right), x_{i}-z\right\rangle>0$ when $x_{i}$ is far from $z$. Actually, denote $\phi_{i, x_{i}}(t)=f_{i}\left(z+t\left(x_{i}-z\right)\right)$, then we have $\partial \phi_{i, x_{i}}(1)=\left\langle\partial f_{i}\left(x_{i}\right), x_{i}-z\right\rangle$ by Lemma 3.8 From the definition of subdifferential it is easy to see that for the scalar function $\phi_{i, x_{i}}(t)$, all the numbers in $\partial \phi_{i, x_{i}}(t)$ are positive if $\phi_{i, x_{i}}$ is strictly increasing around $t$. Moreover, being convex, $\phi_{i, x_{i}}$ is strictly increasing at $t=1$ if $\phi_{i, x_{i}}(1)>\phi_{i, x_{i}}(0)$, or equally $f_{i}\left(x_{i}\right)>f_{i}(z)$. With $f_{i}\left(x_{i}\right)$ being coercive, there always exists a sufficiently large constant $R_{0}$ such that $f_{i}\left(x_{i}\right)>f_{i}(z)$ whenever $\left\|x_{i}-z\right\| \geq R_{0}$, and $\left\langle\partial f_{i}\left(x_{i}\right), x_{i}-z\right\rangle>0$ for such $x_{i}$.

With the above preparation, we are to study $D^{+} \bar{d}_{z}(t)$ for $\bar{d}_{z}(t) \geq R_{0}$. Denoting $h_{i}\left(x_{i}\right)=$ $-\min _{s \in \partial f_{i}\left(x_{i}\right)}\left\langle x_{i}-z, s\right\rangle$, the upper semi-continuity of $h_{i}\left(x_{i}\right)$ can be inferred from that of $\partial f_{i}\left(x_{i}\right)$. Furthermore, by recalling 6.8 and Lemma 6.3 it is readily seen that

$$
\begin{aligned}
D^{+} d_{z}\left(x_{i}(t)\right) \leq & 2\left\langle x_{i}(t)-z, \sum_{j} w_{i j}\left(x_{j}(t)-x_{i}(t)\right)\right\rangle \\
& +2 \alpha\left(t+\tau_{i}\right) h_{i}\left(x_{i}(t)\right)-2\left|x_{i}(t)\right|_{\mathcal{X}}^{2} \\
\leq & 2\left\langle x_{i}(t)-z, \sum_{j} w_{i j}\left(x_{j}(t)-x_{i}(t)\right)\right\rangle+2 \alpha\left(t+\tau_{i}\right) h_{i}\left(x_{i}(t)\right) .
\end{aligned}
$$

Now in the light of Lemma 6.5 and $\left\langle x_{i}(t)-z, x_{j}(t)-x_{i}(t)\right\rangle \leq 0$ for any $j$ when $i \in I_{d}(t)=\{i$ : 
$\left.d_{z}\left(x_{i}(t)\right)=\bar{d}_{z}(t)\right\}$, it yields that

$$
D^{+} \bar{d}_{z}(t) \leq \max _{i \in I(t)}\left\{2 \alpha\left(t+\tau_{i}\right) h_{i}\left(x_{i}(t)\right)\right\}<0,
$$

suggesting a decreasing $\bar{d}_{z}(t)$ when $\bar{d}_{z}(t) \geq R_{0}$. Thus, we know that the solution is globally bounded.

\subsubsection{Constraint Set Convergence}

In this subsection we shall prove that

$$
\lim _{t \rightarrow \infty}\left|x_{i}(t)\right|_{\mathcal{X}}=0, i=1, \ldots, N
$$

Noticing that the solution is globally bounded and recalling the upper semi-continuity of subdifferentials from Lemma 3.7. we can find a constant $\bar{\gamma}>0$ such that

$$
\max _{s_{i}(t) \in \partial f_{i}\left(x_{i}(t)\right)}\left\|s_{i}(t)\right\| \leq \bar{\gamma}, \forall i, t \geq t_{0}
$$

Denote $p_{i}\left(x_{i}\right)=\left|x_{i}\right|_{\mathcal{X}}^{2}$ and $p(t)=\max _{i} p_{i}\left(x_{i}(t)\right)$. Since $p_{i}$ is locally Lipschitz, $p_{i}\left(x_{i}(t)\right)$ is locally a.c. and its derivative a.e. satisfies

$$
\begin{aligned}
\dot{p}_{i}= & 2\left\langle x_{i}(t)-P_{\mathcal{X}}\left(x_{i}(t)\right), \dot{x}_{i}(t)\right\rangle \\
\in & 2\left\langle x_{i}(t)-P_{\mathcal{X}}\left(x_{i}(t)\right), \sum_{j} w_{i j}(t)\left(x_{j}(t)-x_{i}(t)\right)\right. \\
& \left.-\alpha\left(t+\tau_{i}\right) \partial f_{i}\left(x_{i}(t)\right)+P_{\mathcal{X}}\left(x_{i}(t)\right)-x_{i}(t)\right\rangle .
\end{aligned}
$$

Invoking Lemma 3.6, Lemma 6.3 and 6.10, it holds everywhere that

$$
\begin{aligned}
D^{+} p_{i} \leq & 2 \sum_{j} w_{i j}(t)\left|x_{i}(t)\right|_{\mathcal{X}}\left(\left|x_{j}(t)\right|_{\mathcal{X}}-\left|x_{i}(t)\right|_{\mathcal{X}}\right) \\
& +2 \alpha\left(t+\tau_{i}\right) \bar{\gamma}\left|x_{i}(t)\right| \mathcal{X}-2\left|x_{i}(t)\right|_{\mathcal{X}}^{2}
\end{aligned}
$$

if $t$ is not a switching instant, i.e. $\mathcal{G}(t)$ remains constant in a neighborhood of $t$. Otherwise consider

$$
z_{i} \in \sum_{j} w_{i j}\left(t^{*}\right)\left(z_{j}-z_{i}\right)-\alpha\left(\tau+\tau_{i}\right) \partial f_{i}\left(z_{i}\right)+P_{\mathcal{X}}\left(z_{i}\right)-z_{i}
$$

with initial value $z_{i}\left(t^{*}\right)=x_{i}\left(t^{*}\right)$ for $i=1, \ldots, N$. By Lemma 6.2 we obtain a local solution $z_{i}(\tau)$ over $\left[t^{*}-a, t^{*}\right]$. Now define with sufficiently small $\varepsilon$ that

$$
\tilde{x}_{i}(\tau)= \begin{cases}z_{i}(\tau), & \tau \in\left[t^{*}-\varepsilon, t^{*}\right) \\ x_{i}(\tau), & \tau \in\left[t^{*}, t^{*}+\varepsilon\right] .\end{cases}
$$


We can estimate $\tilde{x}_{i}(\tau)$ similar to 6.12); in particular, noticing that $\tilde{x}_{i}(\tau)=x_{i}(\tau)$ for $\tau \in\left[t^{*}, t^{*}+\varepsilon\right]$, 6.12 holds at $t=t^{*}$ and eventually it holds for every $t$.

Moreover, for any $i \in I_{p}(t)=\left\{i: p_{i}\left(x_{i}(t)\right)=p(t)\right\}$,

$$
D^{+} p_{i} \leq-2 p(t)+2 \alpha\left(t+\tau_{i}\right) \bar{\gamma} \sqrt{p(t)}
$$

by virtue of $\left|x_{j}(t)\right|_{\mathcal{X}}-\left|x_{i}(t)\right|_{\mathcal{X}} \leq 0$ for any $j$ in 6.12 . Combining Lemma 6.5 we attain

$$
D^{+} p(t) \leq-2 p(t)+2 \max _{i \in I} \alpha\left(t+\tau_{i}\right) \bar{\gamma} \sqrt{p(t)}
$$

If we note that for any $\delta>0$, there exists a time constant $t_{\delta}$ such that $\forall t \geq t_{\delta}, \alpha\left(t+\tau_{i}\right)<\delta, 6.13$ becomes

$$
D^{+} p(t)<-2 p(t)+2 \delta \bar{\gamma} \sqrt{p(t)}, t \geq t_{\delta}
$$

The solution of the ODE $\left\{\begin{array}{l}\dot{y}(t)=2(-y+\delta \bar{\gamma} \sqrt{y}) \\ y\left(t_{\delta}\right)=p\left(t_{\delta}\right)+\delta>0\end{array}\right.$ is found as

$$
y(t)=\left[\delta \bar{\gamma}+e^{-\left(t-t_{\delta}\right)}\left(\sqrt{y\left(t_{\delta}\right)}-\delta \bar{\gamma}\right)\right]^{2}
$$

and by the comparison principle 6.4 we obtain

$$
p(t)<\left[\delta \bar{\gamma}+e^{-\left(t-t_{\delta}\right)}\left(\sqrt{p\left(t_{\delta}\right)+\delta}-\delta \bar{\gamma}\right)\right]^{2}, t \geq t_{\delta}
$$

suggesting that $\limsup _{t \rightarrow \infty} p(t) \leq(\delta \bar{\gamma})^{2}$. The conclusion is reached by the arbitrariness of $\delta$.

\subsubsection{Consensus}

In this subsection we shall prove 6.2a). With the constraint set convergence and the diminishing $\alpha(t)$, the collective dynamics can be seen as a linear system with diminishing disturbance. Observe that the issue of time-varying graph is usually addressed from a discrete-time perspective in the existing literature [1,123]. Similarly, we examine the corresponding discrete-time system

$$
z(k+1)=Q(k) z(k)+\epsilon(k)
$$

where $z=\left[z_{1}, \ldots, z_{n}\right]^{\prime}, Q(k)$ is a doubly stochastic matrix, and $\epsilon(k)$ is the diminishing disturbance. Denote $\Delta z=(I-J) z$ and $\Delta \epsilon=(I-J) \epsilon$, where $J=I-\frac{1}{N} \mathbf{1}_{N} \mathbf{1}_{N}^{\prime}$. The following lemma validates the consensus of 6.16, whose proof can be referred to 36, Lemma 3.1]. 
Lemma 6.6. Let $\Gamma(k)$ be the graph associated with $Q(k)$ in 6.16 . Assume that the following holds:

1) There exists $T \in \mathcal{N}^{+}$such that $\Gamma[k, k+T)$ is strongly connected for each $k$;

2) $[Q(k)](i, i) \in[\bar{q}, 1], \forall i$, and $[Q(k)](i, j) \in\{0\} \cup[\bar{q}, 1], \forall i \neq j$, with constant $\bar{q} \in(0,1)$.

Then it holds that

$$
\|\Delta z(k)\| \leq \sqrt{2 N}\left[(1-\sigma)^{\frac{k}{N T-1}-1}\|\Delta z(0)\|+\sum_{n=0}^{k-1}(1-\sigma)^{\frac{k-1-n}{N T-1}-1}\|\Delta \epsilon(n)\|\right]
$$

where $\sigma=\bar{q}^{N(N+1) T / 2-1}$.

To apply the above result, some facts about the exponential of Laplacian matrices are needed.

Lemma 6.7. Let $L(t)$ be the Laplacian for the graph $\mathcal{G}(t)=\{\mathcal{N}, \mathcal{E}(t), W(t)\}$. Then the following holds for $\tau>0$

1) $Q(t ; \tau)=e^{-L(t) \tau}$ is doubly stochastic;

2) $[Q(t ; \tau)](i, i)>0$ for each $i$, and $[Q(t ; \tau)](i, j)>0$ iff there is a path from $j$ to $i$;

3) If $0<w_{*} \leq[W(t)](i, j) \leq w^{*}$ for $[W(t)](i, j)>0$, then there exists a constant $q^{*} \in(0,1)$ such that $[Q(t ; \tau)](i, j)>q^{*}$ for $[Q(t ; \tau)](i, j)>0$, as long as $\tau \in\left[\tau_{*}, \tau^{*}\right]$ with $\tau_{*}>0$.

Proof. 1) By the definition of the matrix exponential and $\mathbf{1}_{N}^{\prime} L(t)=L(t) \mathbf{1}_{N}=0$, it is readily seen that $Q(t ; \tau)$ is doubly stochastic.

2) By letting $a=(N-1) w^{*}+1$ and noticing that $Q(t ; \tau)=e^{\tau(-L(t)+a I)} e^{-a \tau I}$, we observe that $[Q(t ; \tau)](i, j)$ is nonnegative due to $-L(t)+a I>0$. Rewrite $Q(t ; \tau)$ as

$$
Q(t ; \tau)=\sum_{k=0}^{\infty} \frac{1}{k !} H^{k}(t) \tau^{k} e^{-a \tau I}
$$

where $H(t)=-L(t)+a I$ has positive diagonal entries. It is clear that the $(i, j)$-th entry of $H^{N-1}(t)$ and $H^{k}(t), k \geq N-1$ have the same $\operatorname{sign}(0$ or +$)$, because the length of a path in a graph of $n$ nodes is no more than $n-1$; moreover, the entry of $H^{N}(t)$ is positive iff there is a path between the corresponding vertexes, which confirms 2).

3) can be easily checked by the boundedness of $\tau$ and $[W(t)](i, j)$. 
Now we go back to consensus analysis. For any solution $X(t)=\operatorname{col}\left\{x_{1}(t), \cdots, x_{N}(t)\right\} \in \mathbb{R}^{m N}$, $\dot{X}(t)$ is locally integrable and it holds a.e. over $\left[t_{k}, t_{k+1}\right]$ that

$$
\dot{X}(t)=-\left[L\left(t_{k}\right) \otimes I_{m}\right] X(t)+\xi(t)
$$

where $\xi(t)=\operatorname{col}\left\{\xi_{1}(t), \cdots, \xi_{N}(t)\right\}$ with $\xi_{i}(t)=\alpha\left(t+\tau_{i}\right) s_{i}(t)+P \mathcal{X}\left(x_{i}(t)\right)-x_{i}(t)$, and $s_{i}(t) \in \partial f_{i}\left(x_{i}(t)\right)$. Clearly $\xi(t)$ is measurable as the difference of two measurable functions $\dot{X}(t)$ and $-\left[L\left(t_{k}\right) \otimes I_{m}\right] X(t)$. Consider the dynamics of the $l$-th coordinate $x_{i l}(t)$ described by

$$
\dot{x}^{(l)}(t)=L\left(t_{k}\right) x^{(l)}(t)+\xi^{(l)}(t), t \in\left[t_{k}, t_{k+1}\right] \text { a.e. },
$$

with $x^{(l)}(t)=\left[x_{1 l}(t), \ldots, x_{N l}(t)\right]^{\prime}$ and $\xi^{(l)}(t)$ comprised of the associated entries in $\xi(t)$. By Lemma 6.1 the solution to 6.18 is uniquely found as

$$
x^{(l)}(t)=e^{-L\left(t_{k}\right)\left(t-t_{k}\right)} x^{(l)}\left(t_{k}\right)+\int_{t_{k}}^{t} e^{-L\left(t_{k}\right)(t-\tau)} \xi^{(l)}(\tau) d \tau .
$$

Due to the global set convergence and the diminishing $\alpha(t)$, we may assume that $\left\|\xi^{(l)}(\tau)\right\| \leq \varepsilon$ when $\tau \geq t_{0}$ for any given $\varepsilon>0$. Noticing that $e^{-L\left(t_{k}\right)(t-\tau)}$ is doubly stochastic for $t \geq \tau$, we have $\left\|e^{-L\left(t_{k}\right)(t-\tau)}\right\| \leq 1$ by Lemma 3.3 and it turns out that

$$
\left\|\int_{t_{k}}^{t} e^{-L\left(t_{k}\right)(t-\tau)} \xi^{(l)}(\tau) d \tau\right\| \leq\left(t-t_{k}\right) \varepsilon .
$$

We further assume that $t_{k+1}-t_{k} \leq 2 \tau_{D}$, otherwise $\left\{T_{\kappa}\right\}_{\kappa=1}^{K}$ can be inserted between $t_{k}$ and $t_{k+1}$, with $T_{\kappa}=t_{k}+\kappa \frac{t_{k+1}-t_{k}}{K}$ and $(K+1) \tau_{D} \geq t_{k+1}-t_{k} \geq K \tau_{D}$. If $\mathcal{G}$ only switches at finite instants with the last switching instant $t_{e}$, we take the subsequent instants as $t_{e+\kappa}=t_{e}+\kappa \tau_{D}, \kappa \geq 1$. Now it is close to achieve the consensus result.

In fact, by Lemma 6.7, 6.20 and $\|I-J\|=1$ it is clear that

$$
\left\|\Delta x^{(l)}(t)\right\| \leq\left\|\Delta x^{(l)}\left(t_{k}\right)\right\|+2 \tau_{D} \varepsilon
$$

for $t \in\left[t_{k}, t_{k+1}\right]$, so it suffices to investigate $\Delta x^{(l)}\left(t_{k}\right)$. Letting $d t_{k}=t_{k+1}-t_{k}$, consider (6.16) with $z(k)=x^{(l)}\left(t_{k}\right), Q(k)=e^{-L\left(t_{k}\right) d t_{k}}$ and $\epsilon(k)=\int_{t_{k}}^{t_{k+1}} e^{-L\left(t_{k}\right)\left(t_{k+1}-\tau\right)} \xi^{(l)}(\tau) d \tau$. In the light of 6.20 and $t_{k+1}-t_{k} \leq 2 \tau_{D}$ we get $\|\Delta \epsilon(k)\| \leq 2 \tau_{D} \varepsilon$, and it remains to verify conditions 1) and 2) in Lemma 6.6. The condition 2) is already satisfied as a result of 3) in Lemma 6.7 if we remember Assumptions 6.3 and 6.4 . Besides, we know that $\mathcal{G}$ is balanced and uniformly contains joint spanning trees, which suggests the existence of a constant $T$ such that $\mathcal{G}\left(\left[t_{k}, t_{k+\left\lceil\frac{T}{\tau_{D}}\right\rceil+1}\right)\right)$ is strongly connected for every $k$. Now condition 1) is satisfied by observing that $\mathcal{E}\left(t_{k}\right) \subseteq \mathcal{E}(Q(k))$ by the result 2) of Lemma 6.7. In summary, 6.2a is achieved by letting $\varepsilon \rightarrow 0$. 


\subsubsection{Optimum Set Convergence}

In this subsection we shall show the optimum set convergence $6.2 \mathrm{~b}$ by contradiction. For sufficiently small $\varepsilon>0$, the convergence results 6.9 and $6.2 \mathrm{a}$ imply the existence of a time instant $t_{\varepsilon}$ such that $\forall t \geq t_{\varepsilon},|\bar{x}(t)|_{\mathcal{X}}+2 \max _{i}\left\|x_{i}(t)-\bar{x}(t)\right\| \leq \frac{\varepsilon}{N}$, where $\bar{x}(t)=\frac{1}{N} \sum_{i} x_{i}(t)$. By 6.10 it follows that

$$
\left|F\left(P_{\mathcal{X}}(\bar{x})\right)-F(\bar{x})\right|+\sum_{i}\left|f_{i}(\bar{x})-f_{i}\left(x_{i}\right)\right|+\sum_{i}\left|f_{i}\left(P_{\mathcal{X}^{*}}(\bar{x})\right)-f_{i}\left(P_{\mathcal{X}^{*}}\left(x_{i}\right)\right)\right| \leq \bar{\gamma} \varepsilon
$$

Furthermore by Remark 6.6, $t_{\varepsilon}$ can also be selected so that for some $M>0$

$$
\sum_{i}\left|\alpha\left(t+\tau_{i}\right)-\alpha(t) \| f_{i}\left(P_{\mathcal{X}^{*}}\left(x_{i}\right)\right)-f_{i}\left(x_{i}\right)\right| \leq M \varepsilon \alpha(t)
$$

Consider $l_{i}\left(x_{i}\right)=\left|x_{i}\right|_{\mathcal{X}^{*}}^{2}$ and $l(t)=\sum_{i} l_{i}\left(x_{i}(t)\right)$. Obviously the convergence to $\mathcal{X}^{*}$ is equivalent to the convergence of $l(t)$ to zero. By the definition of subdifferentials, it a.e. holds that

$$
\begin{aligned}
i_{i} \leq & 2 \sum_{j} w_{i j}(t)\left\langle x_{i}-P_{\mathcal{X}^{*}}\left(x_{i}\right), x_{j}-x_{i}\right\rangle \\
& +2 \alpha\left(t+\tau_{i}\right)\left(f_{i}\left(P_{\mathcal{X}^{*}}\left(x_{i}\right)\right)-f_{i}\left(x_{i}\right)\right) .
\end{aligned}
$$

Recalling Lemma 3.6 and $\sum_{j} w_{i j}(t)=\sum_{j} w_{j i}(t)$ for any $i$, we have

$$
\begin{aligned}
& 2 \sum_{i} \sum_{j} w_{i j}(t)\left\langle x_{i}(t)-P_{\mathcal{X}^{*}}\left(x_{i}\right), x_{j}-x_{i}\right\rangle \\
\leq & 2 \sum_{i} \sum_{j} w_{i j}(t)\left|x_{i}\right|_{\mathcal{X}^{*}}\left(\left|x_{j}\right|_{\mathcal{X}^{*}}-\left|x_{i}\right|_{\mathcal{X}^{*}}\right) \\
= & -\sum_{i} \sum_{j} w_{i j}(t)\left(\left|x_{j}\right|_{\mathcal{X}^{*}}-\left|x_{i}\right|_{\mathcal{X}^{*}}\right)^{2} \leq 0,
\end{aligned}
$$

which together with 6.21 and 6.22 yields that

$$
\begin{aligned}
i \leq & 2 \sum_{i} \alpha\left(t+\tau_{i}\right)\left(f_{i}\left(P_{\mathcal{X}^{*}}\left(x_{i}\right)\right)-f_{i}\left(x_{i}\right)\right) \\
= & 2 \alpha(t)\left[F\left(P_{\mathcal{X}^{*}}(\bar{x})\right)-F\left(P_{\mathcal{X}}(\bar{x})\right)+F\left(P_{\mathcal{X}}(\bar{x})\right)-F(\bar{x})\right. \\
& \left.-\sum_{i}\left(f_{i}\left(P_{\mathcal{X}^{*}}(\bar{x})\right)-f_{i}\left(P_{\mathcal{X}^{*}}\left(x_{i}\right)\right)\right)+\sum_{i}\left(f_{i}(\bar{x})-f_{i}\left(x_{i}\right)\right)\right] \\
& +2 \sum_{i}\left(\alpha\left(t+\tau_{i}\right)-\alpha(t)\right)\left[f_{i}\left(P_{\mathcal{X}^{*}}\left(x_{i}\right)\right)-f_{i}\left(x_{i}\right)\right] \\
\leq & 2 \alpha(t)\left[F\left(P_{\mathcal{X}^{*}}(\bar{x})\right)-F\left(P_{\mathcal{X}}(\bar{x})\right)+(M+\bar{\gamma}) \varepsilon\right], t \geq t_{\varepsilon} .
\end{aligned}
$$

Next we shall show that $\lim _{t \rightarrow \infty} l(t)=0$ by making use of 6.24 . 
1) $\liminf _{t \rightarrow \infty} l(t)=0$. We first show that $\liminf _{t \rightarrow \infty}\left\|P_{\mathcal{X}}(\bar{x})-P_{\mathcal{X}}(\bar{x})\right\|=0$. Otherwise there exists $t_{1}$ such that $\left\|P_{\mathcal{X}}(\bar{x}(t))-P_{\mathcal{X}^{*}}(\bar{x}(t))\right\| \geq \delta_{1}>0$ when $t \geq t_{1}$ and consequently $F\left(P_{\mathcal{X}}(\bar{x}(t))\right)-$ $F\left(P_{\mathcal{X}^{*}}(\bar{x}(t))\right) \geq \delta_{2}>0$, if we recall that $P_{\mathcal{X}^{*}}(\bar{x}) \in \mathcal{X}^{*}$ and $F$ is convex and coercive. Letting $\varepsilon=\frac{\delta_{2}}{2(\bar{\gamma}+M)}$ and substituting it into 6.24, it holds a.e. that

$$
i(t) \leq 2 \alpha(t)\left(-\delta_{2}+\delta_{2} / 2\right)=-\alpha(t) \delta_{2}, t \geq t_{2} \triangleq \max \left(t_{1}, t_{\varepsilon}\right) .
$$

Integrating on both sides gives $l(t) \leq l\left(t_{2}\right)-\delta_{2} \int_{t_{2}}^{t} \alpha(t) d t$, suggesting that $l(t)$ will become negative when $t$ is sufficiently large by the persistence condition 6.5, which is a contradiction. Hence $\liminf _{t \rightarrow \infty}\left\|P_{\mathcal{X}}(\bar{x}(t))-P_{\mathcal{X}^{*}}(\bar{x}(t))\right\|=0$. Moreover, by the constraint set convergence and consensus result we obtain $\liminf _{t \rightarrow \infty} l(t)=0$.

2) $\limsup l(t)=0$. Assume this is not the case and $\limsup l(t)=\delta_{3}>0$. Noticing that $\liminf _{t \rightarrow \infty} l(t)=0$, there must be an infinite sequence of local maximizers $\left\{t_{n_{k}}\right\}$ with $\delta_{3}=\limsup _{k \rightarrow \infty} l\left(t_{n_{k}}\right)$. However, $D^{-} l\left(t_{n_{k}}\right) \geq 0$ at the local maximizer, while by noting (6.24) and the derivation of $(6.12)$ we have

$$
D^{-} l\left(t_{n_{k}}\right) \leq 2 \alpha(t)\left[F\left(P_{\mathcal{X}^{*}}\left(\bar{x}\left(t_{n_{k}}\right)\right)\right)-F\left(P_{\mathcal{X}}\left(\bar{x}\left(t_{n_{k}}\right)\right)\right)+\bar{\gamma} \varepsilon\right]
$$

which implies that $F\left(P_{\mathcal{X}}\left(\bar{x}\left(t_{n_{k}}\right)\right)\right)-F\left(P_{\mathcal{X}^{*}}\left(\bar{x}\left(t_{n_{k}}\right)\right)\right) \leq \bar{\gamma} \varepsilon$ when $t_{n_{k}} \geq t_{\varepsilon}$. By the arbitrariness of $\varepsilon$ it can be seen that $P_{\mathcal{X}}\left(\bar{x}\left(t_{n_{k}}\right)\right) \rightarrow P_{\mathcal{X}^{*}}(\bar{x})$ and $\lim \sup l\left(t_{n_{k}}\right)=0$, which is a contradiction. Hence $\limsup _{t \rightarrow \infty} l(t)=0$ and $6.2 \mathrm{~b}$ holds.

Remark 6.8. Problem (6.1) is still tractable if $f_{i}$ is only convex, with a compact and convex $\mathcal{X}$. All the analysis can proceed similarly except that of the global boundedness of solution. Noting that $\left\langle\partial f_{i}\left(x_{i}\right), x_{i}-x^{*}\right\rangle>0$ does not necessarily hold even when $x_{i}$ is far away from $x^{*}$, the boundedness of the solution is achieved by combining the monotonicity property of subdifferentials, and the boundedness of $\mathcal{X}$. Indeed, the monotonicity property 103] dictates that $\left\langle s, x_{i}-y^{*}\right\rangle \geq\left\langle r_{i}, x_{i}-y^{*}\right\rangle, \forall s \in \partial f_{i}\left(x_{i}\right)$, $r_{i} \in \partial f_{i}\left(y^{*}\right)$. Consequently we derive

$$
D^{+} d(t) \leq 2\left[-d(t)+\max _{i} \alpha\left(t+\tau_{i}\right) M \sqrt{d(t)}\right]
$$

where $M=\max _{i} \max _{r_{i} \in \partial f_{i}\left(y^{*}\right)}\left\|r_{i}\right\|$. The boundedness of the solution is attained by applying the comparison principle.

\subsection{Convergence Rate Analysis}

In this section we aim to analyze the convergence rate of the algorithm. Corresponding to Subsections 6.3 .3 - 6.3 .5 the convergence rate analysis consists of 3 parts: set convergence rate, consensus rate, 
and optimum set convergence rate. Note that the rate analysis in the first two parts does not need additional conditions other than those given in Theorem 6.1.

\subsubsection{Set Convergence Rate}

Consider $y=\frac{p(t)}{\alpha^{2}(t)}$ where $p(t)$ has been defined in Section 6.3.3. Similar to the derivation of 6.13 we get

$$
\begin{aligned}
D^{+} y & \leq-2 \frac{p(t)}{\alpha^{2}(t)}+2 \bar{\gamma} \frac{\max _{i} \alpha\left(t+\tau_{i}\right) \sqrt{p(t)}}{\alpha^{2}(t)}-2 \frac{\dot{\alpha}(t)}{\alpha(t)} \frac{p(t)}{\alpha^{2}(t)} \\
& \leq-2 \frac{p(t)}{\alpha^{2}(t)}+2 \bar{\gamma} e^{\varepsilon(t) \bar{\tau}} \frac{\sqrt{p(t)}}{\alpha(t)}+2 \varepsilon(t) \frac{p(t)}{\alpha^{2}(t)} \\
& =-2[1-\varepsilon(t)] y+2 \bar{\gamma} e^{\varepsilon(t) \bar{\tau}} \sqrt{y}
\end{aligned}
$$

where $\varepsilon(t) \rightarrow 0$ as $t \rightarrow \infty$ in view of Remark 6.6. Also note that we can assume $y(t)>0$ for $t \geq t_{0}$. Therefore, we obtain from 6.25 that

$$
D^{+} \sqrt{y} \leq-(1-\varepsilon(t)) \sqrt{y}+\bar{\gamma} e^{\varepsilon(t) \tau}
$$

which follows that $\limsup _{t \rightarrow \infty} \sqrt{y} \leq \bar{\gamma}$. As a result, we get

$$
\max _{i}\left|x_{i}(t)\right|_{\mathcal{X}}=O(\alpha(t))
$$

\subsubsection{Consensus Rate}

Here we use the same notations as in Section 6.3.4. From the bounded subgradient result 6.10, set convergence result 6.27), and Remark 6.6, we know that there exists a constant $C_{1}>0$ such that

$$
\left\|\xi_{i}(t)\right\|=\left\|\alpha\left(t+\tau_{i}\right) s_{i}(t)+P_{\mathcal{X}}\left(x_{i}(t)\right)-x_{i}(t)\right\| \leq C_{1} \alpha\left(t_{k}\right), t \in\left[t_{k+1}, t_{k}\right] .
$$

Then it holds that $\left\|\int_{t_{k}}^{t} e^{-L\left(t_{k}\right)(t-\tau)} \xi^{(l)}(\tau) d \tau\right\| \leq C_{1}\left(t-t_{k}\right) \alpha\left(t_{k}\right)$ for $t \in\left[t_{k_{+1}}, t_{k}\right]$, and by applying Lemma 6.6 there exist constants $C>0$ and $\rho \in(0,1)$ such that

$$
\left\|\Delta x^{(l)}\left(t_{k}\right)\right\| \leq C \rho^{k}\left\|\Delta x^{(l)}\left(t_{0}\right)\right\|+C \sum_{n=0}^{k-1} \rho^{k-1-n} \alpha\left(t_{n}\right)
$$

Now consider $y^{(l)}(t)=\frac{\left\|\Delta x^{(l)}(t)\right\|}{\alpha(t)}$. Noticing that $\lim _{t \rightarrow \infty} \frac{\alpha\left(t_{k}\right)}{\alpha\left(t_{k+1}\right)}=1$ follows as a result of Assumption 6.6 and $t_{k+1}-t_{k} \leq 2 \tau_{D}$, we can find a constant $\tilde{\rho} \in(0,1)$ such that $\rho \lim _{k \rightarrow \infty} \frac{\alpha\left(t_{k}\right)}{\alpha\left(t_{k+1}\right)}<\tilde{\rho}<1$. Without loss 
of generality we assume that $\rho \frac{\alpha\left(t_{k}\right)}{\alpha\left(t_{k+1}\right)}<\tilde{\rho}$ for all $k \geq 0$. Therefore, by 6.28 we obtain that

$$
\begin{aligned}
y^{(l)}\left(t_{k}\right) & \leq C \frac{\rho^{k}}{\alpha\left(t_{k}\right)}\left\|\Delta x\left(t_{0}\right)\right\|+C \sum_{n=0}^{k-1} \rho^{k-1-n} \frac{\alpha\left(t_{n}\right)}{\alpha\left(t_{k}\right)} \\
& =C \rho^{k} y^{(l)}\left(t_{0}\right) \prod_{n=0}^{k-1} \frac{\alpha\left(t_{n}\right)}{\alpha\left(t_{n+1}\right)}+\frac{C}{\rho} \sum_{n=0}^{k-1} \rho^{k-n} \prod_{s=0}^{k-n} \frac{\alpha\left(t_{s}\right)}{\alpha\left(t_{s+1}\right)} \\
& \leq C \tilde{\rho}^{k} y^{(l)}\left(t_{0}\right)+\frac{C}{\rho} \sum_{n=0}^{k-1} \tilde{\rho}^{k-n} \leq C\left[y^{(l)}\left(t_{0}\right)+\frac{\tilde{\rho}}{\rho(1-\tilde{\rho})}\right] .
\end{aligned}
$$

As for $t \in\left(t_{k}, t_{k+1}\right)$, we can see from 6.19 that

$$
y^{(l)}(t) \leq \frac{\alpha\left(t_{k}\right)}{\alpha(t)}\left[y^{(l)}\left(t_{k}\right)+\left(t-t_{k}\right)\right]
$$

which implies that $\limsup _{t \rightarrow \infty} y^{(l)}(t) \leq \infty$. Hence, we have found the consensus rate as

$$
\left\|x_{i}(t)-\bar{x}(t)\right\|=O(\alpha(t))
$$

\subsubsection{Optimum Set Convergence Rate}

In this section we shall study the rate of optimum set convergence for different types of $\alpha(t)$, provided that the aggregate cost $F=\sum_{i} f_{i}$ is $\beta$-strongly convex over $\mathcal{X}$. We present our main result as follows.

Theorem 6.2. Assume that the conditions in Theorem 6.1 are satisfied, except that the condition 2) is replaced by

$$
\limsup _{t \rightarrow \infty}|r(t)|<\infty, r(t)=\frac{\dot{\alpha}(t)}{\alpha^{2}(t)} .
$$

Moreover, assume the aggregate cost $F=\sum_{i} f_{i}$ is $\beta$-strongly convex over $\mathcal{X}$, and denote the unique minimum of $F$ over $\mathcal{X}$ as $x^{*}$. Then we have the following estimation about the convergence rate to $x^{*}:$

1) If $\liminf _{t \rightarrow \infty} r(t)>-\frac{\beta}{N}$, then $\left\|x_{i}(t)-x^{*}\right\|^{2}=O(\alpha(t))$;

2) If $r(t)=-\frac{\beta}{N}+O(\alpha(t))$, then $\left\|x_{i}(t)-x^{*}\right\|^{2}=O\left(\alpha(t) \int_{t_{0}}^{t} \alpha(\tau) d \tau\right)$;

3) If $-p<\liminf _{t \rightarrow \infty} r(t) \leq-\frac{\beta}{N}$ and $\limsup _{t \rightarrow \infty} r(t)<0$, then $\left\|x_{i}(t)-x^{*}\right\|^{2}=O\left(\alpha^{\frac{\beta}{p N}}(t)\right)$.

Remark 6.9. Theorem 6.2 reveals an interesting relationship between the choice of decaying subgradient gain and the convergence rate, which is also closely related with the structure of the cost function and the number of agents. Roughly speaking, the 3 cases discussed in Theorem 6.2 correspond to the following 3 types of $\alpha(t): \alpha(t)>\frac{N}{\beta t}, \alpha(t)=\frac{N}{\beta t}$ and $\alpha(t)<\frac{N}{\beta t}$. Note that gains of the form $\frac{c}{t^{\gamma}}, 0<\gamma \leq 1, c>0$ are covered by these cases. 
Remark 6.10. We can see in the proof that any step size satisfying condition (6.31) is lower bounded by $O\left(\frac{1}{t}\right)$. From Theorem 6.2 we observe that the convergence rate is at most of the same order of $\alpha(t)$, and hence we need to select a smaller $\alpha(t)$ for a faster rate. Therefore, the fastest convergence rate can be achieved by selecting step sizes from $\alpha(t)=\frac{c}{t}$, and it is readily seen that the best choice is $\alpha(t)=\frac{c}{t}$ with $c>\frac{N}{\beta}$. In this case, $x_{i}(t)$ converges to the optimum $x^{*}$ at the rate of $O\left(\frac{1}{\sqrt{t}}\right)$.

Before proceeding to the proof, let us introduce the following lemma.

Lemma 6.8. Assume that a function $y:\left[t_{0}, \infty\right) \rightarrow \mathbb{R}$ satisfies $D y \leq c(t)\left(c_{1}-c_{2} y\right)$, where $D$ denotes a certain kind of Dini derivative. If $c_{1}, c_{2}>0$, and $c(t)>0$ is a continuous function with $\int_{t_{0}}^{\infty} c(t) d t=\infty$, then $\limsup _{t \rightarrow \infty} y(t) \leq \frac{c_{1}}{c_{2}}$.

Proof. We may assume that $y$ satisfies $\dot{y}=c(t)\left(c_{1}-c_{2} y\right)$, otherwise we impose the comparison principle. Let $z=e^{c_{2} \int_{t_{0}}^{t} c(\tau) d \tau} y$. Then

$$
\begin{aligned}
\dot{z} & =e^{c_{2} \int_{t_{0}}^{t} c(\tau) d \tau}\left(\dot{y}+c_{2} y\right) \\
& =c_{1} c(t) e^{c_{2} \int_{t_{0}}^{t} c(\tau) d \tau} \\
& =\frac{c_{1}}{c_{2}} \frac{d}{d t}\left(e^{c_{2} \int_{t_{0}}^{t} c(\tau) d \tau}+\tilde{c}\right),
\end{aligned}
$$

where $\tilde{c}$ is a constant. Therefore, $y=\frac{c_{1}}{c_{2}}\left(1+\tilde{c} e^{-c_{2} \int_{t_{0}}^{t} c(\tau) d \tau}\right)$, and the result follows by noticing that $\int_{t_{0}}^{\infty} c(t) d t=\infty$.

Proof of Theorem 6.2. First of all, we show the optimal consensus when Assumptions 6.5 and 6.6 are replaced by 6.31. Actually, now we can find a constant $p_{1}>0$ such that $r(t)>-p_{1}$ for sufficiently large $t$, which leads to $O\left(\frac{1}{p_{1} t}\right) \leq \alpha(t)$ and Assumption 6.5 can be satisfied. Moreover, it is clear to see that Assumption 6.6 holds with the decaying $\alpha(t)$ and 6.31. In consequence, the conditions of Theorem 6.1 are all satisfied and the convergence to $\mathcal{X}$ is established.

The proof of convergence rate is predicated on a further analysis of (6.24). First of all, the condition 6.31 dictates that

$$
|\alpha(t+\tau)-\alpha(t)|=O\left(\alpha^{2}(t)\right), \forall \tau \in[0, \bar{\tau}]
$$

Actually, we know by the mean value theorem that there exists some $\xi \in(0, \tau)$ such that

$$
\frac{\alpha(t+\tau)-\alpha(t)}{\alpha^{2}(t)}=\tau \frac{\dot{\alpha}(t+\xi)}{\alpha^{2}(t)}=\tau \frac{\dot{\alpha}(t+\xi)}{\alpha^{2}(t+\xi)} \frac{\alpha^{2}(t+\xi)}{\alpha^{2}(t)}
$$


and the result is achieved in the light of Remark 6.6. Secondly, from the set convergence 6.27), consensus 6.30 and the global boundedness of $x_{i}$ we observe that

$$
\begin{aligned}
\left\|x_{i}(t)-x^{*}\right\|^{2} & =\left\|x_{i}(t)-\bar{x}(t)+\bar{x}(t)-P_{\mathcal{X}}(\bar{x}(t))+P_{\mathcal{X}}(\bar{x}(t))-x^{*}\right\|^{2} \\
& \leq\left\|P_{\mathcal{X}}(\bar{x}(t))-x^{*}\right\|^{2}+O(\alpha(t)) .
\end{aligned}
$$

Imposing 6.24 and noticing that $\mathcal{X}=\left\{x^{*}\right\}$, we obtain that

$$
i \leq 2 \alpha(t)\left[F\left(x^{*}\right)-F\left(P_{\mathcal{X}}(\bar{x})\right)+F\left(P_{\mathcal{X}}(\bar{x})\right)-F(\bar{x})+\sum_{i}\left(f_{i}(\bar{x})-f_{i}\left(x_{i}\right)\right)\right]+2 O\left(\alpha^{2}(t)\right) .
$$

Now combine 6.32, 6.33) and Lemma 3.9 about the $\beta$-strongly convex function $F$, and we get that $i \leq \alpha(t)\left(-\frac{\beta}{N}+O(\alpha(t))\right)$. Therefore, we can find a constant $M>0$ such that

$$
i \leq \alpha(t)\left(-\frac{\beta}{N}+M \alpha(t)\right)
$$

Let $y=\frac{l}{\alpha \eta}$, then it can be verified that

$$
\dot{y} \leq-\left(\frac{\beta}{N}+\frac{\dot{\alpha}}{\alpha}+\frac{\dot{\eta}}{\eta}\right) y+M \frac{\alpha}{\eta}
$$

Note that the above inequality actually holds a.e. for $y$ as 6.24 holds a.e., and by applying Lemma 6.3 we can find that

$$
D y \leq-\left(\frac{\beta}{N}+\frac{\dot{\alpha}}{\alpha}+\frac{\dot{\eta}}{\eta}\right) y+M \frac{\alpha}{\eta},
$$

where $D$ denotes a certain type of Dini derivative.

1) Noticing that $-\frac{\beta}{N}<\liminf _{t \rightarrow \infty} r(t)$, we can find $b>0$ such that $r(t)>-b>-\frac{\beta}{N}$. Moreover, by letting $\eta=1$ in 6.36, we can obtain from 6.36 that

$$
D y \leq \alpha(t)\left[M-\left(\frac{\beta}{N}-b\right) y\right] .
$$

Now apply Lemma 6.8 and we get $\limsup _{t \rightarrow \infty} y(t)<\infty$, or $\left\|x_{i}(t)-x^{*}\right\|^{2}=O(\alpha(t))$.

2) Let $\eta=\int_{t_{0}}^{t} \alpha(\tau) d \tau$, then we have $\frac{\dot{\eta}}{\eta}=\frac{\alpha(t)}{\int_{t_{0}}^{t} \alpha(\tau) d \tau}$. Moreover, by the condition $r(t)=-\frac{\beta}{N}+$ $O(\alpha(t))$, we can obtain from (6.36) that

$$
D y \leq \frac{\alpha(t)}{\eta(t)}[M-(1-O(\alpha \eta)) y] .
$$

Noticing that $\frac{\alpha(t)}{\eta(t)}=\frac{d}{d t} \ln \int_{t_{0}}^{t} \alpha(\tau) d \tau$, we have $\int_{t_{1}}^{\infty} \frac{\alpha(t)}{\eta(t)} d t=\infty$, where $t_{1}>t_{0}$. On the other hand, by L'Hospital's Rule it is readily seen that $\lim _{t \rightarrow \infty} \alpha \eta=-\lim _{t \rightarrow \infty} \frac{\alpha^{3}}{\dot{\alpha}}=0$. Therefore, similar to 1) we apply Lemma 6.8 to 6.36p to obtain $\left\|x_{i}(t)-x^{*}\right\|^{2}=O\left(\alpha(t) \int_{t_{0}}^{t} \alpha(\tau) d \tau\right)$. 
3) By the given condition we can see that there exists a constant $p_{1}>0$ such that $-p<r(t)<$ $-p_{1}$ holds for sufficient large $t$, which implies that

$$
O\left(\frac{1}{p t}\right) \leq \alpha(t) \leq O\left(\frac{1}{p_{1} t}\right)
$$

Denote $c=\frac{\beta}{p N}$ and let $\eta=\alpha^{c-1}$. Then it can be checked by 6.36 that

$$
D y \leq M \alpha^{2-c}(t)
$$

Now it can be seen from 6.37 that $\int_{t_{0}}^{\infty} \alpha^{2-c}(t) d t<\infty$, since $c<1$. Hence $\limsup y(t)<\infty$, which means that $\left\|x_{i}(t)-x^{*}\right\|^{2}=O\left(\alpha^{\frac{\beta}{p N}}(t)\right)$.

\subsection{Numerical Examples}

In this section, we illustrate our result by a numerical example mentioned in Remark 6.1, i.e. the calculation of the geometric median under a set constraint. Consider 4 agents with initial positions given by $x_{1}(0)=[0,0]^{\prime}, x_{2}(0)=[2,-1]^{\prime}, x_{3}(0)=[3,3]^{\prime}, x_{4}(0)=[1,4]^{\prime}$, and each agent only knows its own initial position. The communication graph $\mathcal{G}$ alternates between $\mathcal{G}_{1}$ and $\mathcal{G}_{2}$ according to the following rule

$$
\mathcal{G}(t)= \begin{cases}\mathcal{G}_{1}, & t \in \bigcup_{k=1}^{\infty}[2 k, 2 k+1), \\ \mathcal{G}_{2}, & \text { else }\end{cases}
$$

where $\mathcal{G}_{1}$ and $\mathcal{G}_{2}$ are shown in Fig. 6.1 with 0-1 edge weights. Obviously, $\mathcal{G}$ stays balanced and

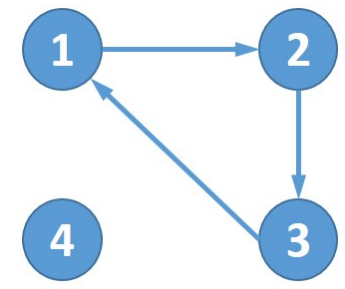

(a) $\mathcal{G}_{1}$

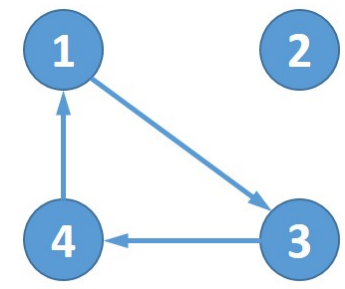

(b) $\mathcal{G}_{2}$

Figure 6.1: Communication graphs.

uniformly contains joint spanning trees, and the edge weights satisfy Assumption 6.4. The cost function for each agent is defined as $f_{i}\left(x_{i}\right)=\left\|x_{i}-x_{i}(0)\right\|$, and the time-varying gain is chosen as $\alpha(t)=\frac{1}{1+t^{0.5} \ln (t+1)}$ which is neither integrable nor square integrable. Note that the $\partial f_{i}$ is found as

$$
\partial f_{i}(x)= \begin{cases}\bar{B}(0,1), & x=x_{i}(0) \\ \frac{x-x_{i}(0)}{\left\|x-x_{i}(0)\right\|}, & \text { otherwise }\end{cases}
$$


where $\bar{B}(0,1)$ is the closed unit disk centered at the origin.

First, we consider the optimization problem without constraint, i.e. $\mathcal{X}=\mathbb{R}^{2}$. In this case, all the agents converge to the global optimum $[1.5,1.5]^{\prime}$ as shown in Fig. 6.3, which is the crossing point of the diagonals of the quadrilateral with $x_{i}(0)$ 's as its corners. The convergence of $x$-coordinates and

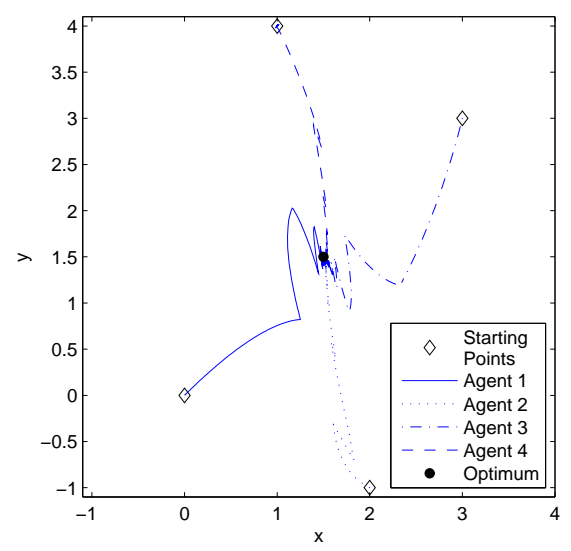

Figure 6.2: Continuous-time optimal consensus without set constraint.

$y$-coordinates of the four agents is shown in Fig. 6.3. Next, we incorporate the constraint set of the
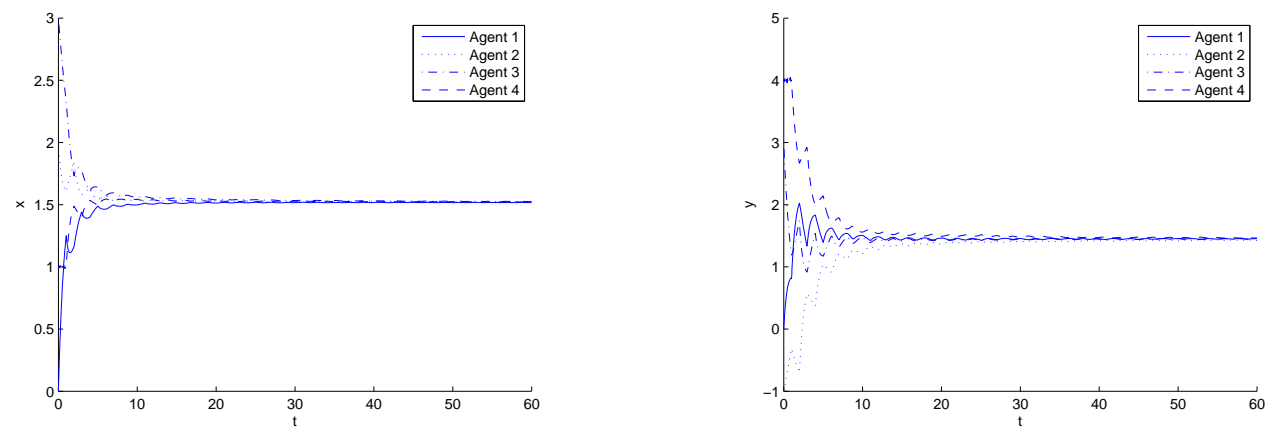

Figure 6.3: Convergence of $x, y$ coordinates without set constraint.

closed unit disk centered at origin, i.e. $\mathcal{X}=\bar{B}(0,1)$. From Fig. 6.4 we can see that the agents converge to the optimum $[0.8152,0.5792]^{\prime}$ on the unit circle. The convergence of $x$ and $y$ coordinates of the four agents is shown in Fig. 6.5.

\subsection{Conclusion}

In this chapter we considered the distributed constrained optimal consensus problem for continuoustime MASs. Each agent is assigned with a local objective function and a common set constraint. The control input of each agent is comprised of three parts: local information averaging, local projection, 


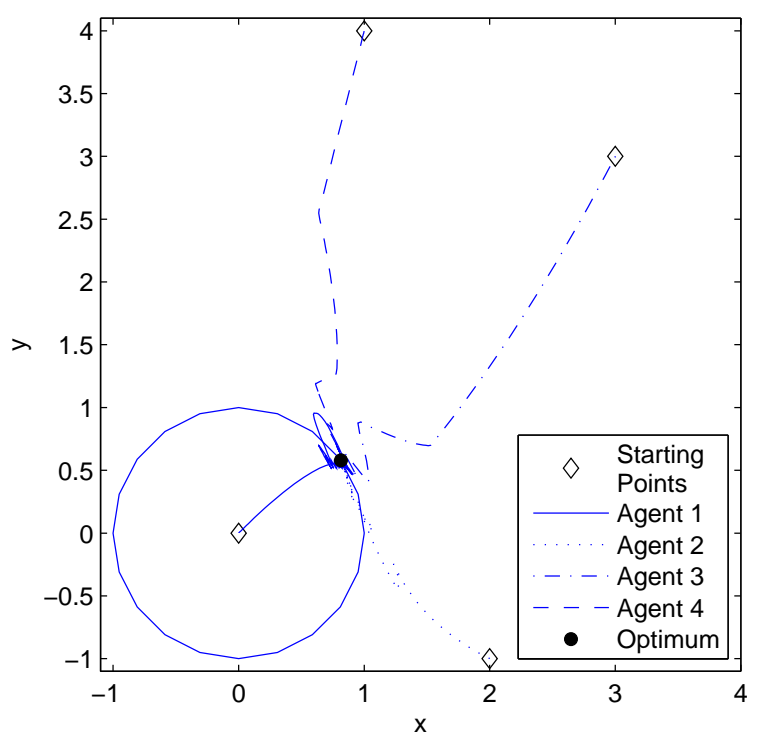

Figure 6.4: Continuous-time optimal consensus with $\mathcal{X}=\{x:\|x\| \leq 1\}$.
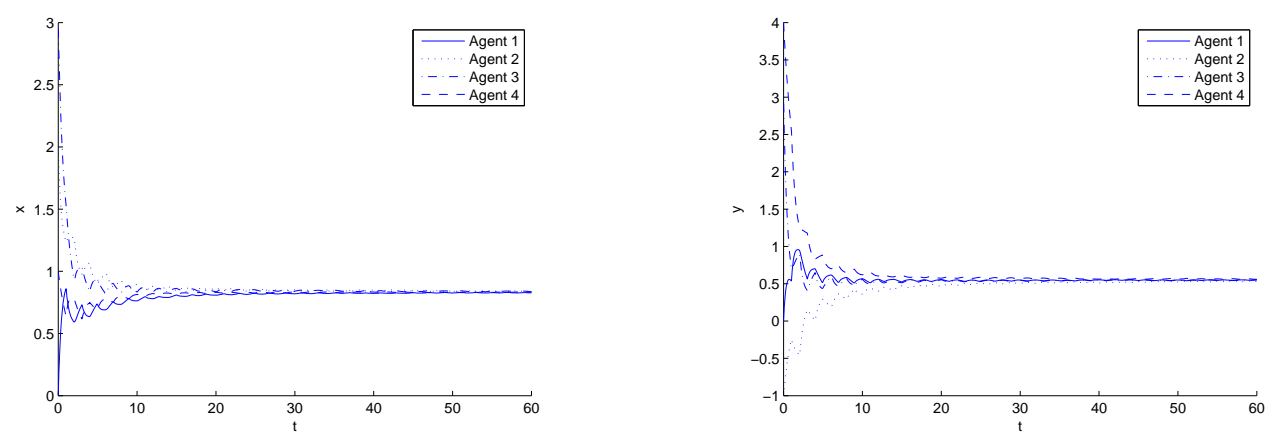

Figure 6.5: Convergence of $x, y$ coordinates with $\mathcal{X}=\{x:\|x\| \leq 1\}$.

and local subgradient. We investigated the problem under a balanced network with joint spanning trees. Under a persistence condition of subgradient gain and a standard assumption about varying edge weights, it was shown that all the states asymptotically converge to the optimal consensus point within the constraint set. Given a strongly convex cost, a systematic convergence rate analysis was also conducted for different types of subgradient gains, and it was suggested that the convergence rate is not only dependent on the subgradient gain, but also closely related with the cost function and the number of agents. 


\section{Chapter 7}

\section{Discrete-time Optimal Consensus}

\section{under Set Constraint}

In this chapter, we study a distributed optimal consensus problem of discrete-time MASs under a common set constraint. As with the continuous-time case, each agent is required to reach the minimum of the aggregate cost within the given constraint by exchanging information with neighbors, but now only part of agents is able to access the constraint set from time to time. We design a two-step control protocol: each agent firstly gets an intermediate state as a weighted average of its own state and those of its neighbors, and then it moves a local subgradient descent step, as well as a step along the projection vector when the constraint set is accessible. Given that at least one agent can frequently access the constraint set, we achieve the optimal consensus within the set constraint if the communication network stays balanced and uniformly contains joint spanning trees, and the time-varying step size of the subgradient descent satisfies a persistence condition. We also provide a systematic analysis of convergence rate for different step sizes, when the aggregate cost is strongly convex. For a fixed network, we further discuss necessary conditions to ensure the convergence, as well as the convergence result in the presence of fixed communication delays.

The rest of this chapter is organized as follows. We first formulate the problem and design a control protocol in Section 7.1, and then provide the convergence result and the corresponding analysis in Section 7.2 Next, we analyze the convergence rate for strongly convex cost functions in Section 7.3 Section 7.4 is dedicated to the discussion of necessary conditions and the issue of fixed time delay under a fixed communication network. Numerical examples are provided in Section 7.5, and we conclude the chapter in Section 7.6 . 


\subsection{Problem Formulation and Control Protocol}

Consider $N$ discrete-time agents with the first-order integrator dynamics as

$$
x_{i}(k+1)=x_{i}(k)+u_{i}(k), i=1, \ldots, N,
$$

where $x_{i}(k) \in \mathbb{R}^{m}$ and $u_{i}(k) \in \mathbb{R}^{m}$ are respectively the state and control input of agent $i$ at time step $k$. Besides, agent $i$ is assigned with a cost function $f_{i}$. As with the last chapter, the whole group is required to reach consensus within a given constraint set $\mathcal{X}$ (which is only accessible to part of agents), with the final consensus value minimizing the aggregate cost as a sum of $f_{i}$ 's. In summary, it is equivalent to solving the following problem:

$$
\min _{x_{i} \in \mathcal{X}} \sum_{i} f_{i}\left(x_{i}\right) \text {, s.t. } x_{1}=\cdots=x_{N} \text {. }
$$

The following assumptions are respectively about the constraint set and cost functions.

Assumption 7.1. $\mathcal{X}$ is closed and convex.

Assumption 7.2. For each $i, f_{i}: \mathbb{R}^{m} \rightarrow \mathbb{R}$ is convex and coercive.

Under Assumptions 7.1 and 7.2 the optimum set of $F=\sum_{i} f_{i}(x)$ within $\mathcal{X}$ must be convex and compact, and we denote it as $\mathcal{X}^{*}$. Therefore, 7.2 is solved asymptotically if we can design a distributed control protocol such that

$$
\begin{gathered}
\lim _{k \rightarrow \infty}\left(x_{i}(k)-x_{j}(k)\right)=0, i, j=1, \ldots, N, \\
\lim _{k \rightarrow \infty}\left|x_{i}(k)\right|_{\mathcal{X}^{*}}=0, i=1, \ldots, N .
\end{gathered}
$$

In a similar vein to the continuous-time case, the distributed control protocol is designed as below:

$$
\begin{aligned}
s_{i}(k) & =x_{i}(k)+\eta \sum_{j \in \mathcal{N}_{i}} w_{i j}(k)\left(x_{j}(k)-x_{i}(k)\right), \\
x_{i}(k+1) & =s_{i}(k)+h\left[b_{i}(k)\left(P_{\mathcal{X}}\left(s_{i}(k)\right)-s_{i}(k)\right)-\alpha(k) g_{i}\left(s_{i}(k)\right)\right],
\end{aligned}
$$

where $s_{i}(k)$ is an intermediate state and $g_{i}\left(s_{i}(k)\right) \in \partial f_{i}\left(s_{i}(k)\right) . w_{i j}(k)$ is the weight on communication edge $(j, i) . \quad b_{i}(k)=b>0$ if the agent $i$ can access $\mathcal{X}$, otherwise $b_{i}(k)=0$. Note that the proposed algorithm (7.4) consists of two steps: the first step is an averaging step trying to achieve consensus; while in the second step, each agent moves along the projection direction if $\mathcal{X}$ is accessible, together with a decaying step of subgradient descent, as an effort for the constrained optimization. 
Remark 7.1. The distributed control law (7.4) is similar to (6.3) by combining subgradient descent, projection, and local averaging. However, here we consider a more practical scenario where the constraint set can only be accessible by part of agents, which introduces the extra $b_{i}(k)$ into the control input. Hence, the step size b of the movement along the projection has to be designed. Noticing that the synchronization can be always achieved in the discrete-time case, we simply assume the time-varying gain $\alpha(k)$ to be synchronized.

\subsection{Convergence Analysis}

In this section, we shall first present the main result under specific assumptions and then analyze the convergence of 7.4 ).

Theorem 7.1. Suppose Assumptions 7.1 and 7.2 hold. Given that $\max _{i}\left\|x_{i}(0)-z\right\| \leq R$ for some $z \in \mathcal{X}$, there exist positive constants $\bar{\eta}$ and $\bar{h}$ such that the constrained optimal consensus problem 7.3 can be solved by the algorithm (7.4) with $\eta \in(0, \bar{\eta}), h \in(0, \bar{h})$ and $b \in(0,1)$, if the following conditions are satisfied:

1) The communication graph $\mathcal{G}$ stays balanced and uniformly contains joint spanning trees, and $0<w_{*} \leq w_{i j}(k) \leq w^{*}$ whenever $w_{i j}(k)>0 ;$

2) There exists a constant $T \in \mathbb{N}^{+}$such that at least one agent can access $\mathcal{X}$ in every $T$ time steps;

3) $\alpha(k)$ is diminishing i.e. $\lim _{k \rightarrow \infty} \alpha(k)=0$, and satisfies the persistence condition

$$
\sum_{k=0}^{\infty} \alpha(k)=+\infty
$$

Remark 7.2. The choices of $\bar{\eta}$ and $\bar{h}$ are specified as follows.

1) $\bar{\eta}=1 / \bar{D}$, where $\bar{D}=\max _{k \geq 0} \bar{D}(k)$ is the maximum degree of all graphs $\mathcal{G}(k)$.

2) To determine $\bar{h}$, first select a sufficiently large $R$ such that $\max _{i}\left\|x_{i, 0}-z\right\| \leq R$ and

$$
\left|f_{i}(x)-f_{i}(z)\right| \geq \delta>0,\|x-z\| \geq R
$$

Note that the above is feasible in light of the coerciveness of each $f_{i}$. Furthermore, denote

$$
\begin{aligned}
& \bar{R}=R+2 \bar{\alpha} \max _{i,\|x-z\| \leq R} \max _{g_{i}(x) \in \partial f_{i}(x)}\left\|g_{i}(x)\right\|, \\
& \bar{\gamma}=\max _{i,\|x-z\| \leq \bar{R}} \max _{g_{i}(x) \in \partial f_{i}(x)}\left\|g_{i}(x)\right\|,
\end{aligned}
$$


where $\bar{\alpha}=\max _{k} \alpha(k) \geq 1$. Now $\bar{h}$ can be selected as $\bar{h}=\min \left\{\frac{2 \delta}{\gamma^{2}+2 \bar{\gamma} \bar{R}}, 1\right\}$. In some cases, $\bar{h}$ can be a constant independent of the initial values, as revealed in the numerical example in Section 7.5

Proof. To show the convergence, we shall show in sequence the global boundedness of the system, constraint set convergence, consensus, and optimum set convergence.

1) Global boundedness of the system. We need to prove that $\max _{i}\left\|x_{i}(k)-z\right\| \leq \bar{R}$, where $\bar{R}$ is defined in (7.7). Without loss of generality, we assume that $z=0 \in \mathcal{X}$.

Obviously $\max _{i}\left\|x_{i}(0)\right\| \leq R<\bar{R}$. Assume that $\max _{i}\left\|x_{i}(k)\right\| \leq \bar{R}$ for $k=n$ and consider $k=n+1$. From (7.4a) it is obvious that we have $\left\|s_{i}(k)\right\| \leq \bar{R}$ for each $i$ by the convexity of norm $\|\cdot\|$. Moreover,

$$
\begin{aligned}
\left\|x_{i}(n+1)\right\|^{2}= & \left\|s_{i}(n)+h\left[b_{i}(n)\left(P_{\mathcal{X}}\left(s_{i}(n)\right)-s_{i}(n)\right)-\alpha(n) g_{i}\left(s_{i}(n)\right)\right]\right\|^{2} \\
= & \left\|s_{i}(n)\right\|^{2}+h^{2} b_{i}^{2}(n)\left|s_{i}(n)\right|_{\mathcal{X}}^{2}+h^{2} \alpha^{2}(n)\left\|g_{i}\left(s_{i}(n)\right)\right\|^{2} \\
& +2 h b_{i}(n)\left\langle s_{i}(n), P_{\mathcal{X}}\left(s_{i}(n)\right)-s_{i}(n)\right\rangle \\
& -2 h b_{i}(n) \alpha(n)\left\langle s_{i}(n), g_{i}\left(s_{i}(n)\right)\right\rangle \\
& -2 h^{2} b_{i}(n) \alpha(n)\left\langle P_{\mathcal{X}}\left(s_{i}(n)\right)-s_{i}(n), g_{i}\left(s_{i}(n)\right)\right\rangle \\
\leq & \left\|s_{i}(n)\right\|^{2}+h^{2} \alpha^{2}(n) \bar{\gamma}^{2}-2 h \alpha(n)\left\langle s_{i}(n), g_{i}\left(s_{i}(n)\right)\right\rangle \\
& +2 h^{2} b \alpha(n) \bar{\gamma}\left\|s_{i}(n)\right\|
\end{aligned}
$$

where we use the facts that $\left\|g_{i}\left(s_{i}(n)\right)\right\| \leq \bar{\gamma},\left\langle s_{i}(n), P_{\mathcal{X}}\left(s_{i}(n)\right)-s_{i}(n)\right\rangle \leq-\left|s_{i}(n)\right|_{\mathcal{X}}^{2}$ by Lemma (3.4), and $h<2$. Now consider $\left\|s_{i}(n)\right\|$ in the following two cases.

1. $\left\|s_{i}(n)\right\| \leq R$. It follows from (7.8) and $h b \leq 1$ that

$$
\begin{aligned}
\left\|x_{i}(n+1)\right\|^{2} & \leq R^{2}+h^{2} \bar{\alpha}^{2} \bar{\gamma}^{2}+2 h \bar{\alpha} \bar{\gamma} R+2 h \bar{\alpha} \bar{\gamma} R \\
& \leq R^{2}+4 \bar{\alpha} \bar{\gamma} R+4 \bar{\alpha}^{2} \bar{\gamma}^{2}<\bar{R}^{2}
\end{aligned}
$$

2. $R<\left\|s_{i}(n)\right\| \leq \bar{R}$. By recalling the definition of subgradients and $(7.6)$ we have

$$
\begin{aligned}
\left\|x_{i}(n+1)\right\|^{2} & \leq \bar{R}^{2}+h^{2} \alpha^{2}(n) \bar{\gamma}^{2}+2 h^{2} \alpha(n) \bar{\gamma} \bar{R}-2 h \alpha(n) \delta \\
& \leq \bar{R}^{2}+h \alpha(n)\left(h \alpha(n) \bar{\gamma}^{2}+2 h \alpha(n) \bar{\gamma} \bar{R}-2 \delta\right) \leq \bar{R}^{2},
\end{aligned}
$$

if we notice that $h \leq \frac{2 \delta}{\bar{\gamma}^{2}+2 \bar{\gamma} \bar{R}}$ and $\bar{\alpha} \geq 1$.

By combining 1 and 2 we arrive at $\left\|x_{i}(n+1)\right\| \leq \bar{R}$ and $\max _{i}\left\|x_{i}(n+1)\right\| \leq \bar{R}$, which establishes the induction. 
2) Set Convergence. By $7.4 \mathrm{a}$ it is clear that

$$
\left|s_{i}(k)\right|_{\mathcal{X}} \leq\left(1-\eta D_{i}(k)\right)\left|x_{i}(k)\right|_{\mathcal{X}}+\eta \sum_{j \in \mathcal{N}_{i}} w_{i j}(k)\left|x_{j}(k)\right|_{\mathcal{X}}
$$

due to the convexity of set distance. Moreover, by $7.4 \mathrm{~b}$ we obtain that

$$
\begin{aligned}
\left|x_{i}(k+1)\right| \mathcal{X} & \leq\left(1-h b_{i}(k)\right)\left|s_{i}(k)\right|_{\mathcal{X}}+h \alpha(k)\left\|g_{i}\left(s_{i}(k)\right)\right\| \\
& \leq\left(1-h b_{i}(k)\right)\left|s_{i}(k)\right|_{\mathcal{X}}+h \alpha(k) \bar{\gamma} .
\end{aligned}
$$

Now combine $7.4 \mathrm{~b}$ and $7.4 \mathrm{a})$ into the following compact form

$$
\begin{aligned}
\Pi_{x}(k+1) & \leq(I-h B(k)) \Pi_{s}(k)+h \alpha(k) \bar{\gamma} \mathbf{1}_{N} \\
& \leq(I-h B(k))(I-\eta L(k)) \Pi_{x}(k)+h \alpha(k) \bar{\gamma} \mathbf{1}_{N},
\end{aligned}
$$

where $\Pi_{x}(k)=\left[\left|x_{1}(k)\right|_{\mathcal{X}}, \ldots,\left|x_{N}(k)\right|_{\mathcal{X}}\right]^{\prime}, \Pi_{s}(k)=\left[\left|s_{1}(k)\right|_{\mathcal{X}}, \ldots,\left|s_{N}(k)\right|_{\mathcal{X}}\right]^{\prime}$ and $B(k)=\operatorname{diag}\left\{b_{1}(k), \ldots, b_{N}(k)\right\}$.

Given a sequence of matrices $\{M(k)\}_{k=0}^{\infty}$, we denote the transition matrix $\Phi_{M}\left(k_{1}: k_{2}\right)=$ $M\left(k_{2}\right) \cdots M\left(k_{1}\right)$ for $k_{2}>k_{1}$, and $\Phi_{M}\left(k_{1}: k_{2}\right)=M\left(k_{1}\right)$ for $k_{1}=k_{2}$. Note that for any non-negative matrix $P,(I-h B(k)) P$ is still non-negative and $(I-h B(k)) P \leq P$ holds entry-wise. Moreover, if $B(k) \neq 0$ and $P$ is positive with $P^{\prime} \mathbf{1}_{N}=\mathbf{1}_{N}$, then the column sums of $(I-h B(k)) P$ are all smaller than 1 , or $\|(I-h B(k)) P\|_{1}<1$. By condition 1) of Theorem 7.1 we know that there exists $T_{1} \in \mathbb{N}^{+}$ such that $\mathcal{G}\left[k, k+T_{1}-1\right]$ is strongly connected for any $k$; therefore, each entry of $\Phi_{I-\eta L}(k, k+t)$ is positive when $t \geq N T_{1}-1$. By condition 2) we know that there exists $\tau \in[0, T-1]$ such that $B\left(k+N T_{1}-1+\tau\right) \neq 0$, and hence

$$
\left\|\left(I-h B\left(k+N T_{1}-1+\tau\right)\right) \Phi_{I-\eta L}\left(k: k+N T_{1}-1+\tau\right)\right\|_{1}<1 .
$$

By recalling the bounded edge weights in condition 1$)$, we can find a constant $\rho \in(0,1)$ such that

$$
\begin{aligned}
& \left\|\Phi_{(I-h B)(I-\eta L)}\left(k: k+N T_{1}+T-2\right)\right\|_{1} \\
\leq & \left\|\Phi_{(I-h B)(I-\eta L)}\left(k: k+N T_{1}+\tau-1\right)\right\|_{1} \\
\leq & \left\|\left(I-h B\left(k+N T_{1}-1+\tau\right)\right) \Phi_{I-\eta L}\left(k: k+N T_{1}-1+\tau\right)\right\|_{1} \leq \rho, \forall k .
\end{aligned}
$$

In consequence, $\left\|\Phi_{(I-h B)(I-\eta L)}\left(k_{1}: k_{2}\right)\right\|_{1} \leq C \rho^{k_{2}-k_{1}+1}$ holds for some constant $C$, and (7.13) can be seen as a stabilized system with diminishing disturbance $h \alpha(k) \bar{\gamma} \mathbf{1}_{N}$. The set convergence is thus established.

3) Consensus. Denote $x(k)=\operatorname{col}\left\{x_{1}(k), \cdots, x_{N}(k)\right\}$ and $\epsilon(k)=\operatorname{col}\left\{\epsilon_{1}(k), \cdots, \epsilon_{N}(k)\right\}$, where 
$\epsilon_{i}(k)=h\left[b_{i}(k)\left(P_{\mathcal{X}}\left(s_{i}(k)\right)-s_{i}(k)\right)-\alpha(k) g_{i}\left(s_{i}(k)\right)\right]$. With the update scheme $7.3 \mathrm{a}$ we can find the system dynamics as

$$
x(k+1)=\left((I-\eta L(k)) \otimes I_{m}\right) x(k)+\epsilon(k),
$$

and the consensus can be achieved by applying Lemma 6.16

4) Optimum set convergence. Denote $\Pi_{x}^{*}(k)=\left[\left|x_{1}(k)\right|_{\mathcal{X}^{*}}, \ldots,\left|x_{N}(k)\right|_{\mathcal{X}^{*}}\right]^{\prime}$ and $\Pi_{s}^{*}(k)=\left[\left|s_{1}(k)\right|_{\mathcal{X}^{*}}, \ldots,\left|s_{N}(k)\right| \mathcal{X}^{*}\right]^{\prime}$. Similar to 7.11, we get

$$
\Pi_{s}^{*}(k) \leq(I-\eta L(k)) \Pi_{x}^{*}(k)
$$

On the other hand, it follows analogously from (7.8) that

$$
\begin{aligned}
\left|x_{i}(k+1)\right|_{\mathcal{X}^{*}}^{2} \leq & \left\|x_{i}(k+1)-P_{\mathcal{X}^{*}}\left(s_{i}(k)\right)\right\|^{2} \\
\leq & \left|s_{i}(k)\right|_{\mathcal{X}^{*}}^{2}+h^{2} \alpha^{2}(k) \bar{\gamma}^{2}+2 h \alpha(k) \bar{\gamma}\left|s_{i}(k)\right|_{\mathcal{X}} \\
& -2 h \alpha(k)\left\langle s_{i}(k)-P_{\mathcal{X}^{*}}\left(s_{i}(k)\right), g_{i}\left(s_{i}(k)\right)\right\rangle \\
\leq & \left|s_{i}(k)\right|_{\mathcal{X}^{*}}^{2}+h^{2} \alpha^{2}(k) \bar{\gamma}^{2}+2 h \alpha(k) \bar{\gamma}\left|s_{i}(k)\right|_{\mathcal{X}} \\
& +2 h \alpha(k)\left[f_{i}\left(P_{\mathcal{X}^{*}}\left(s_{i}(k)\right)\right)-f_{i}\left(s_{i}(k)\right)\right] .
\end{aligned}
$$

From the set convergence and consensus results, we know that for any $\varepsilon>0$ there exists a time instant $k_{\varepsilon}$ such that

$$
\begin{aligned}
\max _{i}\left\{\left|x_{i}(k)\right|_{\mathcal{X}}\right\} & \leq \varepsilon, \\
\alpha(k) & \leq \varepsilon, \\
\max _{i}\left\{\left\|x_{i}(k)-\bar{x}(k)\right\|\right\} & \leq \varepsilon, \quad \forall k \geq k_{\varepsilon}
\end{aligned}
$$

where $\bar{x}(k)=\frac{1}{N} \sum_{i} x_{i}(k)$. From 7.4a it is clear that $\bar{s}(k)=\frac{1}{N} \sum_{i} s_{i}(k)=\bar{x}(k)$, and we can get

$$
\begin{aligned}
\max _{i}\left\{\left|s_{i}(k)\right| \mathcal{X}\right\} & \leq \varepsilon, \\
\alpha(k) & \leq \varepsilon, \\
\max _{i}\left\{\left\|s_{i}(k)-\bar{x}(k)\right\|\right\} & \leq \varepsilon, \quad \forall k \geq k_{\varepsilon},
\end{aligned}
$$

which leads to

$$
\left\|\bar{s}(k)-P_{\mathcal{X}}(\bar{x}(k))\right\| \leq \varepsilon
$$

and

$$
\left\|P_{\mathcal{X}^{*}}(\bar{x}(k))-P_{\mathcal{X}^{*}}\left(s_{i}(k)\right)\right\| \leq \varepsilon
$$


by respectively imposing the convexity of set distance and the non-expansive property of projection in Lemma (3.5. As a consequence, it yields that

$$
\begin{aligned}
& f_{i}\left(P_{\mathcal{X}^{*}}\left(s_{i}(k)\right)\right)-f_{i}\left(s_{i}(k)\right) \\
= & {\left[f_{i}\left(P_{\mathcal{X}^{*}}\left(s_{i}(k)\right)\right)-f_{i}\left(P_{\mathcal{X}^{*}}(\bar{x}(k))\right)\right]+\left[f_{i}\left(P_{\mathcal{X}^{*}}(\bar{x}(k))\right)-f_{i}\left(P_{\mathcal{X}}(\bar{x}(k))\right)\right] } \\
& +\left[f_{i}(\bar{x}(k))-f_{i}\left(s_{i}(k)\right)\right]+\left[f_{i}\left(P_{\mathcal{X}}(\bar{x}(k))\right)-f_{i}(\bar{x}(k))\right] \\
\leq & f_{i}\left(P_{\mathcal{X}^{*}}(\bar{x}(k))\right)-f_{i}\left(P_{\mathcal{X}}(\bar{x}(k))\right)+3 \bar{\gamma} \varepsilon .
\end{aligned}
$$

Now define $\chi_{s}(k)=\left\langle\Pi_{s}^{*}(k), \Pi_{s}^{*}(k)\right\rangle$ and $\chi_{x}(k)=\left\langle\Pi_{x}^{*}(k), \Pi_{x}^{*}(k)\right\rangle$. Since graph $\mathcal{G}(k)$ is balanced, we know that $\mathbf{1}_{N}$ is a left eigenvector of the stochastic matrix $I-\eta L(k)$ with respect to the unit eigenvalue. Moreover, it implies from Lemma 3.3 that $(I-\eta L(k))^{\prime}(I-\eta L(k)) \leq I$. Therefore, 7.15 lends itself to

$$
\chi_{s}(k) \leq\left\langle(I-\eta L(k)) \Pi_{x}^{*}(k),(I-\eta L(k)) \Pi_{x}^{*}(k)\right\rangle \leq \chi_{x}(k) .
$$

Furthermore, the combination of 7.16 and 7.21 leads to that

$$
\begin{aligned}
\chi_{x}(k+1) & \leq \chi_{s}(k)+h \alpha(k)\left[2 \sum_{i=1}^{N}\left[f_{i}\left(P_{\mathcal{X}^{*}}(\bar{x}(k))\right)-f_{i}\left(P_{\mathcal{X}}(\bar{x}(k))\right)\right]+\varepsilon_{1}\right] \\
& =\chi_{x}(k)+h \alpha(k)\left[2\left(F^{*}-F\left(P_{\mathcal{X}}(\bar{x}(k))\right)\right)+\varepsilon_{1}\right], \quad \forall k \geq k_{\varepsilon}
\end{aligned}
$$

where $F^{*}=F\left(P_{\mathcal{X}^{*}}(\bar{x}(k))\right)$ is the constrained optimum, and $\varepsilon_{1}=\varepsilon \bar{\gamma}(h \bar{\gamma}+2 h+6)$.

Next, we proceed to prove the optimal set convergence $7.3 \mathrm{~b}$ by showing that

$$
\lim _{k \rightarrow \infty} \chi_{x}(k)=0
$$

Firstly, we show that for any $\delta>0$, we can choose $\varepsilon_{1}=\delta$ and $k_{\delta} \geq k_{\varepsilon}$ such that $F\left(\bar{x}\left(k_{\delta}\right)\right) \leq$ $F^{*}+\delta$. If this is not the case, then $F(\bar{x}(k))>F^{*}+\delta$ for all $k \geq k_{\varepsilon}$, and it follows from $(7.23)$ that

$$
\chi_{x}(k+1) \leq \chi_{x}(k)-h \alpha(k) \delta
$$

As a result, we get $\chi_{x}\left(k_{n}+1\right) \leq \chi_{x}\left(k_{\delta}\right)-h \delta \sum_{k=k_{\delta}}^{k_{n}} \alpha(k)$, which implies $\chi_{x}\left(k_{n}+1\right)<0$ with a sufficiently large $k_{n}$ by the persistence condition that $\sum_{k=k_{0}}^{\infty} \alpha(k)=\infty$, leading to a contradiction.

Now we proceed to examine $\chi_{x}(k)$ for $k \geq k_{\delta}$. From the above result, we know that for any $\delta>0$, there exists $k_{\delta}$ such that $F\left(P_{\mathcal{X}}\left(\bar{x}\left(k_{\delta}\right)\right)\right) \leq F^{*}+\delta$. Also note that $|\bar{x}(k)|_{\mathcal{X}^{*}}^{2} \leq \epsilon(\delta)$ if $F\left(P_{\mathcal{X}}(\bar{x}(k))\right) \leq$ $F^{*}+\delta$, where $\lim _{\delta \rightarrow 0} \epsilon(\delta)=0$ as a result of the continuity of $F$ and the global boundedness of $\bar{x}(k)$. Obviously $k_{\delta}$ can be chosen such that $\left|\bar{x}\left(k_{\delta}\right)\right|_{\mathcal{X}^{*}}^{2} \leq \epsilon$ and $\left\|x_{i}(k)-\bar{x}(k)\right\|^{2} \leq \frac{1}{N} \epsilon$ for each $i$ when $k \geq k_{\delta}$. In this case we have $\chi_{x}\left(k_{\delta}\right) \leq 2(1+N) \epsilon$. Invoking 7.25 and $F\left(P_{\mathcal{X}}\left(\bar{x}\left(k_{\delta}\right)\right)\right) \leq F^{*}+\delta$, we get 
$\chi_{x}\left(k_{\delta}+1\right) \leq \chi_{x}\left(k_{\delta}\right)+\delta \alpha(k) \leq 2(1+N) \epsilon+\delta \alpha(k)$. If $F\left(P_{\mathcal{X}}\left(\bar{x}\left(k_{\delta}+1\right)\right)\right) \leq F^{*}+\delta$, then we similarly get $\chi_{x}\left(k_{\delta}+2\right) \leq 2(1+N) \epsilon$. Otherwise by recalling 7.25 we get $\chi_{x}\left(k_{\delta}+2\right) \leq \chi_{x}\left(k_{\delta}+1\right)-\delta \alpha(k) \leq$ $2(1+N) \epsilon+\delta \alpha(k)$. Therefore, with the above induction we can show that $\chi_{x}(k) \leq 2(1+N) \epsilon+\delta \alpha(k)$ for $k \geq k_{\delta}$, and we see that $\lim \sup _{k \rightarrow \infty} \chi_{x}(k) \leq 2(1+N) \epsilon$. The convergence of $\chi_{x}(k)$ to zero, or equivalently the convergence to the optimum set $\mathcal{X}^{*}$ is readily established by the arbitrariness of $\delta$.

Remark 7.3. As shown in the above proof, the convergence to the optimum set hinges upon (7.23), which relates the square sum of the distance gap of each state, $\Pi(k)$, to the cost gap $F(\bar{x}(k))-F^{*}$ at $\bar{x}(k)$. As revealed from (7.23), we see that once $F(\bar{x})$ is close to $F^{*}$, or $\bar{x}$ enters into a small neighborhood of $\mathcal{X}^{*}$, then each individual state will not only approach the neighborhood as a result of consensus, but also stay close to it afterwards. Moreover, the persistence condition ensures that the neighborhood can be arbitrarily small, which completes the proof. In comparison, the analysis in [69] is mainly based on studying the distance sum of each state from some fixed point in $\mathcal{X}^{*}$, and needs an additional square-summable condition $\sum_{k+0}^{\infty} \alpha^{2}(k)<+\infty$ for the convergence to the fixed point, instead of the convergence to the optimum set in our case.

\subsection{Convergence Rate Analysis}

In this section we shall analyze the convergence rate when the diminishing step size $\alpha(k)$ satisfies some more conditions in addition to (7.5), and the aggregate cost function $F$ is $\beta$-strongly convex over $\mathcal{X}$. Along a similar line as in the last section, we first investigate the rate of set convergence and consensus, then the rate of convergence to the constrained optimum in the case of strongly convex functions. The following proposition is about the rate of set convergence and consensus.

Proposition 7.1. Suppose conditions in Thoerem (7.1) hold, and further assume that

$$
\limsup _{k \rightarrow \infty} \frac{\alpha(k)}{\alpha(k+1)}=1
$$

Then the rate of set convergence and consensus can be found as

$$
\begin{aligned}
\left|x_{i}(k)\right|_{\mathcal{X}} & =O(\alpha(k)), \\
\left\|x_{i}(k)-\bar{x}(k)\right\| & =O(\alpha(k)), i=1, \ldots, N .
\end{aligned}
$$

Proof. We first study the set convergence rate. Using the same notations as in the proof of set convergence, we obtain from $(7.13)$ the following transition equation

$$
\tilde{\Pi}_{x}(k+1) \leq M(k) \tilde{\Pi}_{x}(k)+h \frac{\alpha(k)}{\alpha(k+1)} \bar{\gamma} \mathbf{1}_{N},
$$


where $\tilde{\Pi}_{x}(k)=\frac{\Pi_{x}(k)}{\alpha(k)}$ and $M(k)=(I-h B(k))(I-\eta L(k)) \frac{\alpha(k)}{\alpha(k+1)}$. In addition, from the proof we also know that $\left\|\Phi_{(I-h B)(I-\eta L)}\left(k_{1}: k_{2}\right)\right\|_{\infty} \leq C \rho^{k_{2}-k_{1}+1}$ holds for some constant $C$. By the given condition (7.26), there exists $k_{\rho}$ such that $\rho \frac{\alpha(k)}{\alpha(k+1)} \leq \tilde{\rho}<1$ for $k \geq k_{\rho}$. As a result, it holds for the transition matrix that

$$
\left\|\Phi_{M}\left(k_{1}: k_{2}\right)\right\|_{\infty}=\left\|\Phi_{(I-h B)(I-\eta L)}\left(k_{1}: k_{2}\right)\right\|_{\infty} \prod_{k=k_{1}}^{k_{2}} \frac{\alpha(k)}{\alpha(k+1)} \leq C \tilde{\rho}^{k_{2}-k_{1}+1},
$$

where $k_{1}, k_{2} \geq k_{\rho}$. Therefore, 7.28 can be seen as a stable system with bounded input, and we can find a constant $\tilde{C}$ so that $\tilde{\Pi}_{x}(k) \leq \tilde{C}$, or $\left|x_{i}(k)\right|_{\mathcal{X}}=O(\alpha(k))$ for each $i$. The consensus rate can be obtained similarly as in Section 6.4.2. In summary, we have proved (7.27).

Next, we study the rate of optimum set convergence by considering 77.23. As mentioned in Remark 7.3, 7.23 relates the square sum of distance gap to the cost gap. Therefore, if the cost gap can be quantified in terms of the distance gap, the rate of optimum set convergence can be characterized. In this light, we consider a strongly convex $F$. Moreover, in view of (7.26), we require that the diminishing step size $\alpha(k)$ further satisfy the condition

$$
\alpha(k)-\alpha(k+1)=O\left(\alpha^{2}(k)\right)
$$

Remark 7.4. Under condition (7.29) we know that $\alpha(k)-\alpha(k+1)=o(\alpha(k))$ and resultantly $\lim _{k \rightarrow \infty} \frac{\alpha(k+1)}{\alpha(k)}=1-\lim _{k \rightarrow \infty} \frac{\alpha(k)-\alpha(k+1)}{\alpha(k)}=1$, which is in accordance with 7.26. Furthermore, we can find $C>0$ such that $\frac{\alpha(k)-\alpha(k+1)}{\alpha(k) \alpha(k+1)} \leq C$ for sufficiently large $k$, i.e. $\frac{1}{\alpha(k+1)}-\frac{1}{\alpha(k)} \leq C$. Direct computation gives rise to that $\alpha(k) \geq \frac{1}{C\left(k-k_{0}+1\right)+1 / \alpha\left(k_{0}\right)}$ for some $k_{0} \in \mathbb{N}^{+}$, and the persistence condition is readily achieved.

Before presenting the main result, we introduce the following lemmas which play a key role in deriving convergence rate.

Lemma 7.1. If $\alpha(k)$ satisfies $\lim _{k \rightarrow \infty} \alpha(k)=0$ and 7.29 , then the following results hold true:

1) $\sum_{k=0}^{\infty} \frac{\alpha(k)}{\sum_{t=0}^{k+1} \alpha(t)}=+\infty$;

2) $\lim _{k \rightarrow \infty} \alpha(k) \sum_{t=0}^{k} \alpha(t)=0$ if $\liminf _{k \rightarrow \infty} \frac{\alpha(k)-\alpha(k+1)}{\alpha^{2}(k)}>0$.

Proof. 1) The result is reminiscent of $\int_{0}^{T} \frac{\alpha(t)}{1+\int_{0}^{t} \alpha(\tau) d \tau} d t=\ln \left(1+\int_{0}^{T} \alpha(T)\right)$ for a continuous $\alpha(t)$. 
Actually, we can find a small number $\varepsilon>0$ and $k_{\varepsilon}$ such that

$$
\begin{aligned}
& \ln \left(\sum_{t=0}^{k+1} \alpha(t)\right)-\ln \left(\sum_{t=0}^{k} \alpha(t)\right) \\
= & \ln \left(1+\frac{\alpha(k+1)}{\sum_{t=0}^{k} \alpha(t)}\right) \\
\leq & \frac{\alpha(k+1)}{\sum_{t=0}^{k} \alpha(t)} \leq(1+\varepsilon) \frac{\alpha(k)}{\sum_{t=0}^{k+1} \alpha(t)}, \forall k \geq k_{\varepsilon},
\end{aligned}
$$

if we take 7.26 into account. Taking summation from $k_{\varepsilon}$ to $k_{n}$ yields that

$$
\sum_{k=k_{\varepsilon}}^{k_{n}} \frac{\alpha(k)}{\sum_{t=0}^{k+1} \alpha(t)} \geq \frac{1}{1+\varepsilon}\left[\ln \left(\sum_{k=0}^{k_{n}+1} \alpha(k)\right)-\ln \left(\sum_{k=0}^{k_{\varepsilon}} \alpha(k)\right)\right]
$$

and 1) is readily achieved by letting $k_{n} \rightarrow \infty$ and considering (7.5).

2) Since $\liminf \operatorname{in}_{k \rightarrow \infty} \frac{\alpha(k)-\alpha(k+1)}{\alpha^{2}(k)}>0$, there exists $c>0$ such that $\frac{\alpha(k)-\alpha(k+1)}{\alpha(k) \alpha(k+1)} \geq c>0$ for sufficiently large $k$, and it holds that $\alpha(k) \leq \frac{1}{c\left(k-k_{0}+1\right)+1 / \alpha\left(k_{0}\right)}$ for some $k_{0} \in \mathbb{N}^{+}$. The result follows if we note that $\frac{1}{k} \leq \ln \frac{1}{1-1 / k}=\ln k-\ln (k-1)$ for $k>1$ and $\frac{1}{k_{n}} \sum_{k=k_{0}}^{k_{n}} \frac{1}{k} \leq \frac{1}{k_{n}}\left[\ln k_{n}-\ln \left(k_{0}-1\right)\right]$.

Lemma 7.2. [124] Given the following scalar system

$$
z(k+1) \leq(1-a(k)) z(k)+b(k)
$$

if $a(k) \in(0,1), \sum_{k=0}^{+\infty} a(k)=+\infty$ and $\lim _{k \rightarrow \infty} \frac{b(k)}{a(k)}$ exists, then it holds that

$$
\limsup _{k \rightarrow \infty} z(k) \leq \lim _{k \rightarrow \infty} \frac{b(k)}{a(k)}
$$

Theorem 7.2. Let the conditions in Theorem 7.1 hold, except that the persistence condition 7.5 is replaced by (7.29). Moreover, assume that the aggregate cost function $F$ is $\beta$-strongly convex over $\mathcal{X}$, and denote $x^{*}$ as the unique minimum of $F$ over $\mathcal{X}$. Then the minimization problem (7.3) can be solved, and the corresponding convergence rate for different step sizes is given as follows:

1) If $\lim \sup _{k \rightarrow \infty} r(k)<\frac{h \beta}{N}$, then $\left\|x_{i}(k)-x^{*}\right\|^{2}=O(\alpha(k))$;

2) If $r(k)=\frac{h \beta}{N}+O(\alpha(k))$, then $\left\|x_{i}(k)-x^{*}\right\|^{2}=O\left(\alpha(k) \sum_{t=0}^{k} \alpha(t)\right)$;

3) If $\frac{h \beta}{N} \leq \limsup _{k \rightarrow \infty} r(k)<p$ and $\liminf _{k \rightarrow \infty} r(k)>0$, then $\left\|x_{i}(k)-x^{*}\right\|^{2}=O\left(\alpha^{\frac{h \beta}{p N}}(k)\right)$

where $r(k)=\frac{\alpha(k)-\alpha(k+1)}{\alpha^{2}(k)}$. 
Remark 7.5. Note that Theorem 7.2 is in a similar spirit as the result in the continuous-time case, and we can also make a similar remark as Remark 6.9. We observe that Theorem 7.2 reveals an interesting relationship between the choice of decaying subgradient step size and the convergence rate, which is also closely related with the structure of the cost function and the number of agents, as well as the control gain h. Roughly speaking, the three cases considered in Theorem 7.2 correspond to the following three types of $\alpha(k): \alpha(k)>\frac{N}{h \beta k}, \alpha(k)=\frac{N}{h \beta k}$ and $\alpha(t)<\frac{N}{h \beta k}$. Note that step sizes with the form $\frac{c}{k^{\gamma}}, 0<\gamma \leq 1, c>0$ are covered by these cases. Moreover, similar to Remark 6.10, the theorem also implies that one of the optimal step sizes is given by $\alpha(k)=\frac{c}{k}$ with $c>\frac{N}{h \beta}$, and consequently $x_{i}(k)$ converges to the unique optimum $x^{*}$ at the rate of $O\left(\frac{1}{\sqrt{k}}\right)$.

Proof. Under given conditions, the convergence 7.3 is obvious if we apply Theorem 7.1 and note that the persistence condition has been proved in Remark 7.4 when 7.29 is satisfied. In the sequel we shall study the convergence rate for strongly convex functions with different types of $\alpha(k)$.

Again by Remark 7.4 we know that condition 7.26 is satisfied, and by Proposition 7.1 there exist $k_{1}$ and $C>0$ such that for $k \geq k_{1}$, it holds that

$$
\left\|x_{i}(k)-\bar{x}(k)\right\| \leq C \alpha(k),\left|x_{i}(k)\right|_{\mathcal{X}} \leq C \alpha(k),|\bar{x}(k)|_{\mathcal{X}^{*}}^{2} \leq C
$$

As a result, we obtain

$$
\begin{aligned}
& \left\|x_{i}(k)-x^{*}\right\|^{2} \\
= & \left\|x_{i}(k)-\bar{x}(k)+\bar{x}(k)-P_{\mathcal{X}}(\bar{x}(k))+P_{\mathcal{X}}(\bar{x}(k))-x^{*}\right\|^{2} \\
= & \left\|x_{i}(k)-\bar{x}(k)\right\|^{2}+\left\|\bar{x}(k)-P_{\mathcal{X}}(\bar{x}(k))\right\|^{2}+\left\|P_{\mathcal{X}}(\bar{x}(k))-x^{*}\right\|^{2} \\
& +2\left\langle x_{i}(k)-\bar{x}(k), \bar{x}(k)-P_{\mathcal{X}}(\bar{x}(k))\right\rangle+2\left\langle x_{i}(k)-\bar{x}(k), P_{\mathcal{X}}(\bar{x}(k))-x^{*}\right\rangle \\
& +2\left\langle P_{\mathcal{X}}(\bar{x}(k))-x^{*}, P_{\mathcal{X}}(\bar{x}(k))-x^{*}\right\rangle \\
\leq & \left\|P_{\mathcal{X}}(\bar{x}(k))-x^{*}\right\|^{2}+2 C^{2} \alpha(k)\left(1+\frac{1}{N}\right)+C^{2} \alpha^{2}(k)\left(1+\frac{1}{N^{2}}+\frac{2}{N}\right),
\end{aligned}
$$

which leads to that

$$
-\left\|P_{\mathcal{X}}(\bar{x}(k))-x^{*}\right\|^{2} \leq-\frac{1}{N} \chi_{x}(k)+2 C^{2} \alpha(k)\left(1+\frac{1}{N}\right)+C^{2} \alpha^{2}(k)\left(1+\frac{1}{N^{2}}+\frac{2}{N}\right) .
$$

Note that now $\chi_{x}(k)=\sum_{i=1}^{N}\left\|x_{i}(k)-x^{*}\right\|^{2}$. In a similar spirit of 7.21, we get

$$
f_{i}\left(x^{*}\right)-f_{i}\left(s_{i}(k)\right) \leq\left(f_{i}\left(x^{*}\right)-f_{i}\left(P_{\mathcal{X}}(\bar{x}(k))\right)\right)+3 \bar{\gamma} C \alpha(k)
$$


By substituting (7.21 into 7.16 and summing over $i \in \mathcal{N}$, it yields that

$$
\begin{aligned}
\chi_{x}(k+1) \leq & \chi_{x}(k)+2 h \alpha(k)\left(F\left(x^{*}\right)-F\left(P_{\mathcal{X}}(\bar{x}(k))\right)\right) \\
& +\alpha^{2}(k) h \bar{\gamma}(h \bar{\gamma}+2+6 C) .
\end{aligned}
$$

Now by combining 7.32 and 7.23 , and applying Lemma 3.9 to $F$ we arrive at

$$
\begin{aligned}
\chi_{x}(k+1) & \leq \chi_{x}(k)-h \beta \alpha(k)\left\|P_{\mathcal{X}}(\bar{x}(k))-x^{*}\right\|^{2}+\alpha^{2}(k) h \bar{\gamma}(h \bar{\gamma}+2+6 C) \\
& \leq\left(1-\frac{h \beta}{N} \alpha(k)\right) \chi_{x}(k)+\Theta(k),
\end{aligned}
$$

where $\Theta(k)=h \alpha^{2}(k)\left[4 C^{2} \beta+\bar{\gamma}(h \bar{\gamma}+2+6 C)\right]+4 h \alpha^{3}(k) C^{2} \beta=O\left(\alpha^{2}(k)\right)$. Based on 7.33 we shall analyze the convergence rate of $\chi_{x}(k)$ in three cases, with $\tilde{r}(k) \triangleq \frac{\alpha(k)-\alpha(k+1)}{\alpha(k) \alpha(k+1)}$.

1) $\lim \sup _{k \rightarrow \infty} r(k)<\frac{h \beta}{N}$. In this case, for any $\varepsilon>0$ there must exist $k_{\varepsilon}$ such that $\sup _{k \geq k_{\varepsilon}} \tilde{r}(k)(1-$ $\left.\frac{h \beta}{N} \alpha(k)\right) \leq \frac{h \beta}{N}-\varepsilon$. Define $\tilde{\chi}_{x}(k)=\frac{\chi_{x}(k)}{\alpha(k)}$, then by 7.33 one can find that $\forall k \geq k_{\varepsilon}$,

$$
\begin{aligned}
\tilde{\chi}_{x}(k+1) & \leq \frac{\alpha(k)}{\alpha(k+1)}\left(1-\frac{h \beta}{N} \alpha(k)\right) \tilde{\chi}_{x}(k)+\frac{\Theta(k)}{\alpha(k+1)} \\
& =(1+\alpha(k) \tilde{r}(k))\left(1-\frac{h \beta}{N} \alpha(k)\right) \tilde{\chi}_{x}(k)+\frac{\Theta(k)}{\alpha(k+1)} \\
& \leq(1-\varepsilon \alpha(k)) \tilde{\chi}_{x}(k)+\frac{\Theta(k)}{\alpha(k+1)} .
\end{aligned}
$$

Now we apply Lemma 7.2 to 7.34 and find that

$$
\limsup _{k \rightarrow \infty} \tilde{\chi}_{x}(k) \leq \lim _{k \rightarrow \infty} \frac{\Theta(k)}{\varepsilon \alpha(k) \alpha(k+1)} \leq \frac{1}{\varepsilon} h\left[4 C^{2} \beta+\bar{\gamma}(h \bar{\gamma}+2+6 C)\right],
$$

which implies that $\chi_{x}(k)=O(\alpha(k))$.

2) $r(k)=\frac{h \beta}{N}+O(\alpha(k)) \leq \frac{h \beta}{N}+c_{1} \alpha(k)$ for some $c_{1}>0$. In this case, we can find that

$$
\begin{aligned}
\tilde{r}(k) & \leq \frac{\frac{h \beta}{N} \alpha^{2}(k)+c_{1} \alpha^{3}(k)}{\alpha(k) \alpha(k+1)} \\
& =\left(\frac{h \beta}{N}+c_{1} \alpha(k)\right)(1+\tilde{r}(k) \alpha(k)) \\
& =\frac{h \beta}{N}+O(\alpha(k)) .
\end{aligned}
$$


Define $\tilde{\chi}_{x}(k)=\frac{\chi_{x}(k)}{\alpha(k) \sigma(k)}$ with $\sigma(k)=\sum_{t=0}^{k} \alpha(t)$, then by 7.33 we have

$$
\begin{aligned}
\tilde{\chi}_{x}(k+1) & \leq \frac{\alpha(k) \sigma(k)}{\alpha(k+1) \sigma(k+1)}\left(1-\frac{h \beta}{N} \alpha(k)\right) \tilde{\chi}_{x}(k)+\frac{\Theta(k)}{\alpha(k+1) \sigma(k+1)} \\
& =\left(1-\frac{h \beta}{N} \alpha(k)\right)\left(1+\tilde{r}(k) \alpha(k)-\frac{\alpha(k)}{\sigma(k+1)}\right) \tilde{\chi}_{x}(k)+\frac{\Theta(k)}{\alpha(k+1) \sigma(k+1)} \\
& \leq\left(1-\frac{\alpha(k)}{\sigma(k+1)}+\tilde{\alpha}_{1}(k)\right) \tilde{\chi}_{x}(k)+\frac{\Theta(k)}{\sigma(k+1)},
\end{aligned}
$$

where $\tilde{\alpha}_{1}(k)=-\frac{h \beta}{N} \tilde{r}(k) \alpha^{2}(k)+O\left(\alpha^{2}(k)\right)+\frac{h \beta}{N} \frac{\alpha^{2}(k)}{\sigma(k+1)}=o\left(\frac{\alpha(k)}{\sigma(k+1)}\right)$. Here we used the fact that $\alpha^{2}(k)=o\left(\frac{\alpha(k)}{\sigma(k+1)}\right)$ by recalling $\lim _{k \rightarrow \infty} \alpha(k) \sigma(k)=0$ in Lemma 7.1. Now we apply Lemma 7.2 to obtain that

$$
\limsup _{k \rightarrow \infty} \tilde{\chi}_{x}(k) \leq \lim _{k \rightarrow \infty} \frac{\frac{\Theta(k)}{\alpha(k+1) \sigma(k+1)}}{\frac{\alpha(k)}{\sigma(k+1)}-\tilde{\alpha}_{1}(k)}=h\left[4 C^{2} \beta+\bar{\gamma}(h \bar{\gamma}+2+6 C)\right],
$$

which implies $\chi_{x}(k)=O\left(\alpha(k) \sum_{t=0}^{k} \alpha(t)\right)$.

3) $\frac{h \beta}{N} \leq \limsup _{k \rightarrow \infty} r(k)<p$. There exists a time instant $k_{p}$ such that $\tilde{r}(k)<p, \forall k \geq k_{\varepsilon}$. Define $\tilde{\chi}_{x}(k)=\frac{\chi_{x}(k)}{\alpha^{\frac{h \beta}{p N}}(k)}$, then by 7.33 we find the following result for sufficiently large $k$ :

$$
\begin{aligned}
\tilde{\chi}_{x}(k+1) & =(1+\tilde{r}(k) \alpha(k))^{\frac{h \beta}{p N}}\left(1-\frac{h \beta}{N} \alpha(k)\right) \tilde{\chi}_{x}(k)+\frac{\Theta(k)}{\alpha^{\frac{h \beta}{p N}}(k+1)} \\
& \leq\left[1-\frac{h \beta}{N} \alpha^{2}(k)+o\left(\alpha^{2}(k)\right)\right] \tilde{\chi}_{x}(k)+\frac{\Theta(k)}{\alpha^{\frac{h \beta}{p N}}(k+1)} \\
& \leq \tilde{\chi}_{x}(k)+\frac{\Theta(k)}{\alpha^{\frac{h \beta}{p N}}(k+1)} .
\end{aligned}
$$

By $\Theta(k)=O\left(\alpha^{2}(k)\right)$ and $\lim _{k \rightarrow \infty} \frac{\alpha(k)}{\alpha(k+1)}=1$, we know that $\frac{\Theta(k)}{\alpha^{\frac{h \beta}{p N}}(k+1)}=O\left(\alpha^{2-\frac{h \beta}{p N}}(k)\right)$. Since $\liminf \inf _{k \rightarrow \infty} r(k)>$ 0 , we can find positive constants $c$ and $k_{0} \in \mathbb{N}^{+}$so that $\alpha(k) \leq \frac{1}{c\left(k-k_{0}+1\right)+1 / \alpha\left(k_{0}\right)}$ for $k \geq k_{0}$. In consequence, $\sum_{k=0}^{\infty} \alpha^{2-\frac{h \beta}{p N}}(k)<\infty$ as $2-\frac{h \beta}{p N}>1$, and $\tilde{\chi}_{x}(k)<\infty$, which implies that $\chi_{x}(k)=$ $O\left(\alpha^{\frac{h \beta}{p N}}(k)\right)$.

Remark 7.6. Theorem 7.2 shows that the convergence rate is closely dependent on the step size $\alpha(t)$. From the proof it is clear that the analysis is built upon (7.33), which is a linear time-varying system of $\chi_{x}(k)$ describing the dynamics of aggregate squared distance from each agent to the optimum point. Then we proceed further to study the dynamics of scaled distance $\tilde{\chi}_{x}(k)$ under different conditions, as seen respectively in (7.34, (7.36) and 7.38). By applying Lemma 7.2 we are able to obtain an asymptotic upper bound of the convergence rate for different step sizes, which is a major difference from existing works where the upper bound can only be obtained for specific step sizes, say $\alpha(k)=O\left(\frac{1}{\sqrt{k}}\right)$ in [11, 72] and $\alpha(k)=O\left(\frac{1}{k}\right)$ in 125 .

Remark 7.7. With the bounded subgradient we readily obtain the optimal value residual as $\mid F(\bar{x}(k))-$ 
$F\left(x^{*}\right) \mid=O\left(\frac{1}{\sqrt{k}}\right)$, if we take the step size as $\alpha(k)=\frac{c}{k}$ with $c>\frac{N}{h \beta}$. When compared with the existing works on distributed optimization which address non-smooth costs by subgradient methods, this result outperforms the ergodic rate of $O\left(\frac{\log k}{\sqrt{k}}\right)$ in [71] and [126] with the additional strong convexity condition. When compared with [125] which addresses strongly convex costs by using running average with stochastic subgradients, our result provides a sharper upper bound of optimum residual $\left\|x_{i}(k)-x^{*}\right\|$ than 125. where the convergence rate is given by $O\left(\sqrt{\frac{\log k}{k}}\right)$. Although the convergence of the optimal value residual is slower than $\frac{\log k}{k}$ in [125], it should be noted that many optimization problems need to solve the optimum instead of the optimal value, such as the least-squares problem and the optimal rendezvous point problem in the MAS context.

\subsection{Further Discussion for a Fixed Network}

In this section we shall explore the necessary and sufficient conditions to guarantee the discrete-time constrained optimal consensus under a fixed communication network, as well as the convergence in the presence of fixed communication delays.

\subsubsection{Necessary and Sufficient Conditions}

Theorem 7.3. Consider MAS (7.1) with the distributed control protocol (7.4) and $\max _{i}\left\|x_{i}(0)-z\right\| \leq$ $R$ for some $z \in \mathcal{X}$. Under Assumptions 7.1 and 7.2 and a fixed communication topology, there exists $\hat{h}$ such that the constrained optimal consensus $(7.3)$ can be achieved with any $h \in(0, \hat{h})$ for any arbitrary cost function and constraint set, if and only if

1) At least one agent can access $\mathcal{X}$;

2) The graph $\mathcal{G}$ is balanced and contains a spanning tree;

3) $\alpha(k)$ is diminishing, and satisfies the persistence condition (7.5).

Proof. The sufficiency has already been proved in Theorem 7.1 where fixed topology is only a special case. For the necessity, we shall prove it in the following steps: 1) At least one agent knows $\mathcal{X}$; 2) The graph contains a spanning tree; 3) $\left.\left.\lim _{k \rightarrow \infty} \alpha(k)=0 ; 4\right) \sum_{k=0}^{\infty} \alpha(k)=\infty ; 5\right)$ The graph is balanced.

1) Obvious.

2) By contradiction, we assume that $\mathcal{G}$ does not contain a spanning tree. Then by 127 we know that the nodes can be divided into two groups without communication between them, and the consensus cannot be achieved by recalling the necessity proof in 127 . 
3) By contradiction, assume that we can find a subsequence $\left\{k_{l}\right\}_{l=1}^{\infty}$ such that $\alpha\left(k_{l}\right) \geq \bar{\alpha}_{0}>0$. Let $F$ be a continuously differentiable and strictly convex function, and we denote its global and constrained optimum respectively as $x_{0}^{*}$ and $x^{*}$. Assume that $x_{0}^{*} \neq x^{*}$, and consider the single agent case. Now the update scheme (7.4) becomes

$$
x(k+1)=x(k)+h\left[b\left(P_{\mathcal{X}}(x(k))-x(k)-\alpha(k) \nabla F(x(k))\right] .\right.
$$

Given $x(k) \rightarrow x^{*}$ and $\varepsilon>0$, we can find $k_{\varepsilon}$ such that $\left\|x(k)-x^{*}\right\|<\varepsilon$ and $\left\|P_{\mathcal{X}}(x(k))-x(k)\right\|<\varepsilon$ for $k \geq k_{\varepsilon}$. Select $k_{l} \geq k_{\varepsilon}$ and $\alpha\left(k_{l}\right) \geq \bar{\alpha}_{0}$. Since $x^{*}$ is not the global optimum, there exists $\gamma_{0}>0$ such that $\|\nabla F(x)\| \geq \gamma_{0}$ for $x \in B\left(x^{*}, \varepsilon\right)$ when $\varepsilon$ is sufficiently small. As a result, we have

$$
\begin{aligned}
\left\|x\left(k_{l}+1\right)-x^{*}\right\| & \geq\left\|h \alpha\left(k_{l}\right) \nabla F\left(x\left(k_{l}\right)\right)\right\|-\left\|x\left(k_{l}\right)-x^{*}\right\|-h b\left\|P_{\mathcal{X}}(x(k))-x(k)\right\| \\
& \geq h \alpha_{0} \gamma_{0}-\varepsilon-h b \varepsilon,
\end{aligned}
$$

which is in contradiction with the convergence to $x^{*}$. Therefore, $\lim _{k \rightarrow \infty} \alpha(k)=0$.

4) We consider the case when $\mathcal{X}=\mathbb{R}^{m}$ and the initial condition satisfies $x_{i}(0)=x_{0} \notin \mathcal{X}^{*}, \forall i \in \mathcal{N}$. With the identical initial values we know that Under the convergence assumption, all states are globally bounded and we can find $\bar{\gamma}>0$ as an upper bound of the subgradients $g_{i}\left(s_{i}(k)\right)$ for all $k$. In view of the control law (7.4), we have $\left\|x_{i}(1)-x_{0}\right\| \leq h \bar{\gamma} \alpha(0)$ due to the averaging step. By induction it is easy to see that $\left\|x_{i}(k+1)-x_{0}\right\| \leq h \bar{\gamma} \sum_{t=0}^{k} \alpha(t)$ for all $k$. Since $x_{0} \notin \mathcal{X}^{*}$, we have $\left|x_{0}\right| \mathcal{X}^{*}=\delta>0$. If $\sum_{k=0}^{\infty} \alpha(k)<+\infty$, then for sufficiently small $h$, it results in $\lim _{k \rightarrow \infty}\left|x_{i}(k)\right|_{\mathcal{X}^{*}} \geq \frac{\delta}{2}$, which contradicts with the optimum convergence $7.3 \mathrm{~b}$.

5) Before proving the balance condition, we first show that the graph is strongly connected by contradiction. From step 2) we know that the graph $\mathcal{G}$ must contain a spanning tree, and we can find the maximal strongly connected subgraph of $\mathcal{G}$ and accordingly partition the graph into two parts since $\mathcal{G}$ is not strongly connected. More precisely, according to [128], the matrix $I-\eta L$ is reducible, i.e. there is a permutation matrix $P$ such that

$$
P(I-\eta L) P^{\prime}=\left[\begin{array}{cc}
I-h L_{1} & 0 \\
* & I-h L_{2}
\end{array}\right],
$$

where $L_{1} \in \mathbb{R}^{N_{1} \times N_{1}}, L_{2} \in \mathbb{R}^{\left(N-N_{1}\right) \times\left(N-N_{1}\right)}$ and $I-\eta L_{1}$ is an irreducible matrix. In this case the subgraph $\mathcal{G}_{1}$ induced by $L_{1}$ is strongly connected, and there exists a positive vector $\omega=\left[\omega_{1}, \cdots, \omega_{N_{1}}\right]^{\prime} \in$ $\mathbb{R}^{N_{1}}$ satisfying $w^{\prime} L_{1}=0$ and $w^{\prime} \mathbf{1}_{N_{1}}=1$. Define $F_{\omega}(x)=\sum_{i=1}^{N_{1}} \omega_{i} f_{v_{i}}(x), \mathcal{X}_{\omega}^{*}=\left\{v \mid \arg \min _{v \in \mathcal{X}} F_{\omega}(v)\right\}$ and

$$
\begin{aligned}
& \chi_{w, x}(k)=\sum_{i=1}^{N_{1}} w_{i}\left|x_{v_{i}}(k)\right|_{\mathcal{X}_{\omega}^{*}}^{2}=\left\langle P \Pi_{\omega, x}^{*}(k), R P \Pi_{\omega, x}^{*}(k)\right\rangle, \\
& \chi_{\omega, s}(k)=\left\langle P \chi_{\omega, s}(k), R P \chi_{\omega, s}(k)\right\rangle,
\end{aligned}
$$


where $\left[v_{1}, \cdots, v_{N}\right]=[1, \cdots, N] P^{\prime}, R=\operatorname{diag}\left\{\omega_{1}, \cdots, \omega_{N_{1}}, 0, \cdots, 0\right\} \in \mathbb{R}^{N \times N}$ and

$$
\begin{aligned}
& \Pi_{\omega, x}^{*}(k)=\operatorname{col}\left\{\left|x_{1}(k)\right|_{\mathcal{X}_{\omega}^{*}}, \cdots,\left|x_{N}(k)\right|_{\mathcal{X}_{\omega}^{*}}\right\} \\
& \Pi_{\omega, s}^{*}(k)=\operatorname{col}\left\{\left|s_{1}(k)\right|_{\mathcal{X}_{\omega}^{*}}, \cdots,\left|s_{N}(k)\right|_{\mathcal{X}_{\omega}^{*}}\right\} .
\end{aligned}
$$

Noticing that $\left[\omega_{1}, \cdots, \omega_{N_{1}}, 0, \cdots, 0\right]^{\prime}$ is a left eigenvector of $P(I-\eta L) P^{\prime}$ with respect to the eigenvalue 1 , it yields from Lemma 3.3 that

$$
P(I-\eta L)^{\prime} P^{\prime} R P(I-\eta L) P^{\prime} \leq R .
$$

As a result, we get

$$
(I-\eta L)^{\prime} P^{\prime} R P(I-\eta L)=P^{\prime} P(I-\eta L)^{\prime} P^{\prime} R P(I-\eta L) P^{\prime} P \leq P^{\prime} R P .
$$

Similar to 7.15 we get $\Pi_{\omega, s}^{*}(k) \leq(I-\eta L) \Pi_{\omega, x}^{*}(k)$, which can be combined with 7.40$)$ and $(7.41)$ to yield that

$$
\begin{aligned}
\chi_{\omega, s}(k+1) & \leq\left\langle P(I-\eta L) \Pi_{\omega, x}^{*}(k), R P(I-\eta L) \Pi_{\omega, x}^{*}(k)\right\rangle \\
& \leq\left\langle P \Pi_{\omega, x}^{*}(k), R P \Pi_{\omega, x}^{*}(k)\right\rangle=\chi_{\omega, x}(k) .
\end{aligned}
$$

We know from step 3) that $\lim _{k \rightarrow \infty} \alpha(k)=0$, which gives rise to 7.21 in the proof of Theorem 7.1 by combining set convergence and consensus. Hence we arrive at

$$
\chi_{\omega, x}(k+1) \leq \chi_{\omega, x}(k)+2 h \alpha(k)\left[F_{\omega}\left(P_{\mathcal{X}^{*}}(\bar{x}(k))\right)-F_{\omega}\left(P_{\mathcal{X}}(\bar{x}(k))\right)+\varepsilon_{1}\right]
$$

Noticing that 7.43 is reminiscent of 7.23 , we can proceed along a similar line as in the proof of Theorem 7.1 to show that $x_{i}(k)$ asymptotically converges to the set $\mathcal{X}_{\omega}^{*}$ under the persistence condition which has been proved in steps 3) and 4). On the other hand, there always exists some cost function $F$ such that $\mathcal{X}_{\omega}^{*} \cap \mathcal{X}^{*}=\emptyset$, which leads to a contradiction with $\lim _{k \rightarrow \infty}\left|x_{i}(k)\right|_{\mathcal{X}^{*}}=0$. Therefore, we conclude that the graph is strongly connected.

Next, we assume that the graph is not balanced. Similar analysis can be applied by letting $P=I$, $N_{1}=N$ and $L_{1}=L$ in 7.39 . Since the graph is not balanced, we know that $\omega \notin \operatorname{span}\left\{\mathbf{1}_{N}\right\}$ which leads to the existence of cost functions such that $\mathcal{X}_{\omega}^{*} \cap \mathcal{X}^{*}=\emptyset$, which is another contradiction.

Remark 7.8. The necessary conditions 2) and 3) are respectively about the fixed communication graph and the step size of subgradients. It is well known that under a fixed topology and the simple averaging scheme (7.4a), average consensus can only be achieved if the graph is balanced and contains a spanning tree, otherwise the final consensus value is a weighted average of initial values. We see a similar result from the step 5) in the above proof, which highlights the effect of communication graph on the final consensus value. For the subgradient step size $\alpha(k)$, it has to be diminishing and not 
summable. Intuitively, it suggests that to ensure the convergence to the constrained optimum set, the effect of subgradient can not dominate that of projection and averaging for the constrained consensus, and yet needs to be everlasting to achieve the minimization.

\subsubsection{Convergence under Fixed Time Delays}

In networked systems, the communication between different agents is typically subject to delays. In this section, we consider a fixed delay $d_{i j}$ for each transmission channel $(j, i)$ under a fixed topology. Denoting $y_{i j}$ as the state information that the agent $i$ receives from its neighbor $j$ at time step $k$, we have

$$
y_{i j}(k)=x_{j}\left(k-d_{i j}\right)
$$

Furthermore, we assume that each agent can always obtain its own information in time, namely $d_{i i}=0$ for all $i$. To achieve the optimal consensus within the constraint set, we revise the control protocol (7.4) by using the delayed information as follows:

$$
\begin{aligned}
s_{i}(k) & =x_{i}(k)+\eta \sum_{j \in \mathcal{N}_{i}} w_{i j}\left(x_{j}\left(k-d_{i j}\right)-x_{i}(k)\right), \\
x_{i}(k+1) & =s_{i}(k)+h\left[b_{i}\left(P_{\mathcal{X}}\left(s_{i}(k)\right)-s_{i}(k)\right)-\alpha(k) g_{i}\left(s_{i}(k)\right)\right] .
\end{aligned}
$$

Note that the edge weight on channel $(j, i)$ is a constant $w_{i j}$ under the fixed topology, and $b_{i}=b>0$ iff agent $i$ can access $\mathcal{X}$. The above algorithm can be rewritten as

$$
\begin{aligned}
x_{i}(k+1)=x_{i}(k)+\eta \sum_{j \in \mathcal{N}_{i}} w_{i j}\left(x_{j}\left(k-d_{i j}\right)-\right. & \left.x_{i}(k)\right) \\
& +h\left[b_{i}\left(P_{\mathcal{X}}\left(s_{i}(k)\right)-s_{i}(k)\right)-\alpha(k) g_{i}\left(s_{i}(k)\right)\right] .
\end{aligned}
$$

Below we shall show that the presence of fixed time delays has no effect on the final convergence, nor the design of control parameters. More explicitly, we present the result as below:

Theorem 7.4. Given that $\max _{i}\left\|x_{i, 0}-z\right\| \leq R$ for some $z \in \mathcal{X}$, there exist positive constants $\bar{\eta}$ and $\bar{h}$ such that the constrained optimal consensus problem $(\overline{7.3)}$ in the presence of fixed communication delays can be solved by the two-step update algorithm 7.45 with $\eta \in(0,1 / \bar{D}), h \in(0, \bar{h})$ and $b \in(0,1)$, if the following two conditions are satisfied:

1. The graph $\mathcal{G}$ is balanced and has a spanning tree, with $\mathcal{X}$ accessible by at least one agent;

2. $\alpha(k)$ is diminishing, and satisfies the persistence condition 7.5 . 


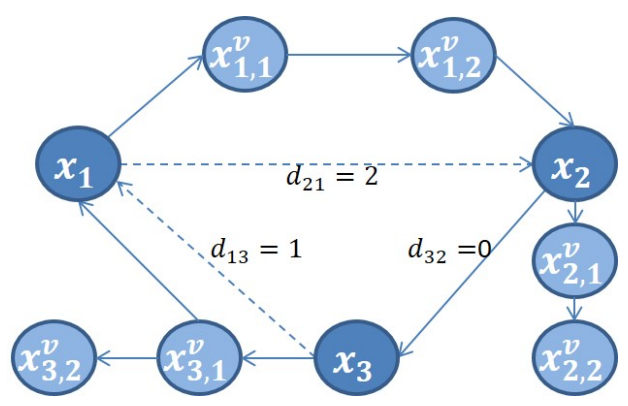

Figure 7.1: Construction of virtual nodes.

Note that $\bar{h}$ can be selected by the same procedure as in Remark 7.2. and $\bar{D}$ is the maximum degree.

Proof. As with the delay-free case, the convergence analysis will proceed along a similar line as in Section 7.2 However, to deal with the time delay issue, we need to introduce additional virtual nodes. Specifically, with the maximum step of delay denoted as

$$
\bar{d}=\max _{i, j} d_{i j}
$$

we construct virtual nodes $v_{i, d}$ 's for each $i$, with $d=1, \ldots, \bar{d}$ representing different steps of time delay. The state of each virtual node at time instant $k$ is denoted by $x_{i, d}^{v}(k)$, whose update is given by

$$
\begin{aligned}
s_{i, d}^{v}(k) & =x_{i, d-1}^{v}(k), \\
x_{i, d}^{v}(k+1) & =s_{i, d}^{v}(k),
\end{aligned}
$$

where $x_{i, 0}^{v}(k)=x_{i}(k)$. Note that 7.48) is reminiscent of 7.45 if we regard the virtual node $v_{i, d}$ as one that is not connected to the constraint set $\mathcal{X}$ and has a zero cost function. As shown more clearly in Fig. 7.1. node $v_{i, d}$ only relays the information from node $v_{i, d-1}$, and consequently $x_{i, d}^{v}(k)=x_{i}(k-d)$. Below we make use of these virtual nodes for the convergence analysis.

1) The global boundedness of the system can be shown similarly as in the delay-free case, if we notice that the time delay is only involved in the averaging step 7.45a).

2) Constraint set convergence. By $7.45 \mathrm{a}$ it is clear that

$$
\left|s_{i}(k)\right|_{\mathcal{X}} \leq\left(1-\eta D_{i}\right)\left|x_{i}(k)\right|_{\mathcal{X}}+\eta \sum_{j \in \mathcal{N}_{i}} w_{i j}\left|x_{j}\left(k-d_{i j}\right)\right|_{\mathcal{X}}
$$

due to the convexity of set distance, and the fact that $\left|s_{i, d}^{v}(k)\right|_{\mathcal{X}}=\left|x_{i, d-1}^{v}(k)\right|_{\mathcal{X}}$ by 7.48a. Define

$$
\begin{aligned}
\Pi_{s}(k) & =\left[\left|s_{1}(k)\right|_{\mathcal{X}}, \ldots,\left|s_{N}(k)\right|_{\mathcal{X}}\right]^{\prime}, \\
\Pi_{s, 0: \bar{d}}(k) & =\operatorname{col}\left\{\Pi_{s}(k), \Pi_{s, 1}^{v}(k), \ldots, \Pi_{s, \bar{d}}^{v}(k)\right\},
\end{aligned}
$$


where $\Pi_{s, d}^{v}(k)=\left[\left|s_{1, d}^{v}(k)\right|_{\mathcal{X}}, \ldots,\left|s_{N, d}^{v}(k)\right|_{\mathcal{X}}\right]^{\prime}$ for $d=1, \ldots, \bar{d}$; analogously, we can obtain $\Pi_{x}(k)$ and $\Pi_{x, 0: \bar{d}}(k)$ by replacing $s$ with $x$ on the right hand side respectively. Now 7.49 can be put in a vector form as

$$
\Pi_{s}(k) \leq\left[I-\eta\left(D-A_{0}\right) \eta A_{1} \cdots \eta A_{\bar{d}}\right] \Pi_{x, 1: \bar{d}}(k) .
$$

On the other hand, it yields from $7.45 \mathrm{~b}$ that

$$
\begin{aligned}
\left|x_{i}(k+1)\right|_{\mathcal{X}} & \leq\left\|x_{i}(k+1)-P_{\mathcal{X}}\left(s_{i}(k)\right)\right\| \\
& =\left\|\left(1-h b_{i}\right)\left(s_{i}(k)-P_{\mathcal{X}}\left(s_{i}(k)\right)\right)-\alpha(k) g_{i}\left(s_{i}(k)\right)\right\| \\
& \leq\left(1-h b_{i}\right)\left|s_{i}(k)\right|_{\mathcal{X}}+h \alpha(k) \bar{\gamma} .
\end{aligned}
$$

By combining 77.51 and $(7.12$ we find the following augmented system

$$
\Pi_{x, 0: \bar{d}}(k+1) \leq \Xi_{B} \Pi_{x, 0: \bar{d}}(k)+h \alpha(k) \Gamma,
$$

where $\Xi_{B}=\Lambda_{B} \Xi$ with

$$
\begin{aligned}
& \Xi=\left[\begin{array}{ccccc}
I-\eta\left(D-A_{0}\right) & \eta A_{1} & \cdots & \eta A_{\bar{d}-1} & \eta A_{\bar{d}} \\
I & & & & \\
& \ddots & & & \\
& & I & &
\end{array}\right], \\
& \Lambda_{B}=\operatorname{diag}(I-h B, I, \ldots, I), \\
& B=\operatorname{diag}\left(b_{1}, \ldots, b_{N}\right), \\
& \Gamma=\operatorname{col}\left\{\bar{\gamma} \mathbf{1}_{N}, 0, \cdots, 0\right\} .
\end{aligned}
$$

With a decaying $\alpha(k)$, the convergence of $\Pi_{x, 0: \bar{d}}(k)$ to 0 is proved if $\Xi_{B}$ is exponentially stable, which we shall show below.

$$
\text { Define } \bar{\Xi}_{B}=\left[\begin{array}{cccc}
1 & 0 & \cdots & 0 \\
h B & & & \\
0 & & \Xi_{B} \\
\vdots & & \\
0 & &
\end{array}\right] \text {. Note that under the condition 1) of Theorem } 7.4 \text { G is }
$$

strongly connected, or any node $i$ serves as a root of some spanning tree of $\mathcal{G}$, which also holds for $\mathcal{G}(\Xi)$ after adding virtual nodes. Furthermore, in the light of the fact that at least one agent can connect to $\mathcal{X}$, we can see that $\mathcal{G}\left(\bar{\Xi}_{B}\right)$ has a spanning tree rooted at the first node, with $\bar{\Xi}_{B}(1,1)=1>0$. 
By imposing Lemma 3.1, the other eigenvalues of $\bar{\Xi}_{B}$ than the simple unit eigenvalue fall inside the open unit disk, which proves the exponential stability of $\Xi_{B}$. The convergence to the constraint set is hence established.

3) Consensus. Denote

$$
x(k)=\left[x_{1}(k), \ldots, x_{N}(k)\right], x_{0: \bar{d}}(k)=\operatorname{col}\left\{x(k), x_{1}^{v}(k), \ldots, x_{\bar{d}}^{v}(k)\right\}
$$

where $x_{d}^{v}(k)=\left[x_{1, d}^{v}(k), \ldots, x_{N, d}^{v}(k)\right]$ for $d=1, \ldots, \bar{d}$. Without loss of generality we assume $x_{i}(k)$ to be a scalar, otherwise we use Kronecker product for the analysis. By $(7.46)$ and $(7.48)$ it is clear that

$$
x_{0: \bar{d}}(k)=\Xi x_{0: \bar{d}}(k-1)+h B_{0} \pi(k)+h \alpha(k) g(k),
$$

where $B$ and $\Xi$ are defined in 7.54 , and

$$
\begin{aligned}
B_{0} & =\operatorname{diag}(B, 0, \ldots, 0), \\
\pi(k) & =\operatorname{col}\left\{P_{C}\left(x_{1}(k)\right)-x_{1}(k), \ldots, P_{C}\left(x_{N}(k)\right)-x_{N}(k), 0, \ldots, 0\right\}, \\
g(k) & =\operatorname{col}\left\{g_{1}\left(s_{1}(k)\right), \ldots, g_{1}\left(s_{N}(k)\right), 0, \ldots, 0\right\} .
\end{aligned}
$$

Moreover, let $\Delta=I-\mathbf{1}_{N(1+\bar{d})} \mu^{\prime}$ with $\mu=c \omega$, where $\omega$ is the left eigenvector of $\Xi$ defined by

$$
\begin{aligned}
\omega & =\operatorname{col}\left\{\omega_{0}, \omega_{1}, \ldots, \omega_{\bar{d}}\right\} \\
\omega_{0} & =\mathbf{1}_{N(1+\bar{d})}, \omega_{d}=\eta \sum_{i=d}^{\bar{d}} A_{i}^{\prime} \mathbf{1}_{N}, d=1, \ldots, \bar{d}
\end{aligned}
$$

and $c \omega^{\prime} \mathbf{1}_{N(1+\bar{d})}=1$. By premultiplying $\Delta$ on both sides of 7.55 we obtain

$$
\Delta x_{0: \bar{d}}(k+1)=\Xi \Delta x_{0: \bar{d}}(k)+h \Delta\left(B_{0} \pi(k)+\alpha(k) g(k)\right)
$$

in view of $\Delta \Xi=\Xi \Delta$. Obviously, the consensus $6.2 \mathrm{a}$ is achieved once $\Delta x_{0: \bar{d}}(k) \rightarrow 0$ as $k \rightarrow \infty$.

Now let $T^{-1} \Xi T=\Lambda_{\Xi}$ be the Jordan decomposition of $\Xi$ with $T(\cdot, 1)=\mathbf{1}_{N(1+\bar{d})}$ and $T^{-1}(1, \cdot)=$ $\mu^{\prime}$. It can be inferred from Lemma 3.2 that $\Xi$ is an SIA matrix, and hence $\Lambda_{\Xi}=\operatorname{diag}\left\{1, \Lambda_{\Xi}^{1}\right\}$ with a stable $\Lambda_{\Xi}^{1}$. Now let $y(k)=T^{-1} \Delta x_{0: \bar{d}}(k)$, then $y(k)(1) \equiv 0$ with $\mu^{\prime} \Delta x=0$ for any $x$, and we denote $y(k)=\left[0, y^{1}(k)^{\prime}\right]^{\prime}$. By 7.57 we get

$$
y^{1}(k+1)=\Lambda_{\Xi}^{1} y^{1}(k)+h T_{1} \Delta\left(B_{0} \pi(k)+\alpha(k) g(k)\right),
$$

where $T_{1}$ is a submatrix of $T$ by removing the first row. The consensus is readily achieved from an exponentially stable $\Lambda_{\Xi}^{1}$, together with the decaying $\pi(k)$ and $\alpha(k)$. 
4) Optimum Set Convergence. As shown in the delay-free case, the proof is based on (7.23). In the presence of fixed time delays, we shall also establish a similar relationship; more exactly, we are to prove two inequalities similar to 7.22 and 7.23 .

Define $\Pi_{s, 0: \bar{d}}^{*}(k)$ by replacing $\mathcal{X}$ with $\mathcal{X}^{*}$ in 27.50 , which applies similarly to $\Pi_{x, 0: \bar{d}}^{*}(k)$. Furthermore, define $\chi_{s, 0: \bar{d}}(k)=\Pi_{s, 0: \bar{d}}^{*}(k)^{\prime} \Lambda_{\omega} \Pi_{s, 0: \bar{d}}^{*}(k)$ and $\chi_{x, 0: \bar{d}}(k)=\Pi_{x, 0: \bar{d}}^{*}(k)^{\prime} \Lambda_{\omega} \Pi_{x, 0: \bar{d}}^{*}(k)$, where $\Lambda_{\omega}=$ $\operatorname{diag}\{\omega\}$ with $\omega$ defined in 7.56 . Similar to 7.51 , we have

$$
\left|s_{i}(k)\right|_{\mathcal{X}^{*}} \leq\left(1-\eta D_{i}\right)\left|x_{i}(k)\right|_{\mathcal{X}^{*}}+\eta \sum_{j \in \mathcal{N}_{i}} w_{i j}\left|x_{j}\left(k-d_{i j}\right)\right|_{\mathcal{X}^{*}},
$$

and combining $\left|s_{i, d}^{v}(k)\right|_{\mathcal{X}^{*}}=\left|x_{i, d-1}^{v}(k)\right|_{\mathcal{X}^{*}}$ we arrive at

$$
\Pi_{s, 0: \bar{d}}^{*}(k) \leq \Xi \Pi_{x, 0: \bar{d}}^{*}(k),
$$

where $\Xi$ has been defined in 7.54). Therefore, by remembering $w$ as the left eigenvector of the stochastic matrix $\Xi$ with respect to 1 , and applying Lemma 3.3 it gives rise to

$$
\begin{aligned}
\chi_{s, 0: \bar{d}}(k) & \leq \Pi_{x, 0: \bar{d}}^{*}(k)^{\prime} \Xi^{\prime} \Lambda_{\omega} \Xi \Pi_{x, 0: \bar{d}}^{*}(k) \\
& \leq \Pi_{x, 0: \bar{d}}^{*}(k)^{\prime} \Lambda_{\omega} \Pi_{x, 0: \bar{d}}^{*}(k)=\chi_{x, 0: \bar{d}}(k) .
\end{aligned}
$$

By now we have proved the first inequality similar to 7.22 .

On the other hand, note that (7.8) still holds as no communication delays is involved in (7.45b). Moreover, from the set convergence and consensus results, we know that for any $\varepsilon>0$ there exists a time instant $k_{\varepsilon}$ such that

$$
\begin{array}{r}
\max _{i, d}\left\{\left|x_{i}(k)\right| \mathcal{X},\left|x_{i, d}^{v}(k)\right| \mathcal{X}\right\} \leq \varepsilon, \alpha_{k} \leq \varepsilon, \\
\max _{i, d}\left\{\left\|x_{i}(k)-\bar{x}(k)\right\|,\left\|x_{i, d}^{v}(k)-\bar{x}(k)\right\|\right\} \leq \varepsilon, \quad \forall k \geq k_{\varepsilon}
\end{array}
$$

where $\bar{x}(k)=\frac{1}{N(\bar{d}+1)} \sum_{i, d}\left(x_{i}(k)+x_{i, d}^{v}(k)\right)$. From 7.45a and 7.48a it is clear that with $\bar{s}(k)=$ $\frac{1}{N(d+1)} \sum_{i, d}\left(s_{i}(k)+x_{i, d}^{v}(k)\right)=\bar{x}(k)$ we have

$$
\begin{aligned}
\max _{i, d}\left\{\left|s_{i}(k)\right| \mathcal{X},\left|s_{i, d}^{v}(k)\right| \mathcal{X}\right\} & \leq \varepsilon, \alpha_{k} \leq \varepsilon \\
\max _{i, d}\left\{\left\|s_{i}(k)-\bar{s}(k)\right\|,\left\|s_{i, d}^{v}(k)-\bar{s}(k)\right\|\right\} & \leq \varepsilon, \forall k \geq k_{\varepsilon}+\bar{d}
\end{aligned}
$$

which rediscovers 7.21 in the presence of communication delays. Now in the light of (7.61) and 
noticing that $\omega_{0}=\mathbf{1}_{N}$, we get

$$
\begin{aligned}
\chi_{x, 0: \bar{d}}(k+1) & \leq \chi_{s, 0: \bar{d}}(k)+h \alpha(k)\left[2 \sum_{i=1}^{N}\left[f_{i}\left(P_{\mathcal{X}^{*}}(\bar{x}(k))\right)-f_{i}\left(P_{\mathcal{X}}(\bar{x}(k))\right)\right]+\varepsilon_{1}\right] \\
& =\chi_{x, 0: \bar{d}}(k)+h \alpha(k)\left[2\left(F^{*}-F\left(P_{\mathcal{X}}(\bar{x}(k))\right)\right)+\varepsilon_{1}\right], \quad \forall k \geq k_{\varepsilon}
\end{aligned}
$$

as a result of zero cost functions on the virtual nodes. The final convergence to $\mathcal{X}^{*}$ can be established similarly as in the delay-free case.

\subsection{Numerical Examples}

In this section we provide numerical examples to validate the convergence result respectively for the delay-free and fixed time delay cases. With the cost function given by $f_{i}(x)=\left\|x-x_{i}(0)\right\|$, we first discuss about the selection of the control gain $h$ in 7.4 and 7.45 .

Actually, by 6.38 we know that $\bar{\gamma}=\max _{i, g_{i}(x) \in \partial f_{i}(x)}\left\|g_{i}(x)\right\|=1$, namely that the magnitude of subgradient is independent of $x$. Furthermore, note that 7.6 holds if $f_{i}(x) \geq f_{i}(z)+\delta$. Similarly, 7.6. still holds if $\|x\| \geq\left\|x_{i}(0)\right\|+f_{i}(z)+\delta$. Therefore, by selecting $R=\max _{i}\left(\left\|x_{i}(0)\right\|+f_{i}(z)\right)+\delta$, we have

$$
\frac{2 \delta}{\bar{\gamma}^{2}+2 \bar{\gamma} R}=\frac{2 \delta}{1+2 \max _{i}\left(\left\|x_{i}(0)\right\|+f_{i}(z)\right)+2 \delta} \rightarrow 1
$$

as $\delta \rightarrow \infty$. Consequently, $h$ can be selected as $h<1$ as suggested by Remark 7.2 , which is independent of the initial values.

For the delay-free case we consider a similar example as in Section 6.5, with the communication network $\mathcal{G}$ alternates between $\mathcal{G}_{1}$ and $\mathcal{G}_{2}$ as shown in Fig. 6.1. We assume that only agent 1 can access the constraint set $\mathcal{X}=\bar{B}(0,1) \subseteq \mathbb{R}^{2}$. Moreover, take $h=0.4$ and $\eta=0.3, b=0.1$. The subgradient step size is chosen as $\alpha(k)=\frac{1}{1+k^{0.5} \ln (k+1)}$ which is neither integrable nor square integrable. We plot the plane trajectories and coordinate convergence respectively in Fig. 7.2 and 7.3 . We can see that the states of agents converge to the optimum $[0.8152,0.5792]^{\prime}$ on the unit circle.

For the fixed delay case we consider a fixed and balanced network shown in Fig. 7.4, where the weight on each edge is 1 and the only delay occurs in channel $(1,4)$ with $d_{41}=3$. The control gains and subgradient step size are selected as in the delay-free case. The plane trajectories and coordinate convergence are respectively shown in Fig. 7.5 and 7.6 . We can see that the additional fixed time delay has no effect on the final convergence. 


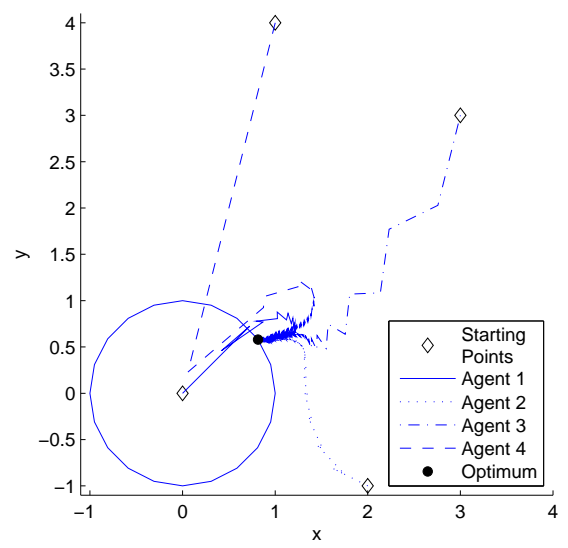

Figure 7.2: Discrete-time optimal consensus with set constraint.
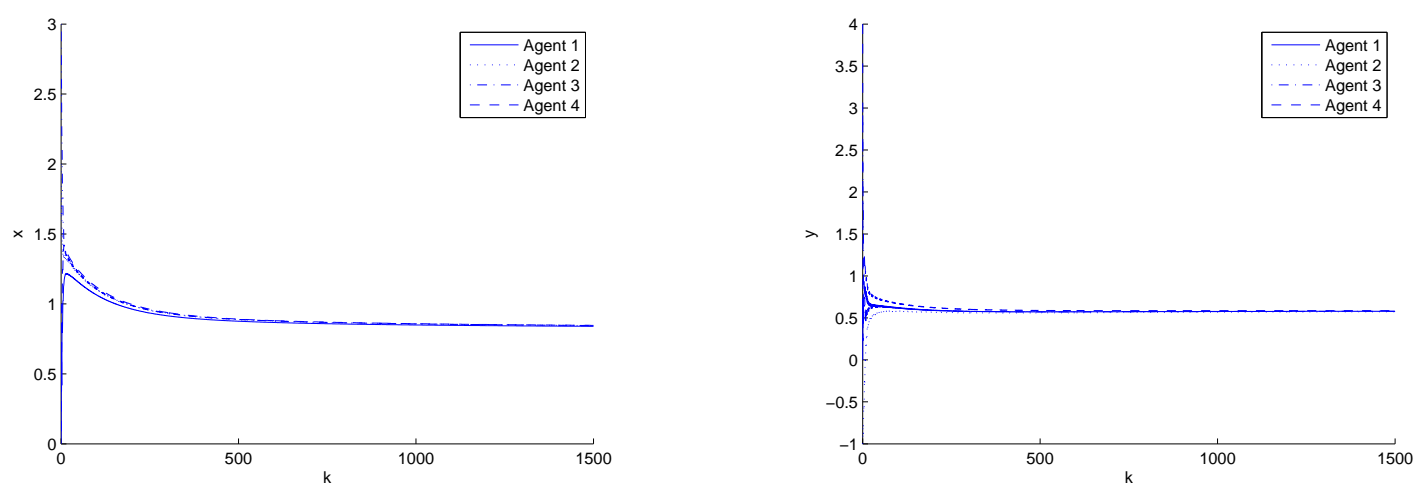

Figure 7.3: Convergence of $x, y$ coordinates.

\subsection{Conclusion}

In this chapter, we studied the discrete-time optimal consensus with a common set constraint. Similar to the continuous-time case in Chapter 6] a two-step control protocol was designed based on the local averaging, local projection, and local subgradient descent. In a similar setting, the convergence result and convergence rate analysis were rediscovered. Under a fixed network, we further showed that the necessary and sufficient conditions to guarantee the constrained optimal consensus are as follows: 1) At least one agent can access the constraint set; 2) The network is balanced and contains a spanning tree; 3) The subgradient step size is diminishing and non-summable. We also showed that the constrained optimal consensus can be still ensured in the presence of fixed time delays, simply by using delayed information in the proposed control protocol. 


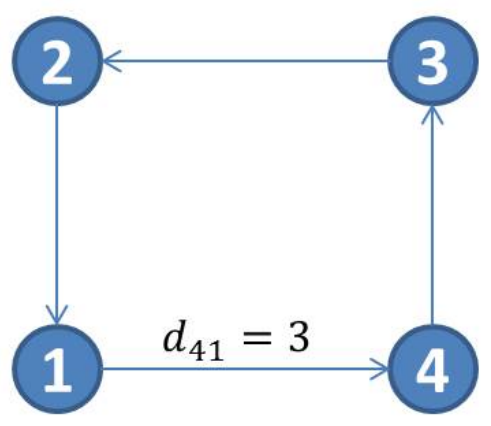

Figure 7.4: Communication graph with fixed time delays.

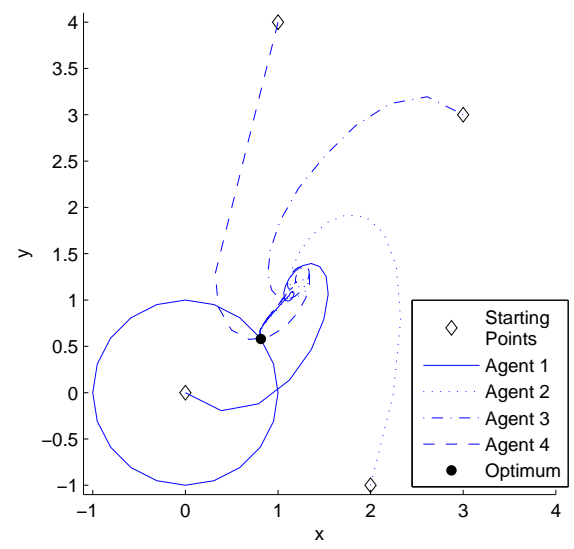

Figure 7.5: Plane trajectories of 4 agents with fixed time delays.
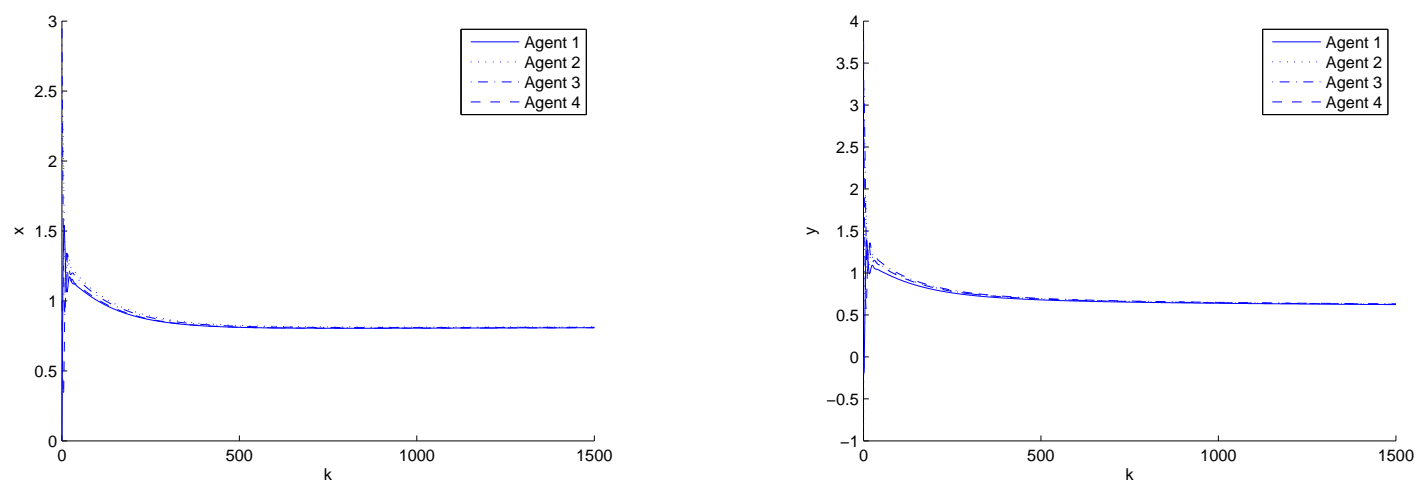

Figure 7.6: Convergence of $x, y$ coordinates with fixed time delays. 


\section{Chapter 8}

\section{Optimal Consensus of}

\section{Euler-Lagrangian Systems under}

\section{Kinematic Constraints}

In this chapter we study the optimal consensus problem of heterogeneous Euler-Lagrange (EL) systems under kinematic constraints. Under bounded velocity and control input, each agent is required to reach the minimum of the aggregate cost which is dependent on the position variable. Assuming an exact knowledge of nonlinearities in the EL equation, we first explore the case of double-integrator MASs by Lyapunov method, then extend the result to the case of EL dynamics by inverse dynamics control. To achieve the optimal consensus for double integrators, the control input is designed to consist of 4 terms: a weighted sum of position differences from its neighbors for consensus, a damping term of its velocity, a gradient descent of its individual cost for local minimization, as well as an integral feedback of the consensus error to correct the gradient differences. With properly selected control gains, the agents are able to achieve the consensus asymptotically at the minimum of the aggregate cost at an exponential rate, and finally stop at the minimum, if the network is fixed and connected. Moreover, the control gains can be tuned as sufficiently small to satisfy the bounded kinematic constraints, if an estimate of the minimum can be obtained beforehand.

The rest of this chapter is organized as follows. The problem under investigation is first formulated in Section 8.1. After establishing the optimal consensus for double-integrator MASs under kinematic constraints in Section 8.2 , we proceed to the case of EL systems in Section 8.3. A numerical example is provided in Section 8.4 and we conclude the chapter in Section 8.5 . 


\subsection{Problem Formulation}

Consider $N$ agents with the following EL dynamics:

$$
M_{i}\left(x_{i}\right) \ddot{x}_{i}+C_{i}\left(x_{i}, \dot{x}_{i}\right) \dot{x}_{i}+G_{i}\left(x_{i}\right)=\tau_{i}, i=1, \ldots, N \text {, }
$$

where $x_{i}, \dot{x}_{i}, \ddot{x}_{i} \in \mathbb{R}^{m}$ respectively denote the generalized position, velocity and acceleration vectors; $M_{i} \in \mathbb{R}^{m \times m}$ is the positive definite inertia matrix dependent on the position; $C_{i}\left(x_{i}, \dot{x}_{i}\right) \dot{x}_{i}$ is the Coriolis and centripetal forces; $G_{i}$ is the gravity, and $\tau_{i}$ is the control force. The following assumption is commonly adopted, see 129 :

Assumption 8.1. $\left\|C_{i}\left(x_{i}, \dot{x}_{i}\right)\right\| \leq C\left\|\dot{x}_{i}\right\|$ for $C \geq 0$.

Moreover, each agent $i$ is assigned with an individual cost $f_{i}$, and we aim to design a distributed control law for all agents to cooperatively minimize the aggregate cost:

$$
\min _{q \in \mathbb{R}^{m}} F(x)=\sum_{i} f_{i}(x)
$$

or equivalently

$$
\min _{x_{i} \in \mathbb{R}^{m}, i=1, \ldots, N} \sum_{i} f_{i}\left(x_{i}\right) \text {, s.t. } x_{1}=\cdots=x_{N} .
$$

Therefore, if we denote the optimum of 8.2 as $x^{*}$, then we shall prove that

$$
\lim _{t \rightarrow \infty}\left(x_{i}-x^{*}\right)=0, i=1, \ldots, N
$$

Furthermore, for practical implementation the velocity and control forces have to be bounded. Specifically, noticing that the gravity is a downward force and usually not considered in the desired movement for vehicles, say in the case of ground robots it is canceled by the normal force, and in the case of UAVs it has to be canceled by propeller thrust for floating, we let $\tilde{\tau}_{i}=\tau_{i}-G_{i}$ and define the bounded constraints as

$$
\left\|\dot{x}_{i}\right\|_{\infty} \leq \bar{V},\left\|\tilde{\tau}_{i}\right\|_{\infty} \leq \bar{U}
$$

Below we make two assumptions respectively about the communication topology and the individual cost functions.

Assumption 8.2. The communication graph $\mathcal{G}$ is connected.

Assumption 8.3. For each $i, f_{i}$ is differentiable and $\beta$-strongly convex, and its gradient $\nabla f_{i}$ is $\theta$-Lipschitz continuous, namely

$$
\left\|\nabla f_{i}(x)-\nabla f_{i}(y)\right\| \leq \theta\|x-y\|, \forall x, y .
$$


In the sequel we are to study the optimal consensus 8.3 of multiple EL systems under constraints (8.4). Noticing that any EL system with an exact knowledge of nonlinearities can be transformed into a double-integrator system, we shall study a MAS with double integrator dynamics and kinematic constraints first, and then proceed to investigate the EL case by employing the inverse dynamics control.

\subsection{Optimal Consensus of MAS with Double Integrator Dy- namics and Kinematic Constraints}

In this section we explore the optimal consensus under kinematic constraints for double-integrator MASs in the following form:

$$
\left\{\begin{array}{l}
\dot{p}_{i}=v_{i}, \\
\dot{v}_{i}=u_{i},
\end{array}\right.
$$

where $u_{i} \in \mathbb{R}^{m}$ is the control input designed as follows:

$$
\left\{\begin{array}{l}
u_{i}=-k_{p} \sum_{j \in \mathcal{N}_{i}} a_{i j}\left(p_{i}-p_{j}\right)-k_{v} v_{i}-k_{g}\left(\mu_{i}+\gamma \nabla f_{i}\left(p_{i}\right)\right), \\
\dot{\mu}_{i}=k_{\delta} \sum_{j \in \mathcal{N}_{i}} a_{i j}\left[k_{\delta p}\left(p_{i}-p_{j}\right)+\left(v_{i}-v_{j}\right)\right] .
\end{array}\right.
$$

Note that the control input is adapted from 130 and consists of the following four terms: a weighted sum of position differences from its neighbors for consensus, a damping term of its velocity, a gradient descent of its individual cost for local minimization, as well as an integral feedback $\mu_{i}$ of the consensus error to correct the gradient differences. With the additional control gains of different terms, we are able to deal with the kinematic constraints which was not considered in [130]. The optimal consensus objective and the constraints are accordingly revised as

$$
\lim _{t \rightarrow \infty}\left(p_{i}-x^{*}\right)=0, i=1, \ldots, N,
$$

and

$$
\left\|v_{i}\right\|_{\infty} \leq \bar{V},\left\|u_{i}\right\|_{\infty} \leq \bar{U}
$$

Now we present the main lemma.

Lemma 8.1. Let Assumptions 8.2 and 8.3 hold. Given the controller (8.7) with the initial condition $\mu_{i}(0)=0$ for each $i$, we can always find proper control gains $k_{v}, k_{p}, k_{g}, k_{\delta}, k_{\delta p}$ and $\gamma$ such that the optimal consensus 8.3] is achieved at an exponential rate. Moreover, if we let $v_{i}(0)=0$, and assume that initially the optimum $x^{*}$ is within the range of $R$ from each agent $\left(\left\|x^{*}-p_{i}(0)\right\| \leq R\right)$ where $R$ is 
a known constant, then the gains can be further refined to achieve the optimal consensus (8.3) under constraints 8.4].

Proof. The proof is divided into 4 parts as follows. In the first place we introduce some notations and rewrite the closed-loop system around the equilibrium, then we construct a Lyapunov function candidate for convergence analysis. After that we study how to choose proper control gains to guarantee the Lyapunov stability, and finally we refine the gains to satisfy the constraints 8.4]). Moreover, without loss of generality we let $m=1$. We also write $p^{*}=x^{*}$ for better readability.

1) Disagreement dynamics. Denote

$$
\begin{aligned}
p & =\operatorname{col}\left\{p_{1}, \ldots, p_{N}\right\}, \quad v=\operatorname{col}\left\{v_{1}, \ldots, v_{N}\right\}, \\
\mu & =\operatorname{col}\left\{\mu_{1}, \ldots, \mu_{N}\right\}, \quad u=\operatorname{col}\left\{u_{1}, \ldots, u_{N}\right\}, \\
\tilde{F}(p) & =\sum_{i} f_{i}\left(p_{i}\right), \quad P^{*}=\mathbf{1} \otimes p^{*}, \\
g^{*} & =-\nabla \tilde{F}\left(P^{*}\right)=-\operatorname{col}\left\{\nabla f_{1}\left(p^{*}\right), \ldots, \nabla f_{N}\left(p^{*}\right)\right\} .
\end{aligned}
$$

Furthermore, let $\rho=p-P^{*}$ and $\delta=\mu-\gamma g^{*}$, and combine (8.6) and 8.7) into a closed-loop system as

$$
\left\{\begin{array}{l}
\dot{\rho}=v, \\
\dot{v}=-k_{p} L \rho-k_{v} v-k_{g}\left(\delta+\gamma h\left(p, P^{*}\right)\right), \\
\dot{\delta}=k_{\delta} L\left(k_{\delta p} \rho+v\right),
\end{array}\right.
$$

where $L$ is the Laplacian matrix of the graph, and $h\left(p, P^{*}\right)=\nabla F(p)-\nabla F\left(P^{*}\right)$. Below $h\left(p, P^{*}\right)$ will be abbreviated as $h$. Under Assumption 8.2 , we can always find an orthogonal matrix $Q=[q \tilde{Q}]$ such that $Q^{\prime} L Q=\operatorname{diag}\left\{0, \lambda_{2}, \ldots, \lambda_{N}\right\}$ with $0<\lambda_{2} \leq \cdots \leq \lambda_{N}$, where $q=\frac{1}{\sqrt{N}} \mathbf{1}_{N}$. Obviously $\tilde{Q}^{\prime} L \tilde{Q}=\operatorname{diag}\left\{\lambda_{2}, \ldots, \lambda_{N}\right\} \triangleq \Lambda_{L}^{+}$. Applying the following transformations $\hat{\rho}=\operatorname{col}\left\{\hat{\rho}_{1}, \hat{\rho}_{2: N}\right\}=Q^{\prime} \rho$, $\hat{v}=Q^{\prime} v=\operatorname{col}\left\{\hat{v}_{1}, \hat{v}_{2: N}\right\}$ and $\hat{\delta}=\operatorname{col}\left\{\hat{\delta}_{1}, \hat{\delta}_{2: N}\right\}=Q^{\prime} \delta$ to the system 8.9 yields that

$$
\begin{aligned}
& \left\{\begin{array}{l}
\dot{\hat{\rho}}_{1}=\hat{v}_{1}, \\
\dot{\hat{v}}_{1}=-k_{v} \hat{v}_{1}-k_{g} \gamma q^{\prime} h, \\
\dot{\hat{\delta}}_{1}=0,
\end{array}\right. \\
& \left\{\begin{array}{l}
\dot{\hat{\rho}}_{2: N}=\hat{v}_{2: N} \\
\dot{\hat{v}}_{2: N}=-k_{p} \Lambda_{L}^{+} \hat{\rho}_{2: N}-k_{v} \hat{v}_{2: N}-k_{g} \hat{\delta}_{2: N}-k_{g} \gamma \tilde{Q}^{\prime} h, \\
\dot{\hat{\delta}}_{2: N}=k_{\delta} \Lambda_{L}^{+}\left(k_{\delta p} \hat{\rho}_{2: N}+\hat{v}_{2: N}\right),
\end{array}\right.
\end{aligned}
$$

where $\hat{\rho}_{1}, \hat{v}_{1}, \hat{\delta}_{1} \in \mathbb{R}^{m}$. Note that $8.10 \mathrm{a}$ holds on the ground of $q^{\prime} \delta=\frac{1}{\sqrt{N}} \mathbf{1}_{N}^{\prime}\left(\mu-g^{*}\right)=0$, since we have $\mathbf{1}_{N}^{\prime} g^{*}=0$ at the equilibrium $P^{*}$, and $\mathbf{1}_{N}^{\prime} \mu \equiv 0$ by observing that $\mathbf{1}_{N}^{\prime} \dot{\mu} \equiv 0$ from 8.9 together with the initial condition $\mathbf{1}_{N}^{\prime} \mu(0)=\sum_{i} \mu_{i}(0)=0$. 
2) Quadratic Lyapunov function. To prove the convergence to the optimum, we define the following two functions for constructing a quadratic Lyapunov function candidate $V=V_{1}+\sigma V_{2}$ :

$$
\begin{aligned}
& V_{1}=\frac{1}{2}\|\hat{v}\|^{2}+\varepsilon_{1} \hat{\rho}^{\prime} \hat{v}+\frac{\varepsilon_{2}}{2}\|\hat{\rho}\|^{2}+\frac{k_{g}}{2 k_{\delta}} \hat{\delta}_{2: N}^{\prime}\left(\Lambda_{L}^{+}\right)^{-1} \hat{\delta}_{2: N}, \\
& V_{2}=\frac{1}{2}\left\|\hat{v}_{2: N}+\hat{\delta}_{2: N}\right\|^{2},
\end{aligned}
$$

where $\varepsilon_{1}, \varepsilon_{2}$ and $\sigma$ are all positive constants to be determined later. For the first part, we find that

$$
\begin{aligned}
\dot{V}_{1}= & -k_{p} \hat{\rho}_{2: N}^{\prime} \Lambda_{L}^{+} \hat{v}_{2: N}-\left(k_{v}-\varepsilon_{1}\right)\|\hat{v}\|^{2} \\
& -k_{p} \varepsilon_{1} \hat{\rho}_{2: N}^{\prime} \Lambda_{L}^{+} \hat{\rho}_{2: N}-k_{g}\left(v+\gamma \varepsilon_{1} \rho\right)^{\prime} h \\
\leq & \frac{1}{2} k_{p} \lambda_{N}\left(\|\hat{\rho}\|^{2}+\|\hat{v}\|^{2}\right)-\left(k_{v}-\varepsilon_{1}\right)\|\hat{v}\|^{2} \\
& +k_{g} \gamma\left[\frac{1}{2} \theta\left(c_{1}\|v\|^{2}+\frac{1}{c_{1}}\|\rho\|^{2}\right)-\varepsilon_{1} \beta\|\rho\|^{2}\right] \\
= & -\left(k_{g} \gamma \varepsilon_{1} \beta-\frac{\theta k_{g} \gamma}{2 c_{1}}-\frac{1}{2} k_{p} \lambda_{N}\right)\|\hat{\rho}\|^{2} \\
& -\left(k_{v}-\varepsilon_{1}-\frac{1}{2} k_{p} \lambda_{N}-\frac{1}{2} \theta k_{g} \gamma c_{1}\right)\|\hat{v}\|^{2},
\end{aligned}
$$

where $c_{1}>0$ is a constant to be determined later. To obtain 8.12), we let $k_{\delta p}=\varepsilon_{1}$ and $\varepsilon_{2}=k_{v} \varepsilon_{1}$ to respectively cancel the cross term $\left\langle\hat{\rho}_{2: N}, \hat{\delta}_{2: N}\right\rangle$ and $\langle\hat{\rho}, \hat{v}\rangle$ for the first equality. In the second inequality, we used the following facts that

$$
\begin{aligned}
-\langle v, h\rangle & =-\sum_{i}\left\langle v_{i}, \nabla f_{i}\left(\rho_{i}+p^{*}\right)-\nabla f_{i}\left(p^{*}\right)\right\rangle \\
& \leq \theta \sum_{i}\left\|v_{i}\right\|\left\|\rho_{i}\right\| \leq \frac{1}{2} \theta\left(c_{1}\|v\|^{2}+\frac{1}{c_{1}}\|\rho\|^{2}\right)
\end{aligned}
$$

by recalling $\theta$ as the Lipschitz constant of $\nabla f_{i}$ 's in 8.5 , and

$$
\begin{aligned}
-\langle\rho, h\rangle & =-\sum_{i}\left\langle p_{i}-p^{*}, \nabla f_{i}\left(p_{i}\right)-\nabla f_{i}\left(p^{*}\right)\right\rangle \\
& \leq-\beta \sum_{i}\left\|p_{i}-p^{*}\right\|^{2}=-\beta\|\rho\|^{2}
\end{aligned}
$$

by recalling the $\beta$-strong convexity of $f_{i}$ 's. As for the third one, we noted that $\|\hat{\rho}\|=\|\rho\|$ and $\|\hat{v}\|=\|v\|$ by the orthogonality of $Q$. In a similar vein, we find $\dot{V}_{2}$ as

$$
\begin{aligned}
\dot{V}_{2}= & -k_{v}\left\|\hat{v}_{2: N}\right\|^{2}-k_{g}\left\|\hat{\delta}_{2: N}\right\|^{2}-\left(k_{g}+k_{v}\right) \hat{v}_{2: N}^{\prime} \hat{\delta}_{2: N} \\
& -k_{g} \gamma\left(\hat{v}_{2: N}+\hat{\delta}_{2: N}\right)^{\prime} \tilde{Q}^{\prime} h+k_{\delta} \hat{v}_{2: N}^{\prime} \Lambda_{L}^{+}\left(\hat{v}_{2: N}+\hat{\delta}_{2: N}\right) \\
& +\left(\varepsilon_{1} k_{\delta}-k_{p}\right) \hat{\rho}_{2: N}^{\prime} \Lambda_{L}^{+}\left(\hat{v}_{2: N}+\hat{\delta}_{2: N}\right) \\
\leq & \sigma_{1}\|\hat{\rho}\|^{2}+\sigma_{2}\left\|\hat{v}_{2: N}\right\|^{2}+\sigma_{3}\left\|\hat{\delta}_{2: N}\right\|^{2},
\end{aligned}
$$

where $\sigma_{1}=\frac{1}{2}\left[k_{g} \gamma \theta(1+\theta)+\left|\varepsilon_{1} k_{\delta}-k_{p}\right| \lambda_{N}\left(\frac{1}{c_{4}}+c_{5}\right)\right], \sigma_{2}=\frac{1}{2}\left[c_{2}\left(k_{g}+k_{v}\right)+k_{g} \gamma \theta+k_{\delta} \lambda_{N}\left(2+c_{3}\right)+\right.$ 
$\left.\left|\varepsilon_{1} k_{\delta}-k_{p}\right| \lambda_{N} c_{4}\right], \sigma_{3}=\frac{1}{2}\left[-k_{g}+\frac{1}{c_{2}}\left(k_{g}+k_{v}\right)+\frac{1}{c_{3}} k_{\delta} \lambda_{N}+\frac{1}{c_{5}}\left|\varepsilon_{1} k_{\delta}-k_{p}\right| \lambda_{N}\right]$, and $c_{i}>0, i=2, \ldots, 5$ are constants to be determined later. Combining 8.12 and 8.15 we obtain

$$
\begin{aligned}
\dot{V}= & \dot{V}_{1}+\sigma \dot{V}_{2} \\
\leq & -\left(k_{g} \gamma \varepsilon_{1} \beta-\frac{\theta k_{g} \gamma}{2 c_{1}}-\frac{1}{2} k_{p} \lambda_{N}-\sigma \sigma_{1}\right)\|\hat{\rho}\|^{2} \\
& -\left(k_{v}-\varepsilon_{1}-\frac{1}{2} k_{p} \lambda_{N}-\frac{1}{2} \theta k_{g} \gamma c_{1}-\sigma \sigma_{2}\right)\|\hat{v}\|^{2} \\
& -\frac{\sigma}{2}\left[k_{g}-\frac{1}{c_{2}}\left(k_{g}+k_{v}\right)-\frac{1}{c_{3}} k_{\delta} \lambda_{N}-\frac{1}{c_{5}}\left|\varepsilon_{1} k_{\delta}-k_{p}\right| \lambda_{N}\right]\left\|\hat{\delta}_{2: N}\right\|^{2} .
\end{aligned}
$$

Below we consider the selection of positive constants in 8.16 to guarantee an exponential convergence.

3) Optimal consensus. By the definition of $V_{1}$ and $V_{2}$ in 8.11) we have $V \geq 0$ if $\varepsilon_{1}^{2}<\varepsilon_{2}$ (see (8.19), which yields $k_{v}>\varepsilon_{1}$ by noting that $\varepsilon_{2}=k_{v} \varepsilon_{1}$. Furthermore, remember that an exponential convergence can be ensured by the negative definite derivative of a quadratic Lyapunov function, which is satisfied from 8.16 if the following conditions hold:

$$
\begin{aligned}
k_{g} \gamma \varepsilon_{1} \beta & >\frac{\theta k_{g} \gamma}{2 c_{1}}+\frac{1}{2} k_{p} \lambda_{N}+\sigma \sigma_{1}, \\
k_{v} & >\varepsilon_{1}+\frac{1}{2} k_{p} \lambda_{N}+\frac{1}{2} \theta k_{g} \gamma c_{1}+\sigma \sigma_{2}, \\
k_{g} & >\frac{1}{c_{2}}\left(k_{g}+k_{v}\right)+\frac{1}{c_{3}} k_{\delta} \lambda_{N}+\frac{1}{c_{5}}\left|\varepsilon_{1} k_{\delta}-k_{p}\right| \lambda_{N} .
\end{aligned}
$$

Note from the second inequality of (8.17) that $k_{v}>\varepsilon_{1}$ is automatically satisfied. To satisfy the inequalities in (8.17), we make the following observations on the right-hand side. First note that $c_{2}, c_{3}$ and $c_{5}$ can be set large enough to make the corresponding terms negligible. Furthermore, $\sigma$ can be set sufficiently small to mitigate large $\sigma_{i}$ 's which are dependent on $c_{i}, i=2, \ldots, 5$. In this light, 8.17) can be reduced to

$$
\begin{aligned}
k_{g} \gamma \varepsilon_{1} \beta & >\frac{\theta k_{g} \gamma}{2 c_{1}}+\frac{1}{2} k_{p} \lambda_{N}, \\
k_{v} & >\varepsilon_{1}+\frac{1}{2} k_{p} \lambda_{N}+\frac{1}{2} \theta k_{g} \gamma c_{1}, \\
k_{g} & >0 .
\end{aligned}
$$

Obviously $k_{p}$ can be set as sufficiently small, and we can further choose $c_{1}=\frac{\theta}{\varepsilon_{1} \omega}$ to satisfy the first inequality, and sufficiently large $k_{v}$ for the second one, while the third one holds naturally. Till now we have established the optimal consensus of the double-integrator MASs 8.6 by choosing proper control gains.

4) Optimal consensus with kinematic constraints. In this part we proceed to refine the control 
gains to satisfy the bounded constraints. Noticing that

$$
\begin{aligned}
\|u\| & =\left\|-k_{p} L \rho-k_{v} v-k_{g}(\delta-\gamma h)\right\| \\
& \leq k_{p} \lambda_{N}\|\rho\|+k_{v}\|v\|+k_{g}(\|\delta\|+\gamma \sqrt{N} \theta\|\rho\|),
\end{aligned}
$$

we have to find the corresponding estimates of each component and then design the control gains to satisfy the given constraint.

First of all, once 8.17 is satisfied, we have $V_{1}(t) \leq V(0)$ for $t \geq 0$ due to $\dot{V} \leq 0$. It can be seen that

$$
\begin{aligned}
& \frac{1}{2}\|\hat{v}\|^{2}+\varepsilon_{1} \hat{\rho}^{\prime} \hat{v}+\frac{\varepsilon_{2}}{2}\|\hat{\rho}\|^{2} \\
= & \frac{1}{2}\left(v+\varepsilon_{1} \rho\right)^{2}+\frac{1}{2}\left(\varepsilon_{2}-\varepsilon_{1}^{2}\right)\|\rho\|^{2} \\
= & \frac{\varepsilon_{2}}{2}\left(\rho+\frac{\varepsilon_{1}}{\varepsilon_{2}} v\right)^{2}+\frac{1}{2}\left(1-\frac{\varepsilon_{1}^{2}}{\varepsilon_{2}}\right)\|v\|^{2},
\end{aligned}
$$

and it holds with $b_{1}>0$ and $v_{0}=0$ that

$$
\frac{k_{g}}{2 k_{\delta} \lambda_{N}}\|\delta\|^{2} \leq V(0) \leq \frac{1}{2}\left(b_{1} \varepsilon_{1}+\varepsilon_{2}\right)\left\|\rho_{0}\right\|^{2}+\left[\sigma+\frac{k_{g}}{2 k_{\delta} \lambda_{2}}\right]\left\|\delta_{0}\right\|^{2},
$$

where the subscript 0 for $\rho, v$, and $\delta$ denotes the corresponding initial values. Given $\delta_{0}=\mu_{0}-\gamma g^{*}=$ $-\gamma g^{*}$, we further find from 8.19 and 8.20 that

$$
\begin{aligned}
\|\rho\|^{2} & \leq \frac{\varepsilon_{1}^{2}+\varepsilon_{2}}{\varepsilon_{2}-\varepsilon_{1}^{2}}\left\|\rho_{0}\right\|^{2}+\frac{2 \sigma+\frac{k_{g}}{k_{\delta} \lambda_{2}}}{\varepsilon_{2}-\varepsilon_{1}^{2}}\left\|\gamma g^{*}\right\|^{2}, \\
\|v\|^{2} & \leq \frac{\varepsilon_{1}^{2}+\varepsilon_{2}}{1-\varepsilon_{1}^{2} / \varepsilon_{2}}\left\|\rho_{0}\right\|^{2}+\frac{2 \sigma+\frac{k_{g}}{k_{\delta} \lambda_{2}}}{1-\varepsilon_{1}^{2} / \varepsilon_{2}}\left\|\gamma g^{*}\right\|^{2}, \\
\|\delta\|^{2} & \leq \frac{k_{\delta}\left(\varepsilon_{1}^{2}+\varepsilon_{2}\right) \lambda_{N}}{k_{g}}\left\|\rho_{0}\right\|^{2}+\left(\frac{2 \lambda_{N} k_{\delta} \sigma}{k_{g}}+\frac{\lambda_{N}}{\lambda_{2}}\right)\left\|\gamma g^{*}\right\|^{2},
\end{aligned}
$$

if we let $b_{1}=\varepsilon_{1}$. Moreover, by letting $\varepsilon_{1}=\varepsilon^{\frac{1}{2}}, k_{v}=\kappa_{v} \varepsilon^{\frac{1}{2}}$ with $\kappa_{v}>1, k_{g}=\varepsilon$ and $k_{\delta}=\varepsilon^{\alpha}$ with $\alpha<1$, we get $\varepsilon_{2}=k_{v} \varepsilon_{1}=\kappa_{v} \varepsilon$ and simplify (8.21) as

$$
\begin{aligned}
& \|\rho\|^{2} \leq \frac{\kappa_{v}+1}{\kappa_{v}-1}\left\|\rho_{0}\right\|^{2}+\frac{2 \sigma+\varepsilon^{1-\alpha} / \lambda_{2}}{\left(\kappa_{v}-1\right) \varepsilon}\left\|\gamma g^{*}\right\|^{2}, \\
& \|v\|^{2} \leq \kappa_{v} \varepsilon \frac{\kappa_{v}+1}{\kappa_{v}-1}\left\|\rho_{0}\right\|^{2}+\frac{2 \sigma+\varepsilon^{1-\alpha} / \lambda_{2}}{1-1 / \kappa_{v}}\left\|\gamma g^{*}\right\|^{2}, \\
& \|\delta\|^{2} \leq \varepsilon^{\alpha}\left(\kappa_{v}+1\right) \lambda_{N}\left\|\rho_{0}\right\|^{2}+\left[\frac{2 \lambda_{N} \sigma}{\varepsilon^{1-\alpha}}+\frac{\lambda_{N}}{\lambda_{2}}\right]\left\|\gamma g^{*}\right\|^{2} .
\end{aligned}
$$

Now replace $\varepsilon_{1}, k_{v}, k_{g}$ and $k_{\delta}$ in 8.17 by the corresponding quantities, and let $c_{1}=\frac{\theta}{\beta} \varepsilon^{-\frac{1}{2}}, c_{2}=$ 
$2 \kappa_{v} \varepsilon^{-\frac{1}{2}}$ and $c_{3}=c_{5}=\frac{6 \lambda_{N}}{\varepsilon^{1-\alpha}} ; 8.17$ is revised as

$$
\begin{aligned}
\gamma \beta \varepsilon^{3 / 2} & >\frac{\gamma \beta}{2} \varepsilon^{3 / 2}+\frac{1}{2} k_{p} \lambda_{N}+\sigma \sigma_{1} \\
\kappa_{v} \varepsilon^{\frac{1}{2}} & >\varepsilon^{\frac{1}{2}}+\frac{1}{2} k_{p} \lambda_{N}+\frac{\gamma}{2} \frac{\theta^{2}}{\beta} \varepsilon^{\frac{1}{2}}+\sigma \sigma_{2} \\
\varepsilon & >\frac{1}{2} \varepsilon+\frac{1}{2 \kappa_{v}} \varepsilon^{3 / 2}+\frac{1}{3} \varepsilon
\end{aligned}
$$

Letting $c_{4}=1$ and $k_{p}=o\left(\varepsilon^{\frac{3}{2}}\right)$ in 8.15 , we obtain

$$
\begin{aligned}
\sigma_{1} & =\frac{1}{2}\left[\varepsilon \gamma \theta(1+\theta)+\left|\varepsilon^{\alpha+\frac{1}{2}}-o\left(\varepsilon^{\frac{3}{2}}\right)\right| \lambda_{N}\left(1+\frac{6 \lambda_{N}}{\varepsilon^{1-\alpha}}\right)\right]=O\left(\varepsilon^{\min \left\{1,2 \alpha-\frac{1}{2}\right\}}\right), \\
\sigma_{2} & =\kappa_{v}\left(\varepsilon^{\frac{1}{2}}+\kappa_{v}\right)+\frac{\theta}{2} \gamma \varepsilon+\varepsilon^{\alpha} \lambda_{N}\left(1+\frac{3 \lambda_{N}}{\varepsilon^{1-\alpha}}\right)+\frac{\lambda_{N}}{2}\left|\varepsilon^{\alpha+\frac{1}{2}}-o\left(\varepsilon^{\frac{3}{2}}\right)\right|=O\left(\varepsilon^{\min \left\{\frac{1}{2}, 2 \alpha-\frac{1}{2}\right\}}\right),
\end{aligned}
$$

and it is readily seen from the above that we can select $\sigma=o\left(\varepsilon^{\max \left\{\frac{1}{2}, 2-2 \alpha\right\}}\right)$ to guarantee 8.23$)$.

Below we employ 8.22 to find appropriate gains to satisfy the velocity and acceleration constraints 8.4]. From the initial estimate $\left\|x^{*}-p_{i}(0)\right\| \leq R$ we get $\left\|\rho_{0}\right\| \leq \sqrt{N} R$, which follows from Assumption 8.3 that $\left\|g^{*}\right\| \leq \sqrt{N}\left[\max _{i}\left\|\nabla f_{i}\left(p_{i}(0)\right)\right\|+\theta R\right]$. Now the velocity constraint can be satisfied by imposing the second inequality in 8.22 with sufficiently small $\varepsilon$. In light of 8.17 and 8.22 we arrive at

$$
\begin{aligned}
& k_{p}\|\rho\| \leq \sqrt{\frac{\kappa_{v}+1}{\kappa_{v}-1}} o\left(\varepsilon^{\frac{3}{2}}\right)\left\|\rho_{0}\right\|+\gamma \sqrt{\frac{2 \sigma+\varepsilon^{1-\alpha} / \lambda_{2}}{\kappa_{v}-1}} o(\varepsilon)\left\|g^{*}\right\|, \\
& k_{v}\|v\| \leq \kappa_{v}^{\frac{3}{2}}\left[\sqrt{\frac{\kappa_{v}+1}{\kappa_{v}-1}} \varepsilon\left\|\rho_{0}\right\|+\gamma \sqrt{\frac{2 \sigma \varepsilon+\varepsilon^{2-\alpha} / \lambda_{2}}{\kappa_{v}-1}}\left\|g^{*}\right\|\right] \\
& k_{g}\|\delta\| \leq \sqrt{\left(\kappa_{v}+1\right) \lambda_{N} \varepsilon^{2+\alpha}}\left\|\rho_{0}\right\|+\gamma\left(\sqrt{2 \sigma \varepsilon^{1+\alpha}}+\sqrt{\frac{\lambda_{N}}{\lambda_{2}}} \varepsilon\right)\left\|g^{*}\right\|, \\
& k_{g}\|\rho\| \leq \sqrt{\frac{\kappa_{v}+1}{\kappa_{v}-1}} \varepsilon\left\|\rho_{0}\right\|+\gamma \sqrt{\frac{2 \sigma \varepsilon+\varepsilon^{2-\alpha} / \lambda_{2}}{\kappa_{v}-1}}\left\|g^{*}\right\| .
\end{aligned}
$$

Therefore, if we take $-2<\alpha<1$, then all the coefficients of $\left\|\rho_{0}\right\|$ and $\left\|g^{*}\right\|$ in 8.25 assume positive powers of $\varepsilon$. In consequence, $\varepsilon$ can be set as sufficiently small to satisfy the constraint on $u$, which completes the proof.

Remark 8.1. To prove the above result, we construct a quadratic Lyapunov function $V$ for proving the convergence of the state $\left(p_{i}, v_{i}, \mu_{i}\right)$ to the equilibrium $\left(x^{*}, 0,-\gamma \nabla f_{i}\left(x^{*}\right)\right)$. Specifically, $V$ is the sum of $V_{1}$ and $\sigma V_{2}$. If we choose $V=V_{1}$ as the Lyapunov function candidate in [130], then with $\dot{V}_{1} \leq 0$ we are ready to show the asymptotic convergence by LaSalle's invariance principle. With the additional component $V_{2}$, we can find a negative term of $\left\|\hat{\delta}_{2: N}\right\|^{2}$ which ensures the exponential stability together with negative terms of $\|\hat{\rho}\|^{2}$ and $\|\hat{v}\|^{2}$ in $V_{1}$. The introduction of a small $\sigma$ is to alleviate the positive terms of $\|\hat{\rho}\|^{2}$ and $\|\hat{v}\|^{2}$ in $\dot{V}_{2}$. Once the convergence is established, we are able to 
estimate the magnitude of each component which is dependent on the initial values, or more precisely on the knowledge of $R$ as revealed in (8.21). Furthermore, the control gains of corresponding terms can be selected as sufficiently small, and hence the bounds of velocity and acceleration can always be satisfied.

\subsection{Optimal Consensus of MAS with EL Dynamics and Kine- matic Constraints}

Assuming that $M_{i}, C_{i}$ and $G_{i}$ can be accurately obtained, we are to solve the optimal consensus problem for EL dynamical systems under constraints (8.4) by adopting inverse dynamics control. Let $\tau_{i}$ be given by

$$
\tau_{i}=M_{i}\left(x_{i}\right) u_{i}+C_{i}\left(x_{i}, \dot{x}_{i}\right) \dot{x}_{i}+G_{i}\left(x_{i}\right)
$$

with $u_{i}$ designed by 8.7 . Clearly now the original EL system 8.1) reduces to the double-integrator system as $\ddot{x}_{i}=u_{i}$, and we have $x_{i} \equiv p_{i}$ and $\dot{x}_{i} \equiv v_{i}$ if the initial values of the two systems coincide. Below we consider the selection of control gains in (8.7) to achieve the optimal consensus 8.3 under kinematic constraints 8.4.

Actually, as seen from the step 4) in the proof of Lemma 8.1. we can let $k_{p}=\varepsilon^{2}, k_{v}=\kappa_{v} \varepsilon^{\frac{1}{2}}$ with $\kappa_{v}>1+\frac{\gamma}{2} \frac{\theta^{2}}{\beta}, k_{g}=\varepsilon$ and $k_{\delta p}=\varepsilon^{\frac{1}{2}}$. By replacing $\sigma=\varepsilon^{\frac{5}{2}}, \alpha=0$ and other corresponding terms of $\varepsilon$ in 8.23 , we know that the optimal consensus 8.3 can be achieved once $\varepsilon$ satisfies the following inequalities:

$$
\begin{aligned}
\lambda_{N}\left(2+6 \lambda_{N}\right) \varepsilon^{\frac{1}{2}}+\gamma \theta(1+\theta) \varepsilon^{2} & <\beta \\
\varepsilon^{\frac{3}{2}}\left[\lambda_{N}\left(\frac{1}{2}+3 \lambda_{N}\right)+\left(\kappa_{v}^{2}+\frac{3}{2} \lambda_{N}\right) \varepsilon+\kappa_{v} \varepsilon^{\frac{3}{2}}+\frac{\gamma \theta}{2} \varepsilon^{2}\right] & <\left(\kappa_{v}-1-\frac{1}{2} \frac{\gamma \theta^{2}}{\beta}\right) \\
\varepsilon^{\frac{1}{2}} & <\min \left\{\frac{1}{3} \kappa_{v}, 1\right\} .
\end{aligned}
$$

Now denote $\bar{\rho}_{0}=\sqrt{N} R, \bar{g}=\sqrt{N}\left[\max _{i}\left\|\nabla f_{i}\left(x_{i}(0)\right)\right\|+\theta R\right]$. For the velocity constraint $\left\|\dot{x}_{i}\right\|_{\infty} \leq$ $\bar{V}$, we infer from 8.22 that it can be satisfied if the following inequality holds

$$
\frac{\kappa_{v}}{\kappa_{v}-1}\left[\left(\left(\kappa_{v}+1\right) \bar{\rho}_{0}^{2}+\frac{\gamma^{2}}{\lambda_{2}} \bar{g}^{2}\right) \varepsilon+2 \gamma^{2} \bar{g}^{2} \varepsilon^{\frac{5}{2}}\right] \leq \bar{V}^{2}
$$

For the constraint on control input, by noticing that $\tilde{\tau}_{i}=M_{i}\left(x_{i}\right) u_{i}+C_{i}\left(x_{i}, \dot{x}_{i}\right) \dot{x}_{i}$ and combining (8.18) and 8.25 we know that it can be satisfied when the following inequality is satisfied:

$$
e_{2} \varepsilon+e_{3} \varepsilon^{\frac{3}{2}}+e_{4} \varepsilon^{2}+e_{5} \varepsilon^{\frac{5}{2}} \leq \bar{U}
$$


where

$$
\begin{aligned}
e_{2}= & M \bar{\rho}_{0}\left[\sqrt{\frac{\kappa_{v}+1}{\kappa_{v}-1}}\left(\kappa_{v}^{\frac{3}{2}}+\gamma \sqrt{N} \theta\right)+\sqrt{\left(\kappa_{v}+1\right) \lambda_{N}}\right] \\
& +M \gamma \bar{g}\left[\sqrt{\frac{1}{\lambda_{2}\left(\kappa_{v}-1\right)}}(1+\gamma \sqrt{N} \theta)+\sqrt{\frac{\lambda_{N}}{\lambda_{2}}}\right]+\frac{C \kappa_{v}}{\kappa_{v}-1}\left[\left(\kappa_{v}+1\right) \bar{\rho}_{0}^{2}+\frac{\gamma^{2}}{\lambda_{2}} \bar{g}^{2}\right], \\
e_{3}= & M \gamma \bar{g} \sqrt{2} \bar{\varepsilon}^{1 / 4}\left[1+\sqrt{\frac{1}{\kappa_{v}-1}}(1+\gamma \sqrt{N} \theta)\right] \\
e_{4}= & M \gamma \bar{g} \lambda_{N} \sqrt{\frac{1}{\lambda_{2}\left(\kappa_{v}-1\right)}}, \\
e_{5}= & \frac{2 C \kappa_{v}}{\kappa_{v}-1} \gamma^{2} \bar{g}^{2}+M \gamma \bar{g} \lambda_{N} \sqrt{\frac{2 \bar{\varepsilon}^{1 / 2}}{\kappa_{v}-1}}
\end{aligned}
$$

and $M=\sup _{\left\|q-x^{*}\right\| \leq \bar{R}} \max _{i}\left\|M_{i}(q)\right\|$ with $\bar{R}=\sqrt{\frac{\kappa_{v}+1}{\kappa_{v}-1}} \bar{\rho}_{0}+\gamma \sqrt{\frac{1+\lambda_{N}}{\kappa_{v}-1}} \bar{g}$, and $\bar{\varepsilon}=\sup \{\varepsilon>0$ : $\varepsilon$ solves 8.27 and 8.28$\}$.

We summarize the above discussion in the following theorem.

Theorem 8.1. Let Assumptions 8.1, 8.2 and 8.3 hold. Consider the MAS with EL dynamics 8.1, where the control law $\tau_{i}$ is designed as 8.26) with $u_{i}$ given by (8.7). Furthermore, select $\gamma>0, k_{p}=\varepsilon^{2}$, $k_{v}=\kappa_{v} \varepsilon^{\frac{1}{2}}$ with $\kappa_{v}>1+\frac{1}{2} \frac{\theta^{2}}{\beta}, k_{g}=\varepsilon, k_{\delta}=1$ and $k_{\delta p}=\varepsilon^{\frac{1}{2}}$ in 8.26 , and set $\dot{x}_{i}(0)=\mu_{i}(0)=0$ for each agent $i$. Provided that initially the optimum $x^{*}$ is within the range of $R$ from each agent $\left(\left\|x^{*}-x_{i}(0)\right\| \leq R\right)$, where $R$ is a known constant, the optimal consensus (8.3) can be achieved under kinematic constraints (8.4) at an exponential rate, if $\varepsilon$ satisfies the inequalities 8.27), (8.28) and 8.29 .

Remark 8.2. The choice of control gains in the above is dependent on the specific Lyapunov candidate $V$. In our case, from the proof we know that after selecting $k_{\delta p}$ to simplify $\dot{V}$, it only needs a sufficiently large damping gain $k_{v}$ to guarantee an exponential convergence as implied by (8.17]). The other control gains are selected to satisfy the constraints, and for simplicity we require them to take the form of power terms of $\varepsilon$, so the problem is reduced to find the proper $\varepsilon$. Also note that 8.27 translates into 8.23 for a negative definite derivative of the selected Lyapunov candidate, while 8.28 and 8.29 enforce the corresponding constraints. Furthermore, it should be pointed out that the selection of control gains may be much conservative as shown by the example in Section 8.4. where $\bar{U}$ and $\bar{V}$ are at least two orders of magnitude larger than the maximum velocity and acceleration.

Remark 8.3. For the quadratic Lyapunov candidate in our case, we know from (8.11) and (8.19) that $V \leq \frac{1}{2}\left(\varepsilon_{2}-\varepsilon_{1}^{2}\right)\|\hat{\rho}\|^{2}+\left[\frac{1}{2}\left(1-\frac{\varepsilon_{1}^{2}}{\varepsilon_{2}}\right)+\sigma\right]\|\hat{v}\|^{2}+\left[\sigma+\frac{k_{g}}{2 k_{\delta} \lambda_{2}}\right]\left\|\hat{\delta}_{2: N}\right\|^{2}$. On the other hand, its derivative satisfies $\dot{V} \leq-c_{\rho}\|\hat{\rho}\|^{2}-c_{v}\|\hat{v}\|^{2}-c_{\delta}\left\|\hat{\delta}_{2: N}\right\|^{2}$, where $c_{\rho}, c_{v}$ and $c_{\delta}$ respectively correspond to the coefficients of $\|\hat{\rho}\|^{2},\|\hat{v}\|^{2}$ and $\|\hat{\delta}\|^{2}$ in (8.16). Therefore, given the condition (8.17), we can find the exponential convergence rate as $r=\frac{r_{1}}{r_{2}}$, where $r_{1}=\min \left\{c_{\rho}, c_{v}, c_{\delta}\right\}$ and $r_{2}=\max \left\{\frac{1}{2}\left(\varepsilon_{2}-\varepsilon_{1}^{2}\right),\left[\frac{1}{2}\left(1-\frac{\varepsilon_{1}^{2}}{\varepsilon_{2}}\right)+\sigma\right],[\sigma+\right.$ 
$\left.\left.\frac{k_{g}}{2 k_{\delta} \lambda_{2}}\right]\right\}$. If we select the control gains as in Theorem 8.1, then we get $r_{1}=\min \left\{\frac{1}{2} \gamma \beta \varepsilon^{\frac{3}{2}}-\frac{\lambda_{N}}{2} \varepsilon^{2}-\right.$ $\left.O\left(\varepsilon^{2}\right),\left(\kappa_{v}-1-\frac{1}{2} \frac{\gamma \theta^{2}}{\beta}\right) \varepsilon^{\frac{1}{2}}-O\left(\varepsilon^{2}\right),\left(\frac{1}{6}-\frac{1}{2 \kappa} \varepsilon^{\frac{1}{2}}\right) \varepsilon\right\}=O\left(\varepsilon^{\frac{3}{2}}\right), r_{2}=\max \left\{\frac{1}{2}\left(\kappa_{v}-1\right) \varepsilon, \frac{\kappa_{v}-1}{2 \kappa}+\varepsilon^{\frac{5}{2}}, \frac{1}{2 \lambda_{2}} \varepsilon+\varepsilon^{\frac{5}{2}}\right\}=$ $O(1)$. As a result, we can get an estimate of the exponential convergence rate as $r=O\left(\varepsilon^{\frac{3}{2}}\right)$. Noticing from 8.28 and 8.29 that a larger upper bound of $\bar{V}$ or $\bar{U}$ allows for a larger $\varepsilon$, we may expect a faster convergence rate in the case of a larger $\bar{V}$ or $\bar{U}$. However, it should be mentioned that this observation is only effective on the premise of 8.27 .

Remark 8.4. In general, it is difficult to obtain an a priori estimate of the optimum, especially in some special cases when the optimum is dependent on the coefficients of each individual cost function, such as the quadratic cost shown in the next section. In this case, the optimum can be estimated by exchanging the coefficients within the network. On the other hand, it is also impractical to assume an exact knowledge of nonlinearity for EL dynamics. Both issues will be addressed in future work.

\subsection{Numerical Examples}

We consider a 4-agent system with double-integrator dynamics 8.6. Assume that the 4 agents are connected as a circle with the weight given by 0.1 on each edge, and start respectively from initial positions $[0,0]^{\prime},[2,-1]^{\prime},[3,3]^{\prime}$ and $[1,4]^{\prime}$. The constraints in 8.4 are given by $\bar{V}=\bar{U}=$ 10 , respectively corresponding to the maximum velocity and acceleration of $10 \mathrm{~m} / \mathrm{s}$ and $10 \mathrm{~m} / \mathrm{s}^{2}$. Furthermore, each individual cost is given by $f_{i}=a_{i}\left(x_{i}-x_{i}(0)\right)^{2}+b_{i}\left(y_{i}-y_{i}(0)\right)^{2}$, where $\left[x_{i}, y_{i}\right]^{\prime}$ is the position of agent $i$, and the coefficients are given by $a_{i}=1,2,3,2$ and $b_{i}=2,2,1,1$. From the definition of strongly convex function we get $\beta=\min _{i}\left\{a_{i}, b_{i}\right\}=1$, and $\theta=2 \max _{i}\left\{a_{i}, b_{i}\right\}=6$. Take $\gamma=0.3$. Moreover, denoting the optimum of 8.2 as $\left(x^{*}, y^{*}\right)$, it is easily seen that $x^{*} \in\left[\min _{i} x_{i}(0), \max _{i} x_{i}(0)\right]$ and $y^{*} \in\left[\min _{i} y_{i}(0), \max _{i} y_{i}(0)\right]$. Hence, we can find that $\bar{\rho}_{0}=10.8$ and $\bar{g}=\gamma \theta \bar{\rho}_{0}=19.44$. To obtain $\varepsilon$, we set $\kappa_{v}=1+\frac{\gamma}{2} \frac{\theta^{2}}{\beta}+\frac{1}{2}=6.9$, and the solution set of inequalities (8.27), (8.28), and (8.29) with $C=0, M=1$ can be found as $(0,0.0243)$. Let $\varepsilon=0.0242$ and get $k_{p}=0.0038, k_{v}=1.0750$, $k_{g}=0.0242$ and $k_{\delta p}=0.1558$. As can be seen from Fig. 8.1, the 4 agents converge to the optimum $[1.88,0.71]^{\prime}$, while satisfying the velocity and acceleration constraints as shown respectively in Fig. 8.2 and 8.3 .

Now we increase $\varepsilon$ to 0.9 and redo the simulation. As can be seen from Fig. 8.4 , the optimal convergence can be achieved with a faster rate, and the velocity and acceleration constraints are still satisfied as respectively shown in Fig. 8.5 and 8.6. Our future study will be focused on overcoming the conservativeness in choosing the control gains. 

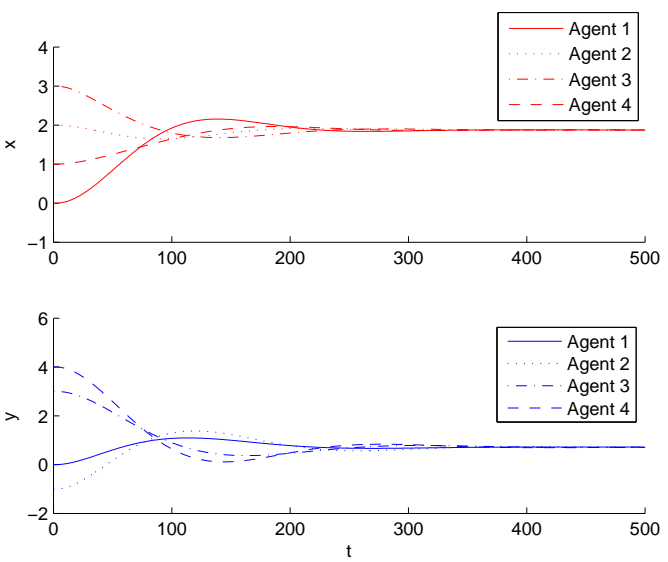

Figure 8.1: Optimal consensus of positions with small gains.
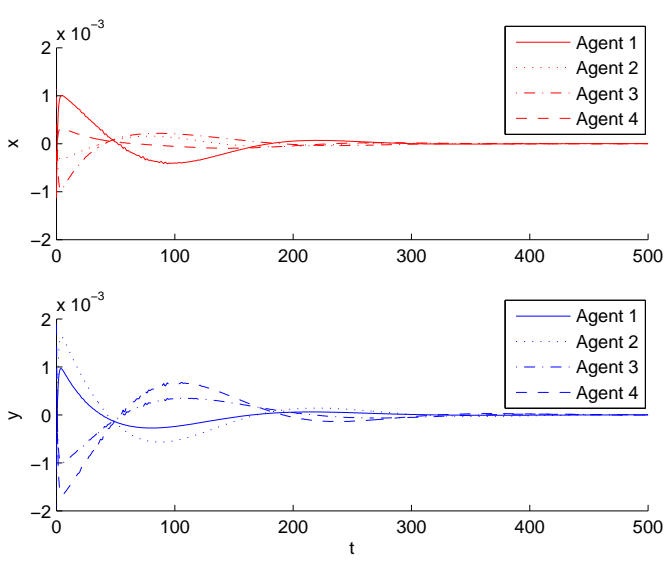

Figure 8.2: Variation of velocities with small gains.
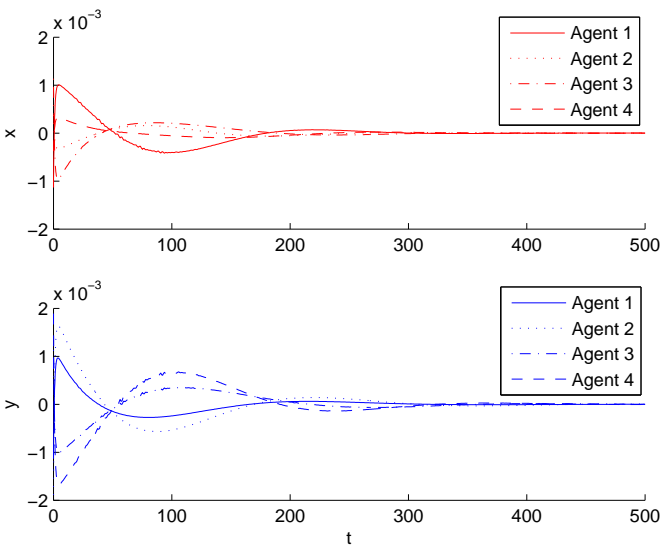

Figure 8.3: Variation of accelerations with small gains. 

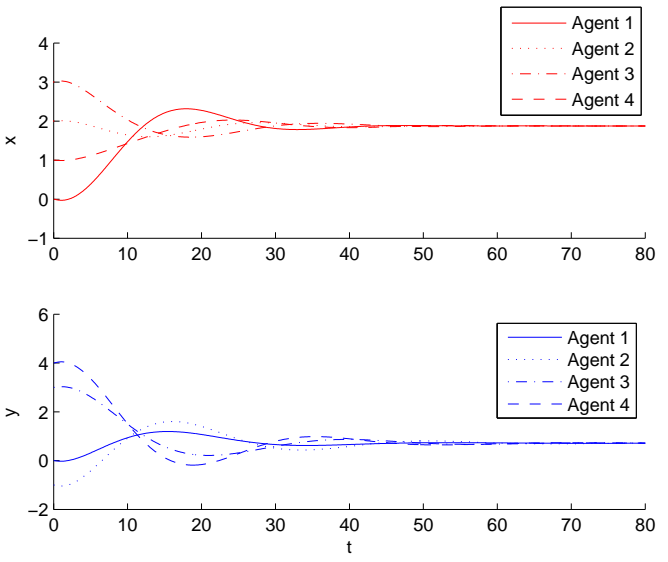

Figure 8.4: Optimal consensus of positions with larger gains.
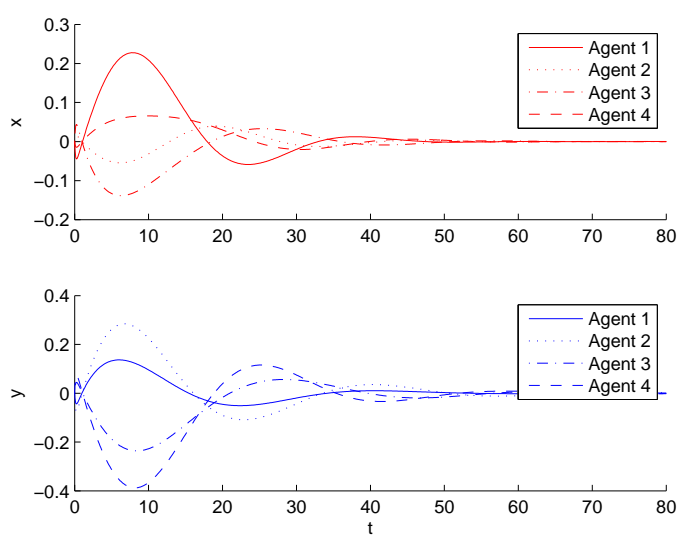

Figure 8.5: Variation of velocities with larger gains
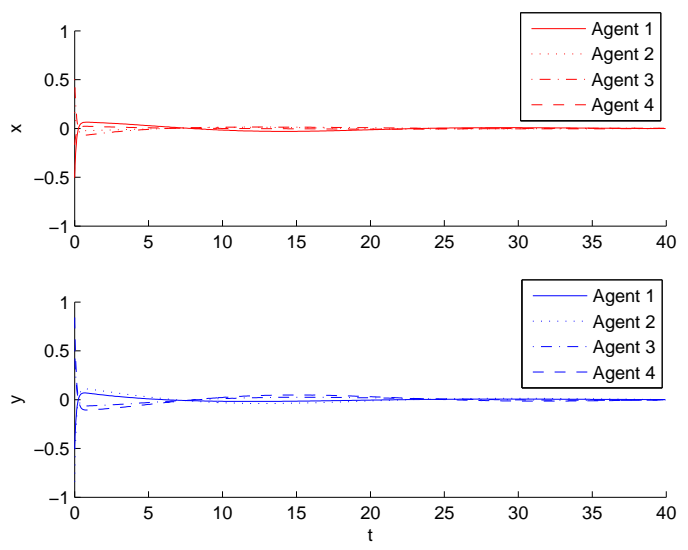

Figure 8.6: Variation of accelerations with larger gains. 


\subsection{Conclusion}

In this chapter the distributed optimal consensus problem of MASs with EL dynamics under bounded velocity and control input constraints was studied. The case of double-integrator MASs was firstly explored, where the control input of each agent is formed by a weighted average term for position consensus, a damping term of its velocity, a gradient descent of its individual cost, and an integral feedback of the consensus error to correct the gradient difference. With the aid of a quadratic Lyapunov function, it was shown that an exponentially fast convergence to the global optimum can be guaranteed under a fixed and connected topology. Furthermore, with an estimate of the initial range of the optimum, the control gains can be properly chosen to satisfy bounded kinematic constraints. The case of EL dynamics was then addressed by the inverse dynamics control, assuming that the nonlinearities can be exactly known. 


\section{Chapter 9}

\section{Conclusion and Future Work}

\subsection{Conclusion}

The recent decade has witnessed an increasing research interest in distributed consensus of MASs, with a variety of protocols developed to achieve the consensus in different scenarios. Practical constraints have to be taken into account for the implementation of these consensus protocols, and this thesis delivers an exploration in this direction. Specifically, we have considered two special kinds of consensus problem under constraints: quantized consensus under data rate constraint, and optimal consensus under set or kinematic constraints. We summarize our contributions as follows:

1. We considered the quantized consensus problem of MASs with $n$-th order integrator dynamics where only the first state variable is measurable, and provided a sufficient data rate to achieve the asymptotic consensus. A quantized-observer based encoding/decoding scheme was proposed to obtain an estimate of the unmeasurable state, which was also used in the control input. By employing perturbation techniques, we gave an accurate characterization of the influence of control input on the convergence rate, and showed that it only needs $n$ bits of information exchange between agents to achieve an exponentially fast consensus in a fixed and connected network. The analysis method is completely different from existing works, and the result extends the current results of MASs with single and double integrator dynamics.

2. We considered the quantized consensus problem of $2 m$-th order oscillator systems, and provided a sufficient data rate to achieve the asymptotic consensus. The state matrix of each agent is a $2 m$-th order real Jordan block admitting $m$ identical pairs of conjugate poles on the unit circle. Each agent has a single input, and only the first state variable can be measured. Based on the constructability of each individual system, a novel quantized-observer based encoding/decoding scheme was proposed. We studied the harmonic oscillator case $(m=1)$ in a directed network with a spanning 
tree, and the more general case $(m \geq 2)$ in an undirected and connected network. By employing perturbation and combinatoric techniques, we showed that the number of sufficient data bits to guarantee the consensus is an integer between $m$ to $2 m$, depending on the frequency of the oscillator. Notably, this result and the previous result indicate that the sufficient data rate for quantized consensus of MASs with critical high-order dynamics is only dependent on the system structure, while independent on the network topology.

3. We considered the optimal consensus problem for MASs with single integrator dynamics and a common set constraint, both in continuous-time and discrete-time systems. The control protocol consists of three terms: a weighted averaging, a projection direction towards the constraint set, and a subgradient descent with diminishing step sizes. Under mild connectivity conditions we showed that the consensus can be achieved at the constrained minimum, if the step size satisfies a persistence condition. Moreover, for strongly convex costs, we provide a systematic convergence rate analysis for different step sizes, and chose the best step size accordingly for a fastest convergence. For discrete-time MAS under a fixed network, we further discussed the necessary and sufficient conditions to ensure the optimal consensus, as well as the convergence under fixed communication delays.

4. We considered the optimal consensus problem for multiple heterogeneous EL systems under bounded kinematic constraints. We first studied the case of double integrators with the help of a quadratic Lyapunov function, and then extended the result to the EL systems by inverse dynamics control. With an initial estimate about the optimum, we showed that an exponentially fast optimal consensus can be achieved under bounded kinematic constraints if the fixed network is connected.

\subsection{Future Work}

The future research will continue in the following directions.

1. For the quantized consensus problem, we studied two special kinds of critical dynamics which only admits a single mode. It would be more important and interesting to study the case when the system dynamics consists of more than one mode. Besides, we only considered the case of a fixed and connected network, and discussed about the infeasibility of perturbation techniques in a general directed network due to the order increase of control input, as shown by the examples in Remarks 4.14 and 5.11 . A further study has to be carried out to address the disparity between directed and undirected networks. It is also important to explore the data rate problem under a time-varying network, as in the case of link failures.

2. We note that the initial quantization scale is dependent on a priori bound of initial states, to ensure that the quantizer would not be saturated. However, such a requirement may need 
much communication in a large scale network, and a large quantization scale also leads to slow convergence. Therefore, it makes more sense to design an adaptive encoder/decoder and the corresponding control protocol which is independent of initial states, as is the case in 62. Moreover, noticing that the system output is always corrupted with noise in practice, we shall study the effect of noise on the data rate by a probabilistic analysis.

3. For the optimal consensus problem under set constraint, the current work focuses on single integrators and general convex cost functions. Several further research issues are worthy of exploration. On the one hand, it is meaningful to study the case of double integrators which are widely employed in modeling. On the other hand, a faster convergence is expected for smooth and strongly convex costs. Furthermore, the projection onto the set constraint is only effective for regular sets as noted by Remark 6.3. Therefore, it is more practical to consider inequality and equality constraints.

4. For the optimal consensus problem under kinematic constraints, we assume an exact knowledge of nonlinearity in the EL dynamics which is rarely the case in practice. It is necessary to consider the case of unknown system parameters. In addition, the current analysis yields conservative control inputs and needs an initial estimate of the optimal solution for the design of control gains, which greatly restricts its application. A new control protocol based on input saturation should be investigated. 



\section{Author's Selected Publications}

\section{Journal papers}

1. Z. Qiu, S. Liu, and L. Xie, "Distributed constrained optimal consensus of multi-agent systems," Automatica, vol. 68, pp. 209-215, Jun. 2016.

2. Z. Qiu, L. Xie, and Y. Hong, "Quantized Leaderless and Leader-Following Consensus of HighOrder Multi-Agent Systems With Limited Data Rate," IEEE Trans. on Autom. Control, vol. 61, no. 9, pp. 2432-2447, Sep. 2016. (Regular Paper)

3. Z. Qiu, L. Xie, and Y. Hong, Z. Qiu, L. Xie, and Y. Hong, "Data Rate for Distributed Consensus of Multi-agent Systems with High-order Oscillator Dynamics," IEEE Trans. on Autom. Control, vol. PP, no. 99, pp. 11, 2017.

4. S. Liu, Z. Qiv* and L. Xie, "Convergence Rate Analysis of Distributed Projected Subgradientbased Optimization," to appear on Automatica, 2017.

5. S. Liu, Z. Qiu*, and L. Xie, "Necessary and Sufcient Conditions for Distributed Constrained Optimal Consensus," Submitted to Int. J. of Robust and Nonlinear Control, 2017.

* Co-first author. 


\section{Conference papers}

1. Z. Qiu, Y. Hong, and L. Xie, "Quantized leaderless and leader-following consensus of high-order multi-agent systems with limited data rate," in 52nd IEEE Conference on Decision and Control, Florence, Dec. 2013.

2. S. Liu, Z. Qiu, and L. Xie, "Continuous-time distributed convex optimization with set constraints," in 19th IFAC World Congress, Cape Town, June 2014.

3. Z. Qiu, L. Xie, and Y. Hong, "Data rate for quantized consensus of high-order multi-agent systems with poles on the unit circle," in 53rd IEEE Conference on Decision and Control, Los Angles, Dec. 2014.

4. Y. Long, Z. Qiu, S. Liu, and L. Xie, "Constrained consensus with multiplicative noises," in 12th IEEE International Conference on Control and Automation (ICCA), Kathmandu, June 2016.

5. Z. Qiu, S. Liu, and L. Xie, "Distributed Constrained Consensus with Distance-dependent Measurement Noises," in 6th IFAC Workshop on Distributed Estimation and Control in Networked Systems (NecSys), Tokyo, Sept. 2016.

6. Z. Qiu, Y. Hong, and L. Xie, "Optimal Consensus of Euler-Lagrangian Systems with Kinematic Constraints," in 6th IFAC Workshop on Distributed Estimation and Control in Networked Systems (NecSys), Tokyo, Sept. 2016.

7. Z. Qiu, S. Liu, and L. Xie, "Distributed Constrained Optimal Consensus under Fixed Time Delays", in 14 th International Conference on Control, Automation, Robotics and Vision, Phuket, Nov. 2016.

8. Z. Qiu, H. Zou, L. Xie and Y. Hong, "Consensus-based Parallel Extreme Learning Machine for Indoor Localization", in 59th annual IEEE Global Communications Conference (GLOBECOM), Washington DC, Dec. 2016. 


\section{Bibliography}

[1] A. Jadbabaie, Jie Lin, and A. Morse, "Coordination of groups of mobile autonomous agents using nearest neighbor rules," IEEE Trans. Autom. Control, vol. 48, no. 6, pp. 988-1001, June 2003.

[2] J. Lin, A. S. Morse, and B. D. O. Anderson, "The Multi-Agent Rendezvous Problem. Part 1: The Synchronous Case," SIAM J. Control Optim., vol. 46, no. 6, pp. 2096-2119, Jan. 2007.

[3] —_, "The Multi-Agent Rendezvous Problem. Part 2: The Asynchronous Case," SIAM J. Control Optim., vol. 46, no. 6, pp. 2120-2147, Jan. 2007.

[4] R. Olfati-Saber, "Flocking for Multi-Agent Dynamic Systems: Algorithms and Theory," IEEE Trans. Autom. Control, vol. 51, no. 3, pp. 401-420, Mar. 2006.

[5] D. A. Paley, N. E. Leonard, and R. Sepulchre, "Oscillator models and collective motion: Splay state stabilization of self-propelled particles," in Proceedings of the 44th IEEE Conference on Decision and Control. IEEE, 2005, pp. 3935-3940.

[6] F. Garin and L. Schenato, "A Survey on Distributed Estimation and Control Applications Using Linear Consensus Algorithms," in Networked Control Systems, A. Bemporad, M. Heemels, and M. Johansson, Eds. London: Springer London, 2010, pp. 75-107.

[7] K. You and L. Xie, "Network Topology and Communication Data Rate for Consensusability of Discrete-Time Multi-Agent Systems," IEEE Trans. Autom. Control, vol. 56, no. 10, pp. 22622275, Oct. 2011.

[8] T. Li, M. Fu, L. Xie, and J.-F. Zhang, "Distributed Consensus With Limited Communication Data Rate," IEEE Trans. Autom. Control, vol. 56, no. 2, pp. 279-292, Feb. 2011.

[9] T. Li and L. Xie, "Distributed Coordination of Multi-Agent Systems With Quantized-Observer Based Encoding-Decoding," IEEE Trans. Autom. Control, vol. 57, no. 12, pp. 3023-3037, Dec. 2012 . 
[10] J. Tsitsiklis, "Problems in decentralized decision making and computation," Ph.D. dissertation, MASSACHUSETTS INST OF TECH CAMBRIDGE LAB FOR INFORMATION AND DECISION SYSTEMS, 1984.

[11] K.-K. Oh, M.-C. Park, and H.-S. Ahn, "A survey of multi-agent formation control," Automatica, vol. 53, pp. 424-440, Mar. 2015.

[12] S. Martínez, "Practical multiagent rendezvous through modified circumcenter algorithms," Automatica, vol. 45, no. 9, pp. 2010-2017, Sept. 2009.

[13] X. Wang, Y. Hong, J. Huang, and Z. P. Jiang, "A Distributed Control Approach to A Robust Output Regulation Problem for Multi-Agent Linear Systems," IEEE Trans. Autom. Control, vol. 55, no. 12, pp. 2891-2895, Dec. 2010.

[14] R. Olfati-Saber, "Distributed Kalman filtering for sensor networks," in 2007 46th IEEE Conference on Decision and Control, Dec. 2007, pp. 5492-5498.

[15] A. Nedić and A. Ozdaglar, "Subgradient Methods for Saddle-Point Problems," J. Optim. Theory Appl., vol. 142, no. 1, pp. 205-228, July 2009.

[16] T. Vicsek, A. Czirók, E. Ben-Jacob, I. Cohen, and O. Shochet, "Novel Type of Phase Transition in a System of Self-Driven Particles," Phys. Rev. Lett., vol. 75, no. 6, pp. 1226-1229, Aug. 1995.

[17] R. Olfati-Saber and R. Murray, "Consensus Problems in Networks of Agents With Switching Topology and Time-Delays," IEEE Trans. Autom. Control, vol. 49, no. 9, pp. 1520-1533, Sept. 2004 .

[18] L. Moreau, "Stability of continuous-time distributed consensus algorithms," in Decision and Control, 2004. CDC. 43rd IEEE Conference On, vol. 4. IEEE, 2004, pp. 3998-4003.

[19] — - "Stability of multiagent systems with time-dependent communication links," IEEE Trans. Autom. Control, vol. 50, no. 2, pp. 169-182, Feb. 2005.

[20] W. Ren, "Second-order consensus algorithm with extensions to switching topologies and reference models," in American Control Conference, 200\%. ACC'0\%. IEEE, 2007, pp. 1431-1436.

[21] G. Xie and L. Wang, "Consensus control for a class of networks of dynamic agents," Int. J. Robust Nonlinear Control, vol. 17, no. 10-11, pp. 941-959, July 2007.

[22] W. Yu, G. Chen, and M. Cao, "Some necessary and sufficient conditions for second-order consensus in multi-agent dynamical systems," Automatica, vol. 46, no. 6, pp. 1089-1095, June 2010. 
[23] D. Xie and S. Wang, "Consensus of second-order discrete-time multi-agent systems with fixed topology," J. Math. Anal. Appl., vol. 387, no. 1, pp. 8-16, Mar. 2012.

[24] W. Ren, "Synchronization of coupled harmonic oscillators with local interaction," Automatica, vol. 44, no. 12, pp. 3195-3200, Dec. 2008.

[25] H. Su, X. Wang, and Z. Lin, "Synchronization of coupled harmonic oscillators in a dynamic proximity network," Automatica, vol. 45, no. 10, pp. 2286-2291, Oct. 2009.

[26] L. Ballard, Y. Cao, and W. Ren, "Distributed discrete-time coupled harmonic oscillators with application to synchronised motion coordination," IET Control Theory Appl., vol. 4, no. 5, pp. 806-816, 2010.

[27] P. Wieland, J.-S. Kim, H. Scheu, and F. Allgöwer, "On consensus in multi-agent systems with linear high-order agents," in Proceedings of the IFAC World Congress, Seoul, Korea, vol. 17, 2008, pp. 1541-1546.

[28] F. Jiang, L. Wang, and Y. Jia, "Consensus in leaderless networks of high-order-integrator agents," in American Control Conference, 2009. ACC'09. IEEE, 2009, pp. 4458-4463.

[29] J. H. Seo, H. Shim, and J. Back, "Consensus of high-order linear systems using dynamic output feedback compensator: Low gain approach," Automatica, vol. 45, no. 11, pp. 2659-2664, Nov. 2009.

[30] S. E. Tuna, "Conditions for Synchronizability in Arrays of Coupled Linear Systems," IEEE Transactions on Automatic Control, vol. 54, no. 10, pp. 2416-2420, Oct. 2009.

[31] C. Q. Ma and J. F. Zhang, "Necessary and Sufficient Conditions for Consensusability of Linear Multi-Agent Systems," IEEE Transactions on Automatic Control, vol. 55, no. 5, pp. 1263-1268, May 2010.

[32] N. Chopra and M. W. Spong, "On Exponential Synchronization of Kuramoto Oscillators," IEEE Trans. Autom. Control, vol. 54, no. 2, pp. 353-357, Feb. 2009.

[33] D. V. Dimarogonas and K. J. Kyriakopoulos, "On the Rendezvous Problem for Multiple Nonholonomic Agents," IEEE Trans. Autom. Control, vol. 52, no. 5, pp. 916-922, May 2007.

[34] W. Ren, "Distributed leaderless consensus algorithms for networked Euler-Lagrange systems," Int. J. Control, vol. 82, no. 11, pp. 2137-2149, Nov. 2009.

[35] J. Cortés, "Distributed algorithms for reaching consensus on general functions," Automatica, vol. 44, no. 3, pp. 726-737, Mar. 2008. 
[36] M. Zhu and S. Martínez, "Discrete-time dynamic average consensus," Automatica, vol. 46, no. 2, pp. 322-329, Feb. 2010.

[37] R. Olfati-Saber, J. A. Fax, and R. M. Murray, "Consensus and Cooperation in Networked Multi-Agent Systems," Proc. IEEE, vol. 95, no. 1, pp. 215-233, Jan. 2007.

[38] Y. Cao, W. Yu, W. Ren, and G. Chen, "An Overview of Recent Progress in the Study of Distributed Multi-Agent Coordination," IEEE Trans. Ind. Inform., vol. 9, no. 1, pp. 427-438, Feb. 2013.

[39] S. Knorn, Z. Chen, and R. Middleton, "Overview: Collective Control of Multi-agent Systems," IEEE Trans. Control Netw. Syst., vol. PP, no. 99, pp. 1-1, 2015.

[40] F. Bullo, J. Cortés, and S. Martínez, Distributed Control of Robotic Networks a Mathematical Approach to Motion Coordination Algorithms. Princeton: Princeton University Press, 2009.

[41] W. Ren and Y. Cao, Distributed Coordination of Multi-Agent Networks: Emergent Problems, Models, and Issues. Springer Science \& Business Media, Nov. 2010.

[42] F. Xiao and L. Wang, "Consensus protocols for discrete-time multi-agent systems with timevarying delays," Automatica, vol. 44, no. 10, pp. 2577-2582, Oct. 2008.

[43] R. Carli, F. Fagnani, A. Speranzon, and S. Zampieri, "Communication constraints in the average consensus problem," Automatica, vol. 44, no. 3, pp. 671-684, Mar. 2008.

[44] H. Su, M. Z. Q. Chen, J. Lam, and Z. Lin, "Semi-Global Leader-Following Consensus of Linear Multi-Agent Systems With Input Saturation via Low Gain Feedback," IEEE Trans. Circuits Syst. Regul. Pap., vol. 60, no. 7, pp. 1881-1889, July 2013.

[45] A. Kashyap, T. Başar, and R. Srikant, "Quantized consensus," Automatica, vol. 43, no. 7, pp. 1192-1203, July 2007.

[46] K. Cai and H. Ishii, "Average consensus on general digraphs," in Decision and Control and European Control Conference (CDC-ECC), 2011 50th IEEE Conference On. IEEE, 2011, pp. 1956-1961.

[47] — - "Convergence time analysis of quantized gossip consensus on digraphs," Automatica, vol. 48, no. 9, pp. 2344-2351, Sept. 2012.

[48] M. Zhu and S. Martinez, "On the Convergence Time of Asynchronous Distributed Quantized Averaging Algorithms," IEEE Trans. Autom. Control, vol. 56, no. 2, pp. 386-390, Feb. 2011.

[49] S. Shang, P. Cuff, P. Hui, and S. Kulkarni, "An Upper Bound on the Convergence Time for Quantized Consensus of Arbitrary Static Graphs," IEEE Trans. Autom. Control, vol. 60, no. 4, pp. 1127-1132, Apr. 2015. 
[50] S. R. Etesami and T. Başar, "Convergence Time for Unbiased Quantized Consensus Over Static and Dynamic Networks," IEEE Trans. Autom. Control, vol. 61, no. 2, pp. 443-455, Feb. 2016.

[51] T. Aysal, M. Coates, and M. Rabbat, "Distributed Average Consensus with Dithered Quantization," IEEE Trans. Signal Process., vol. 56, no. 10, pp. 4905-4918, Oct. 2008.

[52] S. Kar and J. M. F. Moura, "Distributed Consensus Algorithms in Sensor Networks: Quantized Data and Random Link Failures," IEEE Trans. Signal Process., vol. 58, no. 3, pp. 1383-1400, Mar. 2010

[53] R. Carli, F. Fagnani, P. Frasca, and S. Zampieri, "Gossip consensus algorithms via quantized communication," Automatica, vol. 46, no. 1, pp. 70-80, Jan. 2010.

[54] J. Lavaei and R. M. Murray, "Quantized Consensus by Means of Gossip Algorithm," IEEE Trans. Autom. Control, vol. 57, no. 1, pp. 19-32, Jan. 2012.

[55] D. Yuan, S. Xu, H. Zhao, and Y. Chu, "Distributed average consensus via gossip algorithm with real-valued and quantized data for $0<\mathrm{q}<1$," Systems $\&$ Control Letters, vol. 59, no. 9, pp. 536-542, Sept. 2010.

[56] J. Fang and H. Li, "Distributed Consensus With Quantized Data via Sequence Averaging," IEEE Trans. Signal Process., vol. 58, no. 2, pp. 944-948, Feb. 2010.

[57] R. Carli, F. Bullo, and S. Zampieri, "Quantized average consensus via dynamic coding/decoding schemes," Int. J. Robust Nonlinear Control, vol. 20, no. 2, pp. 156-175, Jan. 2010.

[58] G. Baldan and S. Zampieri, "An efficient quantization algorithm for solving average-consensus problems," in 2009 European Control Conference (ECC), Aug. 2009, pp. 761-766.

[59] D. Thanou, E. Kokiopoulou, Y. Pu, and P. Frossard, "Distributed Average Consensus With Quantization Refinement," IEEE Trans. Signal Process., vol. 61, no. 1, pp. 194-205, Jan. 2013.

[60] Q. Zhang and J.-F. Zhang, "Quantized Data-Based Distributed Consensus under Directed TimeVarying Communication Topology," SIAM J. Control Optim., vol. 51, no. 1, pp. 332-352, Jan. 2013.

[61] D. Li, Q. Liu, X. Wang, and Z. Yin, "Quantized consensus over directed networks with switching topologies," Syst. Control Lett., vol. 65, pp. 13-22, Mar. 2014.

[62] A. Olshevsky, "Consensus with Ternary Messages," SIAM J. Control Optim., vol. 52, no. 2, pp. 987-1009, Jan. 2014.

[63] Z. Qiu, L. Xie, and Y. Hong, "Quantized Leaderless and Leader-Following Consensus of HighOrder Multi-Agent Systems With Limited Data Rate," IEEE Trans. Autom. Control, vol. 61, no. 9, pp. 2432-2447, Sept. 2016. 
[64] —_ "Data Rate for Distributed Consensus of Multi-agent Systems with High-order Oscillator Dynamics," IEEE Trans. Autom. Control, vol. PP, no. 99, pp. 1-1, 2017.

[65] J. Tsitsiklis, D. Bertsekas, and M. Athans, "Distributed asynchronous deterministic and stochastic gradient optimization algorithms," IEEE Transactions on Automatic Control, vol. 31, no. 9, pp. 803-812, Sept. 1986.

[66] V. Cevher, S. Becker, and M. Schmidt, "Convex Optimization for Big Data: Scalable, randomized, and parallel algorithms for big data analytics," IEEE Signal Process. Mag., vol. 31, no. 5, pp. 32-43, Sept. 2014.

[67] A. H. Sayed, "Adaptation, Learning, and Optimization over Networks," $M A L$, vol. 7, no. 4-5, pp. 311-801, July 2014.

[68] Y. Zhang and Y. Hong, "Distributed optimization design for high-order multi-agent systems," in Control Conference (CCC), 2015 34th Chinese. IEEE, 2015, pp. 7251-7256.

[69] A. Nedić, A. Ozdaglar, and P. Parrilo, "Constrained Consensus and Optimization in Multi-Agent Networks," IEEE Trans. Autom. Control, vol. 55, no. 4, pp. 922-938, Apr. 2010.

[70] P. Lin, W. Ren, and Y. Song, "Distributed multi-agent optimization subject to nonidentical constraints and communication delays," Automatica, vol. 65, pp. 120-131, Mar. 2016.

[71] A. Nedić and A. Olshevsky, "Distributed optimization over time-varying directed graphs," IEEE Trans. Autom. Control, vol. 60, no. 3, pp. 601-615, 2015.

[72] J. C. Duchi, A. Agarwal, and M. J. Wainwright, "Dual Averaging for Distributed Optimization: Convergence Analysis and Network Scaling," IEEE Trans. Autom. Control, vol. 57, no. 3, pp. 592-606, Mar. 2012.

[73] K. I. Tsianos, S. Lawlor, and M. G. Rabbat, "Push-Sum Distributed Dual Averaging for convex optimization," in 2012 IEEE 51st IEEE Conference on Decision and Control (CDC), Dec. 2012, pp. $5453-5458$.

[74] D. Jakovetic, J. Xavier, and J. M. F. Moura, "Fast Distributed Gradient Methods," IEEE Trans. Autom. Control, vol. 59, no. 5, pp. 1131-1146, May 2014.

[75] A. I. Chen and A. Ozdaglar, "A fast distributed proximal-gradient method," in 2012 50th Annual Allerton Conference on Communication, Control, and Computing (Allerton), Oct. 2012, pp. 601-608.

[76] D. Yuan, S. Xu, and H. Zhao, "Distributed primal-dual subgradient method for multiagent optimization via consensus algorithms," Systems, Man, and Cybernetics, Part B: Cybernetics, IEEE Transactions on, vol. 41, no. 6, pp. 1715-1724, 2011. 
[77] M. Zhu and S. Martinez, "On Distributed Convex Optimization Under Inequality and Equality Constraints," IEEE Trans. Autom. Control, vol. 57, no. 1, pp. 151-164, Jan. 2012.

[78] D. Varagnolo, F. Zanella, A. Cenedese, G. Pillonetto, and L. Schenato, "Newton-Raphson Consensus for Distributed Convex Optimization," IEEE Trans. Autom. Control, vol. 61, no. 4, pp. 994-1009, Apr. 2016.

[79] S. Boyd, Distributed Optimization and Statistical Learning via the Alternating Direction Method of Multipliers, ser. Foundations and Trends® in Machine Learning, 2010, vol. 3.

[80] W. Shi, Q. Ling, K. Yuan, G. Wu, and W. Yin, "On the Linear Convergence of the ADMM in Decentralized Consensus Optimization," IEEE Trans. Signal Process., vol. 62, no. 7, pp. 1750-1761, Apr. 2014.

[81] F. Iutzeler, P. Bianchi, P. Ciblat, and W. Hachem, "Explicit Convergence Rate of a Distributed Alternating Direction Method of Multipliers," IEEE Trans. Autom. Control, vol. 61, no. 4, pp. 892-904, Apr. 2016.

[82] Q. Ling, W. Shi, G. Wu, and A. Ribeiro, "DLM: Decentralized Linearized Alternating Direction Method of Multipliers," IEEE Trans. Signal Process., vol. 63, no. 15, pp. 4051-4064, Aug. 2015.

[83] A. Mokhtari, W. Shi, Q. Ling, and A. Ribeiro, "DQM: Decentralized Quadratically Approximated Alternating Direction Method of Multipliers," IEEE Trans. Signal Process., vol. 64, no. 19 , pp. 5158-5173, Oct. 2016.

[84] D. Jakovetić, J. M. F. Moura, and J. Xavier, "Linear Convergence Rate of a Class of Distributed Augmented Lagrangian Algorithms," IEEE Trans. Autom. Control, vol. 60, no. 4, pp. 922-936, Apr. 2015.

[85] J. Xu, S. Zhu, Y. C. Soh, and L. Xie, "Augmented distributed gradient methods for multiagent optimization under uncoordinated constant stepsizes," in 2015 54th IEEE Conference on Decision and Control (CDC). IEEE, Dec. 2015, pp. 2055-2060.

[86] G. Qu and N. Li, "Harnessing Smoothness to Accelerate Distributed Optimization," arXiv:1605.07112 [math], May 2016.

[87] A. Nedić, A. Olshevsky, and W. Shi, "Achieving Geometric Convergence for Distributed Optimization over Time-Varying Graphs," arXiv:160\%.03218 [math], July 2016.

[88] A. Nedić, A. Olshevsky, W. Shi, and C. A. Uribe, "Geometrically Convergent Distributed Optimization with Uncoordinated Step-Sizes," arXiv:1609.05877 [cs, math, stat], Sept. 2016. 
[89] J. Wang and N. Elia, "Control approach to distributed optimization," in Communication, Control, and Computing (Allerton), 2010 48th Annual Allerton Conference On. IEEE, 2010, pp. $557-561$.

[90] B. Gharesifard and J. Cortes, "Distributed Continuous-Time Convex Optimization on WeightBalanced Digraphs," IEEE Trans. Autom. Control, vol. 59, no. 3, pp. 781-786, Mar. 2014.

[91] S. S. Kia, "A distributed dynamical solver for an optimal resource allocation problem over networked systems," in 2015 54th IEEE Conference on Decision and Control (CDC), Dec. 2015, pp. $7482-7487$.

[92] Z. Qiu, S. Liu, and L. Xie, "Distributed constrained optimal consensus of multi-agent systems," Automatica, vol. 68, pp. 209-215, June 2016.

[93] Q. Liu and J. Wang, "A Second-Order Multi-Agent Network for Bound-Constrained Distributed Optimization," IEEE Trans. Autom. Control, vol. 60, no. 12, pp. 3310-3315, Dec. 2015.

[94] X. Zeng, P. Yi, and Y. Hong, "Distributed Continuous-Time Algorithm for Constrained Convex Optimizations via Nonsmooth Analysis Approach," arXiv:1510.07386 [math], Oct. 2015.

[95] S. Yang, Q. Liu, and J. Wang, "A Multi-Agent System with a Proportional-Integral Protocol for Distributed Constrained Optimization," IEEE Trans. Autom. Control, vol. PP, no. 99, pp. $1-1,2016$

[96] D. Mateos-Núñez and J. Cortés, "Distributed saddle-point subgradient algorithms with Laplacian averaging," arXiv:1510.05169 [math], Oct. 2015.

[97] P. Yi, Y. Hong, and F. Liu, "Initialization-free Distributed Algorithms for Optimal Resource Allocation with Feasibility Constraints and its Application to Economic Dispatch of Power Systems," arXiv:1510.08579 [math], Oct. 2015.

[98] Q. Zhang, B.-C. Wang, and J.-F. Zhang, "Distributed dynamic consensus under quantized communication data," Int. J. Robust Nonlinear Control, vol. 25, no. 11, pp. 1704-1720, July 2015.

[99] Z. Deng and Y. Hong, "Multi-Agent Optimization Design for Autonomous Lagrangian Systems," Un. Sys., vol. 04, no. 01, pp. 5-13, Jan. 2016.

[100] R. A. Horn and C. R. Johnson, Matrix Analysis. Cambridge university press, 2012.

[101] W. Ren, "On Consensus Algorithms for Double-Integrator Dynamics," IEEE Trans. Autom. Control, vol. 53, no. 6, pp. 1503-1509, July 2008. 
[102] F. Xiao and L. Wang, "State consensus for multi-agent systems with switching topologies and time-varying delays," Int. J. Control, vol. 79, no. 10, pp. 1277-1284, Oct. 2006.

[103] J.-B. Hiriart-Urruty and C. Lemaréchal, Fundamentals of Convex Analysis. Berlin, Heidelberg: Springer Berlin Heidelberg, 2001.

[104] G. Shi and Y. Hong, "Global target aggregation and state agreement of nonlinear multi-agent systems with switching topologies," Automatica, vol. 45, no. 5, pp. 1165-1175, May 2009.

[105] K. You, N. Xiao, and L. Xie, Analysis and Design of Networked Control Systems. Springer, Jan. 2015.

[106] D. Mellinger and V. Kumar, "Minimum snap trajectory generation and control for quadrotors," in Robotics and Automation (ICRA), 2011 IEEE International Conference On. IEEE, 2011, pp. 2520-2525.

[107] T. Li and L. Xie, "Distributed consensus over digital networks with limited bandwidth and time-varying topologies," Automatica, vol. 47, no. 9, pp. 2006-2015, Sept. 2011.

[108] A. P. Seyranian and A. A. Mailybaev, Multiparameter Stability Theory with Mechanical Applications. World Scientific, 2003, vol. 13.

[109] H. Baumgärtel, Analytic Perturbation Theory for Matrices and Operators. Springer, 1985, vol. 15 .

[110] M. I. Vishik and L. A. Lyusternik, "The solution of some perturbation problems for matrices and selfadjoint or non-selfadjoint differential equations I," Russ. Math. Surv., vol. 15, no. 3, p. 1, 1960.

[111] J. Moro and F. Dopico, "Low Rank Perturbation of Jordan Structure," SIAM. J. Matrix Anal. E Appl., vol. 25, no. 2, pp. 495-506, Jan. 2003.

[112] Y. Hong, J. Hu, and L. Gao, "Tracking control for multi-agent consensus with an active leader and variable topology," Automatica, vol. 42, no. 7, pp. 1177-1182, July 2006.

[113] C.-T. Chen, Linear System Theory and Design. Oxford University Press, Inc., 1995.

[114] Y. Meng, T. Li, and J. F. Zhang, "Coordination Over Multi-Agent Networks with Unmeasurable States and Finite-Level Quantization," IEEE Trans. Autom. Control, vol. PP, no. 99, pp. 1-1, 2016.

[115] R. Sprugnoli, An Introduction to Mathematical Methods in Combinatorics. CreateSpace Independent Publishing Platform, Oct. 2014. 
[116] — Riordan Array Proofs of Identities in Gould's Book. Published electronically at http://www. dsi. unifi. it/resp/GouldBK. pdf, 2007.

[117] J. Nocedal and S. Wright, Numerical Optimization. Springer Science \& Business Media, Dec. 2006.

[118] I. Lobel and A. Ozdaglar, "Distributed Subgradient Methods for Convex Optimization Over Random Networks," IEEE Trans. Autom. Control, vol. 56, no. 6, pp. 1291-1306, June 2011.

[119] G. Shi, A. Proutiere, and K. H. Johansson, "Distributed Optimization: Convergence Conditions from a Dynamical System Perspective," ArXiv Prepr. ArXiv12106685, 2012.

[120] A. F. Filippov and F. M. Arscott, Differential Equations with Discontinuous Righthand Sides: Control Systems. Springer Science \& Business Media, 1988, vol. 18.

[121] V. Lakshmikantham and S. Leela, Differential and Integral Inequalities. Academic press, 1969.

[122] J. M. Danskin, "The theory of max-min, with applications," SIAM J. Appl. Math., vol. 14, no. 4, pp. 641-664, 1966.

[123] G. Shi, K. H. Johansson, and Y. Hong, "Reaching an Optimal Consensus: Dynamical Systems That Compute Intersections of Convex Sets," IEEE Trans. Autom. Control, vol. 58, no. 3, pp. 610-622, Mar. 2013.

[124] L. Guo, Time-Varying Stochastic Systems: Stability, Estimation and Control. Jilin Science and Technology Press, Changchun, 1993.

[125] A. Nedić and A. Olshevsky, "Stochastic Gradient-Push for Strongly Convex Functions on TimeVarying Directed Graphs," IEEE Trans. Autom. Control, vol. 61, no. 12, pp. 3936 - 3947, 2016.

[126] C. Xi and U. A. Khan, "Distributed Subgradient Projection Algorithm over Directed Graphs," IEEE Trans. Autom. Control, vol. PP, no. 99, pp. 1-1, 2016.

[127] S. Liu, T. Li, and L. Xie, "Distributed Consensus for Multiagent Systems with Communication Delays and Limited Data Rate," SIAM J. Control Optim., vol. 49, no. 6, pp. 2239-2262, Jan. 2011.

[128] E. Seneta, Non-Negative Matrices and Markov Chains. Springer Science \& Business Media, July 2006.

[129] M. W. Spong and M. Vidyasagar, Robot Dynamics And Control. Wiley India Pvt. Limited, Aug. 2008.

[130] Y. Zhang and Y. Hong, "Distributed optimization design for second-order multi-agent systems," in Control Conference (CCC), 2014 33rd Chinese. IEEE, 2014, pp. 1755-1760. 